\title{
Influence of high-intensity interval training in adolescents with asthma
}

Winn, Charles O.N.

How to cite:

Winn, Charles O.N. (2018) Influence of high-intensity interval training in adolescents with asthma. Doctoral thesis, Swansea University.

http://cronfa.swan.ac.uk/Record/cronfa46062

Use policy:

This item is brought to you by Swansea University. Any person downloading material is agreeing to abide by the terms of the repository licence: copies of full text items may be used or reproduced in any format or medium, without prior permission for personal research or study, educational or non-commercial purposes only. The copyright for any work remains with the original author unless otherwise specified. The full-text must not be sold in any format or medium without the formal permission of the copyright holder. Permission for multiple reproductions should be obtained from the original author.

Authors are personally responsible for adhering to copyright and publisher restrictions when uploading content to the repository.

Please link to the metadata record in the Swansea University repository, Cronfa (link given in the citation reference above.)

http://www.swansea.ac.uk/library/researchsupport/ris-support/ 


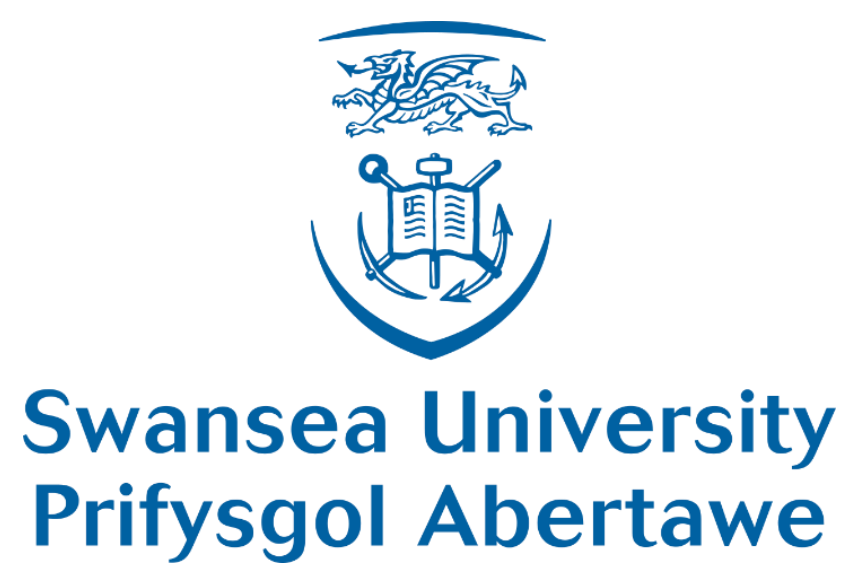

Influence of high-intensity interval training in adolescents

with asthma

The x4a trial: eXercise for Asthma

\section{CHARLES OLIVER NATHAN WINN}

BSc (Hons) MSc

Submitted to Swansea University in fulfilment of the requirements for the Degree of Doctor of Philosophy

Swansea University

2018 


\begin{abstract}
$\underline{\text { Abstract }}$
Low levels of fitness are associated with increased body mass index (BMI), both of which are related to quality of life (QoL) and the occurrence and severity of asthma. Therefore, the purpose of this thesis was to co-develop and implement a six-month high-intensity interval training (HIIT) intervention targeting aerobic fitness, BMI and QoL improvements in adolescents with asthma.

Six-hundred and sixteen adolescents (13.0 1 1.1years; 155 asthma; $85 \%$ mild asthma as defined by medication-step) participated in the study, with 223 enrolled in the school based HIIT intervention (50 asthma), consisting of 10-30second bouts at $>90 \%$ age-predicted maximum heart-rate with equal rest, 3x30minutes/week. Formative group interviews to elicit the views of adolescents with and without asthma on asthma and exercise were used to inform the design of the intervention. Anthropometrics, aerobic fitness, lung function and QoL were measured at baseline, mid-point and post-intervention to evaluate the intervention's impact; follow-up measures were also taken to evaluate sustainability.

Focus groups revealed participants with asthma had a fear of asthma attack through exercise, although fear did not act as a barrier, and that the intervention should be varied to circumvent activity-specific anxieties. Participants with asthma were found to have higher BMI and lower physical activity in comparison to their peers, but no difference in cardiorespiratory fitness. The intervention elicited significant improvements in peak cardiorespiratory fitness, irrespective of asthma, while maintaining BMI which increased in the controls; no changes were observed in the sub-maximal exercise parameters, QoL or lung function.

The present findings suggest that HIIT may be effective at increasing peak cardiorespiratory fitness and preventing increases in BMI in adolescents, irrespective of asthma status. Overall, the series of studies show that asthma does not deleteriously influence maximal and sub-maximal aerobic fitness, or trainability in adolescents, and that HIIT is a safe exercise modality for adolescents with asthma.
\end{abstract}




\section{$\underline{\text { Declarations and Statements }}$}

This work has not previously been accepted in substance for any degree and is not being concurrently submitted in candidature for any degree.

Signed:

(candidate)

Date:

\section{STATEMENT 1}

This thesis is the result of my own investigations, except where otherwise stated.

Other sources are acknowledged by footnotes giving explicit references. A bibliography is appended.

Signed:

(candidate)

Date:

\section{STATEMENT 2}

I hereby give consent for my thesis, if accepted, to be available for photocopying and for inter-library loan, and for the title and summary to be made available to outside organisations.

Signed: (candidate)

Date: 


\section{Contents page}

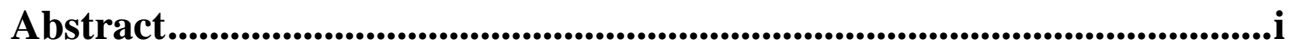

Declarations and Statements...................................................................................ii

Contents page ........................................................................................ iii

Acknowledgements .................................................................................

List of tables..............................................................................................................xii

List of Figures.....................................................................................................xiv

Definitions of abbreviations and symbols ...................................................... $\mathrm{xv}$

Scientific output

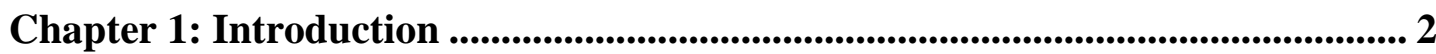

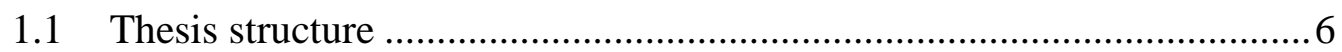

Chapter 2: Literature Review ............................................................................... 10

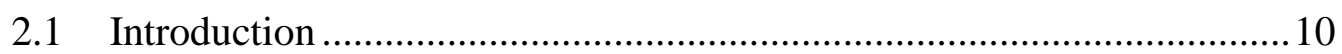

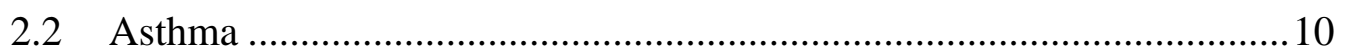

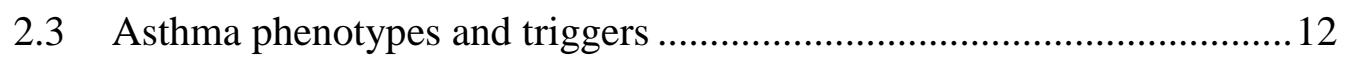

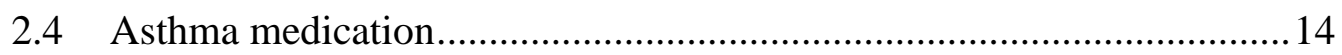

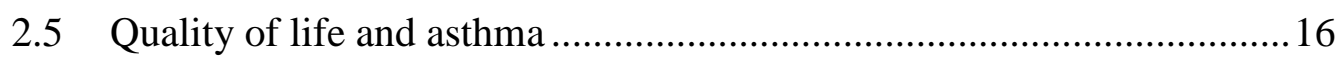

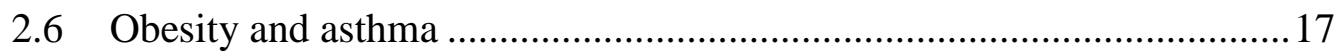

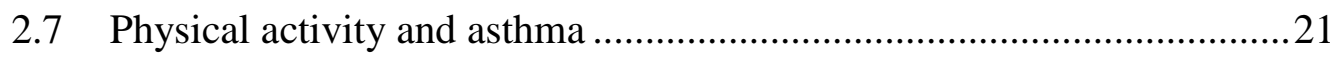

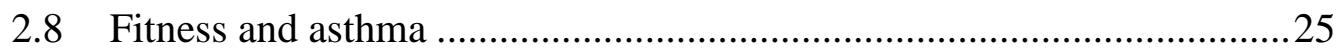

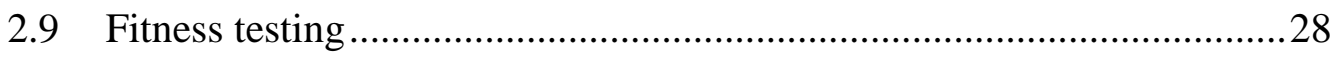


2.9.1 Gas exchange threshold

2.9.2 Gain 31

2.9.3 Mean response time 31

2.9.4 Oxygen uptake kinetics 32

2.9.5 Training effects of $\dot{\mathrm{V}} \mathrm{O}_{2}$ kinetics .36

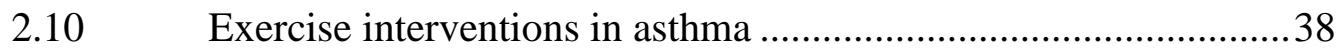

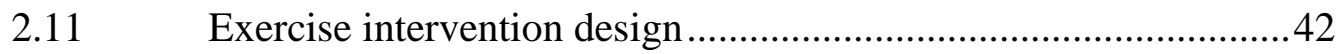

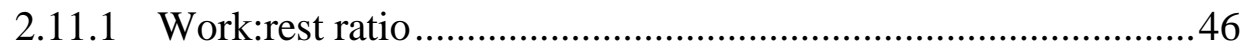

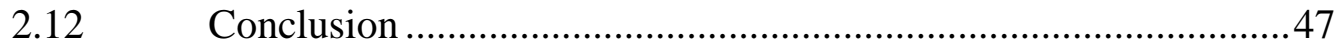

Chapter 3: General Methods.................................................................................5 50

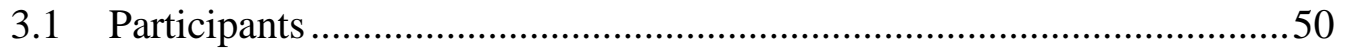

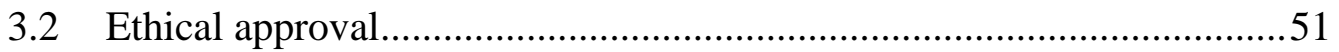

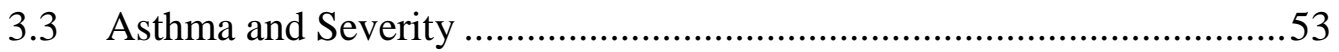

3.3.1 Asthma medication ...................................................................53

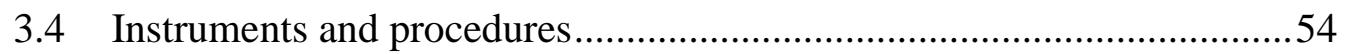

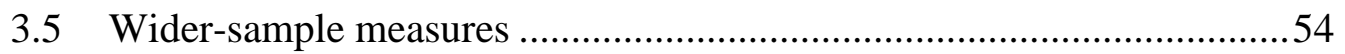

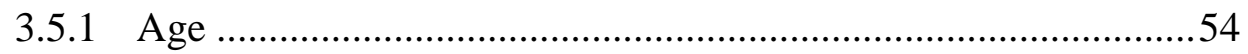

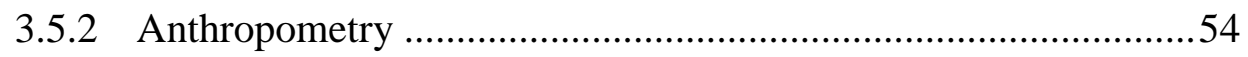

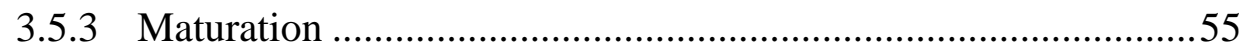

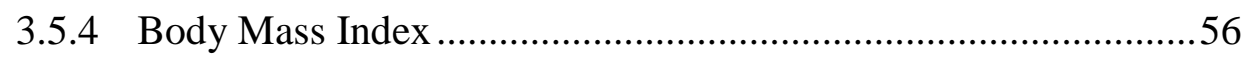

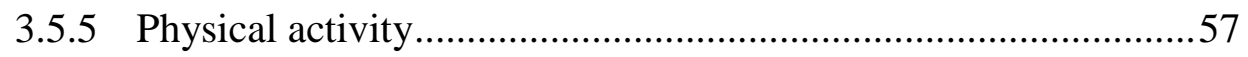


3.5.7 Fractional exhaled Nitric Oxide ............................................58

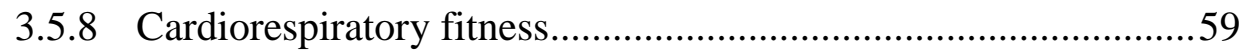

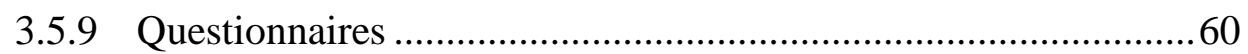

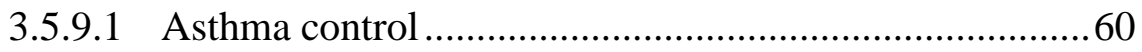

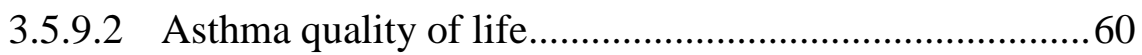

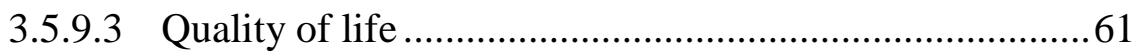

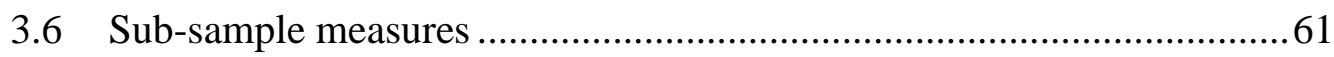

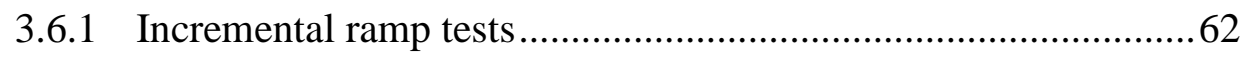

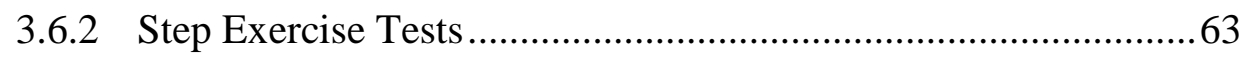

3.6.3 Measurement of gas exchange parameters ..............................63

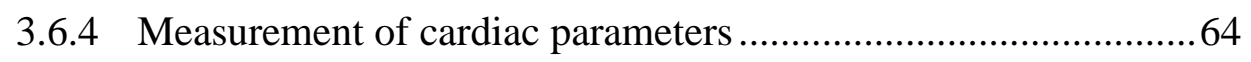

3.6.5 Measurement of muscle oxygenation parameters .....................64

3.6.6 Determination of the gas exchange threshold and $\Delta 40 \%$

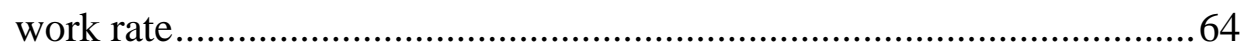

3.6.7 Analysis of $\dot{\mathrm{VO}}_{2}$ response to step exercise tests ......................65

3.6.8 Analysis of the heart rate and $[\mathrm{HHb}]$ response .........................66

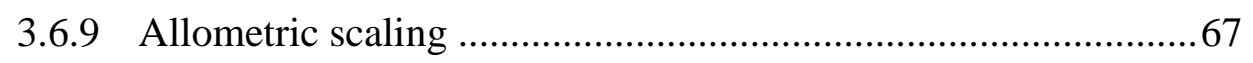

\section{Chapter 4: Perceptions of asthma and exercise in adolescents with and without}

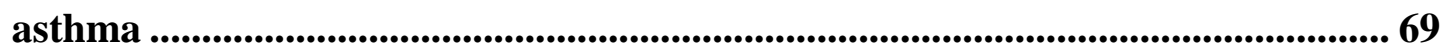

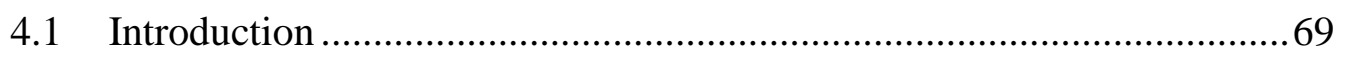

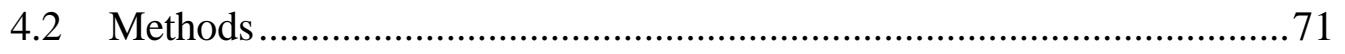


4.2.1 Participants

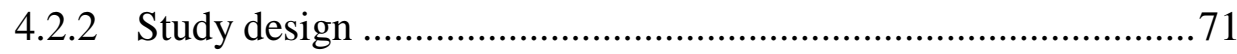

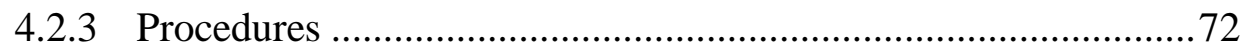

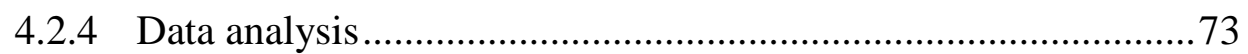

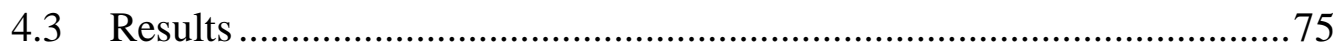

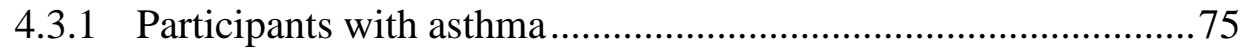

4.3.2 Participants without asthma...................................................... 77

4.3.3 Views on an exercise intervention .......................................... 80

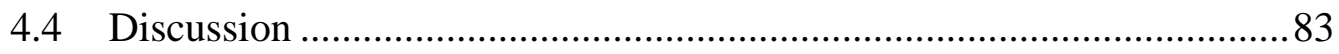

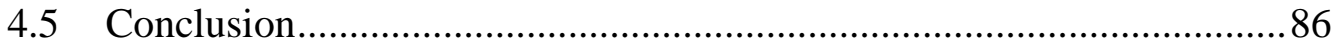

\section{Chapter 5: Investigating the relationship between asthma, body mass and} aerobic fitness in adolescents .......................................................................... 89

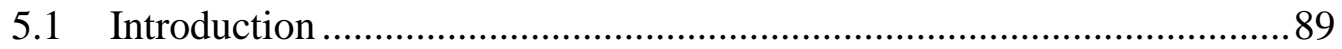

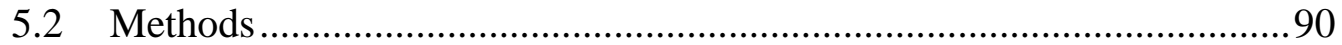

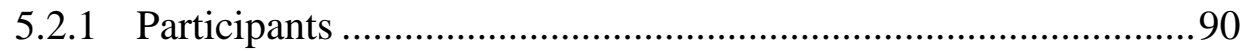

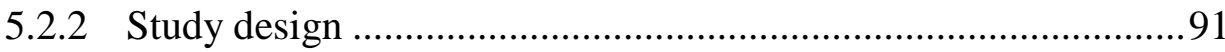

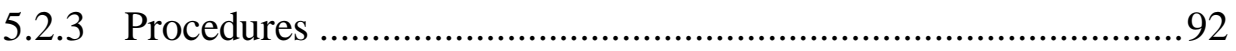

5.2.3.1 Anthropometrics ...................................................... 92

5.2.3.2 Physical activity ....................................................... 92

5.2.3.3 Lung function ........................................................ 92

5.2.3.4 Fractional Exhaled Nitric Oxide ...................................93 
5.2.3.6 Asthma-related quality of life ........................................93

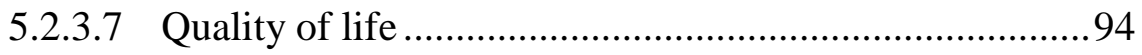

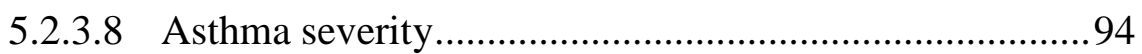

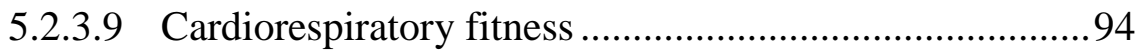

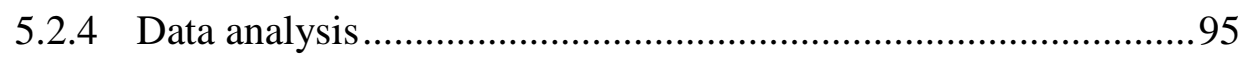

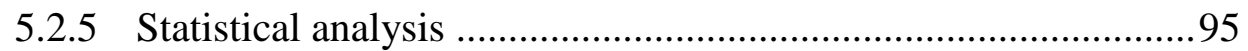

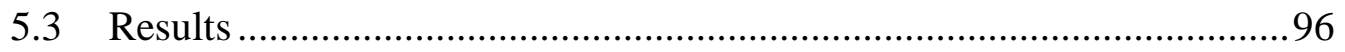

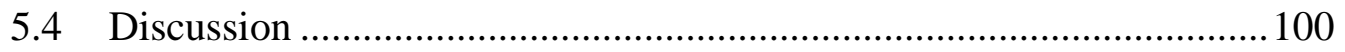

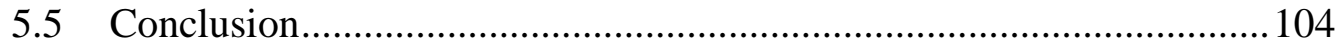

\section{Chapter 6: Effect of high-intensity interval training in adolescents with}

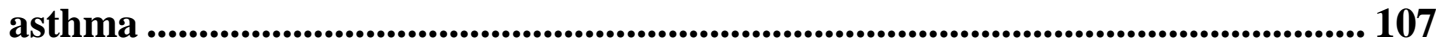

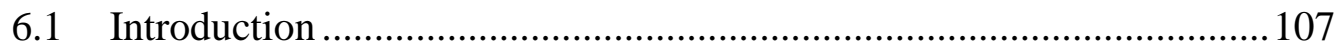

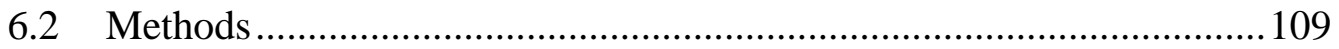

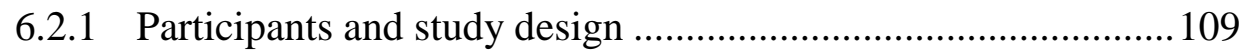

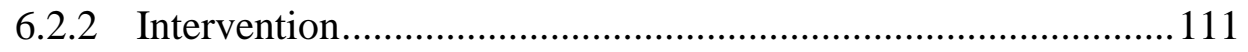

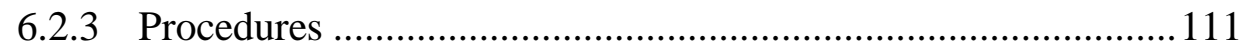

6.2.3.1 Anthropometrics.................................................... 111

6.2.3.2 Lung Function ....................................................... 112

6.2.3.3 Fractional Exhaled Nitric Oxide ................................. 112

6.2.3.4 Asthma control ...................................................... 112 
6.2.3.5 Asthma-related quality of life

6.2.3.6 Quality of life

6.2.3.7 Cardiorespiratory fitness

6.2.4 Data analysis

6.2.5 Statistical analysis 116

6.3 Results 116

6.3.1 Lung function 117

6.3.2 Asthma control and quality of life

6.3.3 Body Mass Index

6.3.4 20-metre shuttle run 120

6.3.5 Incremental ramp test 120

6.3.6 Intervention intensity 122

6.3.7 Correlations 122

6.4 Discussion 122

6.5 Conclusion 126

\section{Chapter 7: Effect of high-intensity interval training on pulmonary oxygen} uptake kinetics in adolescents with asthma

7.1 Introduction 130

7.2 Methods 131

7.2.1 Participants and study design 131

7.2.2 Intervention 132 


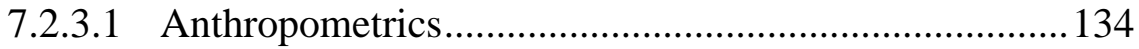

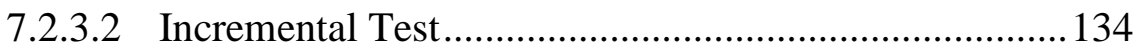

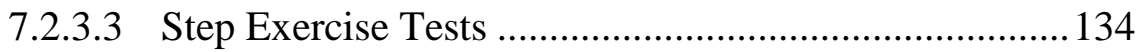

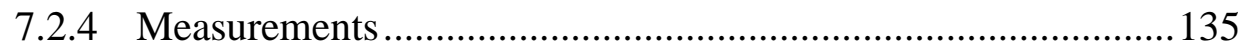

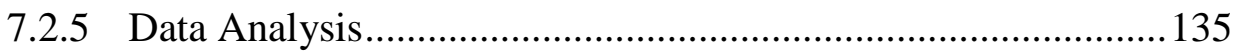

7.2.5.1 Oxygen Uptake Kinetic Analysis ................................. 136

7.2.5.2 $[\mathrm{HHb}] \&$ Heart Rate Kinetics Analysis........................ 137

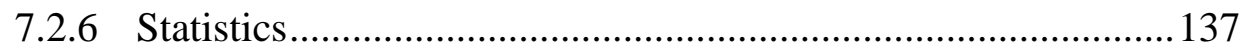

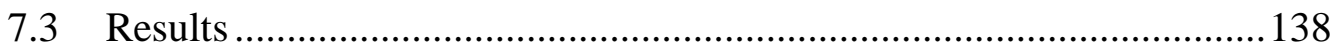

7.3.1 Incremental Ramp Exercise................................................. 138

7.3.2 Constant Work Rate Exercise .................................................. 139

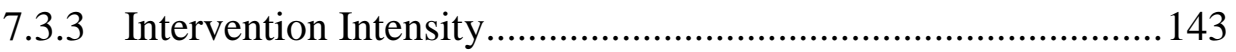

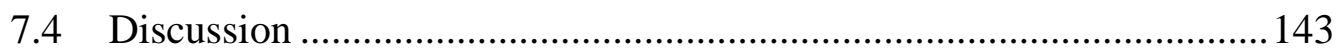

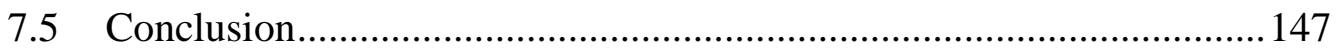

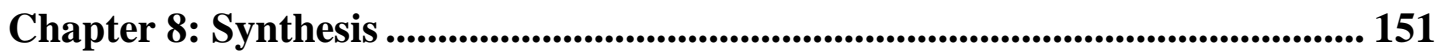

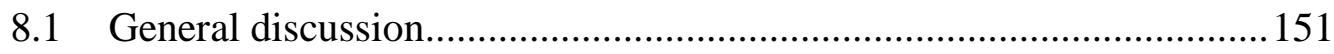

8.1.1 Perception vs. ability of adolescents with asthma ..................... 151

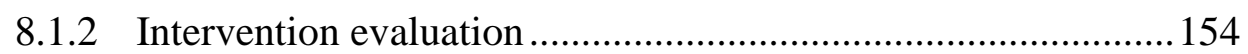

8.1.3 Effect of HIIT on asthma, fitness, obesity and physical

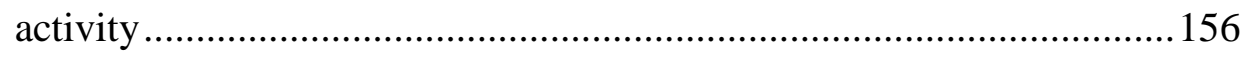


8.2 Study strengths and weaknesses

8.3 Implications for future research .................................................... 161

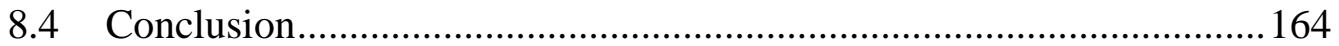

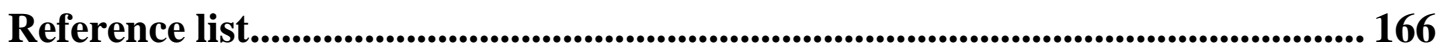

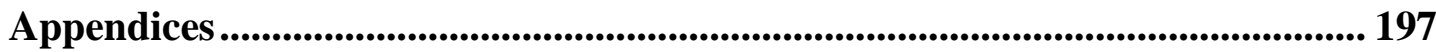

Appendix A: Information sheets and consent forms ...................................... 197

Head teacher information sheets ....................................................... 197

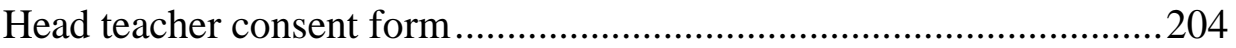

Parental/guardian information sheets ...............................................205

Parental/guardian consent form....................................................212

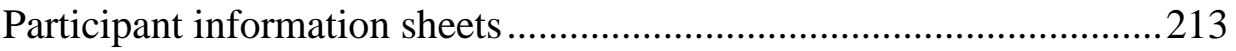

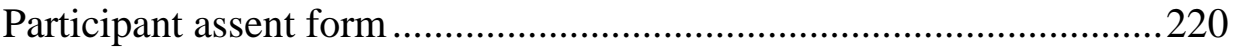

Appendix B: Self-report Tanner stage scale ...............................................222

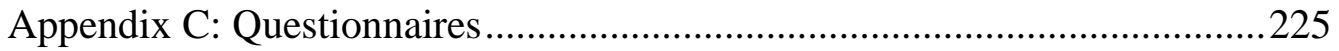

Pediatric Quality of Life Inventory ................................................225

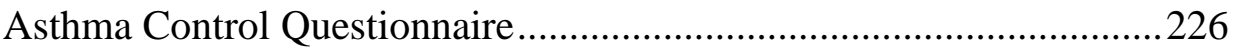

Paediatric Asthma Quality of Life Questionnaire ...............................228

Healthcare Resource Questionnaire ..................................................232

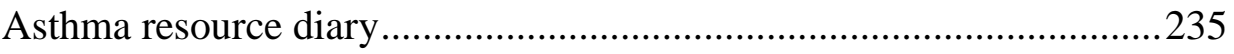




\section{$\underline{\text { Acknowledgements }}$}

I would like to start by giving my utmost thanks to Dr. Melitta McNarry and Prof. Gwyneth Davies for their help and support throughout this research, without their knowledge of the area and their guidance I would not have a thesis to present. I would also like to thank William Eddolls, Nicholas Wade and Sam Crossley for their help and support throughout the $\mathrm{PhD}$ and in Sam's case, most of my life. To all other research students who helped out at some point, the days would have been impossible without you, so thank you! I must also thank Prof. Gareth Stratton, Prof. Andrew Wilson and Dr. Kelly Mackintosh, for their valuable contributions to the project. I would also like to give thanks to the pupils and staff of the schools involved with the planning and execution of the measures.

This work is funded by the Asthma UK Centre for Applied Research [AUK-AC2012-01] and Swansea University Medical School. Commando Joe’s ${ }^{\circledR}$ implemented the intervention and I would like to thank Matthew Hudson for his help running the sessions.

Finally, I would like to thank my family, Gervase, Jane and Jamie for their unwavering support throughout my life, without you I wouldn't have been in a position to undertake this research and more importantly get to the end of it. To my fiancée Sophie, I do not have the words to thank you for putting up with me throughout the last few years... you're stuck with me now! 


\section{List of tables}

\section{Chapter 4}

Table 4.1. Example interview questions .74

\section{Chapter 5}

Table 5.1. Anthropometric measures for participants with and without asthma .......91

Table 5.2. Characteristics of participants with and without asthma .98

Table 5.3. Incremental ramp test results for participants with and without asthma... 99

Table 5.4. Binary logistic regression analysis to test potential association with asthma from Body Mass Index, Moderate-to-Vigorous Physical Activity and 20metre shuttle run. 99

\section{Chapter 6}

Table 6.1. Anthropometric measures for participants within intervention and control for asthma and without asthma

Table 6.2 Detailed examples of exercises

Table 6.3. Example exercise sessions from the high-intensity interval training intervention

Table 6.4. Lung function measures for participants within intervention and control for asthma and without asthma

Table 6.5. 20-metre shuttle run and quality of life questionnaires for participants within intervention and control for asthma and without asthma

Table 6.6. Incremental ramp test results for participants within intervention and control for asthma and without asthma..... 


\section{Chapter 7}

Table 7.1. Anthropometric characteristics according to participant condition

Table 7.2. Pulmonary Oxygen Uptake responses during peak and heavy intensity exercise according to participant condition. 140

Table 7.3. Heart Rate and $[\mathrm{HHb}]$ responses during heavy intensity exercise according to participant condition. 


\section{$\underline{\text { List of Figures }}$}

\section{Chapter 3}

Figure 3.1. Flow chart outlining the school recruitment process, group allocation and intervention completion rate .52

\section{Chapter 4}

Figure 4.1. Adolescents with asthma - pen profile of perceptions of asthma and exercise

Figure 4.2. Adolescents without asthma - pen profile of perceptions of asthma and exercise

Figure 4.3. Pen profile of adolescents' perceptions of an exercise intervention. .82

\section{Chapter 7}

Figure 7.1. Pulmonary oxygen uptake response during heavy-intensity exercise in asthma

Figure 7.2. Pulmonary oxygen uptake response during heavy intensity exercise in non-asthma 


\section{Definitions of abbreviations and symbols}

A1

ACQ

ANCOVA

ANOVA

B

b $\cdot \min ^{-1}$

BM

BMI

BTS

CI

CIET

cm

CWR

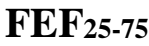

FEF $_{25-75} \%$ predicted

ERS

FeNO

FEV 1

FEV $_{1} \%$ predicted

FVC

FVC \% predicted

G

GET

GINA

HIIE

HIIT

HR
Amplitude of the primary component

Asthma control questionnaire

Analysis of covariance

Analysis of variance

Boy

beats per minute

Body mass

Body mass index

British Thoracic Society

Confidence interval

Constant-intensity exercise training

Centimetre

Constant work rate

Forced Expiratory Flow between $25-75 \%$ of vital capacity

Forced Expiratory Flow between $25-75 \%$ of vital capacity expressed as a percentage of predicted normative values

European Respiratory Society

Fractional exhaled nitric oxide

Forced Expiratory Volume in 1 second

Forced Expiratory Volume in 1 second expressed as a percentage of predicted normative values

Forced Vital Capacity

Forced Vital Capacity expressed as a percentage of predicted normative values

Girl

Gas exchange threshold

Global Initiative for Asthma

High-intensity interval exercise

High-intensity interval training

Heart rate 


\begin{tabular}{|c|c|}
\hline ISAK & International Society for the Advancement of Kinathropometry \\
\hline $\mathbf{l} \cdot \mathbf{m i n}^{-1}$ & Litres per minute \\
\hline $\mathbf{l} \cdot \mathbf{m i n}^{-1} \cdot \mathbf{k g}^{-1}$ & Litres per minute per kilogram \\
\hline $\mathbf{m}$ & Metres \\
\hline MAS & Maximal aerobic speed \\
\hline $\min$ & Minute \\
\hline $\mathbf{m l} \cdot \mathbf{m i n}^{-1} \cdot \mathbf{W}^{-1}$ & Millilitres per minute per Watt \\
\hline MPA & Moderate physical activity \\
\hline MRT & Mean response time \\
\hline MRT $_{1}$ & Mean response time for $\mathrm{S} 1$ \\
\hline MRT $_{\mathbf{T}}$ & Mean response time for $\mathrm{S} 1+\mathrm{S} 2$ \\
\hline MVPA & Moderate-to-vigorous physical activity \\
\hline $\mathbf{n}$ & Sample size \\
\hline NHS & National Health Service \\
\hline NIRS & Near infrared spectroscopy \\
\hline $\mathbf{O}_{2}$ & Oxygen \\
\hline $\mathbf{O R}$ & Odds ratio \\
\hline PAQLQ & Paediatric Asthma Quality of Life Questionnaire \\
\hline PedsQL & Pediatric Quality of Life Inventory \\
\hline PEF & Peak Expiratory Flow \\
\hline $\begin{array}{l}\text { PEF \% } \\
\text { predicted }\end{array}$ & $\begin{array}{l}\text { Peak Expiratory Flow expressed as a percentage of predicted normative } \\
\text { values }\end{array}$ \\
\hline PhH & Physical score \\
\hline PHV & Peak height velocity \\
\hline ppb & Parts per billion \\
\hline PsH & Psychological score \\
\hline QoL & Quality of life \\
\hline $\mathbf{r}$ & Pearson's correlation coefficient \\
\hline rpm & Revolutions per minute \\
\hline $\mathbf{S}$ & Seconds \\
\hline $\mathbf{S}_{1}$ & 1-minute into ramp to GET \\
\hline $\mathbf{S}_{2}$ & GET to peak $\dot{\mathrm{VO}} 2$ \\
\hline
\end{tabular}




\begin{tabular}{|c|c|}
\hline SD & Standard deviation \\
\hline SE & Standard error \\
\hline $\mathbf{S}_{\mathbf{t}}$ & Total range $\mathrm{S} 1+\mathrm{S} 2$ \\
\hline $\mathbf{t}$ & Time \\
\hline TD & Time delay \\
\hline$\dot{V} \mathrm{CO}_{2}$ & Carbon dioxide output \\
\hline$\dot{\boldsymbol{V}}_{\mathbf{E}}$ & Minute ventilation \\
\hline$\dot{V} \mathbf{O}_{2}$ & Oxygen uptake \\
\hline$\dot{V} \mathbf{O}_{2 \max }$ & Maximal oxygen uptake \\
\hline VPA & Vigorous physical activity \\
\hline $\mathbf{W}$ & Watts \\
\hline $\mathbf{W} \cdot \mathbf{m i n}^{-1}$ & Watts per minute \\
\hline WHO & World Health Organisation \\
\hline WR & Work rate \\
\hline B & coefficient \\
\hline$\Delta$ & Delta change \\
\hline$\eta_{p^{2}}^{2}$ & Eta-squared \\
\hline$\tau$ & Time constant \\
\hline$\tau^{\prime}$ & Mean response time \\
\hline$\% \Delta$ & $\%$ difference between GET and peak Vo2 \\
\hline$[\mathbf{H H b}]$ & Deoxygenated haemoglobin and myoglobin \\
\hline
\end{tabular}




\section{$\underline{\text { Scientific output }}$}

\section{Conferences}

Oral presentation at the Asthma UK Centre for Applied Research Annual Scientific Meeting, The x4a trial: eXercise for Asthma, Bristol UK January 2018

Oral presentation at the Asthma UK Centre for Applied Research joint centre event, The effects of a six-month high-intensity interval training intervention on aerobic fitness and fatness in adolescents with asthma, London UK September 2017

Oral presentation at the European College of Sport Science annual congress, The influence of a six-month high-intensity interval training intervention on the pulmonary oxygen uptake kinetics in adolescents with and without asthma, Essen Germany July 2017

Oral presentation at the Pan Wales conference, The influence of a six-month highintensity interval training intervention on the pulmonary oxygen uptake kinetics in adolescents with and without asthma, Swansea UK April 2017

Oral presentation at the Swansea University Medical School Postgraduate conference, Perceptions of asthma and exercise in children and adolescents, Swansea UK December 2016

Oral presentation at the Asthma UK Centre for Applied Research Annual Scientific Meeting, Perceptions of asthma and exercise in children and adolescents, Edinburgh UK November 2016

Oral presentation at the European Respiratory Society International Congress, Effect of high-intensity exercise on aerobic performance and airway inflammation in asthma, London UK September 2016

Poster presentation at Swansea University Medical School Postgraduate conference, The $x 4$ a study: the eXercise for asthma study, Swansea UK December 2015 


\section{Publications}

Eddolls WTB, McNarry MA, Lester L, Winn CON, Stratton G, Mackintosh KA. The association between physical activity, fitness and body mass index on mental well-being and quality of life in adolescents. Qual Life Res. 2018, [online].

Winn CON, Mackintosh KA, Eddolls WTB, Stratton G, Wilson AM, Rance JY, Doull IJM, McNarry MA, Davies GA. Perceptions of asthma and exercise in adolescents with and without asthma. J Asthma. 2017:30,1-9.

Eddolls WTB, McNarry MA, Stratton G, Winn CON, Mackintosh KA. HighIntensity Interval Training Interventions in Children and Adolescents: A Systematic Review. Sports Med. 2017:47, 2363-2374. 


\section{CHAPTER 1}

INTRODUCTION 


\section{Chapter 1: Introduction}

Asthma was first defined by Salter in 1860 who characterised the condition as "Paroxysmal dyspnoea of a peculiar character with intervals of healthy respiration between attacks". More recently, the Global Initiative for Asthma (GINA, Global Initiative for Asthma, 2017) defined asthma as: "a heterogeneous disease, usually characterised by chronic airway inflammation. It is defined by the history of respiratory symptoms such as wheeze, shortness of breath, chest tightness and cough that vary over time and in intensity, together with variable expiratory airflow limitation". However, as asthma is heterogeneous, there is no gold standard definition and the diagnosis is a clinical one. In the UK, asthma affects 1 in 12 adults and, with one of the highest prevalence rates in the world, 1 in 11 children suffer from the condition (Asthma UK, 2017). Asthma is associated with a significant patient burden, affecting people throughout their lifetime and has a significant economic impact. Indeed, the cost of asthma is estimated to be over $£ 1.1$ billion in the UK, with the majority spent on primary care $(74 \%)$, of which $81 \%$ is due to community prescribing (Mukherjee et al., 2016). With no current cure, there is a need to find cost-effective ways to reduce symptoms, improve asthma control and quality of life and reduce the economic burden of asthma.

There are many triggers for asthma such as pets, dust or pollen (Asthma UK, 2018). Triggers vary between individuals who may also have more than one trigger; a major trigger for many is exercise, known as Exercise Induced Bronchoconstriction (EIB) and defined as airway narrowing or obstruction associated with exercise ( $\mathrm{Lu}$ et al., 2016). The prevalence of EIB in children is debated but has been variously reported at between 40-90\% (Wanrooij et al., 2014) and is usually manifest during, or shortly after, exercise. Consequently, some children fear exercise due to the associated asthma symptoms (Rudell et al., 2012), leading to exercise avoidance (Ram et al., 2005). Furthermore, the perception of normal indicators of physical exertion as symptoms of asthma is a common misconception (Rietveld et al., 2010, Williams et al., 2010), which, when exacerbated by a lack of fitness (McNarry et al., 2014a), leads to a greater manifestation of the apparent symptoms of asthma, resulting in further avoidance of exercise and a vicious negative cycle. 
Exercise is associated with extensive and well-recognised benefits in healthy populations (Petersen \& Pedersen, 2005), with further health benefits for those with asthma such as reduced severity and increased control (Andrade et al., 2014, van Veldhoven et al., 2001). Despite some children with asthma recognising that regular exercise is associated with improved control of their asthma and enhanced physical self-perceptions (Chiang et al., 2006), exercise is underutilised as a treatment for improved quality of life and asthma control. Previous research has shown that exercise interventions that elicit improvements in body composition and cardiorespiratory fitness are also associated with improved asthma symptoms (Carson et al., 2013, Eichenberger et al., 2013). Such improvements in asthma symptoms may be due to the increased cardiorespiratory fitness leading to a reduced ventilatory requirement for exercise (Milgrom \& Taussig, 1999, Wanrooij et al., 2014). Furthermore, given that low physical activity has been associated with poor asthma control (Dogra et al., 2011), it has been suggested that such improvements to asthma are possibly related to higher physical activity levels induced via such an intervention (Carson et al., 2013, Eichenberger et al., 2013).

There is currently no consensus in the literature as to whether children and adolescents with asthma participate in as much physical activity as their healthy peers (Berntsen, 2011, Welsh et al., 2004). Previous estimations of physical activity have largely relied on self-reports which are poorly correlated with objective measures of physical activity (Tsai et al., 2012). Furthermore, current evidence regarding the fitness levels of those with asthma is equivocal, with some studies finding those with asthma to have poorer fitness (McNarry et al., 2014a, Vahlkvist \& Pedersen, 2009, Villa et al., 2011), whilst others show no difference between those with asthma and their healthy peers (Berntsen et al., 2009, Pianosi \& Davis, 2004, Santuz et al., 1997). These discrepancies between studies may partially be due to estimations from field-based measures which are subject to significant inaccuracies dependent on self-motivation and peer influence (Cairney et al., 2008). Moreover, even in studies which have utilised peak oxygen uptake $\left(\dot{V} \mathrm{O}_{2}\right)$ as a measure of aerobic fitness, may be limited as the applicability of this measure to functional capacity during activities of daily living has been questioned (Jones, 2006, Matos \& Winsley, 2007). Indeed, there is a lack of information regarding the influence of 
asthma on the other parameters of aerobic fitness, such as the gas exchange threshold (GET) and mean response time (MRT).

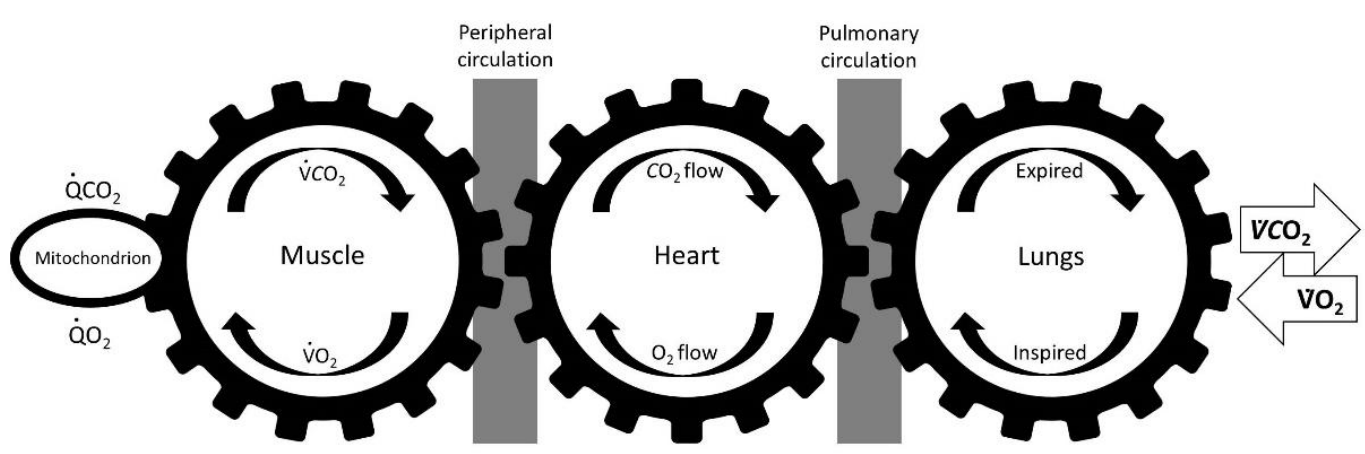

$\dot{\mathrm{Q} C O}_{2}=$ Carbon dioxide production; $\dot{\mathrm{Q}}_{2}=$ Oxygen delivery; $\dot{\mathrm{VCO}}_{2}=$ Carbon dioxide output; $\dot{\mathrm{V}}_{2}=$ Oxygen uptake.

Figure 1.1. Schematic showing the pathway of oxygen from the atmosphere to its site of utilisation within the muscle mitochondria and the numerous areas which may cause derangements in oxygen delivery. Redrawn from Wasserman et al. (1994).

Importantly, whilst previous research has assessed the peak $\dot{V} \mathrm{O}_{2}$ of adolescents with asthma (Ahmaidi et al., 1993, Counil et al., 2003), studies which have found differences (Vahlkvist \& Pedersen, 2009, Villa et al., 2011), have not sought to evaluate where derangements may occur. It is well known that with exercise comes a coordinated function of the lungs, heart and muscles to support the increase in demand for $\mathrm{O}_{2}$. Air is drawn in through the lungs via the airways $\left(\dot{V} \mathrm{O}_{2}\right)$, it is here $\mathrm{O}_{2}$ diffuses through the alveolar membrane into the blood, where it is absorbed by the haemoglobin. The cardiovascular system then delivers the oxygen to the muscles. Whilst the description is very simplistic and depicted by Wasserman's gears in Figure 1.1, it is easy to see that derangements in one or more of the systems can account for problems with $\mathrm{O}_{2}$ delivery or utilisation.

Oxygen uptake kinetics, which reflect the dynamic response to an instantaneous change in the metabolic demand (Grassi et al., 1996, Krustrup et al., 2009), may arguably be the best way to measure derangements in the $\dot{V} \mathrm{O}_{2}$ pathway. It is known that almost all diseases which affect at least one of the systems highlighted in 
Wasserman gears (Figure 1.1) inherently slow the $\dot{V} \mathrm{O}_{2}$ kinetic response. This thesis, therefore aims to measure, in addition to $\dot{V} \mathrm{O}_{2}$ kinetics, heart rate kinetics (assessing $\mathrm{O}_{2}$ delivery) and deoxyhaemoglobin $\left[\mathrm{HHb}\right.$ ] kinetics (assessing $\mathrm{O}_{2}$ utilisation at the mitochondria) to better evaluate the derangements, if any, in the $\mathrm{O}_{2}$ pathway caused by asthma. It is hypothesised that asthma, a respiratory disease, will slow the $\dot{V} \mathrm{O}_{2}$ kinetics due to an impairment in ventilation and gas exchange $\left(\mathrm{O}_{2}\right.$ delivery), and will not affect the $\mathrm{O}_{2}$ utilisation. Therefore, measures of large airway obstruction (Forced Expiratory Volume in one second $\left(\mathrm{FEV}_{1}\right)$, forced vital capacity (FVC), $\mathrm{FEV}_{1} / \mathrm{FVC}$ ) estimated small airway obstruction (Forced Expiratory Flow between 25-75\% of vital capacity) and airway inflammation (Fractional exhaled nitric oxide) will also be evaluated.

Conventionally, studies seeking to enhance the aerobic fitness of those with asthma have focussed on moderate-intensity, continuous exercise training. However, this form of exercise is often considered monotonous, which is synonymous with poor adherence (McNarry et al., 2015b) and is time consuming. Furthermore, continuous exercise has been suggested to provoke asthma attacks (Sidiropoulou et al., 2007). Conversely, it has been suggested that intermittent exercise may not provoke asthma and might aid in improving adolescents' tolerance of exercise, increasing endurance (Del Giacco et al., 2015). This highlights the potential utility of high-intensity interval training (HIIT), which is suggested to be a time-efficient method of exercise that can elicit significant improvements in both cardiorespiratory fitness and body composition in children (Costigan et al., 2015, Eddolls et al., 2017). This potential to improve body composition and Body Mass Index (BMI) through HIIT (Racil et al., 2016, Tjonna et al., 2009) is especially pertinent to those with asthma in which a greater prevalence of overweight and obesity has been reported relative to their agematched peers (McNarry et al., 2014a, Vahlkvist et al., 2010). Indeed, such observations have led to suggestions of a new asthma phenotype associated with obesity (Wenzel, 2012).

Despite the positive findings for HIIT, it is pertinent to note the potential safety considerations. Previously HIIT was considered unsafe for non-athlete populations due to risk of injury (MacDonald \& Currie, 2009). Although the use of HIIT is limited in asthma populations, participants were found to tolerate the exercise well 
(Counil et al., 2003, Latorre-Roman et al., 2014). The intermittent nature of HIIT may also facilitate a decrease in end expiratory lung volume during the resting phase (Beauchamp et al., 2010), reducing the risk of an asthma attack. However, despite widespread interest in HIIT, little is known about the effect of HIIT in children or adolescents, specifically short aerobic interval training, and particularly its interaction with asthma.

An intervention in children and adolescents is ideally located within the school setting as it is where they spend majority of their waking hours (Kriemler et al., 2011). Indeed, school based physical activity interventions have been found to lead to increases in overall physical activity and positive effects on aerobic fitness (Kriemler et al., 2011, van Sluijs et al., 2007). Moreover, the use of a school setting for the intervention provides an opportunity to reach the asthma population without having to stigmatise or discriminate (Kriemler et al., 2011). Therefore, the aim of this thesis is to assess differences between adolescents with and without asthma (Study 2), design (Study 1), implement and evaluate a school based HIIT intervention (Study $3 \& 4$ ) in adolescents with asthma.

\subsection{Thesis structure}

This thesis contains seven chapters with the central theme addressing the influence of high-intensity interval training (HIIT) on adolescents with asthma. Chapter 1 outlines the initial introduction detailing the justification for the aims of this thesis. Previous literature is reviewed and summarised in Chapter 2. The topics addressed in the literature review include asthma and its associations with obesity, physical activity and fitness. Previously utilised exercise interventions are also discussed to aid in the formation of a school-based HIIT intervention. Further discussion around measures of fitness is also reviewed for the inclusion of more intricate measures of cardiorespiratory fitness in this thesis. Chapter 3 outlines the general methods of the studies. Four experimental chapters are presented within Chapters 4 to 7 with their aims outlined below. Finally, a synthesis of the four studies discussing the main findings is given within Chapter 8 . 
Experimental study aims:

Chapter 4 To elicit the views of adolescents, with and without asthma, about exercise and asthma, and the perceived benefits of, and barriers to, participation

To inform the design of a future high-intensity interval training exercise intervention to improve asthma control

Chapter 5 To investigate the influence of asthma on the submaximal and maximal parameters of cardiorespiratory fitness in adolescents

To elucidate the potential relationship between cardiorespiratory fitness, physical activity, body mass and asthma

Chapter 6 To investigate the effectiveness of an inclusive field-based six-month HIIT intervention on aerobic fitness, BMI, lung function and quality of life in adolescents with asthma

Chapter 7 To investigate the influence of asthma and HIIT, and their interaction, on the dynamic $\dot{V} \mathrm{O}_{2}$ response in adolescents 


\section{Thesis study map}

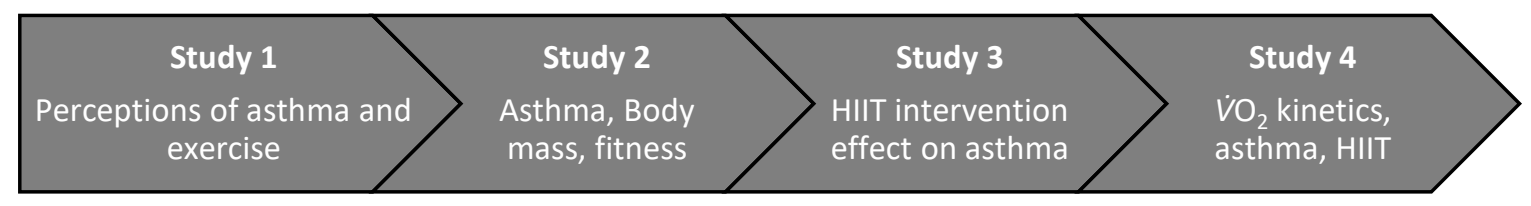

Throughout the thesis, this study map will reiterate the aims of each experimental chapter and the key findings for future chapters 


\section{CHAPTER 2}

\section{LITERATURE REVIEW}




\section{Chapter 2: Literature Review}

\subsection{Introduction}

Asthma has been found to be influenced and potentially caused by lack of physical activity and obesity (Chen et al., 2013, Papoutsakis et al., 2013). Due to the high cost of asthma there is a need to reduce severity and symptoms of the condition through a non-pharmacological method (Chakir et al., 2002, Holgate, 2011, Sumi \& Hamid, 2007). One such suggested additional therapy is exercise training, which has been found to be beneficial in child asthma management (Carroll \& Sly, 1999), however, it has not been extensively studied (Chandratilleke et al., 2012) and, therefore, there is a need to explore further. Previously, studies have utilised moderate-intensity continuous training with some success, however such protocols may cause higher risk for participants with asthma due to the onset of exercise induced bronchoconstriction (Del Giacco et al., 2015).

The topics of asthma, obesity, physical activity and fitness are largely beyond the scope of this thesis and therefore are not reviewed in detail but are set in wider context. The purpose of this literature review is to assess and evaluate the influence of asthma on obesity, physical activity and cardiorespiratory fitness.

Literature was acquired using PubMed, CINAHL and the Cochrane library; databases were searched from inception to May 2018. The four main search terms were asthma AND child AND exercise AND intervention. A mix of title, abstract and main body text searches were used. Non-indexed randomised controlled trials were also retrieved using hand searching from those selected papers' reference lists.

\subsection{Asthma}

The severity of asthma can be classified as intermittent or persistent (mild, moderate or severe), which takes into consideration the presence of diurnal and nocturnal symptoms, frequency of exacerbation, necessity of medication, pulmonary function and physical activity limitation (Global Initiative for Asthma, 2017). However, as 
asthma is a heterogeneous condition, there is no gold standard definition and no specific single diagnostic test and, therefore, the clinical history along with corroborating tests are required to confirm the diagnosis and determine the potential response to medication (British Thoracic Society, 2016). Indeed, the diagnosis of asthma often also relies on symptoms which are not specific to asthma and therefore overlap with other conditions; for asthma, an objective confirmation of reversible airways obstruction is required, usually by assessing bronchodilator response or bronchial hyperresponsiveness (Taylor et al., 2008).

Asthma represents a significant burden for patients, their families, healthcare systems and the economy (Akinbami et al., 2012, Doz et al., 2013). There is currently no cure for asthma and it is associated with over 1000 deaths per year in the UK, roughly 2\% children (Mukherjee et al., 2016), 46\% of which could be avoidable if the appropriate asthma management guidelines were followed. This highlights the need to find cost-effective ways to reduce symptoms and improve asthma control.

The primary aim of asthma treatment is to achieve and maintain control, reduce future risks and, essentially, enable that person to lead a life without restriction (Bacharier et al., 2008, Global Initiative for Asthma, 2017, Reddel et al., 2009). The British Thoracic Society define complete control as no daytime symptoms, no nighttime awakening, no need for rescue medication, no asthma attacks, no limitations on activity including exercise, normal lung function and minimal side effects from medication (British Thoracic Society, 2016). Asthma management should be adjusted in a continuous cycle, with regular control assessments in order to gain and maintain asthma control, with little or no impairment to the person. Fundamental to this process is the identification of the lowest step of medication, minimising costs and possible side-effects (Global Initiative for Asthma, 2017). However, despite the plethora of effective medications, many children and adolescents still do not have adequately controlled asthma (Fuhlbrigge et al., 2006, Gustafsson et al., 2006), most likely due to non-adherence to their prescribed medication (Vrijens et al., 2012). Indeed, it was found that only 1 in 20 children with asthma in Western Europe met the criteria outlined by GINA for asthma control (Rabe et al., 2000). A lack of asthma control has knock on effects for quality of life and daily physical activity, 
causing an increased burden on family and society (Pijnenburg et al., 2015). Furthermore, as recognised by many experts in the field, treatment options for asthma, namely bronchodilators, anti-inflammatories and biologics in selected patients are relatively limited and therefore there is a need for other therapies, including non-pharmacological treatments, to aid in management (Chakir et al., 2002, Holgate, 2011, Sumi \& Hamid, 2007).

\subsection{Asthma phenotypes and triggers}

As asthma varies throughout life, and especially during childhood, a key research focus is the identification of asthma phenotypes (recognisable clusters of demographic, pathological or clinical characteristics, Bousquet et al., 2011, Spycher et al., 2010, Wenzel, 2012) or endotypes (specific characteristics that explain the observable properties of a phenotype, Lotvall et al., 2011) for more individually targeted management or personalised medicine. Some phenotype-guided treatments are available for those with more severe asthma, however, treatment responses or clinical patterns show no strong relationship with specific pathological features (Anderson, 2008). Therefore, more research is required on asthma phenotyping to understand different treatments and the clinical utility of phenotypic classifications in asthma (Global Initiative for Asthma, 2017). Phenotypes have been described previously based upon the time of first wheeze, presence of allergic sensitisation, eosinophilic or non-eosinophilic inflammation (Saglani \& Bush, 2008), response to treatment (Szefler et al., 2002, Szefler et al., 2005), asthma severity (Brasier et al., 2010) and allergic comorbidities. Some of the most commonly recognised phenotypes include: Allergic asthma, non-allergic asthma, late-onset asthma, asthma with fixed airflow limitation, asthma with obesity (Global Initiative for Asthma, 2017), or virus-induced, allergen-induced, exercise-induced or obesity-related asthma (Riner \& Sellhorst, 2013).

Physical activity is associated with increased exposure to environmental factors due to increased ventilation, especially in cold air, chlorinated swimming pools, in seasons with high levels of allergens or in polluted areas (Carlsen et al., 2011, Del Giacco et al., 2012). Furthermore, water loss and cooling of airways through 
increased ventilation during physical activity may cause increased airway resistance in addition to immune responses (Del Giacco et al., 2012), which may aggravate asthma and asthma symptoms (Carlsen et al., 2011, Del Giacco et al., 2012). Increased eosinophilic airway inflammation, indicated by levels of exhaled nitric oxide, have been reported in association with time spent in vigorous physical activity in non-asthma adolescents (Sachs-Olsen et al., 2013), along with the newly reported sports asthma phenotype (Couto et al., 2015), suggesting endurance exercise may be a possible risk factor for asthma.

Exercise is one of the major triggers for asthma symptoms and can lead to exerciseinduced bronchoconstriction (EIB). The manifestation of EIB is characterised by shortness of breath which results from a reversible narrowing of the airways (Basaran et al., 2006, Grzelewski \& Stelmach, 2009, van Leeuwen et al., 2011). The American Thoracic Society guidelines define EIB as a drop of $>10 \%$ in Forced Expiratory Volume in one second $\left(\mathrm{FEV}_{1}\right)$ post exercise (Ross, 2003). Exercise induced bronchoconstriction is common in adolescents with asthma and the occurrence of symptoms has been found to prevent practice of physical activity, especially in those with more severe asthma (Lang et al., 2004). The mechanisms of EIB in asthma remain unclear, but it is likely to be caused by an increase in ventilatory rate and concomitant drying and cooling of the airways which can trigger a pro-inflammatory response (Weiler et al., 2007).

The characteristics of the asthma obese phenotype include high medication use, high admission rates to hospital for asthma-related problems and a decreased quality of life (Holguin et al., 2011, Novosad et al., 2013, Taylor et al., 2008). A previous cluster analysis has suggested that obese asthma is a distinct phenotype and it is not only characterised by poor asthma control but also that it is predominant in females and has an absence of eosinophilic airway inflammation (Haldar et al., 2008). In addition, the obese asthma phenotype has also been suggested to be dominant among children (Lang et al., 2011, Papoutsakis et al., 2013). It has been shown that overweight and obese asthma sufferers are more likely to report exercise as a trigger than their normal weight peers (Wright et al., 2010). When looking specifically at children with no history of asthma it was found that obesity may contribute to an increased frequency of EIB (Ulger et al., 2006) or higher levels of bronchospasm 
post exercise testing (Haas et al., 1987, Kaplan \& Montana, 1993). In addition to increased frequency, obese children with asthma had increased severity of EIB in comparison to their normal weight asthma peers (Baek et al., 2011, Lopes et al., 2009). Moreover, in a dietary weight loss study, children who were overweight or obese with asthma were found to have reductions in severity of EIB, represented by a reduced fall in $\mathrm{FEV}_{1}(30 \%$ vs. $21 \%, \mathrm{P}<0.05)$ and improvements in quality of life (4\%, van Leeuwen et al., 2014). The use of weight loss interventions, possibly through physical activity, may aid in alleviating asthma symptoms in children and adolescents.

\subsection{Asthma medication}

Pharmacological management of asthma aids in the maintenance of control of the disease. Usually on first diagnosis of asthma, patients are prescribed a short acting bronchodilator to aid in the relieving the symptoms (British Thoracic Society, 2016). For mild asthma or infrequent wheeze, the occasional use of reliever therapy may indeed be the only treatment required. There are numerous short-acting bronchodilators, but the most commonly prescribed is the short-acting $\beta 2$ agonists (SABA) as they work more rapidly than the alternatives and with fewer side effects (WHO, 2018). Little or no requirement for use of a SABA inhaler is associated with good asthma control; in contrast, if the patient is non-adherent, not using SABA may result in poor control. In the case of using SABA more than 3 times per week or waking due to asthma symptoms during the night, a preventer treatment is considered. To prevent the symptoms of asthma from occurring the most common form of preventer treatment is inhaled corticosteroids (ICS) as they are the most effective in both adults and children for reducing inflammation and thus achieving overall treatment goals (Scottish Intercollegiate Guidelines Network, 2014, Teper et al., 2004). With mild asthma or good control of the disease, using ICS can be recommended once a day but it is more commonly used twice daily (WHO, 2018). On occasion, asthma patients may not have their condition under control using ICS and therefore add-on therapies are suggested prior to increasing ICS above a low dose (200-1000 and 400 micrograms in adults and children, respectively; Scottish Intercollegiate Guidelines Network, 2014). Long-acting $\beta 2$ agonists (LABA) can be 
used in addition to ICS and are the first choice for add-on therapy to increase lung function and decrease asthma attacks in adolescents (Scottish Intercollegiate Guidelines Network, 2014).

Short-acting bronchodilators with or without LABA and Leukotriene Receptor Antagonists (LTRAs) are the most common treatment for EIB, but nonpharmacological strategies such as warming up adequately or increasing fitness have also been reported to be effective (Minic \& Sovtic, 2017). The use of asthma medication should control exercise induced symptoms and allow those with asthma to participate in habitual physical activity and exercise (Minic \& Sovtic, 2017). Indeed, for most asthma sufferers, EIB is associated with poorly-controlled asthma and their medication should therefore be reviewed (British Thoracic Society, 2016). Furthermore, it has been suggested that $75-80 \%$ of people with asthma who participate in exercise without anti-inflammatory medication may experience an asthma attack (Crimi et al., 1996). However, as long as a bronchodilator is administered 15-30 minutes prior, exercise for people with EIB is considered safe (Parsons et al., 2013). It is important to note that education is needed, since the majority of those with asthma are not aware that medication should be taken prior to exercise (Walker \& Reznik, 2014).

Whilst there is speculation that SABA can improve exercise performance in nonasthma populations, Kindermann \& Meyer (2006) found no ergogenic potential of $\mathrm{SABA}$ in athletes, in a systematic review. In a more recent systematic review and meta-analysis, Pluim et al., (2011) showed no significant effects of inhaled $\beta_{2}$ agonists on endurance, strength or sprint performance. However, there is some evidence, albeit weak, that systematic $\beta_{2}$-agonists may have a positive effect on performance (Pluim et al., 2011). Whilst there is no benefit to exercise performance, the use of SABA and other asthma medication aid in relieving the symptoms of asthma which may effectively "normalise" those with asthma. 


\subsection{Quality of life and asthma}

Quality of life is a broad, multidimensional concept which can include subjective evaluations of positive and negative aspects of life (The WHOQOL Group, 1998). Within research, quality of life is measured using three defined approaches: healthrelated quality of life, social indicators and subjective well-being (Cummins et al., 2004, Diener \& Suh, 1997, Michalos, 2004). The most commonly used within clinical health research is health-related quality of life (Wallander \& Koot, 2016). Health-related quality of life specifically measures a person's subjective perception of their own health, disease and disability (De Civita, 2005).

The central target for health interventions is to improve quality of life (RavensSieberer et al., 2014) and when implementing an intervention or a treatment to benefit a person's health, their quality of life should improve, or at the very least not cause it to reduce (Testa \& Simonson, 1996). There has been a decline in quality of life in adolescents over the last decade (Cui \& Zack, 2013) and therefore there is a need to measure and target quality of life during interventions.

Cardiorespiratory fitness and body mass index (BMI) have previous been shown as predictors of health-related quality of life, with a higher fitness associated with higher quality of life (Andersen et al., 2017, Morales et al., 2013, Sloan et al., 2009) and a higher BMI related to a poorer quality of life (Swallen et al., 2005, Williams et al., 2005). Therefore, these measures should be targeted in future interventions aimed at improving a population's quality of life.

In asthma studies, measures such as forced expiratory volume in one second, peak expiratory flow and symptoms can be used to assess asthma control, and, in the past, these were thought to provide insight into a person's well-being (quality of life, Juniper et al., 2004). Whilst this is certainly true for those with more severe asthma, research has shown that quality of life is a distinct component of asthma and does not correlate with asthma control (Juniper et al., 2004). In addition to health-related quality of life, asthma related quality of life can also be measured and refers to the subjective impact of asthma on a person's quality of life. The use of asthma related quality of life to describe how asthma impacts a person's daily life is now often used 
as an outcome measure (Juniper, 1997). In addition to the measures in health-related quality of life, asthma related quality of life measures physical, emotional occupational and social aspects of how asthma can impact a person's life (Juniper et al., 2010).

Previously when compared to their healthy peers, participants with asthma were reported to have a lower quality of life (Merkallio et al., 2005, Molzon et al., 2013). However, this may be due to the participants having poorly controlled asthma which may serve to reduce quality of life over and above the effects of asthma per se (Sundbom et al., 2016). One potential way of increasing the quality of life in participants with asthma may be to implement an exercise intervention. Previously, exercise interventions have been found to significantly improve asthma related quality of life (Fanelli et al., 2007, Goncalves et al., 2008, Mendes et al., 2010, Turner et al., 2010), however not in all studies (Moreira et al., 2008). Specifically, Moreira et al. (2008) found no increase, but there were no decreases reported by any study in asthma related quality of life, supporting the use of exercise interventions. Furthermore, the high baseline levels of quality of life in the Moreira et al. (2008) could have removed the ability and/or need for an increase.

\subsection{Obesity and asthma}

Obesity is defined by the World Health Organisation (WHO) as "abnormal or excessive fat accumulation that may impair health" (WHO, 2018a). Body mass index (BMI) is the easiest and most common way of determining obesity as body mass is proportional to the square root of body height. Body mass index is also the most useful measure of overweight and obesity at the population-level as it is the same for both sexes, although caution should be taken as it may not correspond to the same level of fatness in different people (Wang, 2004). Indeed, factors such as age, sex and ethnicity may influence the relationship between BMI and body fat. Furthermore, BMI cannot distinguish between excess fat, muscle or bone mass which can influence the interpretation, for example, women have greater fat mass than men with the same BMI and older adults tend to have higher fat mass in comparison to younger adults with the same BMI. Overweight is classified as having 
a BMI greater than or equal to $25 \mathrm{~kg} \cdot \mathrm{m}^{-2}$, with obesity $30 \mathrm{~kg} \cdot \mathrm{m}^{-2}$ or over. In children and adolescents between 5 and 19 years, overweight is defined as BMI-for-age greater than 1 standard deviation above the WHO Growth Reference median, with obesity being greater than 2 standard deviations. According to the Centres for Disease Control and Prevention (2009), if a child or adolescent is shown to be obese there is a $99 \%$ chance they are, however those in the overweight category can be misrepresented by increased fat free mass. However, BMI is a good indicator of body fat, but should be used more as an estimate as it does not directly measure body fat. BMI can be used in research to track weight status and as a screening tool for potential weight problems (Centres for Disease Control and Prevention, 2009).

Obesity has nearly tripled between 1975 and 2016 worldwide with overweight and obesity the fifth leading risk for global deaths (European Association for the Study of Obesity, 2018), surpassing deaths caused by people being underweight (WHO, 2018a). Approximately 1.9 billion adults worldwide are classified as overweight, of which over 650 million were classified as obese (WHO, 2018a). There are also over 340 million children and adolescents aged 5-19 years who were classed as overweight or obese in 2016 (WHO, 2018a); nearly a third of children in the UK aged between 2 and 15 years are overweight or obese (Gov, 2017). The costs associated with the increase in overweight and obesity-related illness meant the NHS spent $£ 5.1$ billion in 2014/15 (Gov, 2017).

Obesity is linked with many serious physiological health complications, such as heart disease and diabetes (WHO, 2018a), as well as mental health conditions such as depression (Luppino et al., 2010). In more recent years obesity has been suggested to be a risk factor for the development of asthma. Indeed, the increase in obesity prevalence has been accompanied by a similar increase in asthma (Delgado et al., 2008, Townsend et al., 2013). Moreover, the prevalence of asthma is also greater in the obese population (Forte et al., 2013), leading to speculation that there may be a direct relationship between the two (Mannino et al., 2002).

There is, however, a disagreement in the cause of this relationship and the degree and direction of the association between obesity and asthma (Leinaar et al., 2016). It has been suggested that increased fat mass may cause both mechanical and 
systematic inflammatory changes which can influence breathing mechanisms and airway inflammation (Jensen et al., 2011, Lucas \& Platts-Mills, 2006, Willeboordse et al., 2016). Indeed, obesity may affect lung function in a variety of different ways (Ford, 2005, Sutherland et al., 2008), including a reduction in residual volume, functional residual capacity and expiratory reserve volume. Furthermore, although obesity does not influence the $\mathrm{FEV}_{1}$ /forced vital capacity $(\mathrm{FVC}$ ) ratio (which when reduced, indicates the airway obstruction associated with asthma), it has been shown to reduce both $\mathrm{FEV}_{1}$ and FVC (Lessard et al., 2008). The reduction in FEV1 and FVC may be due to markedly increased gastric and oesophageal pressure which increases the work of breathing (Steier et al., 2014). It is worth noting, however, that not all studies found an influence of obesity on lung function in adults with asthma (Ghabashi \& Iqbal, 2006), but this may be due to the lack of age and sex matched controls.

The mechanisms responsible for both asthma and obesity are heterogeneous due to the complex nature of the conditions (Lang et al., 2004). The direction of causality between asthma and obesity remains unclear, but it has been postulated that the manifestations and treatments of asthma (corticosteroids) may increase the risk of obesity (Savas et al., 2017). Longitudinal studies, however, have demonstrated that obesity significantly increases the risk of future diagnosis of asthma (Camargo et al., 1999). Obesity is linked to an array of diseases and this could therefore require increased GP visits which may lead to a diagnosis of asthma, which may have gone undiagnosed. Although, reduction in body mass may aid in the treatment and control of asthma (Jensen et al., 2013, van Leeuwen et al., 2014), and there is therefore a need to reduce obesity in order to manage asthma related issues in children and adolescents.

Obesity has been found to reduce asthma control (Barros et al., 2011, Saint-Pierre et al., 2006) and can lead to a more difficult to control phenotype (Beuther et al., 2006). It could be postulated that this lack of control may be due to obese individuals with asthma tending to have more severe asthma which does not respond well to treatment (Dixon et al., 2010). Furthermore, increases in body weight have been found to impair drug therapy; inhaled corticosteroids have a reduced effect with 
higher body mass in both children and adults, requiring an increased dosage (Boulet \& Franssen, 2007, Camargo et al., 1999, Forno et al., 2011, Peters-Golden et al., 2006). Indeed, adults with increased weight had reduced treatment efficacy and this was associated with worse asthma control and quality of life (Lavoie et al., 2006). Moreover, obese patients with higher doses of medication were found to receive emergency treatment for acute asthma attacks more often than their normal weight peers (Rodrigo \& Plaza, 2007). Therefore, a reduction in BMI may not only aid in reducing further treatment burden and costs but also the beneficial effect of the medication.

Not only is obesity associated with an increased risk of asthma, there is also an association with increased disease severity and increased use of health care resources (Belamarich et al., 2000). Previously, dietary weight loss in overweight and obese children with asthma was found to result in reductions in severity of EIB along with improvements in quality of life and lung function (Dixon et al., 1999, van Leeuwen et al., 2014). Specifically, in obese children with asthma, weight-loss alone (with no exercise component) interventions showed improvements in lung function, inflammatory biomarkers and EIB (Jensen et al., 2013, van Leeuwen et al., 2014). Reducing obesity is not only a potential benefit for reducing asthma symptoms, but will aid in saving lives as obesity doubles the risk of dying prematurely (Franks et al., 2010). Exercise can be an effective means of improving body composition in young obese individuals (Lau et al., 2015), with improvements in body mass and body fat percentage (Racil et al., 2016, Tjonna et al., 2009). Indeed, increasing the energy expenditure may aid in combating the energy intake, as previously those who tried to restrict their diet in a study were more successful in increasing the physical activity (DeLany et al., 2014); possibly due to committing to the intervention and adherence to both targets. In summary, weight loss appears to be an effective treatment for the obese asthma phenotype, however studies to date have been limited and mechanisms remain to be fully elucidated. Therefore there is a need to combat obesity in order to aid in reducing asthma and asthma symptoms through a nonpharmacological treatment.

As the fundamental cause of obesity and overweight is an energy imbalance between calories consumed and calories expended, physical activity may aid in reducing BMI 
(Walders-Abramson et al., 2009). Indeed, the association between reduced physical activity and obesity has been found in several studies (Belcher et al., 2015, Rauner et al., 2013). Not all studies concur, however, as obesity may be caused by an increase in energy intake and not by a decrease in physical activity (Metcalf et al., 2012). Obesity can lead to physical inactivity in both children and adolescents and sedentary children with asthma were more likely to be overweight (Trost et al., 2001). In addition, comorbidities of asthma and obesity may also contribute to furthered sedentary behaviour which can lead to worse asthma symptoms (Beckett et al., 2001, Camargo et al., 1999). Moreover, overweight children and adolescents with EIB were found to have reduced time in vigorous physical activity in comparison to their normal weight peers (Anthracopoulos et al., 2012), further to this they also spent less time in lower intensity physical activity (Anthracopoulos et al., 2012). As those with asthma who are overweight often struggle with physical activity, a vicious negative cycle may form whereby their energy demand will not outweigh their energy intake and therefore they further increase in body mass (Ford, 2005, Guerra et al., 2002). The suggestion that asthma and obesity are comorbid resulting in reduced physical activity and further weight gain, suggest that increasing physical activity may aid in the management of both conditions (Fedele et al., 2014, Lawson et al., 2013).

\subsection{Physical activity and asthma}

Physical activity is defined as any bodily movement produced by the skeletal muscles that requires energy expenditure (Caspersen et al., 1985). Being physically inactive is the fourth highest risk factor in the world for all-cause mortality (WHO, 2018b). The measurement of physical activity is derived from estimated energy expenditure measures of step count, accelerometer counts or heart rate (Montoye, 2000). Physical activity may also be measured using subjective measures of exhaustion, observation or self-report of activities undertaken (Montoye, 2000). Objectively measuring physical activity often provides a more valid measure of physical activity, however, it is costly and cannot be used during contact or watersports leading to missing data, or the reliance on self-report to fill in the gaps. The ActiGraph GT3X+ accelerometer (ActiGraph, Pensacola, FL, USA), which can 
record the quantity, intensity and frequency of body movement, is possibly the leading instrument for measuring energy expenditure (Butte et al., 2010). The accelerometer is worn most commonly on either the waist or the wrist and is typically used to continuously monitor movement across a seven day period (Rich et al., 2013). Physical activity intensities are defined by predetermined "cut-points" for objective measures, which are different for children and adults. These cut-points categorise by intensity, using groupings such as light, moderate or vigorous and reported as frequency, type and mode of activity (Montoye, 2000).

The international physical activity recommendations for children and adolescents are to spend at least 60 minutes per day in moderate-to-vigorous physical activity (Department of Health, 2011), with NICE guidelines recommending those with asthma participate in equal amounts while focusing on managing their condition (NICE, 2018). The benefits of physical activity are numerous, including increases in fitness, quality of life, psychological factors and decreases in obesity (Janssen \& Leblanc, 2010). Furthermore, there is evidence that physical activity and exercise are linked to academic performance (Donnelly et al., 2016). In addition to these benefits, there are further well documented benefits for those with asthma. Specifically, increased physical activity results in increased cardiorespiratory fitness which has been reported to be associated with improved psychological functioning in children with asthma (Pianosi \& Davis, 2004). Physical activity can also reduce dyspnoea and the dose of inhaled corticosteroids, intensity of EIB and exacerbations (Basaran et al., 2006, Fanelli et al., 2007, Vahlkvist et al., 2010). Moreover, one study reported a significant albeit weak correlation $(\mathrm{r}=0.11, \mathrm{P}<0.01)$ between low physical activity and the development of asthma (Rasmussen et al., 2000); in addition, young children with increased television screen time (increased sedentary level; > 2 hours per day) were twice as likely to develop asthma by late childhood as those who were limited to 1-2 hours of screen time (Sherriff et al., 2009).. Indeed, outlined by a metaanalysis (Lochte et al., 2016), three cohort studies revealed that there is up to a $35 \%$ increased risk of new onset asthma and/or wheezing in children with low levels of physical activity (Sherriff et al., 2009, Islam et al., 2009 and Vogelberg et al., 2007). Furthermore, three cross-sectional studies showed significant positive associations 
between low physical activity and new-onset asthma (Lang et al., 2004, Mitchell et al., 2013 and Priftis et al., 2007).

There are, however, increasing numbers of adolescents who do not hit the recommended level of physical activity per day (Willeboordse et al., 2016). Specifically, studies involving healthy children have reported decreasing physical activity with age (Corder et al., 2013, Corder et al., 2016), with as many as $80 \%$ of adolescents worldwide not reaching the recommended physical activity levels (Hallal et al., 2012). Similarly, physical activity levels in children with asthma decrease with age, with this decrease more pronounced in females (Chen et al., 2001, Yiallouros et al., 2015). Equally, some children with asthma may get discouraged from physical activity by negative self-perceptions of their own athletic ability (Glazebrook et al., 2006, Pianosi \& Davis, 2004, Tsai et al., 2012). However, the desire to meet socially acceptable norms may partially counter negative perceptions, with boys trying to downplay their asthma symptoms, although girls may in fact lower their expectations of physical activity (Rhee et al., 2007). Therefore, physical activity is not only an important part of self-management, with numerous benefits, for adolescents with asthma (Welsh et al., 2005), but a possibly preventative measure. Furthermore, there is a need to increase physical activity and maintain it throughout adolescents into adulthood, especially as childhood physical activity has been shown to influence habits of physical activity in adulthood (Blair et al., 1989, Dumith et al., 2011).

There are many studies examining the effects of asthma on physical activity, however, there is some controversy as to whether children and adolescents with asthma participate in as much as their non-asthma peers (Berntsen, 2011, Welsh et al., 2004). Some studies showed similar levels of physical activity between children and adolescents with and without asthma (Berntsen et al., 2009, Eijkemans et al., 2008, Nystad, 1997, Vahlkvist et al., 2010, van Gent et al., 2007, Welsh et al., 2004), but others demonstrated that those without asthma participated in significantly more (Glazebrook et al., 2006, Kitsantas \& Zimmerman, 2000, Lang et al., 2004, Lucas \& Platts-Mills, 2005, Sousa et al., 2014, van Veldhoven et al., 2001, Villa et al., 2011) with suggestions that found the difference according to asthma status may be greater in girls (Crosbie, 2012, Wanrooij et al., 2014, Yiallouros et al., 2015). A possible 
reason for these discrepancies between studies may be the use of self-reported physical activity, which requires participants to have an understanding of what constitutes differing levels of physical activity. These measures are therefore less accurate; intensity is often over reported, and a low correlation with objective physical activity measurements has been demonstrated (Tsai et al., 2012). It is perhaps worth noting that in a recent meta-analysis based solely on objective recordings of physical activity, no differences were found between those with and without asthma (Cassim et al., 2016). The differences in physical activity between children and adolescents with and without asthma may therefore be due to the severity of the condition as there is an association between the severity of asthma and lower physical activity levels (Lang et al., 2004, Nystad et al., 2001, Tsai et al., 2012, Vahlkvist et al., 2010). Indeed, previous studies have shown that when asthma symptoms are well controlled, there are no differences between activity levels between those with and without asthma (Sousa et al., 2014, Vahlkvist et al., 2010). Moreover, when asthma control was increased and severity reduced, physical activity was also shown to increase (Lang et al., 2004, Vahlkvist et al., 2010). In addition, although some studies report no differences between those with and without asthma, it has been suggested that physical activity levels in children with asthma may be "artificially high" as active children are more likely to be diagnosed with asthma (Nystad, 1997, Williams et al., 2008b); as less active children may not be provoking their non-diagnosed asthma symptoms. This artificially high physical activity level may be paired with suggestions that adolescents with undiagnosed asthma may avoid physical activity participation in order to avoid asthma symptoms (Williams et al., 2008a), which may explain the lack of difference between those with and without asthma. Therefore achieving good asthma control may allow those with asthma to perform the same amount of physical activity as their non-asthma peers (Sousa et al., 2014). Moreover, participating in more physical activity will aid in conditioning the person thereby potentially further improving asthma control.

Physical activity is an integral part of adolescent lives, including friendship, pleasure and the ability for those with asthma to seek normality in front of peers (Protudjer et al., 2009). The discomforts of social and emotional issues of being different have also been reported as more unpleasant than asthma symptoms appearing during 
physical activity (Walsh et al., 2008). However, previous research separate participants with asthma which may further stigmatise them instead of using an inclusive intervention. Although, fear of asthma symptoms and attacks may act as a barrier to physical activity, especially in front of peers (Rudell et al., 2012) and therefore children with asthma can end up avoiding it completely (Ram et al., 2005). This fear is unfounded as if asthma is well-controlled, through use of long-term controller medication, it may result in increased physical activity in comparison to their peers (Vahlkvist et al., 2010). Indeed, contrary to common misconceptions, no adverse effects were reported in three recent systematic reviews (Carson et al., 2013, Eichenberger et al., 2013, Wanrooij et al., 2014), indicating that physical activity is safe, feasible and can be recommended for children and adolescents with asthma.

To summarise, physical activity is recommended for children with asthma (Chandratilleke et al., 2012, Wanrooij et al., 2014) and if their asthma is wellcontrolled a physically active lifestyle is feasible (Berntsen, 2011). Due to the equivocal findings in adolescents with asthma physical activity, more research is required in objective measures of physical activity. Furthermore, perceptions of nonasthma participants may hinder the participation in physical activity of those with asthma, possibly leading to a more sedentary lifestyle further reducing asthma control. Indeed, further research is required to assess the perceptions of both those with and without asthma on asthma and physical activity participation. Finally, in order to increase physical activity among asthma, further studies should utilise inclusive interventions to avoid stigmatising asthma participants. Furthermore due to the aforementioned benefits of physical activity for adolescents with asthma, exercise, the planned, structured and repetitive form of physical activity, with the intention of improving or maintaining fitness, has been proposed as a means of increasing physical activity.

\subsection{Fitness and asthma}

Exercise has been used previously to improve health and wellbeing in many different populations (Anderson et al., 2016, Farina et al., 2014, Haskell et al., 2007, Janssen \& Leblanc, 2010). Exercise is associated with extensive and well-recognised benefits 
in healthy populations and protects against all-cause mortality, inclusive of heart disease, obesity and Type II diabetes mellitus (Petersen \& Pedersen, 2005). Furthermore, there is increasing evidence that exercise and an increase in fitness can elicit further benefits for those with asthma (Andrade et al., 2014, van Veldhoven et al., 2001). Cardiorespiratory fitness is a measure of the ability of the respiratory and circulatory systems to supply oxygen to the muscles over a prolonged period of time during physical activity. Due to the derangements of the airways associated with asthma, low cardiorespiratory fitness could indeed play a role in worse asthma severity, with high fitness being shown to relate to fewer asthma symptoms (Lucas \& Platts-Mills, 2005, Rasmussen et al., 2000, van Veldhoven et al., 2001) and low physical fitness associated with poor asthma control (Dogra et al., 2011). Furthermore increased cardiorespiratory fitness may aid in increasing exercise tolerance and capacity in children with asthma and therefore increase the threshold for inducing EIB (Milgrom \& Taussig, 1999). Improvements in asthma symptoms could be due to the proposition that increased cardiorespiratory fitness may lead to an improved ventilator equivalent for a given exercise intensity below maximal exercise, this decrease in ventilation rate may reduce airway aggravation (Milgrom \& Taussig, 1999, Wanrooij et al., 2014). Increased fitness has also been shown to increase health-related quality of life including social, emotional, physical and cognitive functioning (Flapper et al., 2008). Therefore an exercise intervention may aid in reducing asthma severity and symptoms as a non-pharmacological treatment (Matsumoto et al., 1999, Wanrooij et al., 2014, Welsh et al., 2005).

Despite some children with asthma recognising that regular exercise is associated with improved control of their asthma and enhanced physical self-perceptions (Chiang et al., 2006), physical activity and exercise, as tools to ameliorate lack of asthma control, are often avoided and are underutilised as a treatment to improve quality of life. Perceptions of asthma and exercise are later discussed in Chapter 4. Furthermore, because of this association between asthma and exercise, the attribution of normal indicators of physical exertion - increased breathing, heart rate and sweating - to symptoms of asthma is a common misconception (Rietveld et al., 2010, Williams et al., 2010), which, when exacerbated by a lack of fitness (McNarry et al., 2014a), leads to a greater manifestation of the apparent symptoms of asthma, 
resulting in further avoidance of exercise and a vicious negative cycle. Moreover, it has previously been found that peripheral muscle deconditioning may be a result of physical inactivity caused by breathlessness (Laveneziana et al., 2006). Deconditioning can result in further breathlessness due to the increased energy demand from atrophied muscles (Swallow et al., 2007). This knock on effect results in a negative cycle of exercise avoidance further deconditioning the muscles (Mioxham \& Jolley, 2009). However, the use of exercise training may reduce these perceptions of breathlessness through increased respiratory muscle strength (Carson et al., 2013). Furthermore, a higher perceived competence of physical activity in adolescents with asthma is associated with increased fitness (Pianosi \& Davis, 2004). Finally, a study examining a cross-section of ages between childhood and adulthood suggested that increased cardiorespiratory fitness was associated with a reduced risk of asthma (Guldberg-Moller et al., 2015). Indeed, exercise may aid in reducing airway inflammation providing a protective effect against asthma (Eijkemans et al., 2012, Ford, 2002) and improve the overall condition of the bronchioles (Eijkemans et al., 2012).

Adolescents with asthma are often perceived as having lower cardiorespiratory fitness, however, the current literature is conflicting. Indeed, whilst some studies show participants with asthma to have poorer fitness than their healthy peers (McNarry et al., 2014a, Vahlkvist \& Pedersen, 2009, Villa et al., 2011), others show no differences (Berntsen et al., 2009, Pianosi \& Davis, 2004, Santuz et al., 1997). The differences between the studies may potentially be explained by methodological variations. Each of the studies may have used participants with differing severities of asthma and therefore, differences may be misrepresented; however, it has been shown that there is no association between severity of asthma and cardiorespiratory fitness (Pianosi \& Davis, 2004). A more likely explanation for the discrepancies between the studies are the exercise tests used to assess cardiorespiratory fitness. Indeed, cardiorespiratory fitness as estimated by field based measures are subject to substantial inaccuracies due to motivation and peer influence (Cairney et al., 2008). 


\subsection{Fitness testing}

It is widely accepted that the best single measure for an individual's aerobic fitness is maximal oxygen uptake $\left(\dot{V} \mathrm{O}_{2 \max }\right)$, which represents the capacity of the pulmonary, cardiovascular and muscular systems to uptake, transport and utilise $\mathrm{O}_{2}$ (Poole et al., 2008). Although $\dot{V} \mathrm{O}_{2 \max }$ in adults can usually be identified from an exercise test by a plateau in $\dot{V} \mathrm{O}_{2}$ despite increasing work rate (Armstrong \& Welsman, 1994), issues still confound its utility in paediatric populations, as only a minority display this plateau in $\dot{V} \mathrm{O}_{2}$ (Armstrong et al., 1996, Armstrong \& Welsman, 1994, Barker et al., 2011). Therefore, in paediatric populations, peak $\dot{V} \mathrm{O}_{2}$, which is defined as the highest $\dot{V} \mathrm{O}_{2}$ attained during an exhaustive exercise test is accepted as the standard outcome measure for children and adolescents (Rowland et al., 1994).

Studies investigating peak $\dot{V} \mathrm{O}_{2}$ are further hindered by not accounting for a possible influence of body size, which can limit inter-study comparisons. It is especially important to account for body size during puberty around peak height velocity due to increases in body mass outweighing increases in peak $\dot{V} \mathrm{O}_{2}$. During puberty, when making peak $\dot{V} \mathrm{O}_{2}$ relative to body mass it will penalise those who are more mature and heavier (Armstrong, 2007); Albrecht et al. (1993) demonstrated the negative correlation between body mass and relative peak $\dot{V} \mathrm{O}_{2}$. Indeed, the gold standard for accounting for body size on peak $\dot{V} \mathrm{O}_{2}$ is to allometrically scale (Nevill et al., 2005), which has been missing from a large proportion of studies, including those specifically in asthma. When correcting for body size using allometric scaling, the higher peak $\dot{V} \mathrm{O}_{2}$ usually associated with increases in maturity are no longer different between maturational groups (Cunha et al., 2011, Cunha et al., 2016, McNarry et al., 2014b). The use of scaling peak $\dot{V} \mathrm{O}_{2}$ is of particular importance within asthma research due to the obese asthma phenotype. Whilst it is becoming common practice in asthma studies to account for body mass (Berntsen et al., 2009, van Veldhoven et al., 2001), to date no studies have used allometric scaling of peak $\dot{V} \mathrm{O}_{2}$.

The majority of our evidence with regard to cardiorespiratory fitness is based on a single measure, namely peak $\dot{V} \mathrm{O}_{2}$, but the use of one parameter of fitness does not appropriately represent the aerobic fitness of an individual and therefore should not be used exclusively. Moreover, even in those studies which have utilised peak $\dot{V} \mathrm{O}_{2}$ 
as a measure of cardiorespiratory fitness, the applicability of this measure to functional capacity during activities of daily living has been questioned (Jones, 2006, Matos \& Winsley, 2007). Indeed, differences between those with and without asthma may be detected using other parameters of aerobic fitness (Lucia et al., 2002). There is a lack of information regarding the influence of asthma on these other common factors of aerobic fitness considering the extensive use of incremental exercise tests. The use of submaximal parameters of aerobic function can provide a more comprehensive assessment of cardiorespiratory fitness; these include the lactate threshold or gas exchange threshold, the energy cost of exercise (termed $\dot{V} \mathrm{O}_{2}$ gain) or economy and the $\dot{V} \mathrm{O}_{2}$ kinetics. Whipp et al. (1982) outlines these parameters, inclusive of peak $\dot{V} \mathrm{O}_{2}$ as the four key parameters of aerobic fitness.

\subsubsection{Gas exchange threshold}

The lactate threshold is the metabolic rate at which the blood lactate concentration begins to rise above the baseline value. To determine the lactate threshold can be invasive and therefore surrogate measures have been devised. The Gas Exchange Threshold (GET, the break point at which $\dot{V} \mathrm{CO}_{2}$ production surpasses $\dot{V} \mathrm{O}_{2}$ ), ventilatory threshold (the point during exercise at which ventilation starts to increase at a faster rate than $\dot{V}_{2}$ ) and anaerobic threshold (the oxygen consumption above which aerobic energy production is supplemented by anaerobic mechanisms) all broadly reflect the lactate threshold, however, the GET will be taken to reflect the non-invasive equivalent of the lactate threshold. The GET which was first proposed by Beaver et al. (1986) evaluates changes in respiratory gas concentrations and is subsequently the respiratory gas equivalent of the lactate threshold. The GET is usually observed in adults between $45-60 \%$ of the peak $\dot{V} \mathrm{O}_{2}$ (Jones \& Poole, 2005) and between $45-70 \%$ peak $\dot{V} \mathrm{O}_{2}$ in children (Fawkner \& Armstrong, 2004a, Fawkner \& Armstrong, 2004b). The GET can be used to gain additional information about the fitness status of an individual as the earlier it occurs in relation to the peak $\dot{V} \mathrm{O}_{2}$, the poorer the fitness of the person. Indeed, it has been proposed that a GET of $<50 \%$ peak $\dot{V} \mathrm{O}_{2}$ is a marker of deconditioning (Urquhart \& Vendrusculo, 2017). The GET can also be used in the design and optimisation of training programmes specific to an individual. For example, training at the GET is potentially the optimal intensity for improving endurance fitness (Weltman et al., 1990, Mader, 1991), due to the lack of 
accumulation of blood lactate which may compromise training duration (Macdougall, 1977, Weltman, 1989). In addition, the exercise intensities associated with the GET are used to evaluate the $\dot{V} \mathrm{O}_{2}$ kinetics of a person allocating a specific intensity.

There is a paucity of data regarding the effect of asthma on GET in both children and adults; however, it has been used in previous studies to prescribe exercise intensities (Cochrane \& Clark, 1990, van Veldhoven et al., 2001, Varray et al., 1991). Furthermore, a study in adults with asthma found that the condition may affect $\dot{V} \mathrm{O}_{2 \max }$ whilst the ventilatory threshold was unaffected (Sirichana et al., 2014), similar to findings in adolescents with Cystic Fibrosis (Bongers et al., 2014). Previous studies in children with asthma found relatively normal GET (Ahmaidi et al., 1993, Counil et al., 2003, van Veldhoven et al., 2001, Varray et al., 1995, Varray et al., 1991), with only one showing possible deconditioning with a GET of $47.4 \%$ of peak $\dot{V} \mathrm{O}_{2}$ (Neder et al., 1999). The participants with possible deconditioning had moderate to severe asthma and therefore, severity of asthma may affect the GET. However, interpretation of these studies may be limited as they did not include a healthy control population. Only two studies assess the differences between healthy control and asthma participants, one in adults and one in children with equivocal results. The study in adults showed participants with asthma to have a lower anaerobic threshold than their healthy peers (Clark \& Cochrane, 1988), whilst the study in children showed no differences in anaerobic threshold between those with and without asthma (Santuz et al., 1997). One potential reason for the discrepancies between the two studies is that peak $\dot{V} \mathrm{O}_{2}$ between adults was shown to be reduced in comparison to their healthy peers, while the children's peak $\dot{V} \mathrm{O}_{2}$ was not different. However, whilst it may seem that peak $\dot{V} \mathrm{O}_{2}$ and GET are associated, it has previously been found that peak $\dot{V} \mathrm{O}_{2}$ can improve independently of any changes in GET (van Veldhoven et al., 2001). Therefore, previous studies finding differences in peak $\dot{V} \mathrm{O}_{2}$ following an exercise intervention may have missed other parameters of fitness remaining the same; which could aid in assessing whether the disease may cause derangements in the delivery or utilisation of $\mathrm{O}_{2}$. There is therefore a need to confirm the influence of asthma in adolescents on the GET and to confirm if an 
exercise programme influences the GET as observed in some (e.g., Ahmaidi et al., 1993), but not all studies (e.g., van Veldhoven et al., 2001).

\subsubsection{Gain}

The gain is a measure of aerobic exercise efficiency $\left(\mathrm{O}_{2}\right.$ cost of exercise) (Neder et al., 2001) and is measured by delta change $(\Delta)$ in $\dot{V} \mathrm{O}_{2}$ against work rate (WR) from an incremental ramp test to exhaustion $\left(\Delta \dot{V} \mathrm{O}_{2} / \Delta \mathrm{WR}\right)$. Abnormal muscle $\mathrm{O}_{2}$ delivery and utilisation can be assessed using gain (Gimenes et al., 2011, Hansen et al., 1987, Jones et al., 1998, Meyer et al., 1998, Neder et al., 2001) and a smaller gain denotes a reduced metabolic capacity to increase $\dot{V} \mathrm{O}_{2}$ in accord with increased work rate. The gain is usually observed to be about $\sim 10 \mathrm{ml} \cdot \mathrm{O}_{2} \cdot \mathrm{min}^{-1} \cdot \mathrm{W}^{-1}$. There are currently no studies examining the effect of asthma on gain and therefore it is not known if such derangements in the airways affect the delivery and utilisation of $\mathrm{O}_{2}$ in both children and adults. Previous studies into respiratory diseases such as Cystic Fibrosis have reported both a significantly smaller (Fielding et al., 2015) and no difference in the gain (Groen et al., 2010) compared to healthy counterparts. The former may suggest an impairment in oxygen transport and utilisation by the muscles, whereas the latter concludes diseases which are characterised by impaired oxygen delivery may not be as sensitive to small differences in the gain. Moreover, in adult COPD patients, gain was not significantly different from their healthy peers (Barron et al., 2016). Disparity between studies is likely due to the differences in disease severity of the participants but may also be due to methodological analysis of the $\Delta \dot{V} \mathrm{O}_{2} / \Delta \mathrm{WR}$ slope. When comparing previous studies it is important to only compare those which specify the section of the $\dot{V} \mathrm{O}_{2}$ WR response (e.g., Fielding et al., 2015) as $\dot{V} \mathrm{O}_{2}$ above the GET may not be linear in all participants and should be excluded from analysis (Boone \& Bourgois, 2012). Furthermore, if the whole response is analysed then it may also be skewed by the Mean Response Time (MRT, e.g., Groen et al. 2010) and therefore the initial minute of the test should be excluded from analysis (Barstow et al., 2000a).

\subsubsection{Mean response time}

The MRT is calculated as the point of intersection between the baseline $\dot{V} \mathrm{O}_{2}$ and a backwards linear extrapolation of the $\dot{V} \mathrm{O}_{2}$ by time slope from the onset of the 
increase in work rate (Glantz, 1990). The MRT of an incremental ramp test represents $\mathrm{O}_{2}$ utilisation by the muscle at the onset of exercise. The MRT is lacking from previous asthma studies and to the best of the authors knowledge has not been reported previously. This may be due to the MRT being a surrogate for $\dot{V} \mathrm{O}_{2}$ kinetics although these are also missing from the asthma literature. However, a previous study comparing disease differences in adolescents with Cystic Fibrosis found patients to have significantly longer MRT than their healthy peers (Fielding et al., 2015). This delay in oxygen uptake may relate to exercise intolerance as a longer MRT has been shown to lead to early fatigue (Poole et al., 2008). Furthermore, in a previous study the MRT was found to be inversely related to the $\mathrm{FEV}_{1} / \mathrm{FVC}$ ratio (Fielding et al., 2015), which indicates that participants with asthma, especially those with greater airway obstruction, may have a slower MRT due to an impairment in $\mathrm{O}_{2}$ delivery. It is therefore necessary to evaluate the differences in MRT between adolescents with and without asthma to evaluate their exercise tolerance as it may explain possible differences in fitness.

\subsubsection{Oxygen uptake kinetics}

Although maximal incremental tests are useful for assessing aerobic fitness, as previously stated, the applicability to daily life is questionable as it is rare that a person would exercise as their maximal aerobic capacity (Jones \& Poole, 2005). In addition, peak $\dot{V} \mathrm{O}_{2}$ does not assess changes in metabolic demand during day-to-day activities. Indeed, assessing $\dot{V} \mathrm{O}_{2}$ kinetics which reflect the dynamic $\dot{V} \mathrm{O}_{2}$ response to an instantaneous change in the metabolic demand (Grassi et al., 1996, Krustrup et al., 2009) may better assess daily activities. The onset of exercise provides a noninvasive window into the metabolic activity of the muscle (Armstrong \& Barker, 2009), and assesses, ultimately, the ability to tolerate physical activity (Jones \& Poole, 2005). Constant work rate tests set to submaximal work rates are best used to

assess the $\dot{V} \mathrm{O}_{2}$ kinetic response to exercise. $\dot{V} \mathrm{O}_{2}$ kinetics following constant work rate exercise can assess the integrated capacity of the body to transport and utilise $\mathrm{O}_{2}$ to supply the increased rate of energy turnover in the contracting myocytes (Whipp \& Ward, 1990). 
The $\dot{V} \mathrm{O}_{2}$ kinetic response to onset of constant work rate exercise is characterised by a three-phase pulmonary response (Whipp \& Wasserman, 1972). Phase I, termed the cardiodynamic phase, consists of a rapid increase in $\dot{V} \mathrm{O}_{2}$ which is usually initiated at the first breath (Jones \& Poole, 2005). The cardiodynamic phase typically occurs in children within the first 15 seconds of the exercise onset (Hebestreit et al., 1998, Springer et al., 1991, Fawkner et al., 2002). During the cardiodynamic phase there is a dissociation between muscle and pulmonary $\dot{V} \mathrm{O}_{2}$ due to the muscle-lung transit delay. Moreover, the cardiodynamic phase is only observed in the pulmonary $\dot{V} \mathrm{O}_{2}$ response, whereas the muscle $\dot{V} \mathrm{O}_{2}$ increases in a mono-exponential fashion from the onset of exercise. If phase I is appropriately accounted for, then the time (represented by the time constant) and amplitude (both primary and slow component) will be in close agreement for both the muscle and pulmonary $\dot{V} \mathrm{O}_{2}$ responses (within $\pm 10 \%$, Grassi et al., 1996, Krustrup et al., 2009). Subsequent to the cardiodynamic phase, the pulmonary $\dot{V} \mathrm{O}_{2}$ increases exponentially to a steady state, this is known as phase II. The speed of the exponential increase of the $\dot{V} \mathrm{O}_{2}$ during phase II is described by the time constant, which reflects the time to achieve $63 \%$ of the total. The phase II $\dot{V} \mathrm{O}_{2}$ time constant is approximately 20-30 seconds in children during cycling (Breese et al., 2012, Koga et al., 1999). During moderate-intensity (exercise below the GET) phase II is followed by phase III which is the attainment of a steady state $\dot{V} \mathrm{O}_{2}$ where the $\mathrm{O}_{2}$ demand is matched with $\mathrm{O}_{2}$ utilisation. Phase III is achieved in moderateintensity exercise after approximately 2-3 minutes (Jones \& Poole, 2005).

Heavy intensity constant work rate exercise utilises work rates above the GET but below critical power, which demarcates the boundary where energy provision is wholly oxidative. Caution should be taken when assessing the intensity domains in children as they are characterised by a significantly smaller peak $\dot{V} \mathrm{O}_{2}$ (Armstrong \& Barker, 2009). There is therefore only a small range of work rates within a given exercise intensity domain, especially within heavy-intensity as the GET typically occurs between 55-60\% peak $\dot{\mathrm{V}} \mathrm{O}_{2}$ (Fawkner \& Armstrong, 2002a, Pringle et al., $2003)$ with critical power occurring at approximately $70-82 \%$ peak $\dot{V} \mathrm{O}_{2}($ Fawkner \& Armstrong, 2002b). During heavy intensity exercise, the dynamic $\dot{V} \mathrm{O}_{2}$ response becomes more complex, with the addition of a "slow component" (Barstow \& Mole, 1991, Whipp \& Wasserman, 1972), that originates principally within the exercising 
muscles (Poole \& Jones, 2012). The slow component occurs during heavy intensity exercise after 90-150 seconds (Barstow \& Mole, 1991, Paterson \& Whipp, 1991) and usually delays the attainment of steady state until 10-15 minutes dependant on exercise intensity or fitness of the participant. Typically in children a slow component is also evident, albeit at a reduced amplitude in comparison to adults (Obert et al., 2000, Fawkner \& Armstrong, 2003a). Exercise above the critical power engenders a $\dot{V} \mathrm{O}_{2}$ slow-component which elevates to $\dot{V} \mathrm{O}_{2 \max }$, therefore demonstrating that the $\dot{V} \mathrm{O}_{2}$ slow component is related to muscle fatigue and exercise tolerance.

The $\dot{V} \mathrm{O}_{2}$ kinetic response has previously been assessed in differing populations and has been shown to be highly sensitive to both advantageous and deleterious influences in adults, for example, training and diseases, respectfully. As such, the dynamic $\dot{V} \mathrm{O}_{2}$ response may be displaced by disease (Poole \& Jones, 2012). Previous research into the effects of other pulmonary diseases showed impaired $\dot{V} \mathrm{O}_{2}$ kinetics in comparison to age-matched healthy controls in those with Emphysema and Idiopathic Pulmonary Fibrosis (McNarry et al., 2017), Cystic Fibrosis (Saynor et al., 2016) and Chronic Obstructive Pulmonary Disease (Nery et al., 1982, PuenteMaestu et al., 2000). However, there are currently no studies which have assessed the effect of asthma on $\dot{V} \mathrm{O}_{2}$ kinetics. Consequently, due to participants with other respiratory diseases having diminished $\dot{V} \mathrm{O}_{2}$ kinetics, which may be attributable to an impaired $\mathrm{O}_{2}$ delivery through a low arterial $\mathrm{O}_{2}$ content caused by mismatched ventilation and gas exchange in the lungs (Jones \& Poole, 2005), there is a requirement to assess if there is an effect of asthma on $\dot{V} \mathrm{O}_{2}$ kinetics. If the $\dot{V} \mathrm{O}_{2}$ kinetics are found to differ between those with and without asthma, there is a greater chance of the $\mathrm{O}_{2}$ delivery being affected due to the inflammation of the airways in adolescents with asthma. Therefore, if a difference is found in $\dot{V} \mathrm{O}_{2}$ kinetics between those with and without asthma, the time constant and the MRT are likely to be slowed in the adolescents with asthma. Indeed, as improved $\dot{V} \mathrm{O}_{2}$ kinetics would be expected to reduce fatigue development it may therefore aid in assessing improvements in exercise tolerance, which has previously been shown to reduce asthma symptoms (Milgrom \& Taussig, 1999). Moreover, in addition to a potential influence of asthma on $\dot{V} \mathrm{O}_{2}$ kinetics, due to impaired lung function, adaptations to exercise may differ from healthy peers. 
The determinants of $\dot{V} \mathrm{O}_{2}$ kinetic response are still a contentious issue, with muscle $\mathrm{O}_{2}$ delivery and $\mathrm{O}_{2}$ utilisation proposed to be rate limiting (Poole et al., 2008). Oxygen delivery may be limited at a point during the transport of $\mathrm{O}_{2}$ from inhalation to the mitochondria which may affect the speed of $\dot{V} \mathrm{O}_{2}$ kinetics (Poole et al., 2008). In addition, $\dot{V} \mathrm{O}_{2}$ kinetics may also be affected by the utilisation or extraction of $\mathrm{O}_{2}$ denoted by an intrinsic slowness of intracellular oxidative metabolism when adapting to the change in metabolic demand (Payne et al., 1997). $\dot{V} \mathrm{O}_{2}$ kinetics are likely effected by both $\mathrm{O}_{2}$ delivery and utilisation, however, in previous work, the most important factor for the slowing of $\dot{V} \mathrm{O}_{2}$ kinetics is debated between $\mathrm{O}_{2}$ delivery (Krustrup et al., 2004) and $\mathrm{O}_{2}$ utilisation (Bailey et al., 2009). Therefore, both $\mathrm{O}_{2}$ delivery (central) and utilisation (peripheral function) should be measured simultaneously to assess whether adolescents with asthma can match $\mathrm{O}_{2}$ delivery to $\mathrm{O}_{2}$ utilisation during constant work rate exercise. Additionally, these measures can evaluate the mechanistic bases for the effect of asthma, if any, on the $\dot{V} \mathrm{O}_{2}$ kinetic response and subsequent adaptation to an exercise intervention.

More recent methods for providing information on $\mathrm{O}_{2}$ extraction and delivery include near-infrared spectroscopy (NIRS) and HR kinetics, respectively. The use of NIRS can estimate the fractional $\mathrm{O}_{2}$ extraction within a localised area of the microcirculation through measuring the relative change in the concentration of oxygenated and deoxygenated haemoglobin and myoglobin ([HHb], Boushel et al., 2001). The balance between $\mathrm{O}_{2}$ delivery and utilisation is indicated by the [HHb] signal which then provides the index of fractional $\mathrm{O}_{2}$ extraction (DeLorey et al., 2007, Grassi et al., 2003). NIRS may therefore be useful for understanding alterations caused by changes in aerobic fitness and muscle oxidative metabolism in adolescents with asthma. However, NIRS is unable to distinguish between $\mathrm{O}_{2}$ delivery and utilisation and therefore conclusions can only be drawn about the balance between the two. The HR kinetics reflect cardiac output kinetics (Miyamoto et al., 1982, Yoshida \& Whipp, 1994) and is a crude estimate of muscle blood flow and estimating bulk oxygen delivery (MacPhee et al., 2005), but may provide further insight into the mechanisms behind $\dot{V} \mathrm{O}_{2}$ kinetics and how they are altered by training (Bailey et al., 2009). Previously, techniques to investigate changes in $\mathrm{O}_{2}$ delivery and muscle blood flow were costly, invasive and not ethically sound for use in paediatric 
populations, for example muscle biopsy, tissue $\mathrm{O}_{2}$ microelectrodes and myoglobin $\mathrm{O}_{2}$ saturation by spectrophotometric analysis. NIRS is used in recent research (McNarry et al., 2015a, McNarry et al., 2017) and the current thesis as it is relatively cheap, portable, non-invasive and most importantly provides a continuous measure to provide measures of the dynamic changes in tissue oxygenation.

\subsubsection{Training effects of $\dot{V} \mathrm{O}_{2}$ kinetics}

Previously, in adults, a faster $\dot{V} \mathrm{O}_{2}$ time constant was observed as a result of exercise training (Murias et al., 2010, Raleigh et al., 2016), however, less is known about the $\dot{V} \mathrm{O}_{2}$ kinetic response in youth, with limited longitudinal studies and contradictory results in trained vs. untrained children and adolescents. Indeed, in pre-pubertal children, Winlove et al. (2010) reported a faster $\dot{V} \mathrm{O}_{2}$ kinetic time constant in trained swimmers in comparison to their untrained peers, whilst in contrast, two studies reported no difference between trained and untrained pre-pubertal children (Cleuziou et al., 2002, Obert et al., 2000). Moreover, in adolescents, training was associated with a faster $\dot{V} \mathrm{O}_{2}$ kinetic time constant than their untrained peers (Marwood et al., 2010, McNarry et al., 2011c, Unnithan et al., 2015). The conflicting results may be explained by different modalities between training and experimental measures. However, training status is likely to explain the differences associated with differing modalities as the influence of training in pre-pubertal swimmers were only manifest during upper body ergometry (Winlove et al., 2010), which may be due to the sport as it is predominately upper-body (Ogita et al., 1996). Furthermore, cross-sectional studies cannot be used to assess whether the faster $\dot{V} \mathrm{O}_{2}$ kinetics observed in trained individuals are a result of long-term training per se or the product of genetic selfselection. Therefore, there is a need to investigate whether training interventions can significantly improve $\dot{V} \mathrm{O}_{2}$ kinetics in previously untrained children and adolescents. Indeed, to date, only one longitudinal study in pre-pubertal children assessing the effect of exercise on the $\dot{V} \mathrm{O}_{2}$ kinetic response has been conducted (McNarry et al., 2015b). The study utilised high-intensity interval training which engendered a faster $\dot{V} \mathrm{O}_{2}$ kinetic time constant in obese children but did not have an effect on non-obese participants. Interestingly, peak $\dot{V} \mathrm{O}_{2}$ was unaffected by the intervention, which further justifies the use of $\dot{V} \mathrm{O}_{2}$ kinetics analysis within asthma studies to assess if quality of life is associated with improved $\dot{V} \mathrm{O}_{2}$ kinetics irrespective of improved 
peak $\dot{V} \mathrm{O}_{2}$. Differences in $\dot{V} \mathrm{O}_{2}$ kinetics between participants in the study of McNarry et al. (2015b) could have been due to higher baseline fitness in normal-weight children and therefore a longer study may be required to engender a significant improvement. Furthermore, the differences shown could have been a result of methodological shortcomings as multiple transitions were not utilised. Therefore, there is a gap in the literature on the effect of an exercise intervention on $\dot{V} \mathrm{O}_{2}$ kinetics of adolescents, in addition to the effects of asthma.

Previously $[\mathrm{HHb}]$ and HR kinetics have been used to assess the differences between trained and untrained adolescents in soccer and swimming (Marwood et al., 2010, McNarry et al., 2011c, Unnithan et al., 2015). The two studies in soccer compared responses to moderate-intensity exercise in trained and untrained participants and found the trained participants to have a significantly faster $\dot{V} \mathrm{O}_{2}$ kinetic response (Marwood et al., 2010, Unnithan et al., 2015). The [HHb] response was comparable in trained and untrained boys, however, the HR kinetic response was faster in trained participants, which was attributed to increased $\mathrm{O}_{2}$ delivery and utilisation. Trained girls, however, exhibited a faster $[\mathrm{HHb}]$ response in comparison to their peers and although no estimate of $\mathrm{O}_{2}$ delivery was used, the authors speculated the faster $\dot{V} \mathrm{O}_{2}$ kinetics in trained girls was from an enhanced $\mathrm{O}_{2}$ utilisation in the muscle. The conflicting results suggest there is a possible sexual dimorphism within trained adolescents with regard to the $\dot{V} \mathrm{O}_{2}$ kinetic response. McNarry et al. (2011c) also found a faster $\dot{V} \mathrm{O}_{2}$ kinetic response to heavy-intensity exercise in adolescent swimmers. In agreement with Unnithan et al. (2015) and conflicting Marwood et al. (2010), faster HR and [HHb] kinetics were observed in trained participants suggests the faster $\dot{V} \mathrm{O}_{2}$ kinetic response was a function of enhanced $\mathrm{O}_{2}$ delivery and utilisation. Without a confirmation of the peripheral and central adaptations in boys, a conclusion on the sexual dimorphism is precluded. However, a faster $\dot{V} \mathrm{O}_{2}$ kinetic response to exercise in trained adolescents is likely to be due to a function of both faster $\mathrm{O}_{2}$ delivery and greater $\mathrm{O}_{2}$ extraction as observed in adults (McKay et al., 2009, Poole et al., 2008). In addition, although cross-sectional studies provide an informative comparison of training effects, they are unable to distinguish between genetic selection and an actual training effect. Therefore, further research is required to ascertain the $\mathrm{O}_{2}$ delivery and $\mathrm{O}_{2}$ utilisation response to an exercise intervention. 
Finally, within the asthma adolescent population, in addition to the pulmonary $\dot{V} \mathrm{O}_{2}$ kinetic response to constant work rate exercise, the $\mathrm{O}_{2}$ delivery and utilisation during constant work rate exercise has yet to be established. Therefore, further work is required to evaluate the effect of asthma and subsequently the effect of an exercise intervention on the $\dot{V} \mathrm{O}_{2}$ kinetic response and the mechanisms behind said response.

\subsection{Exercise interventions in asthma}

Early studies of exercise interventions for adults with asthma, found significant improvements in $\dot{V} \mathrm{O}_{2 \max }$ (Cochrane \& Clark, 1990), specifically, Haas et al. (1987) found a $15 \%$ increase in $\dot{V} \mathrm{O}_{2 \max }$. Furthermore, according to two reviews on exercise conditioning in asthma almost all studies showed improvements in both cardiovascular fitness and exercise performance and none showed a decrease in fitness (Eichenberger et al., 2013, Ram et al., 2000). These findings are not surprising considering that in healthy individuals, physical training has been shown to improve maximal aerobic fitness by up to $20 \%$ (Milanovic et al., 2015).

The effect of exercise training on pulmonary function in adults with asthma is debated, with some studies showing significant improvements in $\mathrm{FEV}_{1}$ and peak expiratory flow (PEF, Farid et al., 2005, Heba \& Ashraf, 2013), although the majority reported no change (Cochrane \& Clark, 1990, Lucas \& Platts-Mills, 2005). Conversely, although lung function did not improve in some studies as measured by $\mathrm{FEV}_{1}$, Mendes et al. (2010) found improvements in asthma free days and also found exercise to reduce the number of asthma exacerbations.

These studies have been reinforced by more recent intervention studies and are summarised by a meta-analysis on physical training for asthma by Carson et al. (2013) utilising the results of 21 studies. The pooled data showed exercise training significantly improved cardiovascular fitness through an increase in $\dot{V} \mathrm{O}_{2 \max }$, maximal expiratory volume, and heart rate maximum (Carson et al., 2013). Improvements in heart rate maximum may be due to the participants not reaching their maximal effort at baseline, which may question the results of the improvements in $\dot{V} \mathrm{O}_{2 \max }$. However, an alternative potential reason for the improvements in 
maximal heart rate is an improvement in cardiorespiratory fitness which allowed those who were perhaps deconditioned at baseline to continue the test for a longer duration.

There were, however, no significant changes in lung function, specifically PEF, $\mathrm{FEV}_{1}$ and $\mathrm{FVC}$, this is to be expected considering there is no obvious reason for improved PEF or $\mathrm{FEV}_{1}$ as a result of exercise training (Ram et al., 2000). Both quality of life and asthma symptoms were unable to be pooled due to the heterogeneity of the scales and instruments used, respectively. Asthma symptoms findings were equivocal but there was a trend for more to not report any significant changes during the intervention (e.g., Boyd et al., 2012, Swann \& Hanson, 1983, Turner et al., 2010). However, in a previous systematic review, several factors were found to contribute to a possible increase in asthma related quality of life directly related to asthma symptoms, such as days per month free of asthma symptoms and a reduction in asthma medication (Eichenberger et al., 2013). Moreover, quality of life was found to increase in the review by Carson et al. (2013) in 4 out of 5 of the studies (Fanelli et al., 2007, Goncalves et al., 2008, Mendes et al., 2010, Turner et al., 2010) and therefore this suggests that exercise interventions may be used to increase quality of life in asthma populations. This would seem reasonable considering that quality of life is associated with participants' dependence on asthma medication (van den Bemt et al., 2010). Furthermore, none of the studies reported in either review found a worsening of asthma symptoms following physical training and therefore it should be considered safe within asthma populations.

Exercise interventions in children and adolescents with asthma have resulted in similar findings to that of adults with regard to increased cardiorespiratory fitness and exercise tolerance and unchanged pulmonary function. A recent review by Wanrooij et al. (2014) evaluated the effects of exercise training in children and adolescents with asthma. From the 5987 studies identified, only 29 met the inclusion criteria with a total of 1045 children with asthma (Wanrooij et al., 2014), demonstrating the paucity of research regarding exercise in children with asthma. However, these exercise studies in children do show some promising results in eliciting advantageous adaptations in maximal aerobic capacity, EIB and quality of life. 
Lung function parameters are one of the more common measurements for assessing training interventions with asthma; of the 24 studies identified by Wanrooij et al. (2014), few resulted in significant improvements. However, three exercise interventions did elicit significant improvements in $\mathrm{FEV}_{1}(6-12 \%)$ in participants (Chang et al., 2008, Onur et al., 2011, Sidiropoulou et al., 2007). Moreover, FVC was only found to significantly increase (10-14\%) in 2 studies (Chang et al., 2008, Onur et al., 2011). These results were challenged by the authors, with one suggesting the change in results whilst significant did not represent a meaningful change, while the other studies were unable to elucidate the mechanisms. Exercise was found to elicit improvements in PEF, albeit mildly (29-40\%), in 4 out of 9 studies (Chang et al., 2008, Counil et al., 2003, Lima et al., 2008, Wang \& Hung, 2009). The slight increase in PEF suggests increased strength of the intercostal muscles, the diaphragm and abdominals (used for exhaling and compressing the diaphragm upwards into the chest cavity) post training, which is may be confirmed by the lack of increase in $\mathrm{FEV}_{1}$ following training (Wanrooij et al., 2014). These increases in strength could be a result of increased maturation throughout the studies. Another possible factor for the improvements could be a reduced BMI following the intervention as lung function can be compromised by obesity (Lessard et al., 2008), which is an important point considering the obese asthma phenotype. In a recent exercise intervention the authors highlight the importance of a weight reduction (5\% body fat) in their intervention participants which led to an improvement in lung function (FEV1 increase of 1.22l) and subsequently quality of life (60\% increase, Latorre-Roman et al., 2014). Moreover, PEF variability has previously been reported to be highly variable and therefore the increase may not be a result of the exercise intervention (Global Initiative for Asthma, 2017). No other differences were found following exercise training in other parameters of lung function, which is understandable considering the lack of reason for increased exercise alone to result in improvements in lung function (Ram et al., 2000). These findings suggest that exercise training generally does not improve lung function in children with asthma (Wanrooij et al., 2014).

Evidence of an effect of physical training on children's asthma control and quality of life is lacking with contradictory results reported. Specifically, Basaran et al. (2006) 
and Fanelli et al. (2007) found exercise training to improve quality of life in children with asthma but Moreira et al. (2008) did not. This discrepancy between studies may be due to a higher baseline quality of life in participants in the study by Moreira et al. (2008), as there may be a ceiling effect whereby quality of life cannot be further improved by exercise. With regards to the effect of exercise training on asthma control, the limited number of studies suggest a decrease in number of attacks and days of wheezing (Huang et al., 1989, Lima et al., 2008, Sly et al., 1972). In addition, symptoms scores improved in one study (Lima et al., 2008), however in contrast, exercise did not result in medication score reduction (Basaran et al., 2006, King et al., 1989, Neder et al., 1999).

One purpose of implementing an exercise intervention is to improve cardiorespiratory fitness. In a systematic review by Strong et al. (2005), 18 correlational studies were found to suggest a positive relationship between physical activity and maximal and submaximal cardiorespiratory fitness. This is also confirmed in children with asthma who demonstrate maximal oxygen uptake to positively correlate with physical activity (Garfinkel at al., 1992). Seventeen of the studies evaluated by Wanrooij et al. (2014) examined the effects of exercise on aerobic capacity, 10 of which assessed peak $\dot{V} \mathrm{O}_{2}$. Significant improvements in peak $\dot{V} \mathrm{O}_{2}$ were observed post exercise training intervention in children with asthma (Ahmaidi et al., 1993, Counil et al., 2003, van Veldhoven et al., 2001, Varray et al., 1995, Varray et al., 1991), however, few of these took physical activity into consideration. This was, however, not the case for all studies as several reported no significant increases in peak $\dot{V} \mathrm{O}_{2}$ following exercise training (Fitch et al., 1986, Graff-Lonnevig et al., 1980, Holzer et al., 1984, King et al., 1989, Neder et al., 1999). Reasons for the lack of increase range from short interventions (<3 weeks), using low-intensity training and type of exercise vs. modality of measure. All studies differed with regard to type of training, frequency, duration, intensity and the length of training programme and therefore further work is required to design an intervention which would elicit the greatest adaptations; as discussed below. In summary, given the benefits of exercise to general health, exercise may be used as an effective means of improving asthma-related quality of life, symptoms and cardiorespiratory fitness in adolescents with asthma. 


\subsection{Exercise intervention design}

When implementing an exercise intervention, the mode, intensity, frequency and duration need to be considered (Bouchard et al., 2012). The majority of the interventions previously discussed in this review do not explicitly state the intensity of their exercise (Basaran et al., 2006, Graff-Lonnevig et al., 1980, van Veldhoven et al., 2001), however, they are mostly within the moderate-intensity range. Constant intensity exercise training (CIET), which is defined as any exercise using large muscle groups that is performed continuously in a rhythmic manner and is typically conducted at $70-75 \%$ heart rate maximum (Foxdal et al., 1996), best describes the previous studies. In a recent systematic review of 21 training studies in non-asthma youth, almost all studies evaluated CIET, assessing peak $\dot{V} \mathrm{O}_{2}$ (Armstrong \& Barker, 2011). There was also a large variation in frequency of training, duration and intensity. A review in training and aerobic fitness in healthy young people showed studies utilising training at or above the GET resulted in significant improvements in peak $\dot{V} \mathrm{O}_{2}$ (Baquet et al., 2003). Moreover, the studies which failed to improve peak $\dot{V} \mathrm{O}_{2}$ utilised intensities between $70-80 \%$ heart rate maximum. In addition, although previous exercise interventions in children and adolescents with asthma have mostly been in CIET, in a recent systematic review (Wanrooij, et al., 2014), exercise intensity at or above the GET for 60 minutes or more resulted in more favourable increases in aerobic fitness (Ahmaidi et al., 1993, Counil et al., 2003, Varray et al., 1995, Varray et al., 1991). Moreover, exercise interventions at the GET but for shorter periods of time or at a lower intensity were less likely to elicit positive adaptations in aerobic fitness (Fitch et al., 1986, Graff-Lonnevig et al., 1980, Holzer et al., 1984, Neder et al., 1999). Therefore, as duration and intensity is yet to be agreed within the literature and a higher intensity would seem to elicit more favourable adaptations, future interventions should explore the use of high intensity training. In addition to training at higher intensities, the systematic review by Wanrooij et al. (2014) suggested that exercise interventions were more likely to elicit positive improvements in peak $\dot{V} \mathrm{O}_{2}$ if the duration of the sessions were for at least 120 minutes per week divided over at least two and were in excess of 3 months (Ahmaidi et al., 1993, Counil et al., 2003, van Veldhoven et al., 2001, Varray et al., 1995, Varray et al., 1991). 
Moderate-intensity continuous exercise training can be monotonous, which is synonymous with study drop out (McNarry et al., 2015b), time consuming and may not be appealing to adolescent populations (Buchan et al., 2011). In contrast to CIET, high-intensity interval training (HIIT), also known as high-intensity intermittent exercise (HIIE), consists of repeated, intense, short bouts of exercise interspersed with either rest of or active recovery (Buchheit \& Laursen, 2013, Laursen \& Jenkins, 2002). The short durations of near maximal exercise usually last between a few seconds to several minutes (Gibala \& McGee, 2008). The rest or low intensity recovery is often equal that of the exercise bout (Ross et al., 2016). Rate of perceived exertion, maximal aerobic speed, heart rate and $\dot{V} \mathrm{O}_{2}$ can be used to assess exercise intensity during exercise. For HIIT, the exercise bouts are required to be greater than or equal to $90 \%$ peak $\dot{V} \mathrm{O}_{2}, 100 \%$ maximal aerobic speed, and/or $90 \%$ heart rate maximum (Baker, 2011, Gibala \& McGee, 2008, Hood et al., 2011, Little et al., 2011).

The use of HIIT is currently very promising as an alternative to traditional CIET programmes, however the evidence is limited in youth, especially the optimisation of HIIT programmes and the physiological mechanisms responsible for training induced changes (McNarry \& Armstong, 2017). As youths heart rates can recover more rapidly and are able to maintain a high work rate interspersed with short rest periods for a greater number than adults (Ratel \& Williams, 2017), the lack of HIIT protocol training studies in children and adolescents is surprising. Considering that HIIT and CIET were shown to be able to increase peak $\dot{V} \mathrm{O}_{2}$ in youth over 20 years ago (McManus et al., 1997), the use of HIIT has only recently gained focus. Costigan et al. (2015) conducted a systematic review and meta-analysis and concluded that HIIT is effective at increasing peak $\dot{V} \mathrm{O}_{2}$ in adolescents. Indeed, a meta-analysis by Weston et al. (2014a) reported HIIT to elicit a greater increase in cardiorespiratory fitness in comparison to a moderate-intensity intervention. Perceived lack of time has commonly been cited as a barrier to exercise (Dwyer et al., 2006, Lovell et al., 2010, Reichert et al., 2007, Welch et al., 2009). Furthermore, HIIT has been suggested to be a time-efficient method of exercise that can elicit significant improvements in peak $\dot{V} \mathrm{O}_{2}$ as CIET in youth (Gibala et al., 2012, Kessler et al., 2012, Warburton et al., 2005, Weston et al., 2014b). With regard to asthma, it 
has been concluded that relatively brief periods of high-intensity exercise are at a low risk for asthma symptoms (Del Giacco et al., 2015). Indeed, continuous exercise has been suggested to provoke and trigger asthma symptoms (Sidiropoulou et al., 2007), as such, intermittent exercise may not provoke asthma and might aid in improving adolescents' tolerance of exercise, increasing endurance (Del Giacco et al., 2015), highlighting the potential utility of HIIT. The intermittent nature of HIIT may also facilitate a decrease in end expiratory lung volume during the resting phase (Beauchamp et al., 2010), reducing the risk of an asthma attack. The use of HIIT appears to be safe in adolescents with asthma (Latorre-Roman et al., 2014) and should therefore be considered within asthma studies.

Not only has HIIT been shown to elicit greater improvements in cardiorespiratory fitness, it is also perceived in adolescents to be more enjoyable to participate in in comparison to CIET (Malik et al., 2017). Specifically, O’Neil and Dogra (2017) found interval exercise was associated with lower ratings of perceived exertion and dyspnoea in comparison to constant-intensity exercise in adults with asthma. These lower ratings are likely due to the rest periods included within the training. In children, enjoyment was also highlighted as essential to increasing effort required for reaching high intensities (Westergren et al., 2016).

When utilising a HIIT intervention, safety must be considered. Indeed, concerns such as risk of injury in previously sedentary, overweight or clinical populations were perceived as barriers to HIIT (Donahue et al., 2006). However, in recent reviews, the beneficial effects of HIIT have been shown in clinical populations (Guiraud et al., 2012, Hwang et al., 2011, Kessler et al., 2012, Ross et al., 2016, Weston et al., 2014a). With regard to asthma, in all studies utilising HIIT no asthma attacks were shown to be caused by the exercise protocols and the participants tolerated the exercise well (Counil et al., 2003, Latorre-Roman et al., 2014, Varray et al., 1991).

The use of HIIT in asthma studies is lacking. The first study which utilised HIIT as an exercise intervention in children with asthma (Varray et al., 1991) did so immediately following 3 months of CIET. The study showed significant increases in peak $\dot{V} \mathrm{O}_{2}$ in children with asthma following CIET but no such improvements during the following 3 months of HIIT. These findings are somewhat surprising due to 
studies in healthy populations eliciting increases in peak $\dot{V} \mathrm{O}_{2}$ following HIIT (Costigan et al., 2015). Due to the prior CIET intervention, which elicited significant improvements in peak $\dot{V} \mathrm{O}_{2}$, the baseline fitness is increased which may confound the interpretation of the secondary HIIT intervention. Indeed, the increases in baseline peak $\dot{V} \mathrm{O}_{2}$ may result in a ceiling effect as baseline fitness is known to influence the magnitude of change elicited by an intervention in youth (Eliakim et al., 1996, Mandigout et al., 2001, Tolfrey et al., 1998). Furthermore, the intervention may not have elicited changes in peak $\dot{V} \mathrm{O}_{2}$ due to the short durations of exercise in comparison to rest period; therefore far less time was spent exercising in comparison to their prior CIET intervention.

Two studies on HIIT in adults followed with the same primary author (Emtner, 1996, Emtner et al., 1998). Both studies consisted of a HIIT intervention, the first in water and the second on land, which elicited significant improvements in lung function and cardiorespiratory fitness, accompanied by a reduction in anxiety towards exercise. However, although the exercise intensity was stated to be high-intensity, caution should be taken when interpreting findings as their target work rate was between 8090\% heart rate maximum, which does not conform to modern HIIT training interventions of $>90 \%$ heart rate maximum (Hood et al., 2011, Little et al., 2011).

Counil et al. (2003) utilised a higher intensity work rate, opting for sprints at maximal aerobic power as a HIIT intervention in children with asthma which showed significant increases in peak $\dot{V} \mathrm{O}_{2}$. The sessions conducted were well tolerated, however, instead of rest periods, Counil et al. (2003) utilised exercise at the GET; the continuous aspect of this protocol may put asthma participants at a higher risk of asthma symptoms (Del Giacco et al., 2015).

More recently, a study conducted in children by Latorre-Roman et al. (2014) utilised a combination of HIIT and CIET which resulted in an increased in lung function, fitness and asthma-related quality of life. The intervention of 60 minutes of exercise 3 times per week for 12 weeks, consisted of 40 minutes of exercise, however, heart rate monitors were not worn and therefore the true intensity of the sessions cannot be discussed. 
Finally in the most recent HIIT intervention in adult participants with asthma (Toennesen et al., 2018) elicited significant improvements in $\dot{V} \mathrm{O}_{2 \max }$ with no improvements in asthma control or lung function. The study was limited by the lack of a control group and the methodological training intensities which may hinder the use of the intervention in a wider group.

\subsubsection{Work:rest ratio}

Previous HIIT interventions in participants with asthma vary methodologically with regards to both modality and the work:rest ratio. Varray et al. (1991) utilised a 1minute intervals which included both the exercise $(25 \mathrm{~m}$ swim) and rest. This method may hinder results as each participant may have been able to complete the exercise in a different length of time giving each person a different work:rest ratio. Emtner et al. (1996 \& 1998) used a work:rest ratio of 2-minute sprints interspersed by 1.5 minutes rest. This method was tested both on land and in water with similar results, the use of a shorter rest period to exercise may have aided in improvements in peak $\dot{V} \mathrm{O}_{2}$ (Kavaliauskas et al., 2015). Toennesen et al. (2018) utilised two to four five consecutive 1-minute intervals consisting of 30, 20, and 10 seconds at $<30,<60$ and $>90 \%$ HRmax, respectively, interspersed with 2-minute recovery periods (5:2, work:rest). Whilst this protocol utilised high-intensity exercise, it was very short totalling between 100-200 seconds per session interspersed with both rest and lowerintensity exercise possibly targeting endurance over improvements in peak $\dot{V} \mathrm{O}_{2}$.

Two other HIIT interventions interspersed their high-intensity work periods with active rest; Counil et al., (2003) had their participants exercise at the GET, for their active rest, over a period of 4 minutes (1:4, work:active-rest), and Latorre-Roman et al. (2014) at a "low-intensity" (unspecified ratio). Whilst these methods were shown to improve cardiorespiratory fitness and may also have greater endurance-based gains than protocols with passive rest periods (Milanović et al., 2015), these studies are effectively continuous exercise studies which may pose a risk to the participants with asthma, increasing the likelihood of asthma symptoms (del Giacco et al., 2015).

Whilst work:rest ratios have been suggested to be $\leq 1$ for the greatest improvements in cardiorespiratory fitness (Weston et al., 2014), only one study has been conducted in participants with asthma which utilised HIIT with a 1:1 work:rest ratio (de Silva et 
al., 2016) and this was performed in adults. The study found HIIT to improve cardiorespiratory fitness in a similar manner to continuous exercise but to have a greater effect on asthma control. The use of HIIT in participants with asthma has clear merits, however, has been hindered by mixed design studies. As such, no pure 1:1 work:rest ratio HIIT intervention has been published to date in adolescents with asthma and therefore the efficacy of HIIT is currently unknown.

\subsection{Conclusion}

In conclusion, there are equivocal findings in adolescents with asthma in comparison to their healthy peers with regards to physical activity, cardiorespiratory fitness and BMI. In addition, there are no previous studies into the effect of asthma on the submaximal aerobic fitness of adolescents. Therefore, further evidence is required to add to the existing body of research to evaluate the effect of, and interaction with, asthma.

Due to the personal and financial burden associated with asthma, finding a nonpharmacological, cost-effective way to reduce symptoms and improve asthma control that is also accessible is required. One such suggestion is exercise and whilst it is a common trigger for asthma symptoms, it has been found to improve fitness, and subsequently quality of life, asthma control and, in some cases, lung function. Previous studies have mostly utilised moderate-intensity exercise interventions, however, higher intensity exercise interventions were shown to elicit greater improvements in cardiorespiratory fitness. Moreover, intermittent exercise should be utilised in future studies as it has less risk of exacerbating asthma symptoms in comparison to continuous training. In addition, HIIT has been shown to improve cardiorespiratory fitness and BMI in healthy participants. However, despite widespread interest in HIIT, little is known about the effect of HIIT in children or adolescents, particularly its interaction with asthma. Not only is HIIT in adolescents with asthma insufficiently described, to the best of the author's knowledge, no pure HIIT intervention, with short aerobic intervals and equal rest periods, has been administered to adolescents with asthma. 
Enjoyment of an exercise intervention is key for attendance and adherence and therefore interventions should be co-developed with the participants. Furthermore, there are few inclusive interventions which combine participants both with and without asthma, which is a necessity to avoid stigma. Therefore the aim of the thesis was to co-develop and investigate the influence of an inclusive school-based HIIT intervention on the cardiorespiratory fitness of adolescents with asthma, with the intention of furthering our knowledge in exercise training as a means of increasing asthma control and quality of life. 


\section{CHAPTER 3}

\section{GENERAL METHODS}




\section{Chapter 3:General Methods}

This chapter describes the general methods of this thesis. For each individual study outlined in the thesis further specific detail is given within. All measures were performed at satellite laboratories at each of the respective schools.

\subsection{Participants}

The number of participants required to sufficiently power the statistical comparisons were estimated according to the method presented by Vincent (1999). To calculate the number of participants required to power the intervention study, the Paediatric Asthma Quality of Life Questionnaire was used as the primary outcome variable. It was calculated that, to achieve $80 \%$ power with the effect size of 0.5 (confidence level 0.05), a sample of 132 participants would be required. Due to the prevalence of asthma and the pragmatic nature of only being able to conduct the intervention in one school, 44 participants with asthma were required for the intervention with the remaining 88 required as controls. In order to account for drop out, a target number of 158 was set to allow for up to a $20 \%$ attrition rate (Lubans et al., 2016). To increase the statistical power of the study, two healthy participants were sought to be recruited for every one adolescent with asthma to both the intervention and the control schools. For the more sensitive sub-sample measures, to achieve an $80 \%$ power and 0.05 confidence level, eight participants were required in each group.

Fifteen schools were selected from Swansea and the surrounding area, based on size, location and socioeconomic status (free school meals). These schools were recruited via email, telephone and face-to-face meetings. One school was randomly selected to be the intervention, with stratified randomisation used to select four schools to act as a control to best match the free school meal status of the intervention school. Free school meal status was based on the most recent Estyn reports. Due to academic commitments, the intervention school was unwilling to allow the year 11 pupils to participate in the study, therefore, only pupils from years 7 to 10 were invited to participate. Initially, in line with the power calculations, 616 adolescents volunteered to participate in the study, 221 to receive the intervention (47 with asthma) and 395 
(106 with asthma) to act as the control. Possibly due to the name of the project (the eXercise for Asthma study with Commando Joe's), there was a potential selection bias, and therefore more adolescents with asthma agreed to participate than was expected given that approximately 1 in 11 children in the UK suffer with the condition (21\% and $27 \%$ with asthma in the intervention and control groups, respectively).

For sub-sample measures, stratified randomisation was used to select 10 participants with and 10 without asthma from each school year group of the intervention school; these were age and sex matched with participants from one of the control schools. It was decided to exceed the amount needed to power the statistics to allow for drop out and to provide greater confidence in the potential findings, however, due to time constraints, this was limited to 15 in each group (asthma and non-asthma, intervention and control). A flow chart outlining the school recruitment process, group allocation and intervention completion rate is included within Figure 3.1.

\subsection{Ethical approval}

Ethical approval for all experimental chapters was granted in agreement with the guidelines and policies set out by the Swansea University research ethics committees in both the College of Engineering and the Medical School (ref: PG/2014/29 and 140515). Information sheets (Appendix A) were developed for the Head teacher, parents/guardians and the participants, with appropriate language used for each target audience. Head teacher consent was provided from every school, with parent/guardian consent and participant assent collected prior to the commencement of the study. Further parental/guardian consent and participant assent was collected from the sub-sample for further physiological measures and each participant of the sub-sample was asked to assent at every time point. 


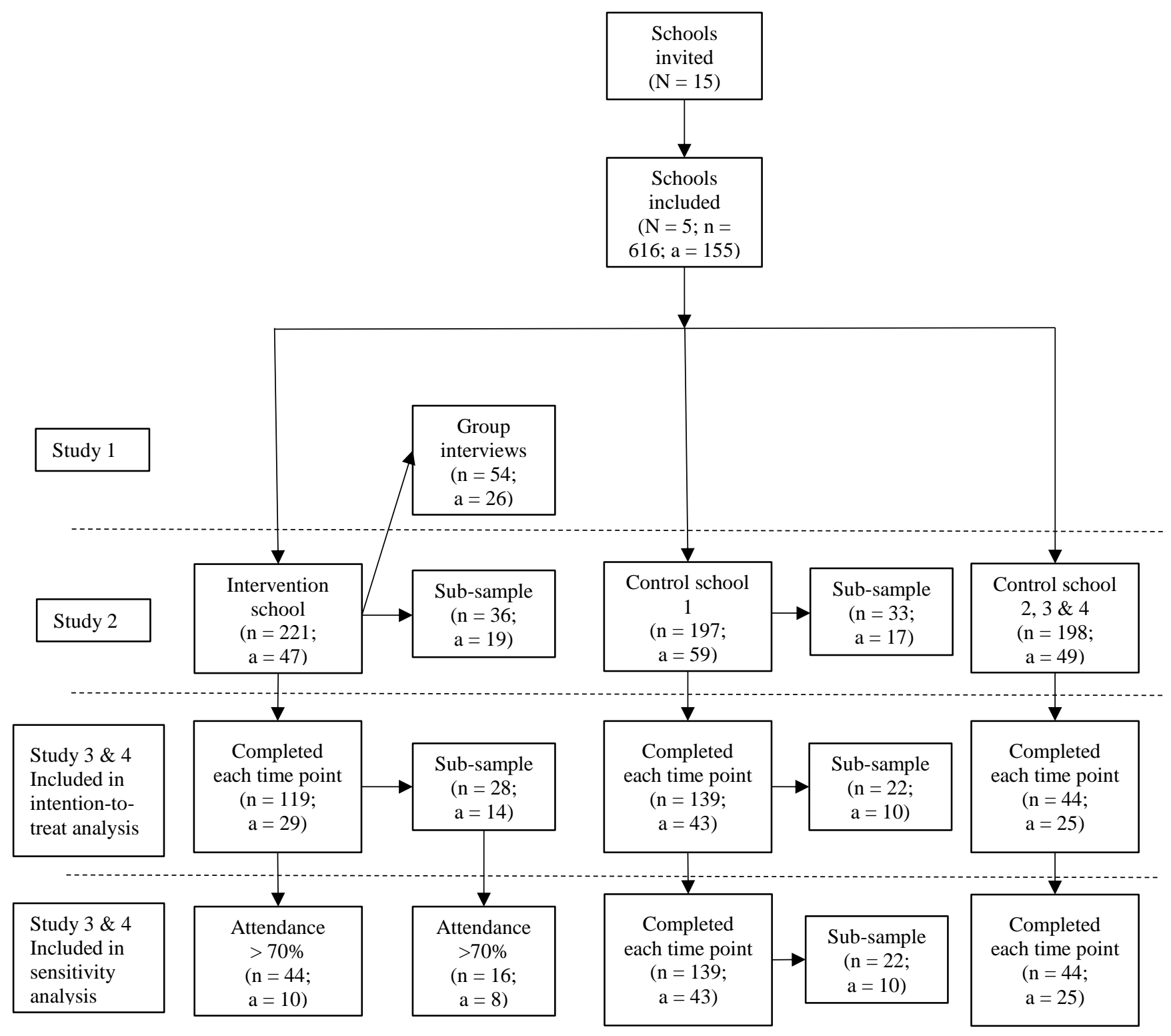

Figure 3.1. Flow chart outlining the school recruitment process, group allocation and intervention completion rate; $N=$ schools; $\mathbf{n}=$ total participants; $\mathbf{a}=$ participants with asthma (from $\mathbf{n}$ ) 


\subsection{Asthma and Severity}

Within the information sheets and the consent and assent forms a box was provided to indicate if the participant had asthma as diagnosed by a doctor. This box appeared on both the parental consent and the participant assent.

Each participant that indicated that they had asthma was provided with a healthcare utilisation questionnaire (Appendix C) to establish what medication they were prescribed and how often it was administered at each time point throughout the intervention. Asthma severity was assessed using the Global Initiative for Asthma guidelines (Global Initiative for Asthma, 2017) and classified as mild, moderate or severe according to the medication step required to achieve asthma control.

\subsubsection{Asthma medication}

All of the 155 adolescents with asthma took short-acting $\beta_{2}$ agonists (SABA) to control their asthma, in $20 \%$ this was their only medication for their condition, indicating mild asthma, assuming appropriate treatment. Seventy percent of the participants with asthma were on a combination of SABA and inhaled corticosteroids (ICS), with $10 \%$ indicated to have moderate-to-severe asthma on SABA, ICS and long-acting $\beta_{2}$ agonists (LABA). Moderate and severe asthma was grouped together in order to provide greater power to make any meaningful comparisons between severity groups.

Bronchodilators or other asthma medication were not withheld at any point during the measures as it was perceived as a safety risk. If not correctly medicated, the exercise measures may have acted as a trigger for asthma attacks. This may have had an impact on the results and should be taken into consideration when interpreting the results. 


\subsection{Instruments and procedures}

With the exception of the group interviews conducted in study 1, the measures were conducted over four time points, baseline, mid-intervention (3 months), postintervention (6 months) and at follow up (9 months).

\subsection{Wider-sample measures}

\subsubsection{Age}

Decimal age was calculated between the date of birth of the participant and the first day of measures at each time point, to the nearest 0.1 years.

\subsubsection{Anthropometry}

All anthropometrical measures were taken according to the techniques outlined by the International Society for the Advancement of Kinathropometry (Stewart et al., 2011). Participants were required to be in minimal clothing and without shoes. Body mass was measured to the nearest $0.1 \mathrm{~kg}$ using electronic weighing scales (Seca 876, Hamburg, Germany). Stature and sitting stature were measured to the nearest $0.01 \mathrm{~m}$ using a stadiometer (Seca 213, Hamburg, Germany). Participants were instructed to position themselves with their back against the stadiometer, heels together (for stature), looking straight ahead and the measurement was taken at maximal inhalation. Lower limb length was calculated as the difference between stature and sitting stature. Arm span was measured by placing the fingertip of one hand in the corner against the wall and to stretch with level arms as far as they could, this was measured to the nearest $0.01 \mathrm{~m}$. Waist circumference was measured using a tape measure to the nearest $0.01 \mathrm{~m}$ (Seca 203, Hamburg, Germany). The measure was taken at the waist, at the most indented point of the torso between the bottom of the rib cage and the iliac crest. 


\subsubsection{Maturation}

Pubertal maturity was self-assessed in accordance with the secondary sex characteristics outlined by Tanner (1962), which depict the 5 major stages of physical development from pre-puberty to post-puberty (Appendix X). This measure was completed in private and returned to the researchers in an unidentifiable envelope. This method of self-assessment has been shown to be valid and reproducible (Chan et al., 2008; Morris \& Udry, 1980; Taylor et al., 2001; Norris \& Richter, 2005; Schmitz et al., 2004) with the added advantage of this method over a physician assessment being that a trained nurse is not required. In addition to the Tanner stages self-assessment, the age at peak height velocity was calculated to provide an additional indicator of maturation status, according to the equations for both boys and girls developed by Mirwald et al. (2002; Equation 3.1 and 3.2). The $95 \%$ confidence intervals reported by Mirwald et al. (2002) were \pm 1 year and therefore in the current thesis where multiple measures are taken within a year, the results may not fully represent the maturity changes of the participants over time. The use of two measures of maturity status were utilised as verification indicators of each other, in addition to be used as covariates in additional analysis to avoid collinearity, for example, analysing body mass index.

Equation 3.1

For boys

Maturity Offset

$$
\begin{aligned}
& =-9.236+(0.0002708 \times(L L \times S S)) \\
& +(-0.001663 \times(\text { age } \times L L))+(0.007216 \times(\text { age } \times S S) \\
& +(0.02292 \times((B M \div S) \times 100))
\end{aligned}
$$


Equation 3.2

For Girls

Maturity Offset

$$
\begin{aligned}
& =-9.376+(0.0001882 \times(L L \times S S)) \\
& +(0.0022 \times(\text { age } \times L L))+(0.005841 \times(\text { age } \times S S) \\
& +(-0.002658 \times(\text { age } \times B M))+(0.07693 \\
& \times((B M \div S) \times 100))
\end{aligned}
$$

LL = Leg Length; $S S=$ Sitting Stature $; B M=$ Body Mass $; S=$ Stature

\subsubsection{Body Mass Index}

Body Mass Index (BMI) was calculated for each individual as an estimate of adiposity using the following equation.

Equation 3.3.

$$
\text { Body Mass Index }=\text { Body Mass }(\mathrm{kg}) \div \text { Stature }\left(\mathrm{m}^{2}\right)
$$

As all the participants were under the age of 18 years, BMI scores were grouped according to Barlow et al. (2007) using age and sex specific child percentiles based on growth charts. Overweight and obesity were defined as a BMI $>85^{\text {th }}$ and $>95^{\text {th }}$ percentile, respectively. As BMI is the most commonly used biomarker for obesity, it allows this research to be compared to the wider literature, it is easy to perform in large numbers and is inexpensive. Whilst skinfold thickness and dual-energy X-ray Absorptiometry (DXA) were considered for the research project, they were found to be too time consuming and specifically for DXA, as the participants were not clinical, it was not considered ethically sound. Whilst Guibas et al. (2013) suggest waist circumference should be used in child asthma populations between the ages of 2 and 5 years, BMI was found to be more appropriate in older children and 
adolescents due to the "adiposity rebound" observed post pre-school. For the current thesis, waist circumference was also measured to add to the findings of BMI as suggested by Lu et al. (2016).

\subsubsection{Physical activity}

Physical activity levels were measured objectively using ActiGraph GT3X+ accelerometers (ActiGraph, Pensacola, FL, USA) to record the quantity, intensity and frequency of body movement. Due to the size of the study, the GT3X+ was selected, possibly the leading instrument for measuring energy expenditure (Butte et al., 2010), as it has been found to be both a valid and reliable measure of physical activity (Trost, 2007) and is considerably cheaper than other measures. The accelerometers recorded at $100 \mathrm{~Hz}$ and were worn on each participant's right hip for seven consecutive days. Participants were instructed to remove the monitor if they undertook water-based or contact activities. When the monitor was removed, wear time diaries were used to log for how long and why. The data were analysed using Kinesoft version 3.3.67 (Kinesoft, Saskatchewan, Canada) employing 1 second epochs with sustained periods of at least 20-minutes of consecutive zeros considered non-wear time (Catellier et al., 2005). A minimum daily wear time of 10 hours per day for 2 weekdays and 1 weekend day was used (Rich et al., 2013). Physical activity intensities were calculated using Evenson et al. (2008) cut points, which have been shown to be valid and reliable determinates of activity intensity in children and adolescents (Trost et al., 2011). Whilst non-wear time is a significant problem, the use of objective measures of physical activity were chosen over selfreport due to the accuracy of measure whilst worn in comparison to self-reports of physical activity which require an understanding of what constitutes the differing intensities.

\subsubsection{Lung function}

Forced Expiratory Volume in 1 second $\left(\mathrm{FEV}_{1}\right)$, Forced Vital Capacity (FVC), $\mathrm{FEV}_{1} / \mathrm{FVC}$ ratio, Peak Expiratory Flow (PEF), and Forced Expiratory Flow between $25-75 \%$ of vital capacity $\left(\mathrm{FEF}_{25-75}\right)$ was measured using a portable dry spirometer 
(Alpha, Vitalograph, Buckingham, UK) with participants seated. Prior to testing on each day, the device was calibrated using a 31 syringe (Vitalograph, Buckingham, UK). All spirometric pulmonary function assessments were performed in line with recommendations according to American Thoracic Society guidelines (1995) and to the standardised protocol (Miller et al., 2005). The best of three measurements were taken and expressed as a percentage of the age-sex-stature predicted value (Rosenthal et al., 1993).

Participants were asked to sit up straight, breathe in as deeply as possible, place their lips around the mouthpiece tube and, when they were instructed, "blow out" into the mouthpiece as hard and as fast as possible until no further air could be exhaled; this was explained and demonstrated before the test. Each participant was asked to complete 3 "acceptable" tests, requiring each exhalation to be performed within $5 \%$ of each other, or until respiratory fatigue which was usually deemed to be at 6 or more breaths; in which case, they were asked to return the following day. Due to the "acceptable" tests being within $5 \%$ or $150 \mathrm{ml}$ for $\mathrm{FVC}$, whichever is greatest, the reproducibility at each time point should be taken into consideration when evaluating changes over time. Furthermore, the coefficient of variation for $\mathrm{FEV}_{1}, \mathrm{FVC}$ and $\mathrm{FEV}_{1} / \mathrm{FVC}$ were $25-26 \%, 24-25 \%$ and $8-10 \%$, respectively, which were lower than previously reported by Wicher et al., 2010 (33\%, 35\% and 11\%), but still has a large variation and should be considered when assessing the measure across time points. In addition, the standard error of the estimate for $\mathrm{FEV}_{1}, \mathrm{FVC}$ and $\mathrm{FEV}_{1} / \mathrm{FVC}$ were 0.03-0.05, 0.04-0.07 and 0.34-0.67, respectively, far less than reported by Wicher et al. (2010; 0.11, 0.61 and 6.34), although this could be due to the fewer participants within the study.

\subsubsection{Fractional exhaled Nitric Oxide}

Fractional exhaled nitric oxide (FeNO) was measured prior to spirometric testing in only those adolescents with asthma. The FeNO test was performed in accordance with the American Thoracic Society guidelines (Dweik et al., 2011). Participants were asked to completely exhale and then inhale to total lung capacity through the device (NIOX MINO, Aerocrine AB, Solna, Sweden), before immediately exhaling for 10 seconds at $50 \pm 5 \mathrm{ml} \cdot \mathrm{s}^{-1}$. Visual and audio cues were provided by the 
computer software throughout. One test was completed at all time-points except follow-up. The final three seconds of exhalation were evaluated.

Whilst tissue biopsy is the definitive measure of airway inflammation (Wadsworth et al. 2011), it is highly invasive, time consuming and requires high level of expertise. Therefore, using a less invasive means of obtaining airway biomarkers are more desirable which led to the use of FeNO in the current study. Whilst FeNO is an expensive measure, it is the most widely used exhaled biomarker of airway inflammation (Wadsworth et al. 2011), is quick to use and is highly reproducible in individuals (Holz et al. 2009), making it useful in the longitudinal intervention study. As FeNO is entirely dependent on the participant, it is associated with a very large $\mathrm{CV}$ of $103 \%$ with a SEM of $3.73 \mathrm{ppb}$, however, this is expected of the biomarker as evidence by previous research with a CV of $81 \%$ and a SEM of $9.87 \mathrm{ppb}$ (Moreira et al., 2008). Although, as there is such a large variability, this biomarker is less reliable for finding differences between time points.

\subsubsection{Cardiorespiratory fitness}

Cardiorespiratory fitness was estimated using the 20-metre progressive shuttle run test, a previously validated field measure in children (Mayorga-Vega et al., 2015). The measure involved continuous running between two lines 20 metres apart in time to recorded beeps increasing in speed throughout the test. The number of shuttles completed before voluntary exhaustion were recorded and compared as absolute number of shuttles.

Whilst the Yo-Yo intermittent recovery level 1 test was perhaps more aligned with the intermittent design of the exercise intervention, the logistics of the test were deemed to be an issue. Indeed, the measure required a length of greater than 30 metres which was not able to be achieved in each school's sports hall, limiting the project to the 20-metre shuttle run. However, the continuous nature of the 20 -metre shuttle run matched the design of the incremental ramp test for those in the subsample measure, perhaps increasing the comparability of the two tests.

The CV shown by the 20-metre shuttle run demonstrated large variability of the measure (50-54\%) with a SEM of 1.2-1.9 shuttles, however this is expected as 
shown in a previous study $(\mathrm{CV}=48-52 \%, \mathrm{SEM}=0.42$ shuttles; McNarry et al., 2014).

\subsubsection{Questionnaires}

Questionnaires were administered in a classroom setting with the same instructions given for each group at each time point. Participants were able to answer within their own time and give their own answers. If queries arose around the questionnaire, the participants were able to ask for help from the research team. Internal consistency of each questionnaire was measured using Cronbach's alpha coefficients (Cronbach, 1951) in each chapter.

\subsubsection{Asthma control}

Asthma control was assessed using the Asthma Control Questionnaire (ACQ, Juniper et al., 2010) which consists of 7-items focusing on reliever inhaler use, symptoms and FEV1 score. Items of the ACQ were scored from 0 to 6, with ACQ scores of $\leq 0.75$ or $\geq 1.5$ indicating well-controlled and poorly-controlled asthma, respectively. The ACQ has been validated in children between the ages of 6 and 16 (Juniper et al., 2010) and was found to be responsive to chance in asthma control with a minimal important difference of $0.52 \pm 0.45$.

Whilst there is no consensus for a gold standard of asthma control using a questionnaire, the addition of a physiological measure in the ACQ (FEV 1 ) and the use of the questionnaire in the BTS sign guidelines for the assessment of asthma control in adolescents, were the reasons why the questionnaire was selected for this project. In addition, the GINA asthma control questionnaire did not provide a minimal important difference and therefore would present issues when assessing any changes over time in comparison to the ACQ.

\subsubsection{Asthma quality of life}

Symptoms, activity limitation and emotional and environmental effects of asthma were assessed using the Paediatric Asthma Quality of Life Questionnaire (PAQLQ, Juniper et al., 1996). The PAQLQ consists of 23 questions (scored on a Likert scale from 1 to 7), with a higher score indicative of a better asthma status. The questions 
are divided into 3 domains of activity limitations, symptoms and emotional function. The minimal important difference is 0.5 .

As with the asthma control questionnaires, there are numerous quality of life questionnaires, however the two which were debated for this project were the PAQLQ and the PedsQL Asthma module. Whilst the PedsQL would possibly have been a better choice to compare to the quality of life questionnaire administered to the wider population, it lacked questions on participation in normal activities which were in the PAQLQ. Recall is also an important factor when administering questionnaires and the month period for the PedsQL asthma module was deemed too long in comparison to the PAQLQ. Finally the PAQLQ is the most widely used questionnaire (Wilson et al. 2012) so provides a better comparison to previous studies.

\subsubsection{Quality of life}

In addition to asthma related quality of life, a further quality of life measure was used to compare the participants with asthma to their healthy peers and measure any quality of life changes in healthy adolescents due to the intervention. The Pediatric Quality of Life Inventory (PedsQL) Teenager Report (Version 4.0, Varni et al., 1999) is a widely validated measure in adolescents (Varni et al., 2006, Varni et al., 2003, Varni et al., 2001). The 23-item PedsQL consists of questions on the participants' physical, emotional, social and school functioning quality, with higher scores indicating a better quality of life. There are other quality of life questionnaires, such as KIDSCREEN (Ravens-Sieberer et al., 2005) which is also a valid measure (Janssens et al., 2008), however, due to the length of the questionnaire and limited time of the project, the PedsQL was deemed the most pragmatic choice.

\subsection{Sub-sample measures}

For each of the sub-sample exercise tests, participants were required to perform each test at the same time of day ( \pm 2 hours). Participants were instructed to arrive in a rested and hydrated state and at least 2 hours postprandial. 


\subsubsection{Incremental ramp tests}

The incremental ramp tests were performed on an electromagnetically braked cycle ergometer (Ergoselect 200, Ergoline GmbH, Lindenstrasse, Germany). Whilst a treadmill may have matched the exercise performed in the intervention and possibly detected more subtle changes in the specific muscle groups, the cycle ergometer was selected for pragmatic reasons. Indeed, the cycle ergometer was selected as it is associated with a decreased movement artefact, increasing the quality of the signals obtained from the ECG and NIRS devices. Moreover, the cycle ergometer is able to make smaller changes in work rate and more closely match the target work rate associated with heavy-intensity for the step exercise tests. Furthermore, as the measures were taken in satellite labs in each school, we were unable to transport a treadmill to each school within the time constraints of getting all of the measures done.

The handle bar height, distance from the participant and seat height were adapted to suit each participant. The ramp rate was individually targeted to each participant ranging from $12-24 \mathrm{~W} \cdot \mathrm{min}^{-1}$ in order to produce a test duration of $8-12$ minutes (Buchfuhrer et al., 1983). The ramp rates were estimated from pre-baseline familiarisation incremental ramp tests. The incremental ramp test began with 3 minutes of "unloaded" pedalling $(0 \mathrm{~W})$ followed by the predetermined ramp rate. Throughout the test, the participants were told to keep the cadence between $70-80$ rpm with a visual cue and this was verbally reinforced by the researchers if not adhered to. Towards the end of the test strong verbal encouragement was given by the researchers and the test was terminated on volitional exhaustion, defined as a drop in cadence $>10 \mathrm{rpm}$ for five consecutive seconds. Secondary criteria for assessing peak $\dot{V} \mathrm{O}_{2}$ included, respiratory exchange ratio $>1.0$, peak heart rate $>85 \%$ age predicted heart rate maximum and a rate of perceived exertion $>17$ on the Borg scale (Armstrong \& Welsman 1994).

Adolescents with asthma have previously demonstrated a CV of 20\% and SEM of 0.071 for peak $\dot{V} \mathrm{O}_{2}$ (van Veldhoven et al., 2001), which show similar re-test reliability as the current study $(\mathrm{CV}=23.8 \%, \mathrm{SEM}=0.081)$. 


\subsubsection{Step Exercise Tests}

The step exercise tests were performed on the same equipment as the incremental ramp test and the handlebar height, distance and saddle height were adjusted to match the participants previously recorded measures for that specific time point. The study employed heavy-intensity work rate for the step tests for numerous reasons: i) It most closely matched the high-intensity nature of the intervention whilst still being tolerable in comparison to severe-intensity, which might not provide a sufficient duration for analysis; ii) there is a greater amount of literature to compare it to for both training and youth; iii) it requires fewer transitions than moderate-intensity due to the higher signal to noise ratio and iv) due to the intensity being above GET, the slow component is observable. The step exercise tests involved 6 minutes of unloaded pedalling $(0 \mathrm{~W})$, followed by an abrupt transition to a heavy-intensity work rate calculated as $40 \%$ of the difference between the GET and peak $\dot{V} \mathrm{O}_{2}(\Delta 40 \%)$ for 6 minutes. During the cycling, a cadence of 70-80rpm was required. Each participant completed 3 transitions, each separated by 24 hours at each time point. The $95 \%$ confidence intervals associated with the $\dot{V} \mathrm{O}_{2}$ time constant for the three transitions were within \pm 5 seconds, satisfying the criteria proposed by Fawkner at al. (2007).

The test-retest reliability of the $\dot{V} \mathrm{O}_{2}$ response to a step change in heavy-intensity exercise was assessed and revealed a CV of 16-22\% and SEM of 1.3-2.1s. These results were similar to, but perhaps slightly better than, data reported in previous $\dot{V} \mathrm{O}_{2}$ kinetics time constant CV of $28 \%$ and SEM of 2.8s (Marwood et al., 2010).

\subsubsection{Measurement of gas exchange parameters}

For both the incremental ramp and exercise tests, gas exchange variables were measured on a breath-by-breath basis (Jaeger Oxycon Mobile, Jaeger, Hoechberg, Germany) using a facemask with low dead-space connected via an impeller turbine assembly (Jaeger Triple V, Hoechberg, Germany). Gas analysers were calibrated prior to each test with gases of known concentrations $\left(15.0 \% \mathrm{O}_{2}\right.$ and $\left.5.0 \% \mathrm{CO}_{2}\right)$ and the turbine volume transducer was calibrated using a built-in function calibrated using a 31 syringe (Hans Rudolph, Kansas City, MO). The analyser also recorded ambient air temperature and pressure prior to each test. The volume and concentration signals were time-aligned by accounting for the delay in capillary gas 
transit and analyser rise time relative to the volume signal. The inspired and expired gas volumes and concentration signals were continuously sampled at $100 \mathrm{~Hz}$.

Breath-by-breath $\dot{V} \mathrm{O}_{2}$ measurements during peak $\dot{V} \mathrm{O}_{2}$ measures using the Oxycon mobile have been reported to be reproducible, with coefficients of variation ranging from 3.4-6.3\% (Carter \& Jeukendrup, 2001, Rosdahl et al., 2010).

\subsubsection{Measurement of cardiac parameters}

Heart rate was recorded continuously during both the incremental and step exercise tests at a sampling frequency of $250 \mathrm{~Hz}$ (Physio Flow PF-05 Lab1, Manatec Biomedical, France). Physio Flow was used instead of the standard Polar heart rate monitors as for the wider project, heart rate variability was measured which required a six-lead system.

\subsubsection{Measurement of muscle oxygenation parameters}

The oxygenation status of the right m.vastus lateralis was also monitored during each step exercise test to non-invasively assess the deoxygenated haemoglobin ([HHb]) dynamics using near-infrared spectroscopy (Portamon, Artinis Medical Systems, Netherlands). The Portamon device consisted of three light sources emitting two wavelengths (760 and $850 \mathrm{~nm}$ ) and a photon detector. The reflected light was recorded continuously at $10 \mathrm{~Hz}$ and used to estimate [HHb]. The changes in the deoxygenation signal indicate changes in local $\mathrm{O}_{2}$ extraction kinetics (Koga et al., 2012). The Portamon device was placed at the midpoint of the muscle using micropore tape (3M, Maplewood, $\mathrm{MN}$ ); to minimise movement and the interference of extraneous light, a bandage was wrapped around the Portamon device and leg. The Portamon device has been reported to produce acceptable reproducibility estimates (Shadgan et al., 2009).

\subsubsection{Determination of the gas exchange threshold and $\Delta 40 \%$ work rate}

The GET was determined from the incremental ramp test using the V-slope method (Beaver et al., 1986) which assesses the point at which carbon dioxide production begins to increase disproportionately to $\dot{V} \mathrm{O}_{2}$. To identify the GET a purpose designed software developed using LabVIEW (National Instruments, Newbury, UK) 
was utilised. The first 60 seconds of the data was excluded following the commencement of the ramp function and any data past the respiratory compensation point was removed. A plot of $\dot{V} \mathrm{CO}_{2}$ against $\dot{V} \mathrm{O}_{2}$ was produced and the GET was identified as the intersection point between the two regression lines, which was then displayed graphically and visually confirmed by the researchers.

Subsequently, the work rate for heavy-intensity domain was calculated from the GET. The work rate that would elicit $40 \%$ of the difference between GET and peak $\dot{V} \mathrm{O}_{2}(\Delta 40 \%)$ was determined, accounting for the mean response time for $\dot{V} \mathrm{O}_{2}$ during ramp exercise (i.e., two thirds of the ramp rate was deducted from the work rate at the GET and peak (Whipp et al., 1981)).

\subsubsection{Analysis of $\dot{\mathrm{V}} \mathrm{O}_{2}$ response to step exercise tests}

Breath-by-breath $\dot{V} \mathrm{O}_{2}$ responses were first examined using a 5-second moving average to identify and remove any errant breaths caused by coughing, swallowing, sighing, for example, which were more than four standard deviations from the local mean. Each transition was then interpolated to 1-second intervals, time-aligned to the start of exercise and averaged to produce a single response profile for each timepoint to improve the signal to noise ratio.

To remove the influence of the cardiodynamic phase (phase I) on the analysis of the $\dot{V} \mathrm{O}_{2}$ response, the first 15 seconds of the response to the heavy-intensity exercise were excluded. Only 15 seconds were removed as in children and adolescence the response to exercise is shorter in comparison to adults ( 20 seconds) on the basis of findings of Hebestreit et al. (1998), Springer et al. (1991) and Fawkner et al. (2002).

Each step exercise profile was then corrected for baseline $\dot{V} \mathrm{O}_{2}$ and a monoexponential model applied (Equation 3.4):

Equation 3.4.

$$
Y_{(t)}=A_{1}\left(1-e^{-\left(t-\delta_{1}\right) / \tau_{1}}\right)
$$


Equation 3.5.

$$
Y_{(t)}=A_{1}\left(1-e^{-(t) / \tau_{1}}\right)
$$

where $\mathrm{Y}$ is the increase in the parameter $\left(\dot{V} \mathrm{O}_{2},[\mathrm{HHb}]\right.$ and $\left.\mathrm{HR}\right)$ at time $t$ above the baseline value (calculated as the mean of the first 45-seconds of the last minute of baseline pedalling). A1, $\delta 1$ and $\tau 1$ are the primary component amplitude, time delay (which was allowed to vary freely), and time constant, respectively. Variables derived from the mono-exponential (A1, $\delta 1$ and $\tau 1$ ) and their $95 \%$ confidence intervals were determined by least squares non-linear regression analysis (Graphpad Prism, Graphpad Software, San Diego, CA). A mono-exponential model was selected as a bi-exponential model was found to produce an ambiguous fit to the data. Purpose-designed custom software was then used to iteratively fit a singleexponential function to the $\dot{V} \mathrm{O}_{2}$ data until the window encompassed the entire exercise response. The resulting phase II time constants were plotted against time to identify the point at which the phase II time constant consistently deviated from the previously "flat" profile providing the start time of the slow component (Fawkner \& Armstrong, 2004b). The amplitude of the $\dot{V} \mathrm{O}_{2}$ slow component was determined as the difference between the $\dot{V} \mathrm{O}_{2}$ at end of phase II and at end exercise $(\mathrm{t}=360)$ and presented in absolute terms and as a percentage of end exercise $\dot{V} \mathrm{O}_{2}$. Finally, the mean response time (MRT) was calculated by fitting equation 3.5 , from $t=0$ to $\mathrm{t}=360$.

\subsubsection{Analysis of the heart rate and [HHb] response}

The $[\mathrm{HHb}]$ and HR responses to the step exercise tests were modelled using a monoexponential function. Each transition was interpolated to 1-second intervals, timealigned to the start of exercise and averaged to produce a single response profile for each measurement time-point. The $[\mathrm{HHb}]$ data were baseline averaged, expressed as a percentage of end exercise amplitude and then averaged into 5-second time bins. The [HHb] was modelled using Equation 3.4 with the time delay identified as the time after exercise onset at which $[\mathrm{HHb}]$ began a systematic increase above the nadir value (Murias et al., 2016). The mono-exponential function was fitted between the identified time delay and time at which end of phase II was identified by the mono- 
exponential model of the $\dot{V} \mathrm{O}_{2}$ kinetics. The HR response was modelled by both Equations 3.4 and 3.5, as per previous research (McNarry et al., 2017, Murias et al., 2010); however, Equation 3.4 was selected for analysis as it was deemed the superior fit for $91 \%$ of transitions (Graphpad Prism, Graphpad Software, San Diego, CA). The model was fitted between $\mathrm{t}=0$ and $\mathrm{t}=360$. The $[\mathrm{HHb}$ ] time delay and time constant were subsequently summed to give the MRT. Additionally, to assess the fractional $\mathrm{O}_{2}$ extraction and $\mathrm{O}_{2}$ delivery, the $[\mathrm{HHb}] / \dot{V} \mathrm{O}_{2}$ ratio was calculated (Murias et al., 2011). Both [HHb] and $\dot{V} \mathrm{O}_{2}$ data were normalised for each participant, with the $\dot{V} \mathrm{O}_{2}$ left-shifted by 15 s to account for the cardiodynamic phase (Murias et al., 2010). Values found to be greater than 1.0 reflect a greater reliance on fractional $\mathrm{O}_{2}$ extraction and a poorer local $\mathrm{O}_{2}$ delivery relative to muscle $\mathrm{O} 2$ utilisation compared with the exercise steady-state (values $=1.0$ ). Each of the data sets were then averaged into $5 \mathrm{~s}$ time bins and the ratio of $[\mathrm{HHb}]: \dot{V} \mathrm{O}_{2}$ calculated for each time bin. Subsequently, the average of all the individual time bins was calculated to produce an overall "mean" ratio.

\subsubsection{Allometric scaling}

To account for body size, analysis of covariance (ANCOVA) was used to determine the allometric relationship between peak $\dot{V} \mathrm{O}_{2}$ and body mass using log transformed data. Common allometric exponents were confirmed for the data and power function ratios $(\mathrm{Y} / \mathrm{Xb})$ were computed. 


\section{CHAPTER 4}

\section{PERCEPTIONS OF ASTHMA AND EXERCISE IN ADOLESCENTS WITH AND WITHOUT ASTHMA}




\section{Chapter 4: Perceptions of asthma and exercise in adolescents with and without}

asthma

This Study has been disseminated as follows:

Publication: Winn CON, Mackintosh KA, Eddolls WTB, Stratton G, Wilson AM, Rance JY, Doull IJM, McNarry MA, Davies GA. Perceptions of asthma and exercise in adolescents with and without asthma. Journal of Asthma. 2017:30,1-9.

Oral presentation: Winn CON, Mackintosh KA, Eddolls WTB, Stratton G, Wilson AM, McNarry MA, Davies GA (2016). Perceptions of asthma and exercise in children and adolescents. Asthma UK Centre for Applied Research Annual Scientific Meeting; Edinburgh, UK

\subsection{Introduction}

Asthma is one of the most common chronic diseases in children in the UK (Wanrooij et al., 2014). The benefits associated with exercise are well-recognised for healthy children, with additional benefits, such as reduced symptoms and severity (Andrade et al., 2014), for those with asthma. However, whilst some children with asthma recognise that regular exercise is associated with improved control of their asthma and enhanced physical self-perceptions (Chiang et al., 2006), exercise participation has been cited in some studies as lower in those with asthma compared to their healthy peers (Glazebrook et al., 2006, Lang et al., 2004). Indeed, the attribution of normal symptoms of physical exertion to symptoms of asthma is a common misconception (Rietveld et al., 2010, Williams et al., 2010), which, when exacerbated by a lack of fitness (McNarry et al., 2014a), leads to a greater manifestation of the apparent symptoms of asthma, resulting in further avoidance of exercise and a vicious negative cycle.

Commonly cited barriers to exercise for adolescents include time constraints, school work and lack of interest (Tappe et al., 1989); with additional barriers, such as fear of asthma symptoms, in those with asthma (Rudell et al., 2012). Despite conflicting 
literature on fitness levels (Philpott et al., 2010; McNarry et al., 2014a; Berntsen et al., 2009), there is a perception that children with asthma are less physically able than their healthy peers (Sawyer \& Fardy, 2003), potentially reflecting a lack of understanding rather than true disease-related physiological limitation. Teachers report that they have limited confidence when engaging children with asthma in exercise and encourage them towards musical instruments rather than physical pursuits (Thornton, 1997). This perception is frequently reinforced by parents who restrict their children's activities to minimise potential detrimental risks of asthma (Callery et al., 2003, Dantas et al., 2014). These actions are likely to contribute to the feelings of ostracization often cited by those with asthma, with some children deliberately "struggling through" exercises with their healthy peers to avoid social isolation (Protudjer et al., 2012) and being identified as different (Trollvik et al., 2011).

Although numerous studies have described the perceptions of those with asthma regarding their ability to exercise and their perceived barriers and facilitators (Jonsson et al., 2014, Walker \& Reznik, 2014), there is little information on the perceptions of their healthy peers, which may impact participation. Furthering our understanding of the perceptions of those with and without asthma is fundamental to the design of interventions to increase exercise in adolescents with asthma, thus breaking the vicious negative cycle of exercise avoidance (Ram et al., 2005). Frequently reported barriers to exercise interventions revolve around poor adherence due to monotony and sustainability, for continuous aerobic exercise (Coquart et al., 2008), and high-intensity exercise interventions, respectively (Logan et al., 2014). It is noteworthy that no studies to date have sought input from adolescents with asthma themselves with regards to the intervention design and implementation.

The primary aim of this study was therefore to elicit views of adolescents, with and without asthma, about exercise and asthma, and the perceived benefits of and barriers to participation. The secondary aim was to inform the design of and codevelop a high-intensity interval training intervention described in Chapter 6 and 7. 


\subsection{Methods}

\subsubsection{Participants}

Fifteen secondary schools in the UK were invited, as part of the wider randomised control trial (the X4A trial: eXercise for Asthma with Commando Joe's), to participate in an exercise intervention with the aim of improving asthma symptoms and quality of life. Subsequently, one school was randomised to the intervention arm and four schools to the control arm of the study. The current Chapter describes a qualitative study that was conducted using semi-structured group interviews of adolescents with and without asthma. Ethical approval was granted by the institutional research ethics committee (ref: PG/2014/29).

Five hundred and eighty five adolescents from the intervention school were eligible to participate (aged 11-14 years) in the exercise intervention, of which 223 (48 with asthma; 24 boys) provided written parental consent and child assent (see appendix A). Using stratified randomisation, a subsample of 60 adolescents, split by age, sex and asthma, were selected to participate in formative group interviews. Three school year groups were used (11-12 years, 12-13 years and 13-14 years) with ten adolescents with and ten without asthma from each, with an even split of sex ( $\mathrm{n}=$ 60 ). From the 60 selected participants, six were absent on the day, and therefore 26 and 28 participants with (4 overweight, 4 obese) and without asthma (3 overweight, 2 obese), respectively, attended the interviews $(13.1 \pm 0.9$ years $)$. Of the 26 with asthma, severity was classified as intermittent and mild persistent (88\%) and moderate and severe (12\%) according to GINA criteria (Global Initiative for Asthma, 2017).

\subsubsection{Study design}

For the first experimental chapter, a qualitative approach was chosen to acquire an understanding of both those with and without asthma's perceptions on asthma and exercise and to inform the design of a HIIT intervention. Data have been collected by means of group interview and the study design is based on grounded theory (Glaser \& Strauss, 1967). Grounded theory is ideal for identifying categories, 
creating links and establishing relationships between them and provides an explanatory framework with which to understand the outcome (Crooks, 2001). This research process allows an interpretation of the raw data of group interview transcripts to emerge as themes via coding the transcripts. The questions were designed to elicit perceptions and ideas on high-intensity interval training and how best to implement this aimed at adolescents with asthma whilst still appealing to their peers. High-intensity interval training had previously been chosen to be implemented and the interviews were used to further develop the design of the intervention.

\subsubsection{Procedures}

Thirteen semi-structured group interviews consisting of 3-5 adolescents were conducted separately grouped by age and condition. The group interviews were performed in a quiet area of the school to avoid interruption; the interviews lasted $30.9 \pm 3.2$ minutes. Interviewers were all conducted by the same researcher (William Eddolls) for consistency, who was known by the participants, providing an interview environment in which the adolescents could speak honestly and freely about their perceptions (Hennessy \& Heary, 2005). Group interviews with adolescents have been found to be a viable method for exploring perspectives provided the groups are small (i.e., 3-5 participants, Gibson, 2007, Horner, 2000). The small group sizes alleviate issues associated with group interviews with adolescents such as controlling or managing the groups. In addition, when using group interviews certain participants may be hesitant to express their ideas, however, these groups were stratified by academic year group and split for asthma and non-asthma so that younger participants/participants with asthma did not feel intimidated within their group. Questions were structured around adolescents' knowledge, attitudes and beliefs towards asthma and its impact on exercise participation and life. The questions were designed to elicit the adolescents' perceptions of asthma and exercise, with questions in the asthma groups relating to themselves and others with asthma, and questions in the groups without asthma relating to their perceptions of those with asthma. Each group were also asked questions relating to the design of the high-intensity interval training intervention in which they were to participate. Participants were not limited to one answer per question and therefore the figures are 
not limited to 51 responses to the same theme. The number of participants recruited was based on previous qualitative work (Protudjer et al. 2012). Data saturation was deemed when analysing the interviews, no new themes were observed, whilst mounting instances of the same themes were given (Urquhart, 2013). Sample interview questions are presented in Table 4.1. All interviews were recorded using a digital recorder (Galaxy S7 Edge, Samsung) and were transcribed verbatim.

\subsubsection{Data analysis}

The transcribed data were thematically analysed in a deductive manner and presented via diagrams of key emergent themes (pen profiles), which are considered appropriate for representing findings from large data sets in a manner understandable to both qualitative and quantitative researchers (Mackintosh et al., 2011, Ridgers et al., 2012). The pen profiles were independently constructed by both myself (Charles Winn) and co-author (Dr. Kelly Mackintosh) prior to discussion on key themes, issues and findings (O'Dea, 2003). Following initial analyses, both authors presented the pen profiles to co-author (Dr. Melitta McNarry) for co-operative triangulation, whereby the data was cross-examined in reverse from pen profile to transcripts until a general consensus was reached. This process was repeated, allowing all authors to offer alternative interpretations of the data, until a final acceptable consensus had been reached. 
Table 4.1. Example interview questions

\section{Group interview Example questions}

What is asthma?
What do you think people without asthma get wrong or don't
understand?
How does asthma affect your general life?
How does your asthma affect you when participating in
How do you manage your asthma during exercise?
What activities do you find particularly difficult with asthma?
Do you have any particular worries about exercise and asthma
and why?
What is asthma?
How do you think asthma might affect a person's general life?
How do you think exercise affects people with asthma?
How do those with asthma manage it?
Do you think those with asthma have any particular worries?
Do you think people with asthma take part in as much physical
activity as you, and why?

HIIT Intervention - What do you most enjoy doing?

Both with and without asthma

What physical activity do you do?

What is your least favourite activity to participate in?

What do you think high-intensity interval training is?

What type of activities do you think would get your heart rate up high but be enjoyable?

How difficult do you think high-intensity interval training will be and why?

Where do you like to participate in exercise and what time of the school day would you like to participate?

What could stop you from being able to participate in the exercise sessions? 


\subsection{Results}

Participants quotes are labelled in text by sex $(\mathrm{B}=$ boy, $\mathrm{G}=$ girl $)$.

\subsubsection{Participants with asthma}

Key emergent themes have been structured around control, impact, perceptions and exercise (Figure 4.1). The group interviews revealed that the majority of adolescents with asthma controlled their condition using an inhaler (78\%). Other participants, and indeed those who could not access their inhaler during asthmatic symptoms, suggested altering their breathing pattern as a mechanism to control their asthma. Only one person highlighted taking their inhaler prior to an activity as a control strategy.

Asthma was found to impact adolescents during school and/or in a social context. For example, participants felt that their asthma restricted them whilst they were with their friends,

"I just want to keep up but then I can't" B4,

or resulted in them being left out altogether. Furthermore, some adolescents even reported struggling when laughing with their friends,

"I can't laugh a lot, it's really hard" G1.

Symptoms of asthma experienced at night resulted in poor sleep quality and consequently impacted on social aspects and a lack of concentration in school.

Poor school attendance due to asthma symptoms and doctors' appointments was reported, with particular focus on reducing their involvement, or participation, in physical education (PE) lessons. The majority of the participants alluded to their healthy peers having a lack of understanding of their condition and often misinterpreting situations. Specifically, those with asthma felt that they were often perceived as lazy or that they used their condition as an excuse,

"they think it might just be like an excuse" B3. 


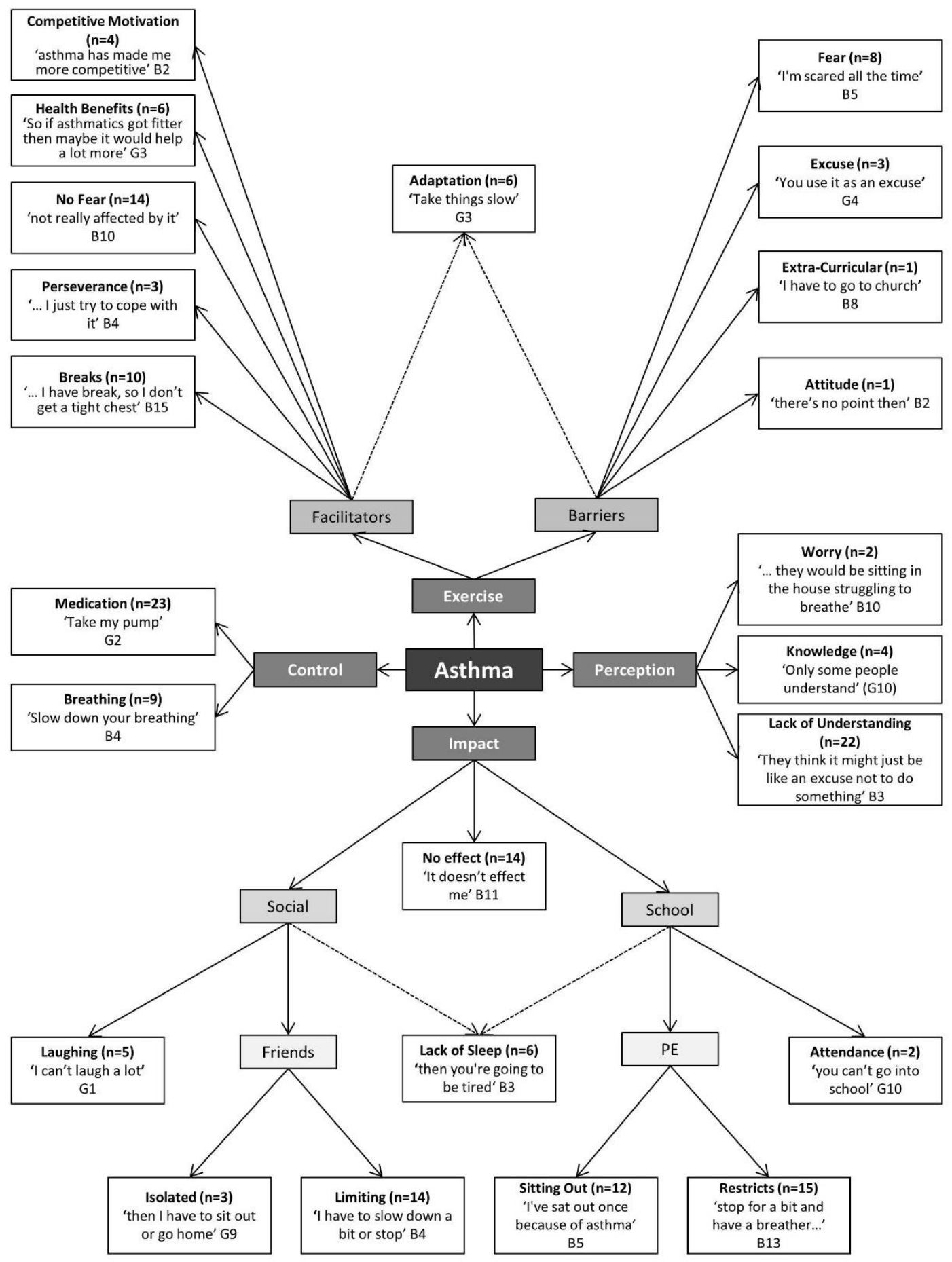

Figure 4.1. Adolescents with asthma - pen profile of perceptions of asthma and exercise

$\mathrm{B}=$ Boy $; \mathrm{G}=$ Girl PE $=$ Physical education 
Some participants reported being told to:

"get on with it" B3

by their healthy peers. Finally, some reported that healthy peers failed to appreciate when it was their asthma symptoms limiting them,

"they just misjudge everything, they think that you can do it when you can't" $B 2$.

Whilst some adolescents with asthma did acknowledge that they sometimes used their asthma as an excuse,

"When I don't want to do something I use it as an excuse" G4,

a more common perception was one of fear of exercise inducing an asthma attack leading to decreased participation,

"I do prevent myself from doing activity because I feel that I'm scared to have an asthma attack" G3.

In contrast, some reported that having asthma actually acted as a facilitator to exercise by increasing their competitiveness to show that they are not affected by their condition,

"I just want to do as much as everyone else" G2.

Moreover, six of the participants found that being 'fitter' reduced their asthma symptoms and therefore used exercise as a way to improve their health.

\subsubsection{Participants without asthma}

Similar to participants with asthma (Figure 4.1), key emergent themes have been structured around control, impact, perceptions and exercise (Figure 4.2). Those without asthma were less sure about how asthma is controlled; 39\% demonstrated knowledge of the use of inhalers and $11 \%$ suggested altering breathing as a form of asthma control. Similarly, healthy counterparts were also less clear on the impact of asthma on school and social life, only recognising the burden of carrying an inhaler 
and factors such as worrying about having an asthma attack. Eight adolescents without asthma (29\%) believed that asthma had no effect on social or school life,

“they've got their pump, it shouldn't really affect anything” G12.

Despite some thinking there was no effect, others noticed the issues during PE,

"they slow down, take their pumps and then get going again” B23.

School attendance was also noted as a potential issue for upcoming exams,

"they have to leave the class ... they can't afford to miss out on work" B16.

Two of the group had a complete lack of understanding of the condition, with five more not providing an answer to 'what is asthma?' Adolescents who had some understanding outlined breathing difficulties (68\%) and reduced lung function (14\%) as characteristics of asthma. Indeed, breathing difficulties were outlined as one of the main barriers to exercise for adolescents with asthma,

“they can't breathe properly" G16.

along with stopping often to take breaks (46\%). These breaks were perceived by others as laziness, using asthma as an excuse to avoid certain activities, especially in girls,

"many girls use it as an excuse because they don't want to have PE" G12

and as an excuse to avoid trouble, such as forgetting their kit. Other cited potential barriers included fear of asthma attacks (14\%) and not being able to participate in as much exercise $(21 \%)$. Participants without asthma perceived there to be relatively few facilitators of exercise in comparison to their peers with asthma, citing only health benefits $(18 \%)$. 


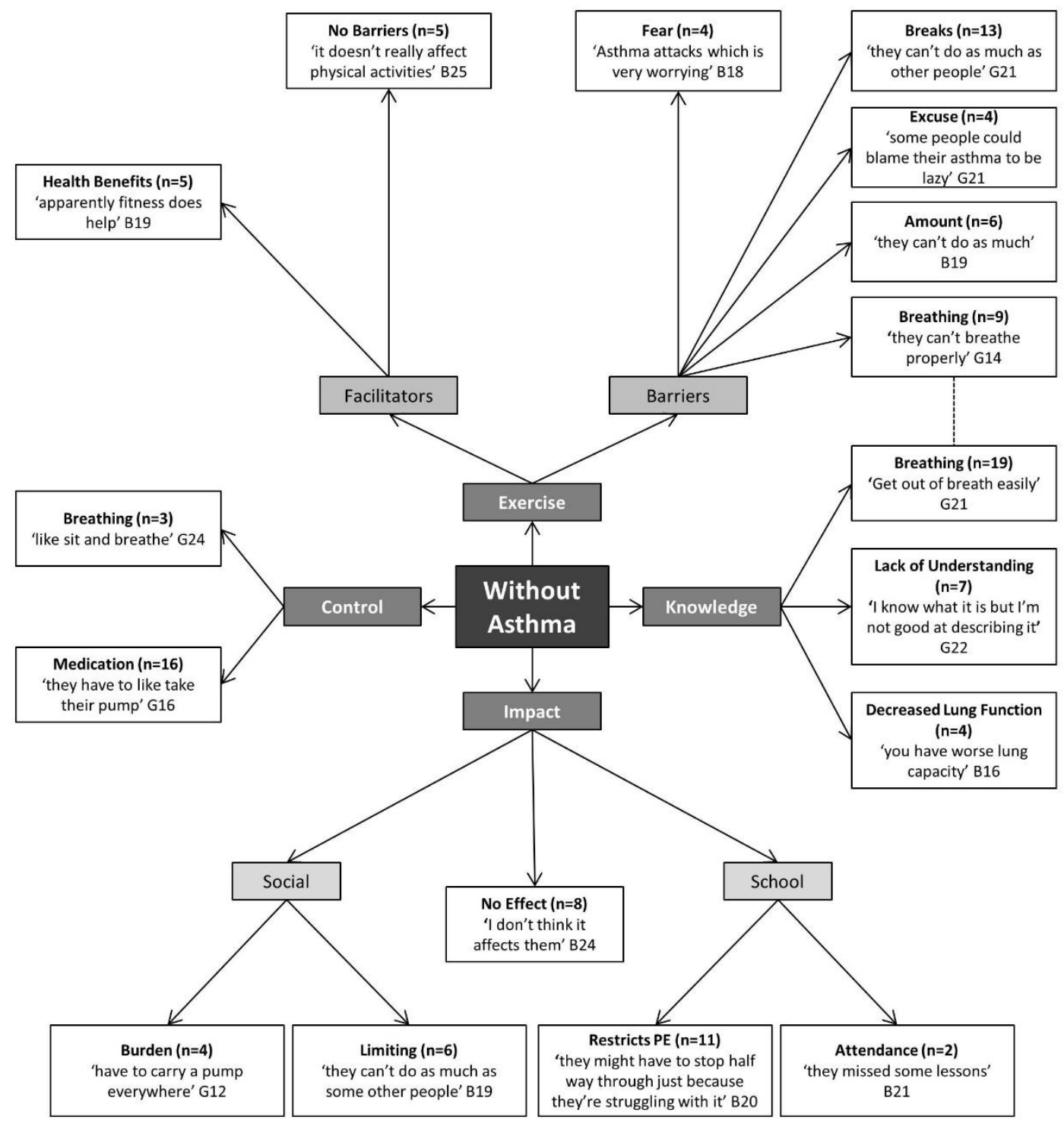

Figure 4.2. Adolescents without asthma - pen profile of perceptions of asthma and exercise

$\mathrm{B}=$ Boy $; \mathrm{G}=$ Girl $; \mathrm{PE}=$ Physical education 


\subsubsection{Views on an exercise intervention}

In response to the icebreaker question ('what is your favourite thing to do?'), $77 \%$ of participants referred to some form of exercise, irrespective of asthma status (81\% of those with asthma). Emergent themes are structured around activity type, highintensity interval training (HIIT), logistics and barriers (Figure 4.3).

All of the adolescents participated in some form of exercise; 38 in individual sports, 37 in team sports and 7 in dance. The participants identified five main categories of activity type as enjoyable, with team games widely stated as the most enjoyable (76\%). Due to its popularity, running was categorised separately to other individual sports, with suggestions that this activity type, particularly sprinting, was good to include given its simplicity. Obstacle courses and circuits were both mentioned as a way of implementing many different activities within the same session,

'because it's a range of things. People might find some easier than others and others might find it hard' G8.

Some of the participants with asthma $(n=4)$ raised the need for breaks within the exercise in order for them to catch their breath or,

“take your pump if you need to" B1.

High-intensity interval training was described by the majority as being hard work. Interestingly, the perceptions of HIIT difficulty were vastly different between those with and without asthma; only three of those with asthma thought that the difficulty would vary, with thirteen of those without asthma thinking that,

"it depends on what sort of exercise you do" B26.

In contrast to participants with asthma who perceived HIIT to be difficult specifically due to their asthma, their healthy counterparts attributed difficulty to a type of training their bodies are not used to,

“so like you're just instantly in something and it will be difficult” B20. 
Due to the structure of the school day, the adolescents were only able to participate in the exercise intervention out of school hours, resulting in a split for delivering the intervention before or after school, citing barriers such as after school activities. The majority of the participants would have preferred if the sessions were run outdoors (61\%), with $22 \%$ of participants asking for a combination of both indoor and outdoor activities, dependent on type of activity and weather.

A range of barriers to future exercise interventions were reported, such as illness or injury, or clashes with other activities, such as homework or paid work. The data revealed that $35 \%$ of the participants believed that those with asthma did not participate in as much exercise as their healthy peers, $48 \%$ perceived them to participate in the same amount and the remaining $17 \%$ believed that it was dependent on the activity in question. Lack of enjoyment of both team and individual sports was alluded to as one of the main barriers, contradicting previous activity choices. Running was specifically highlighted as a form of exercise that some participants without asthma found to be boring, and those with asthma reported difficulty breathing when running. In contrast to sprints, which were mentioned as an enjoyable activity type, long distance type running was cited as a barrier which the majority of asthma sufferers said they would find difficult. Being pushed too hard in the sessions was also mentioned as a barrier as it would decrease adherence to the intervention. 


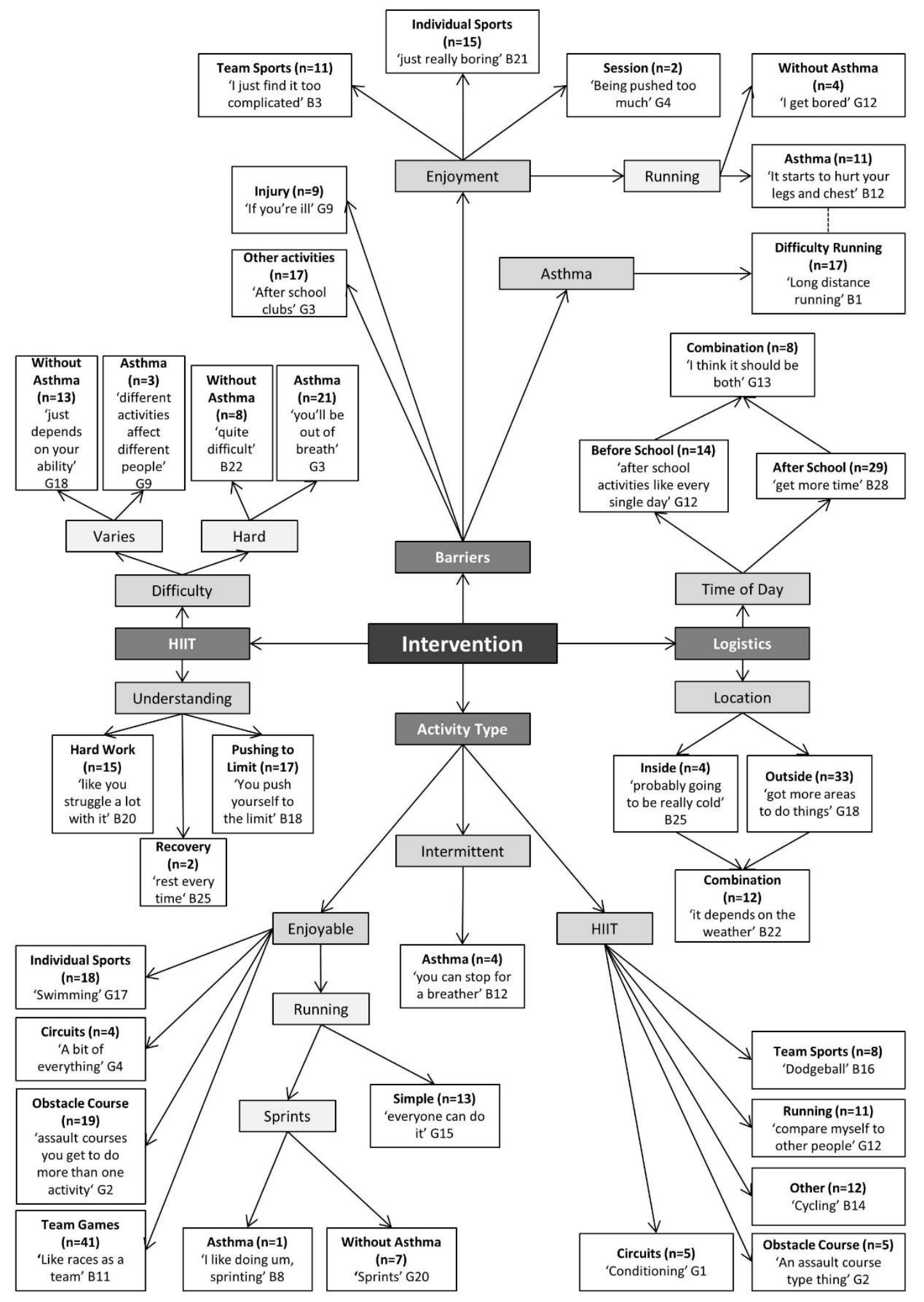

Figure 4.3. Pen profile of adolescents' perceptions of an exercise intervention

$\mathrm{B}=$ Boy $; \mathrm{G}=$ Girl $; \mathrm{PE}=$ Physical education 


\subsection{Discussion}

The primary aim of this study was to elicit perceptions about asthma and exercise from adolescents with and without the condition and to compare their perceived benefits and barriers to participation. Data were analysed thematically and presented using pen profiles, facilitating more accessible qualitative data for quantitative researchers and reducing the likelihood of skewed themes through dominating individuals in the interviews (Mackintosh et al., 2011). The second aim of the study was to inform the design, the content and delivery to co-develop the school-based, high-intensity interval training intervention (Chapter $6 \& 7$ ), the X4A trial: eXercise for Asthma with Commando Joe's, which was achieved using a representative sample across the ages within the planned intervention.

There are many known benefits of exercise for those who suffer from asthma (Mancuso et al., 2013, Philpott et al., 2010); however, only 23\% of participants with asthma and $18 \%$ of those without asthma were aware of the potential health benefits of exercise in asthma. Previous research documents the lack of knowledge of the benefits of exercise (Haughney et al., 2004) and therefore further education is required. Asthma guidelines highlight physical training as part of asthma management, with appropriate precautions for exercise-induced asthma (British Guideline on the Management of Asthma, 2008). Exercise reduces the symptoms and severity of asthma (Andrade et al., 2014) and, as lung function and wheeze in those with asthma are not adversely affected by exercise with appropriate treatment, there is no reason why they should not participate regularly (Ram et al., 2005).

Adolescents with asthma perceived that their healthy peers lacked understanding about the limiting effect of their condition on exercise, and that they use their condition as an excuse to be lazy. This perception of laziness and lack of understanding about asthma was confirmed by those without asthma. A minority of adolescents with asthma admitted to using their condition as an excuse, in contrast to previous research (Protudjer et al., 2012). These perceptions and misconceptions must be addressed through improved education about asthma and its implications for daily life and exercise. Indeed, two adolescents with asthma misjudged others with asthma, demonstrating a lack of understanding of their own condition. Only one 
person highlighted taking their inhaler prior to an activity as a control strategy suggesting a need for improved education to manage exercise-induced symptoms.

Exercise participation rates of those with and without asthma are considered to be conflicting, with suggestions that adolescents with asthma participate in more (Ownby et al., 2007, Weston et al., 1989), less (Lang et al., 2004, Taylor \& Newacheck, 1992) or equivalent levels of exercise to their healthy counterparts (Nystad, 1997, van Gent et al., 2007, Walders-Abramson et al., 2009). While the Chapter does not specifically address levels of exercise participation, adolescents with asthma were not perceived to engage in higher levels of exercise in comparison to their healthy peers, with most (48\%) stating that participation was similar or reduced $(35 \%)$. In an attempt to demonstrate that their condition does not negatively affect them, competitive motivation to outperform their healthy peers, was found to be a facilitator to $15 \%$ of adolescents with asthma, albeit more in terms of the intensity rather than volume of exercise (Trollvik et al., 2011). Participants without asthma discussed a wide range of barriers of asthma to exercise with relatively few facilitators, with fear of having an asthma attack identified as the main barrier by participants, irrespective of their asthma in agreement with a previous study (Williams et al., 2010). Despite fear of asthma attacks, $81 \%$ of those with asthma stated that their favourite activity was exercise mirroring previous findings of Fereday et al. (2009), demonstrating that these individuals overcome their fear in order to exercise.

Asthma has been previously found to impact on social (Nocon, 1991) and academic life (Tsakiris et al., 2013), often resulting in isolation (Trollvik et al., 2011). In contrast to previous studies where those with asthma reported being bullied or ignored due to their perceived limited physical capabilities (van den Bemt et al., 2010), adolescents with asthma in the present study did not report any form of bullying, although this was not directly asked. However, it is possible bullying would not have been raised in a group situation. School attendance was only discussed by two participants with asthma, although as suggested by van den Bemt et al., (2010) this may be due to the participants not perceiving absence from school as a negative factor; participants without asthma thought that poor school attendance may affect their upcoming exams, agreeing with previous findings (Nocon, 1991). 
As with previous research (Turner-Warwick, 1989), a lack of sleep due to asthma symptoms at night negatively impacted on subsequent social situations and concentration in school.

The adolescents reported participating in a variety of physical activities; the vast majority $(76 \%)$ liked the idea of team games, such as rounders, football or netball, which were commonly suggested by the participants, being incorporated into future interventions. Interestingly, there were no differences in type of activities suggested by those with and without asthma, with the exception of running, which was recommended by more adolescents without asthma. Almost half suggested obstacle courses or circuits to ensure a variety of different activities in each session, which might prevent burnout and boredom, and increase enjoyment during the sessions (Wilson et al., 2005). Indeed, this variation was suggested as a tool to divert attention away from the fear of an asthma attack and increase adherence to the exercise programme. Intermittent activities were suggested by four adolescents with asthma in order to catch their breath or take their medication. Given that it takes approximately 7.75 minutes of continuous exercise to elicit bronchoconstriction (van Leeuwen et al., 2011), intermittent activities may potentially reduce symptoms.

Participants with asthma generally thought that HIIT was difficult; this may be due to past experiences and potentially poor asthma control. Whilst asthma should not interfere with exercise if well controlled (Lucas \& Platts-Mills, 2005), congruent with previous research (van den Bemt et al., 2010), adolescents with asthma highlighted that their running ability was limited, impeding participation in the majority of sports. The current study, however, revealed that it was not running per se, but specifically long distance running that was difficult for those with asthma and therefore sprints could still be used within intervention sessions, also facilitating regular breaks.

Whilst the present findings significantly advance our understanding of the perceptions of those with and without asthma regarding exercise participation, and the ideal constituents for future interventions, it is perhaps pertinent to note the potential influence of self-selection bias on our findings. Indeed, as this study selected participants from those who assented to an exercise intervention study, they 
may not be a representative sample of the general adolescent population; for example, when asked what their favourite activity to participate in $(81 \%$ of participants stated exercise), the response was perhaps unusual in the age group. Such a self-selection bias would, however, be anticipated in any voluntary exercise intervention. Further work is required that focusses on the engagement of those with particularly low levels of physical activity. Furthermore, the positive outcomes of the present study may be under-represented as the participants in each group often tried to give answers that had not already been expressed. It is also important to highlight that the interviews were conducted in the summer and therefore answers to the preferred location of an intervention, time of day and types of sport may have reflected this. Contrary to previous research, parent interviews were not used in the present study as it was believed that it is the adolescents' engagement that is required for the sustainability of an intervention. Moreover, parents' perceptions of exercise and asthma have been found to be less accurate than their children with the condition (Panditi \& Silverman, 2003).

\subsection{Conclusion}

Educating adolescents about asthma could simultaneously aid in reducing stigmatisation and increasing the awareness of exercise-related health benefits, including better asthma control. Whilst participants with asthma reported a fear of undertaking exercise, it was still highlighted as their favourite activity, demonstrating promise for both future exercise interventions and the high-intensity interval training intervention of this thesis (Chapter 6 \& 7). Employing an inclusive exercise approach appears feasible given the similarity in activity choices between those with and without asthma. High-intensity, intermittent, varied exercise was highlighted as potentially effective at avoiding bronchoconstriction, distracting those with asthma from their preconceptions regarding exercise, yet ensuring enjoyment. 


\section{Thesis study map}

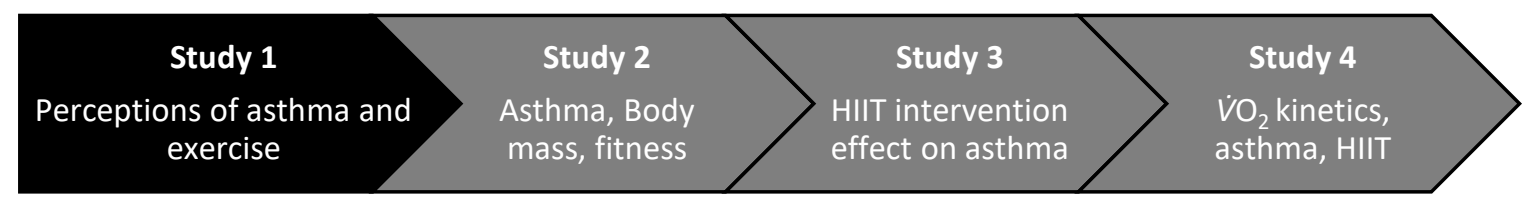

\section{Chapter 4}

Aims

- To elicit the views of adolescents, with and without asthma, about exercise and asthma, and the perceived benefits of, and barriers to, participation

- To inform the design of a high-intensity interval training intervention to improve asthma control

Key findings for future chapters

- Adolescents fear exercise although it is one of the favourite activities

- The intervention should be varied to avoid activity specific anxieties

- The intervention should be intermittent to allow those with asthma to "catch their breath or take their medication" 


\section{CHAPTER 5}

\section{INVESTIGATING THE RELATIONSHIP BETWEEN ASTHMA, BODY MASS AND AEROBIC FITNESS IN ADOLESCENTS}




\section{Chapter 5: Investigating the relationship between asthma, body mass and}

\section{aerobic fitness in adolescents}

This Study has been disseminated as follows:

Poster presentation: Winn CON, Mackintosh KA, Eddolls WTB, Stratton G, Wilson AM, McNarry MA, Davies GA (2015). The x4a study: the eXercise for asthma study. Swansea University Medical School Postgraduate conference; Swansea, UK

\subsection{Introduction}

The prevalence of asthma in the UK has dramatically risen over the last few decades (Asthma UK, 2017). This increase in asthma prevalence has been accompanied by a concurrent increase in obesity (Delgado et al., 2008, Townsend et al., 2013) and decrease in physical activity levels (Berntsen et al., 2016, Lang, 2014). Indeed, although the mechanisms and directionality remain unclear (McNarry et al., 2014a), recent studies have reported an association between asthma, obesity and fitness (Chen et al., 2013, McNarry et al., 2014a, Papoutsakis et al., 2013), with some studies even proposing a new phenotype of asthma related to a lack of cardiorespiratory fitness and/or physical activity. Indeed, as identified in Chapter 4 adolescents with asthma fear asthma attacks and consequently withdraw from exercise which could be related, at least in part, to a reduced cardiorespiratory fitness and physical activity.

Despite the importance of physical activity and exercise as tools to ameliorate asthma symptoms, current evidence regarding the fitness levels of those with asthma is equivocal, with some studies finding those with asthma to have poorer fitness (McNarry et al., 2014a, Vahlkvist \& Pedersen, 2009, Villa et al., 2011), whilst others show no difference between those with asthma and their healthy peers (Berntsen et al., 2009, Pianosi \& Davis, 2004, Santuz et al., 1997). These discrepancies may be due to the estimation of cardiorespiratory fitness from field-based measures which are subject to significant inaccuracies dependent on self-motivation and peer influence (Cairney et al., 2008). Moreover, even in studies which have utilised peak 
oxygen uptake $\left(\dot{V} \mathrm{O}_{2}\right)$ as a measure of cardiorespiratory fitness, the applicability of this measure to functional capacity during activities of daily living has been questioned (Jones, 2006, Matos \& Winsley, 2007). Indeed, differences between those with and without asthma may be detected using other parameters of aerobic fitness (Lucia et al., 2002). As asthma affects the airways, this may cause derangements in the $\mathrm{O}_{2}$ delivery, subsequently increasing the mean response time to exercise ( $\dot{V} \mathrm{O}_{2}$ kinetics). Moreover, if adolescents experience an increased $\mathrm{O}_{2}$ delivery does this potentially increase the total $\mathrm{O}_{2}$ cost of exercise $\left(\dot{V} \mathrm{O}_{2}\right.$ gain); these questions can be answered with more detailed analysis of the submaximal parameters of cardiorespiratory fitness which are currently lacking from the literature. Furthermore, whilst insufficient physical activity has been suggested to be causatively associated with asthma, physical activity has largely been assessed through self-report questionnaires, which are poorly correlated with objective measures (Tsai et al., 2012).

There is a strong positive correlation between cardiorespiratory fitness and physical activity among adolescents with asthma (Garfinkel et al., 1992). Moreover, there is a strong negative relationship between physical activity and obesity (WaldersAbramson et al., 2009). Each of these factors have previously been shown to be related to asthma occurrence, but no study to date has attempted to elucidate the relationships between asthma, fitness, physical activity and obesity.

Therefore, the aim of the present study was to investigate the influence of asthma on the submaximal and maximal parameters of cardiorespiratory fitness in adolescents. Furthermore, this study sought to further elucidate the potential relationship between cardiorespiratory fitness, physical activity, body mass and asthma.

\subsection{Methods}

\subsubsection{Participants}

Six-hundred and sixteen adolescents (334 boys; Table 5.1), of which 155 suffered from asthma (78 boys), from five schools across South Wales agreed to participate in the study. Ethical approval was granted by the institutional research ethics committee 
(ref: 140515 and PG/2014/29). Parent/guardian and head teacher consent and child assent were obtained prior to participation (see appendix A).

Table 5.1. Anthropometric measures for participants with and without asthma

\begin{tabular}{|c|c|c|c|c|c|c|c|c|}
\hline & \multirow{2}{*}{$\begin{array}{c}\text { n Asthma/Non- } \\
\text { asthma } \\
\text { (Boy,Girl) }\end{array}$} & \multicolumn{3}{|c|}{ Asthma } & \multicolumn{3}{|c|}{ Non-asthma } & \multirow{2}{*}{$\begin{array}{c}\text { Asthma v } \\
\text { Non-asthma } \\
\text { P-value }\end{array}$} \\
\hline & & Boy & Girl & $\begin{array}{c}\mathrm{P}- \\
\text { value }\end{array}$ & Boy & Girl & $\begin{array}{c}\mathrm{P}- \\
\text { value }\end{array}$ & \\
\hline Age (years) & $(78,77) /(256,201)$ & $13.0 \pm 1.2$ & $12.9 \pm 1.1$ & 0.71 & $13.1 \pm 1.1$ & $13 \pm 1.1$ & 0.25 & 0.45 \\
\hline Stature $(\mathrm{cm})$ & $(71,66) /(236,179)$ & $158.6 \pm 11.2$ & $154.3 \pm 7.7$ & $0.01 *$ & $158.5 \pm 10.7$ & $155.9 \pm 7.9$ & $0.01 *$ & 0.38 \\
\hline $\begin{array}{l}\text { Body mass } \\
(\mathrm{kg})\end{array}$ & $(71,65) /(236,179)$ & $58.3 \pm 16$ & $50.3 \pm 12.2$ & $<0.01 *$ & $51.6 \pm 13.3$ & $50.1 \pm 11.1$ & 0.23 & $0.01 *$ \\
\hline $\begin{array}{l}\text { Sitting stature } \\
(\mathrm{cm})\end{array}$ & $(71,66) /(236,179)$ & $79.4 \pm 5.7$ & $77.9 \pm 4.3$ & 0.09 & $79.2 \pm 5.7$ & $79.5 \pm 4.8$ & 0.53 & 0.11 \\
\hline $\begin{array}{l}\text { Waist } \\
\text { circumference } \\
(\mathrm{cm})\end{array}$ & $(71,64) /(236,178)$ & $77.4 \pm 12.7$ & $69.6 \pm 10.2$ & $<0.01 *$ & $70.4 \pm 12.7$ & $67 \pm 8.2$ & $<0.01 *$ & $<0.01 *$ \\
\hline $\begin{array}{l}\text { Age from } \\
\text { PHV (years) }\end{array}$ & $(71,65) /(236,179)$ & $-1.0 \pm 1.2$ & $0.6 \pm 0.9$ & $<0.01 *$ & $-1.0 \pm 1.1$ & $0.7 \pm 1.0$ & $<0.01 *$ & 0.58 \\
\hline BMI $\left(\mathrm{kg} \cdot \mathrm{m}^{-2}\right)$ & $(71,65) /(236,179)$ & $23 \pm 4.7$ & $21.4 \pm 4.8$ & 0.05 & $20.4 \pm 3.9$ & $20.5 \pm 3.5$ & 0.8 & $<0.01 *$ \\
\hline
\end{tabular}

$*=$ Significant $\mathrm{p}<0.05 ; \mathrm{PHV}=$ Peak height velocity $; \mathrm{BMI}=$ Body mass index.

\subsubsection{Study design}

This experimental chapter describes an observational case-controlled study designed to test whether there were any differences in fitness, obesity, physical activity, lung function and quality of life between adolescents with and without asthma. In addition, it examines the association between asthma, obesity, physical activity and fitness. 


\subsubsection{Procedures}

\subsubsection{Anthropometrics}

Body mass and stature were measured according to the techniques outlined by International Society for the Advancement of Kinanthropometry (Stewart et al., 2011). Stature, sitting stature and waist circumference were measured to the nearest $0.01 \mathrm{~m}$ (Seca213, Hamburg, Germany) and body mass to the nearest $0.1 \mathrm{~kg}$ (Seca876, Hamburg, Germany). Body mass index (BMI) was subsequently calculated and grouped using age and sex specific child percentiles (Barlow, 2007). Further, lower limb length was calculated as the difference between stature and sitting stature and then used to determine maturity offset using the equations of Mirwald et al. (2002).

\subsubsection{Physical activity}

Physical activity levels were measured at $100 \mathrm{~Hz}$ using ActiGraph GT3X+ accelerometers (Actigraph, Pensacola, FL, USA) worn on the right hip for seven consecutive days. Participants were instructed only to remove the monitor it if they undertook water-based or contact activities, where required. The data were analysed using KineSoft version 3.3.67 (KineSoft, Saskatchewan, Canada) employing 1 second epochs with sustained periods of at least 20-minutes of consecutive zeros considered non-wear time (Catellier et al., 2005). A minimum daily wear time of 10 hours per day for 2 weekdays and 1 weekend day was used (Rich et al., 2013). Physical activity intensities were calculated using Evenson et al. (2008) cut points, which have been shown to be valid and reliable determinats of activity intensity in children and adolescents (Trost et al., 2011).

\subsubsection{Lung function}

Forced Expiratory Volume in 1 second $\left(\mathrm{FEV}_{1}\right)$, Forced Vital Capacity (FVC), $\mathrm{FEV}_{1} / \mathrm{FVC}$ ratio, Peak Expiratory Flow (PEF), and Forced Expiratory Flow between $25-75 \%$ of vital capacity $\left(\mathrm{FEF}_{25-75}\right)$ was measured using a portable dry spirometer (Vitalograph, Buckingham, UK). The best of three measurements were taken according to American Thoracic Society guidelines (1995) and to the standardised 
protocol (Miller et al., 2005) and expressed as a percentage of the age-sex-stature predicted value (Rosenthal et al., 1993).

\subsubsection{Fractional Exhaled Nitric Oxide}

Participants with asthma were asked to perform a Fractional Exhaled Nitric Oxide (FeNO) test, a marker of airway inflammation in asthma, prior to spirometric testing. The FeNO test was performed in a seated position and in accordance with the American Thoracic Society guidelines (Dweik et al., 2011). Participants were asked to exhale away from the device (NIOX MINO, Aerocrine AB, Solna, Sweden) and then inhale to total lung capacity through the device before immediately exhaling for 10 seconds at $50 \pm 5 \mathrm{ml} \cdot \mathrm{sec}^{-1}$. Visual and audio cues were provided by the computer software throughout. One test was completed and the final three seconds of exhalation were evaluated.

\subsubsection{Asthma control}

Asthma control was measured using the Asthma Control Questionnaire (ACQ, Juniper et al., 2010) which consists of 7-items relating to recent symptoms, medications and $\mathrm{FEV}_{1}$ score. Each item of the ACQ was scored from 0 to 6 and then averaged to give an overall result. Scores of $\leq 0.75$ or $\geq 1.5$ indicated well-controlled and poorly-controlled asthma, respectively. Internal consistency, measured using Cronbach's alpha coefficients (Cronbach, 1951), for the ACQ were deemed acceptable $(\alpha=0.77)$.

\subsubsection{Asthma-related quality of life}

The Paediatric Asthma Quality of Life Questionnaire (PAQLQ) was used to assess the symptoms, activity limitations and emotional and environmental effects of asthma (Juniper et al., 1996). The PAQLQ consists of 23 questions (scored on a Likert scale from 1 to 7 ), with a higher score indicating a better asthma status. Internal reliability for the PAQLQ was deemed excellent $(\alpha=0.97)$. 


\subsubsection{Quality of life}

The Pediatric Quality of Life Inventory (PedsQL) Teenager Report (Version 4.0, Varni et al., 1999) was used to compare the perceived quality of life between those participants with and without asthma. A widely validated measure in adolescents (Varni et al., 2006, Varni et al., 2003, Varni et al., 2001), the PedsQL consists of 23 items focusing on participants' physical, emotional, social and school functioning quality, with a higher score indicative of a better quality of life. Internal reliability for the PedsQL was excellent $(\alpha=0.90)$.

\subsubsection{Asthma severity}

Asthma severity was assessed using the Global Initiative for Asthma guidelines (Global Initiative for Asthma, 2017) and classified as mild, moderate or severe according to the medication step required to achieve asthma control. For the purpose of analysis, moderate and severe asthma were grouped to power the statistics. Participants were excluded if they did not have stable asthma $(n=4)$.

\subsubsection{Cardiorespiratory fitness}

Participants were asked to refrain from strenuous exercise and avoid consuming food for $24 \mathrm{~h}$ and $2 \mathrm{~h}$ prior to the exercise test, respectively.

20-metre shuttle run

Cardiorespiratory fitness was estimated using the 20-metre progressive shuttle run test, a previously validated field measure in children (Mayorga-Vega et al., 2015). The number of shuttles completed before voluntary exhaustion were recorded.

\section{Peak $\dot{V} \mathrm{O}_{2}$}

Sixty-nine adolescents (39 boys) inclusive of 36 with asthma (21 boys) were selected, using stratified randomisation, to complete incremental ramp tests. The groups were stratified by age, sex and asthma to provide a representative sample of the wider population. Participants performed an incremental ramp exercise test to volitional exhaustion on an electromagnetically braked cycle ergometer (Ergoselect 200, Ergoline GmbH, Lindenstrasse, Germany). The ramp protocol consisted of 3 
minutes of "unloaded" pedalling $(0 \mathrm{~W})$ followed by an increase in work rate of 12-24 $\mathrm{W} \cdot \mathrm{min}^{-1}$ dependent on pre-baseline familiarisation incremental ramp tests. Throughout the test, participants were asked to keep the cadence at $75 \pm 5$ revolutions per minute. Pulmonary ventilation (VE) and gas exchange $\left(\dot{V} \mathrm{O}_{2}\right.$ and $\dot{V} \mathrm{CO}_{2}$ ) were measured breath by breath (Jaeger Oxycon Mobile, Jaeger, Hoechberg, Germany).

\subsubsection{Data analysis}

The peak $\dot{V} \mathrm{O}_{2}$ was taken as the highest 10 -second average value attained prior to the end of the test with the GET determined using the V-slope method (Beaver et al., 1986). Analysis of covariance (ANCOVA) was used to account for the influence of body size using log transformed data to determine the allometric relationship between peak $\dot{V} \mathrm{O}_{2}$ and body mass. Common allometric exponents were confirmed for the data and power function ratios $\left(\mathrm{Y} / \mathrm{X}^{\mathrm{b}}\right)$ were computed. Breath by breath data were then averaged into 10 -second time bins and the gain and mean response time (MRT) calculated according to the methods reported by Barstow et al. (2000b). Specifically, the gain $\left(\Delta \dot{V} \mathrm{O}_{2} / \Delta \mathrm{WR}\right)$ was determined by linear regression over three segments: $S_{1}$, from 1-minute into the ramp test up to GET; $S_{2}$, from GET to peak $\dot{V} \mathrm{O}_{2}$; and $\mathrm{S}_{\mathrm{T}}$, over the range of $\mathrm{S}_{1}+\mathrm{S}_{2}$. The MRT was calculated as the point of intersection between the baseline $\dot{V} \mathrm{O}_{2}$ and a backwards linear extrapolation of the $\dot{V} \mathrm{O}_{2}$ by time slope from the onset of the increase in work rate (Glantz, 1990). The MRT was also determined using two segments, $\mathrm{S}_{1}\left(\mathrm{MRT}_{1}\right)$ and $\mathrm{S}_{\mathrm{T}}$ (MRTT, Whipp et al., 1981). Baseline $\dot{V} \mathrm{O}_{2}$ was taken as the average of the first 45 seconds of the last minute prior to the increase in work rate.

\subsubsection{Statistical analysis}

Shapiro-Wilk tests were used to examine the normality of the data prior to any analyses. In the case of normally distributed data, independent sample t-tests were used to assess differences between participants with and without asthma. A MannWhitney $U$ test was used when data were not normally distributed. Analysis of covariance was used to investigate the influence of asthma on cardiorespiratory fitness and its interaction with sex, age and maturity. One-way ANOVA tests were also used to determine the influence of the level of asthma severity. Pearson's 
correlation coefficients were used to investigate the degree of association between key variables. Furthermore, the association between asthma and BMI was assessed by binary logistic regression adjusting for fitness and time spent in moderate-tovigorous physical activity (MVPA). Missing data were imputed using multiple imputation for physical activity data, this was done using all other measures for each participant to predict the missing value. All statistical analyses were conducted using SPSS v22 (IBM Corp, Armonk, NY). All data are presented as mean \pm standard deviation (SD) with statistical significance accepted as $\mathrm{P}<0.05$.

\subsection{Results}

Those with asthma were predominantly characterised as having mild, persistent asthma $(85 \%)$, with the minority having moderate or severe asthma (15\%, Global Initiative for Asthma, 2017). As shown in Table 5.1, no anthropometric differences were shown between those with and without asthma, with the exception of body mass and waist circumference which were significantly higher in those with asthma. Similarly, those with asthma had a significantly $(\mathrm{P}<0.01)$ higher BMI $(22.2 \pm 4.8$ $\left.\mathrm{kg} \cdot \mathrm{m}^{-2}\right)$ than their healthy peers $\left(20.4 \pm 3.7 \mathrm{~kg} \cdot \mathrm{m}^{-2}\right)$. Age-specific BMI percentiles revealed $41.9 \%$ of participants with asthma were overweight or obese, in comparison to $25.4 \%$ of healthy participants. Participants with asthma spent significantly ( $\mathrm{P}<$ $0.05)$ less time in both moderate $(31.1 \pm 11.7$ minutes $)$ and vigorous $(22.9 \pm 13.2$ minutes) physical activity than their healthy peers $(34.6 \pm 13.1$ and $25.9 \pm 13.2$, respectively). A lower $\mathrm{FEV}_{1} \%$ and more marked small airways obstruction ( $\mathrm{FEF}_{25}$ ${ }_{75} \%$ ) was observed in those with asthma. However, those with asthma did not have an obstructed $\mathrm{FEV}_{1} / \mathrm{FVC}$ ratio, consistent with most having mild asthma.

As shown in Table 5.2, the ACQ revealed 32\% of participants had well-controlled asthma (score $<0.75$ ), 36\% had intermediate control and the remaining $32 \%$ had poorly controlled asthma (score >1.5). According to the PAQLQ, 14\% of participants with asthma reported a score less than 4, with 5\% scoring 7. Although the mean of the PAQLQ is relatively high, 95\% scored less than 7 indicating at least some degree of impairment. 
In contrast to the 20-metre shuttle run in which healthy participants completed significantly more shuttles than those with asthma (48 \pm 24 vs $42 \pm 23$ shuttles, respectively; $\mathrm{P}<0.01$ ), peak $\dot{V} \mathrm{O}_{2}$ and scaled peak $\dot{V} \mathrm{O}_{2}$ did not differ according to asthma status (Table 5.3). All participants achieved a rating of perceived exertion $>18$, and a heart rate greater than $85 \%$ age predicted heart rate maximum, however, one participant failed to reach a respiratory exchange ratio $>1.0$. Similarly, there were no significant differences between asthma and non-asthma groups in the absolute or relative GET or the gain. However, participants with asthma did have a significantly shorter $\mathrm{MRT}_{\mathrm{T}}$, although these differences were not observed below the GET and were ameliorated once work rate was added as a covariate.

Although significant differences were shown between girls and boys for cardiorespiratory fitness and physical activity levels, sex did not account for any of the variance between asthma and non-asthma groups. Therefore, boys and girls were pooled for all subsequent analyses. The number of shuttles completed in the 20metre shuttle run was negatively correlated with $\mathrm{BMI}(\mathrm{r}=-0.34, \mathrm{P}<0.05)$ and positively associated with MVPA $(r=0.34, \mathrm{P}<0.05)$ pooled for both those with and without asthma. Body mass index was also negatively associated with MVPA ( $\mathrm{r}=$ $0.18, \mathrm{P}<0.05)$. Positive associations were shown between cardiorespiratory fitness according to both the 20-metre shuttle run and peak $\dot{V} \mathrm{O}_{2}$ and ACQ $(\mathrm{r}=-0.15$ and 0.35; $\mathrm{P}<0.05)$, PAQLQ $(\mathrm{r}=0.27$ and $0.34, \mathrm{P}<0.05)$ and PedsQL $(\mathrm{r}=0.22$ and $0.35, \mathrm{P}<0.05)$. Furthermore, participants without asthma reported a significantly higher quality of life $(78.2 \pm 14.7$ vs $74.4 \pm 17.8)$. Whilst significant, these correlations are weak and should be interpreted with caution.

As BMI, 20-metre shuttle run and MVPA were significantly different between the asthma and non-asthma groups, individual logistic regression analyses were performed on each. Both BMI and fitness were shown to be significantly associated with asthma; MVPA failed to reach significance $(\mathrm{p}=0.06)$ even when using multiple imputation to replace missing MVPA data. Binary logistic regression (Table 5.4) revealed BMI as an independent factor associated with asthma. 
Table 5.2. Characteristics of participants with and without asthma

\begin{tabular}{|c|c|c|c|c|}
\hline & $\begin{array}{c}\mathrm{n} \text { (Asthma / } \\
\text { Non-asthma) }\end{array}$ & Asthma & Non-asthma & P-value \\
\hline $\begin{array}{l}\text { 20-metre shuttle run } \\
\text { (shuttles) }\end{array}$ & $138 / 395$ & $42.1 \pm 22.9$ & $47.7 \pm 24.1$ & $0.01 *$ \\
\hline MPA (min) & $61 / 177$ & $31.1 \pm 11.7$ & $34.6 \pm 13.1$ & $0.03^{*}$ \\
\hline VPA (min) & $61 / 177$ & $22.9 \pm 13.2$ & $25.9 \pm 13.2$ & $0.04^{*}$ \\
\hline MVPA (min) & $61 / 177$ & $53.9 \pm 23.5$ & $60.5 \pm 23.6$ & $0.02 *$ \\
\hline $\mathrm{FEV}_{1}(\%$ Predicted $)$ & $143 / 411$ & $91.0 \pm 14.7$ & $95.7 \pm 16.4$ & $<0.01^{*}$ \\
\hline FVC (\%Predicted) & $143 / 411$ & $99.0 \pm 14.2$ & $99.7 \pm 14.1$ & 0.57 \\
\hline $\mathrm{FEF}_{25-75}(\%$ Predicted $)$ & $143 / 411$ & $76.0 \pm 25.3$ & $87.0 \pm 24.5$ & $<0.01 *$ \\
\hline PEF (\%Predicted) & $143 / 411$ & $91.2 \pm 19.6$ & $89.8 \pm 20.2$ & 0.47 \\
\hline $\mathrm{FEV}_{1} / \mathrm{FVC}$ & $143 / 411$ & $0.80 \pm 0.08$ & $0.83 \pm 0.07$ & $<0.01 *$ \\
\hline FeNO (ppb) & $139 / 0$ & $42.7 \pm 44.0$ & NA & NA \\
\hline PedsQL PhH & $132 / 400$ & $76.3 \pm 18.3$ & $80.3 \pm 14.6$ & 0.10 \\
\hline PedsQL PsH & $132 / 400$ & $73.3 \pm 19.4$ & $77.2 \pm 16.5$ & 0.06 \\
\hline PedsQL Total & $132 / 400$ & $74.4 \pm 17.8$ & $78.2 \pm 14.7$ & $0.04 *$ \\
\hline ACQ & $143 / 0$ & $1.3 \pm 1.0$ & NA & NA \\
\hline PAQLQ Symptoms & $143 / 0$ & $5.5 \pm 1.2$ & NA & NA \\
\hline PAQLQ Activities & $143 / 0$ & $5.5 \pm 1.2$ & NA & NA \\
\hline PAQLQ Emotions & $143 / 0$ & $5.9 \pm 1.3$ & NA & NA \\
\hline PAQLQ & $143 / 0$ & $5.6 \pm 1.2$ & NA & NA \\
\hline \multicolumn{5}{|c|}{$\begin{array}{l}\text { Mean } \pm \text { SD } ; * \text { Significant } \mathrm{p}<0.05 ; \mathrm{NA}=\text { Not applicable; MPA = moderate } \\
\text { physical activity; VPA = Vigorous physical activity; MVPA = Moderate-to- } \\
\text { vigorous physical activity; \% Predicted = Expressed as a percentage of the age- } \\
\text { sex-stature predicted value; } \mathrm{FEV}_{1}=\text { Forced Expiratory Volume in } 1 \text { second; } \\
\text { FVC = Forced Vital Capacity; FEF } 25-75=\text { Forced Expiratory Flow between } 25- \\
75 \% \text { of vital capacity; PEF = Peak Expiratory Flow; FeNO = Fractional exhaled } \\
\text { nitric oxide; PedsQL = Pediatric Quality of Life Inventory; PhH = Physical } \\
\text { score; PsH = Psychological score; ACQ = Asthma control questionnaire; } \\
\text { PAQLA = Paediatric Asthma Quality of Life Questionnaire }\end{array}$} \\
\hline
\end{tabular}


Table 5.3. Incremental ramp test results for participants with and without asthma

\begin{tabular}{|c|c|c|c|}
\hline & Asthma & Non-asthma & P-value \\
\hline Baseline $\dot{V} \mathrm{O}_{2}\left(1 \cdot \min ^{-1}\right)$ & $0.60 \pm 0.10$ & $0.60 \pm 0.10$ & 0.70 \\
\hline$\dot{V} \mathrm{O}_{2}$ peak $\left(1 \cdot \min ^{-1}\right)$ & $2.00 \pm 0.50$ & $2.10 \pm 0.50$ & 0.67 \\
\hline$\dot{V} \mathrm{O}_{2} \mathrm{BM}\left(\mathrm{ml} \cdot \mathrm{kg}^{-1} \cdot \mathrm{min}^{-1}\right)$ & $36.9 \pm 8.3$ & $41.1 \pm 7.4$ & $0.02 *$ \\
\hline$\dot{V} \mathrm{O}_{2}$ Scaled $\left(\mathrm{ml} \cdot \mathrm{kg}^{-0.57} \cdot \mathrm{min}^{-1}\right)$ & $205.1 \pm 43.6$ & $221.6 \pm 38.6$ & 0.11 \\
\hline $\operatorname{GET}\left(1 \cdot \min ^{-1}\right)$ & $1.10 \pm 0.30$ & $1.00 \pm 0.30$ & 0.62 \\
\hline $\operatorname{GET}\left(\% \dot{V} \mathrm{O}_{2}\right)$ & $53.8 \pm 7.9$ & $50.5 \pm 6.2$ & 0.06 \\
\hline $\operatorname{MRT}_{\mathrm{T}}(\mathrm{s})$ & $72.5 \pm 17.5$ & $81.5 \pm 17.7$ & $0.04 *$ \\
\hline $\mathrm{MRT}_{1}(\mathrm{~s})$ & $69.6 \pm 13.6$ & $75.5 \pm 17.0$ & 0.12 \\
\hline $\mathrm{S}_{1}$ Gain $\left(\mathrm{ml} \cdot \mathrm{min}^{-1} \cdot \mathrm{W}^{-1}\right)$ & $9.0 \pm 1.8$ & $9.1 \pm 2.8$ & 0.78 \\
\hline $\mathrm{S}_{2}$ Gain $\left(\mathrm{ml} \cdot \mathrm{min}^{-1} \cdot \mathrm{W}^{-1}\right)$ & $10.8 \pm 2.0$ & $11.0 \pm 1.3$ & 0.55 \\
\hline $\mathrm{S}_{\mathrm{T}}$ Gain $\left(\mathrm{ml} \cdot \mathrm{min}^{-1} \cdot \mathrm{W}^{-1}\right)$ & $10.0 \pm 1.5$ & $10.3 \pm 1.4$ & 0.49 \\
\hline \multicolumn{4}{|c|}{$\begin{array}{l}\text { Mean } \pm \mathrm{SD} ; * \text { = Significant } \mathrm{p}<0.05 ; \mathrm{NA}=\text { Not applicable; } \dot{\mathrm{V} O} 2=\text { Oxygen } \\
\text { uptake; } \mathrm{BM}=\text { body mass; GET }=\text { Gas exchange threshold; } \mathrm{S}_{1} \text { gain }=\text { Gain from } \\
\text { 1-minute into ramp to GET; } \mathrm{S}_{2} \text { gain }=\text { Gain from GET to peak } \mathrm{V} O 2 ; \mathrm{S}_{\mathrm{T}} \text { gain }= \\
\text { Gain over the total range } \mathrm{S} 1+\mathrm{S} 2 ; \mathrm{MRT}_{1}=\text { Mean response time for } \mathrm{S}_{1} ; \mathrm{MRT}_{\mathrm{T}}= \\
\text { Mean response time for } \mathrm{S}_{1}+\mathrm{S}_{2}\end{array}$} \\
\hline
\end{tabular}

Table 5.4. Binary logistic regression analysis to test potential association with asthma from Body Mass Index, Moderate-toVigorous Physical Activity and 20-metre shuttle run

\begin{tabular}{|c|c|c|c|c|}
\hline & $\beta(\mathrm{SE})$ & Wald & P-value & OR \\
\hline Constant & $2.24(0.72)$ & 9.97 & $<0.01$ & 9.41 \\
\hline BMI & $-0.09(0.02)$ & 12.89 & $<0.01$ & 0.92 \\
\hline MVPA & $0.01(0.01)$ & 1.09 & 0.28 & 1.01 \\
\hline Shuttle Run & $0.01(0.00)$ & 2.18 & 0.19 & 1.01 \\
\hline
\end{tabular}




\subsection{Discussion}

The present study highlights the importance of the measure of cardiorespiratory fitness used when investigating the influence of asthma in adolescents. Specifically, contrary to the findings reported here and elsewhere with regards to the 20-metre shuttle run, when more accurate and sensitive measures of cardiorespiratory fitness are used, there was no difference between those with and without asthma. Furthermore, the present study reveals obesity to be a significant predictor of asthma status and those with asthma to engage in less MVPA. Taken together, these findings highlight important potential targets for future interventions that seek to reduce asthma severity and prevalence.

The current participants with asthma reported a lower quality of life than their healthy counterparts, in agreement with previous studies (Merikallio et al., 2005, Molzon et al., 2013), although it is pertinent to note that the majority were characterised by poor asthma control which is likely to have reduced their quality of life over and above the effects of asthma per se (Sundbom et al., 2016). Interestingly, only fitness was shown to be related to quality of life and asthma control, although this was a weak correlation, fitness could possibly represent a key target to improve quality of life in those with asthma as observed in previous studies (Andersen et al. 2017, Basaran et al., 2006, Fanelli et al., 2007). Such improvements in fitness may be elicited through improvements in BMI and physical activity which were associated with fitness in the current study. Indeed, those with asthma not only demonstrated a higher BMI, in accord with previous studies (Black et al., 2012, McNarry et al., 2014a), but also a significantly lower MVPA (Sousa et al., 2014, Villa et al., 2011), with the majority of those with asthma failing to meet the recommended guidelines of 60-minutes MVPA per day (Department of Health, 2011). These low MVPA levels may be attributable to a fear of asthma attack associated with exercise as shown in Chapter 4 but, contrastingly, exercise-related activities were still cited as the most enjoyable activity by over $80 \%$ of adolescents with asthma.

The current cardiorespiratory fitness results significantly differed according to their method of determination. Specifically, according to the 20-metre shuttle run, those 
with asthma were significantly less fit than their healthy counterparts but both groups demonstrated a relatively high degree of fitness relative to recently generated receiver operating characteristic cut-points (Boddy et al., 2012). In contrast, using the gold standard measure of cardiorespiratory fitness of peak $\dot{V} \mathrm{O}_{2}$ (Carey \& Richardson, 2003), those with asthma were comparable to those without asthma. These results were also comparable to previous research $\left(37-41 \mathrm{ml} \cdot \mathrm{min}^{-1} \cdot \mathrm{kg}^{-1} \mathrm{vs}\right.$. 35 $48 \mathrm{ml} \cdot \mathrm{min}^{-1} \cdot \mathrm{kg}^{-1}$, Armstrong, 2006); although slightly lower which is likely attributable to the use of a cycle ergometer as oxygen consumption is approximately 7\% higher when using a treadmill (Shepherd, 1971). This discrepancy could be due to self-perceptions and peer-perceptions of (in)ability (Chapter 4), causing those with asthma to limit their performance in front of others compared to when tested in isolation or on a non-familiar modality (Glazebrook et al., 2006, Lang et al., 2004). It could also be suggested that such perceptions may be exacerbated by the greater BMI of those with asthma, as it is frequently cited that those who are overweight are unwilling to exercise in front of their peers (Ball et al., 2000). Finally, participation in the incremental ramp test may have resulted in a greater self-selection bias, as this was a much more extensive measure, and therefore the participants with lower cardiorespiratory fitness may have opted out, accounting for the disparity between measures. The lack of effect of asthma on peak $\dot{V} \mathrm{O}_{2}$ in the present study agrees with previous studies (Berntsen et al., 2009, Pianosi \& Davis, 2004, Santuz et al., 1997) and suggests that previous studies utilising indirect estimates of cardiorespiratory fitness may have drawn erroneous conclusions (Cairney et al., 2008, McNarry et al., 2014a), as would also have been done according to the current 20-metre shuttle run results. The relatively low peak $\dot{V} \mathrm{O}_{2}$ values (Armstrong et al., 1996, Rodrigues et al., 2006) reported in this study nonetheless highlight an area of concern with regards to the current health of adolescents (Ortega et al., 2008). Indeed, considering that peak $\dot{V} \mathrm{O}_{2}$ is one of the strongest predictors of all-cause mortality (Blair et al., 1989, Kodama et al., 2009), with a strong relationship between peak $\dot{V} \mathrm{O}_{2}$ as a child and adult (Malina, 2001), the current results highlight the need for interventions that successfully, and sustainably, increase the cardiorespiratory fitness of youth.

This is the first study to consider the influence of asthma on the sub-maximal parameters of aerobic fitness (MRT, gain and GET), many of which have been 
suggested to be more sensitive to both advantageous and deleterious adaptations than peak $\dot{V} \mathrm{O}_{2}$ (McNarry et al., 2017). However, in agreement with peak $\dot{V} \mathrm{O}_{2}$, no influence of asthma was manifest on any parameter of aerobic fitness. Whilst other respiratory diseases have been found to be associated with significant differences in gain between those with and without the condition (Fielding et al., 2015), participants with asthma were not different to their peers. These findings suggest that adolescents with asthma do not engender a greater $\mathrm{O}_{2}$ cost of exercise in comparison to their peers. The findings on the GET suggest that those with asthma are able to participate in similar training programmes as those without. Although optimal training should be based on an individuals' GET, as differences were not shown, those with asthma will have comparable training "zones" that should elicit similar improvements. These findings are in accord with previous research showing no differences between asthma and their healthy counterparts (Santuz et al., 1997). Whilst the GET was low in comparison to previous findings (Fawkner \& Armstrong, $2004 \mathrm{a})$, the results do not suggest deconditioning $\left(<50 \%\right.$ peak $\dot{V} \mathrm{O}_{2}$, Urquhart \& Vendrusculo, 2017). In contrast, it is perhaps interesting to note the significantly longer MRT found here than in previously reported research (Barstow et al., 2000a, McNarry et al., 2011b), which may suggest chronic deconditioning and agreeing with the relatively low peak $\dot{V} \mathrm{O}_{2}$ values observed from the cycle ergometer. These findings must be interpreted with caution however due to the influence of ramp rate on the MRT which limits inter-study comparisons (Boone et al., 2008). The lack of a difference between adolescents with and without asthma in peak $\dot{V} \mathrm{O}_{2}$ and subsequently the MRT suggests that any derangements in airways of the participants with asthma do not affect the $\mathrm{O}_{2}$ delivery to the mitochondria within the muscle.

In agreement with previous studies (McNarry et al., 2014a, Vahlkvist et al., 2010), the prevalence of overweight and obesity in the present participants with asthma was high in comparison to their peers. Whilst the causal relationship between asthma and obesity remains unclear, postulated mechanisms include co-morbidities or mechanical effects of an increased pressure caused by excess tissue mass in the abdomen and chest influencing hyper-responsiveness or symptoms of asthma directly (Farah \& Salome, 2012). Alternatively, or additionally, the increased BMI in those with asthma could be related to the over-diagnosis of asthma in obese people 
(van Huisstede et al., 2013), with obesity significantly influencing many spirometric parameters (Spathopoulos et al., 2009).

There was a large range of FeNO score with the mean $(42.7 \pm 44.0 \mathrm{ppb})$ considered high (children $>35 \mathrm{ppb}$, adults $>50$, Dweik et al., 2011). The current FeNO scores were also higher than reported elsewhere in well-controlled asthma (Willeboordse et al., 2016), indicating sub-optimal control of airway inflammation and raising the possibility of poor inhaler technique and/or poor medication adherence. This is a significant problem, especially in youth with asthma who cite barriers to physical activity such as administering medication in front of their peers and embarrassment of their condition (Cohen et al., 2003). When reporting their medication and adherence, participants often described taking their prescribed preventer sporadically and not as directed (Chapman et al., 2017). This poor control is likely to exacerbate their symptoms during exercise, further reinforcing their perception of an inability to, and fear of, exercise. Therefore, a potential solution to their lack of fitness and physical activity and increased BMI could be as simple as education on proper inhaler technique.

A major strength of this study was the use of more sensitive parameters of aerobic fitness (GET, MRT and gain) which have not been previously assessed in adolescents with asthma. Furthermore the use of objective measures of physical activity should also be considered a strength of the study. In addition, there may have been a self-selection bias such that participants with more severe asthma and/or poorer fitness may have chosen to opt out of more vigorous sub-sample testing. Furthermore, as asthma medication was not withheld prior to, or during the exercise measures, this may have effectively "normalised" the participants results in comparison to their healthy peers. Finally, the different modalities of the field and lab-based measures of cardiorespiratory fitness limits our interpretation to some extent. 


\subsection{Conclusion}

In conclusion, adolescents with predominantly mild persistent asthma do not differ in cardiorespiratory fitness from their peers, however, they do have an increased BMI and engage in less MVPA. The present findings also highlight the importance of using appropriate measures of cardiorespiratory fitness to determine the influence of disease on exercise responses. Finally, although only a weak relationship was found between cardiorespiratory fitness and quality of life, further studies should investigate if cardiorespiratory fitness can reduce asthma severity in adolescents with more severe asthma. 


\section{Thesis study map}

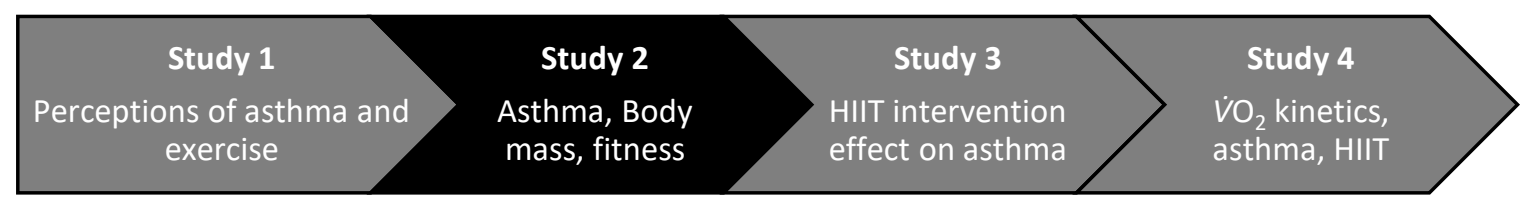

\section{Chapter 5}

Aims

- To investigate the influence of asthma on the submaximal and maximal parameters of cardiorespiratory fitness in adolescents

- To elucidate the potential relationship between cardiorespiratory fitness, physical activity, body mass and asthma

Key findings for future chapters

- Adolescents with or without asthma do not differ in cardiorespiratory fitness

- Those with asthma do participate in significantly less moderate-to-vigorous physical activity and have higher body mass index than their healthy peers

- More precise measures of cardiorespiratory fitness should be utilised to determine the influence of disease on the cardiorespiratory system 


\title{
CHAPTER 6
}

\author{
EFFECT OF HIGH-INTENSITY \\ INTERVAL TRAINING IN \\ ADOLESCENTS WITH ASTHMA
}




\section{Chapter 6: Effect of high-intensity interval training in adolescents with asthma}

This Study has been disseminated as follows:

Oral presentation: Winn CON, Mackintosh KA, Eddolls WTB, Stratton G, Wilson AM, Davies GA, McNarry MA (2016). The effects of a six-month high-intensity interval training intervention on aerobic fitness and fatness in adolescents with asthma. Asthma UK Centre for Applied Research joint centre event London UK

Oral presentation: Winn CON, Mackintosh KA, Eddolls WTB, Stratton G, Wilson AM, Davies GA, McNarry MA (2016). Effect of high-intensity exercise on aerobic performance and airway inflammation in asthma. European Respiratory Society International Congress; London UK

\subsection{Introduction}

The prevalence of asthma and obesity have both risen dramatically over the past few decades, making them two of the most common chronic conditions in the UK ( $\mathrm{Ng}$ et al., 2014, Wanrooij et al., 2014). This concomitant rise has led to suggestions that the two may be causatively linked (Farah \& Salome, 2012, Lucas \& Platts-Mills, 2006), with overweight and obesity more prevalent in those who suffer from asthma (Vahlkvist \& Pedersen, 2009).

It has been postulated that an influential factor in the relationship between asthma and obesity is cardiorespiratory fitness (Lochte et al., 2009), with previous research noting a strong inverse relationship between maximum oxygen uptake $\left(\dot{V} \mathrm{O}_{2}\right)$ and indices of adiposity in young adults (Hingorjo et al., 2017). However, it remains to be elucidated as to whether the role of cardiorespiratory fitness is direct, a mediator of the influence of obesity or, potentially, both. Indeed, the influence of asthma on cardiorespiratory fitness requires clarification, with little consensus currently available in the literature (Berntsen et al., 2009, Pianosi \& Davis, 2004, Santuz et al., 1997, Vahlkvist \& Pedersen, 2009, Villa et al., 2011). These inter-study discrepancies may be attributable, at least in part, to the exercise testing methodologies used to determine cardiorespiratory fitness. Specifically, many 
children with asthma cite the fear of exercise-induced bronchoconstriction as a barrier to exercise (Chapter 4). As such, a testing method that is highly dependent on self-motivation and perceived ability, such as the 20-metre shuttle run test, a previously utilised estimate of cardiorespiratory fitness (Cairney et al., 2008, Mayorga-Vega et al., 2015), may be susceptible to producing poorer results in asthma participants that perceive themselves as less able.

Despite a fear of exercise-induced bronchoconstriction (Chapter 4), previous literature has suggested additional health benefits associated with exercise for adolescents with asthma such as reduced symptoms and severity and improved quality of life (Andrade et al., 2014), as shown in Chapter 5. Furthermore, adolescents with asthma have highlighted exercise as one of their favourite activities (Chapter 4), an encouraging finding for future exercise interventions seeking to address reports that few adolescents actively engage in exercise on a regular basis (Vahlkvist \& Pedersen, 2009). However, although significant improvements in fitness have been reported following exercise interventions in adolescents with asthma (Ahmaidi et al., 1993, Counil et al., 2003, van Veldhoven et al., 2001), these have been short in duration ( $<3$ months) and did not involve a follow-up. It is therefore unclear whether such improvements will be maintained, or indeed further enhanced, with an increased length of intervention or whether the changes will be maintained after intervention cessation.

Moderate-intensity, continuous exercise has predominately been utilised in previous exercise interventions in children with asthma (Basaran et al., 2006, Onur et al., 2011). However, adolescents with asthma report varied exercises, for example circuits or team games, as a preferred activity, with apprehension expressed towards long-distance running (Chapter 4). Such variation should avoid monotony which is associated with increased dropout rates (McNarry et al., 2015b). This highlights the potential utility of high-intensity interval training (HIIT) which has been suggested to be a time-efficient method of exercise that can elicit significant improvements in both cardiorespiratory fitness and body composition in youth (Costigan et al., 2015, Logan et al., 2014). Specifically, HIIT can be an effective means of improving body composition in young obese individuals (Lau et al., 2015), with significant improvements observed in both body mass index (BMI) and body fat percentage 
following recent 3-month interventions (Racil et al., 2016, Tjonna et al., 2009). Moreover, given past literature has indicated that asthma symptoms can also be triggered by continuous exercise (Sidiropoulou et al., 2007), intermittent exercise may not provoke asthma and might aid in improving adolescents' tolerance of exercise, increasing endurance (Del Giacco et al., 2015). Whilst increasing highintensity exercise tolerance, the intermittent nature of HIIT may also facilitate a decrease in end expiratory lung volume during the resting phase of the interval training (Beauchamp et al., 2010), reducing the chances of an asthma attack.

Therefore, the aim of the study was to investigate the effectiveness of an inclusive field-based six-month HIIT intervention on aerobic fitness, BMI, lung function and quality of life in adolescents with asthma. It was hypothesised that HIIT would lead to improvements in both cardiorespiratory fitness and quality of life and a reduction in BMI in adolescents, irrespective of asthma.

\subsection{Methods}

\subsubsection{Participants and study design}

In total, 616 adolescents (334 boys; Table 6.1), of which 155 had asthma (78 boys), agreed to participate in the study. In this randomised control trial, cluster randomisation was used to select one intervention school and four control schools in South Wales, matched for free school meal status (the X4A trial: eXercise for Asthma with Commando Joe's®). Two-hundred and twenty-one participants (116 boys) were recruited in the intervention school of the study, of which 47 suffered from asthma (24 boys). Asthma severity was assessed using the Global Initiative for Asthma guidelines (Global Initiative for Asthma, 2017) and classified as mild, moderate or severe according to the medication step required to achieve asthma control. For the purpose of analysis, moderate and severe asthma were grouped to power the statistics. Participants were excluded if they did not have stable asthma (n =4). Ethical approval was 


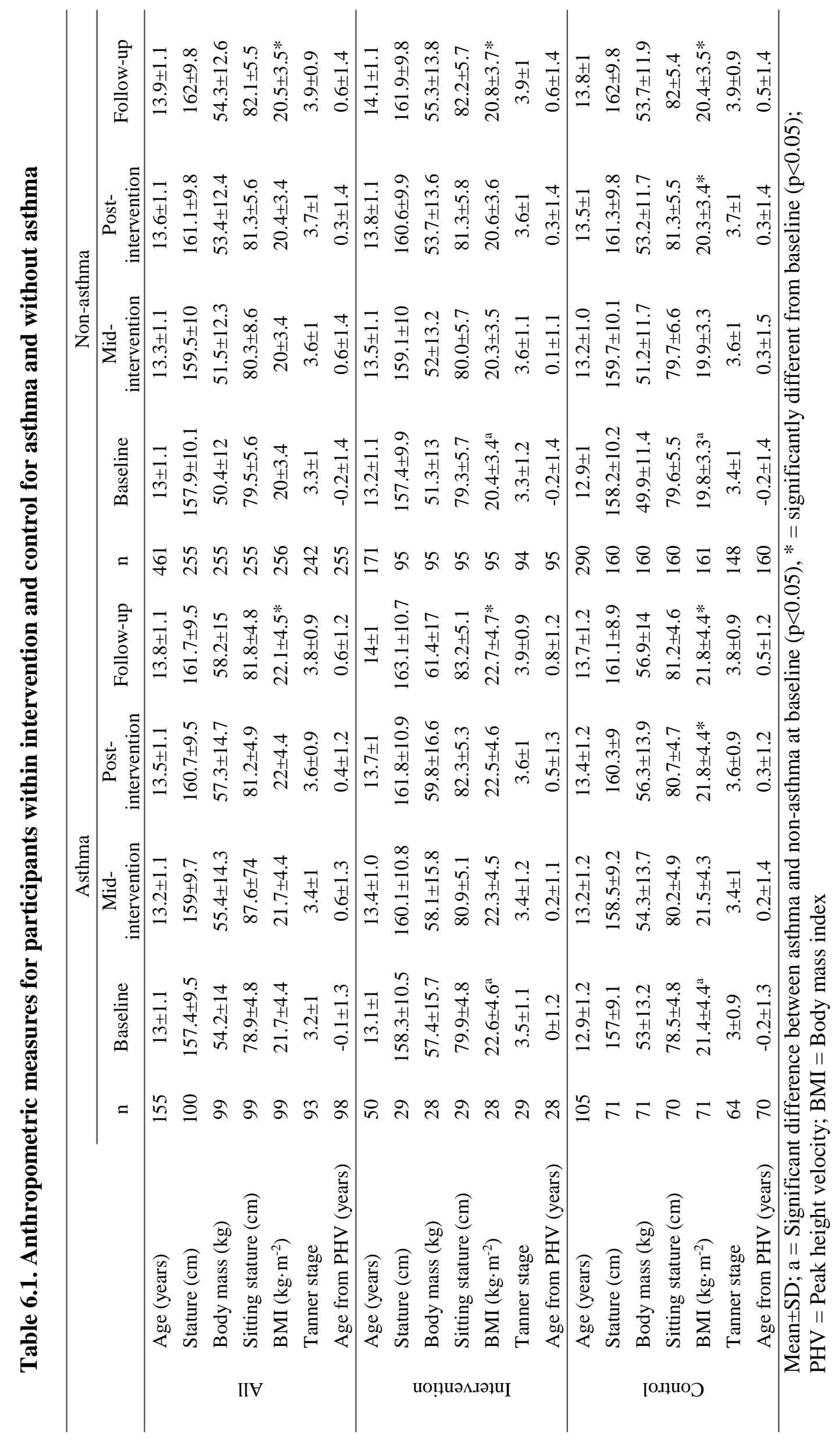


granted by the institutional research ethics committee (ref: 140515 and PG/2014/29). Parent/guardian and head teacher consent in addition to child assent were obtained prior to participation (see, appendix A).

\subsubsection{Intervention}

The intervention design was devised on previous research for intensity and duration (Wanrooij et al. 2014) and co-developed based on formative work to enable us to determine the elements and timing for the intervention (Chapter 4). The intervention consisted of a 6-month HIIT programme, delivered by Commando Joe's ${ }^{\circledR}$, involving $3 \times 30$-minute sessions per week. The sessions consisted of a combination of circuits and games-based activities (Table 6.2) lasting between 10 - 30 seconds, followed by an equal period of rest (Table 6.3). Throughout each exercise bout, participants were asked to exercise maximally with exercise activities designed to elicit a heart rate (HR) of $>90 \%$ of heart rate maximum (HRmax, Hood et al., 2011). Maximal HR was predicted according to Tanaka, Monaham and Seals (2001), which has been validated for use in children and adolescents (Mahon et al., 2010). During each session participants' HR was continuously monitored (Activio AB, Stockholm, SWE). Those in the control group engaged in their usual day-to-day activities.

\subsubsection{Procedures}

Measurements were taken from both intervention and control groups at four-time points (baseline, mid-intervention, post-intervention and at 3-month follow-up), irrespective of condition.

\subsubsection{Anthropometrics}

Stature and body mass were measured according to the techniques outlined by International Society for the Advancement of Kinanthropometry (Stewart et al., 2011). Stature, sitting stature and waist circumference were measured to the nearest $0.1 \mathrm{~cm}$ (Seca213, Hamburg, Germany) and body mass to the nearest $0.1 \mathrm{~kg}$ (Seca876, Hamburg, Germany). Body mass index was subsequently calculated and grouped using age and sex specific child percentiles (Barlow, 2007). Maturity offset 
was calculated according to Mirwald et al. (2002), lower limb length was calculated as the difference between stature and sitting stature

\subsubsection{Lung Function}

Forced Expiratory Volume in 1 second $\left(\mathrm{FEV}_{1}\right)$, Forced Vital Capacity (FVC), $\mathrm{FEV}_{1} / \mathrm{FVC}$ ratio, Peak Expiratory Flow (PEF), and Forced Expiratory Flow between $25-75 \%$ of vital capacity $\left(\mathrm{FEF}_{25-75}\right)$ was measured using a portable dry spirometer (Vitalograph, Buckingham, UK). The best of three measurements were taken according to American Thoracic Society guidelines (1995) and to the standardised protocol (Miller et al., 2005) and expressed as a percentage of the age-sex-stature predicted value (Rosenthal et al., 1993).

\subsubsection{Fractional Exhaled Nitric Oxide}

Fractional exhaled nitric oxide (FeNO) was measured prior to spirometric testing. The FeNO test was performed in accordance with the American Thoracic Society guidelines (Dweik et al., 2011). Participants were asked to completely exhale and then inhale to total lung capacity through the device (NIOX MINO, Aerocrine AB, Solna, Sweden), before immediately exhaling for 10 seconds at $50 \pm 5 \mathrm{ml} \cdot \mathrm{sec}^{-1}$. Visual and audio cues were provided by the computer software throughout. One test was completed at all time-points except follow-up. The final three seconds of exhalation were evaluated.

\subsubsection{Asthma control}

Asthma control was assessed using the Asthma Control Questionnaire (ACQ, Juniper et al., 2010) which consists of 7-items focusing on reliever inhaler use, symptoms and $\mathrm{FEV}_{1}$ score. Items of the ACQ were scored from 0 to 6, with ACQ scores of $\leq 0.75$ or $\geq 1.5$ indicating well-controlled and poorly-controlled asthma, respectively. Internal consistency, measured using Cronbach's alpha coefficients (Cronbach, 1951), for the ACQ were deemed acceptable $(\alpha=0.73-0.82)$. 
Table 6.2 Detailed examples of exercises

Activity $\quad$ Example exercises

\begin{tabular}{|c|c|}
\hline $\begin{array}{l}\text { Accumulator } \\
\text { circuits }\end{array}$ & $\begin{array}{l}\text { Participants had to run a short distance and back and complete one repetition } \\
\text { of the first exercise. On the second interval, they had to run out and } \\
\text { complete one repetition of the first exercise and two repetitions of the } \\
\text { second. The pattern continued adding one rep each time. Participants would } \\
\text { continue from where they left off at each rest until the exercises were all } \\
\text { "accumulated". Example: }\end{array}$ \\
\hline & $\begin{array}{ll}\text { - } & \text { Jumping jacks } \\
\text { - } & \text { Worm push ups } \\
\text { - } & \text { Squats } \\
\text { - } & \text { Burpees }\end{array}$ \\
\hline
\end{tabular}

Static exercises Participants stood in a space where they conducted exercises. Example:

- Burpees

- Jumping jacks

- Squats

- $\quad$ Press-ups

Obstacle course Created using resources such as nets, benches, cones and hurdles. Example:

- Agility

- Crawling

- Jumping

- Rolling

Speed, agility and Activities included fast paced, low skill agility exercises such as: quickness

- Zig-zag sprints

- Slalom sprints

- Hurdles

- Shuttles

- $\quad$ Fast feet ladder drills

Linear sprints Participants moved between each side of the hall. Movement was varied i.e. forward, backwards and sideways. Variations to the sprints included:

- Bear crawls

- Gorilla walks

- Crab walks 
Table 6.3. Example exercise sessions from the high-intensity interval training intervention

\begin{tabular}{lc}
\hline & Exercise/Rest Time (s) \\
\hline Week 1-3 & $10 / 10$ \\
& $10 / 10$ \\
& $10 / 10$ \\
\hline Week 4-6 & $15 / 15$ \\
& $15 / 15$ \\
& $15 / 15$ \\
\hline & $30 / 30$ \\
Week 7+ & $30 / 30$ \\
& $30 / 30$ \\
\hline
\end{tabular}

\subsubsection{Asthma-related quality of life}

Symptoms, activity limitation and emotional and environmental effects of asthma were assessed using the Paediatric Asthma Quality of Life Questionnaire (PAQLQ, Juniper et al., 1996). The PAQLQ consists of 23 questions (scored on a Likert scale from 1 to 7), with a higher score indicative of a better asthma status. Internal reliability for the PAQLQ was deemed excellent ( $\alpha=0.96-0.97)$.

\subsubsection{Quality of life}

The Pediatric Quality of Life Inventory (PedsQL) Teenager Report (Version 4.0, Varni et al., 1999) was used to compare the perceived quality of life between those participants with and without asthma. A widely validated measure in adolescents (Varni et al., 2006, Varni et al., 2003, Varni et al., 2001), the 23-item PedsQL consists of questions on the participants' physical, emotional, social and school functioning quality, with higher scores indicating a better quality of life. Internal reliability for the PedsQL was deemed excellent $(\alpha=0.89-0.90)$. 


\subsubsection{Cardiorespiratory fitness}

20-metre shuttle run

Cardiorespiratory fitness was estimated using the 20-metre progressive shuttle run test, a previously validated field measure in children (Mayorga-Vega et al., 2015). The number of shuttles completed before voluntary exhaustion were recorded.

\section{Peak $\dot{V} \mathrm{O}_{2}$}

Sixty-nine adolescents ( 39 boys) inclusive of 36 with asthma (21 boys) were selected using stratified randomisation to complete incremental ramp tests. The groups were stratified for age, sex and condition to provide a representative sample of the wider population. Participants performed an incremental ramp exercise test to volitional exhaustion on an electromagnetically-braked cycle ergometer (Ergoselect 200, Ergoline $\mathrm{GmbH}$, Lindenstrasse, Germany), with individually-adjusted seat and handlebar height. The ramp protocol consisted of 3 minutes of "unloaded" pedalling $(0 \mathrm{~W})$ followed by an increase in work rate of $12-24 \mathrm{~W} \cdot \mathrm{min}^{-1}$ dependant on the age and height of the participant. Participants were asked to maintain a constant cadence $(75 \pm 5$ revolutions per minute) until voluntary exhaustion. Breath-by-breath pulmonary ventilation (VE) and gas exchange $\left(\dot{V} \mathrm{O}_{2}\right.$ and $\left.\dot{V} C \mathrm{O}_{2}\right)$ were recorded throughout (Jaeger Oxycon Mobile, Jaeger, Hoechberg, Germany).

\subsubsection{Data analysis}

Peak $\dot{V} \mathrm{O}_{2}$ was taken as the highest 10 -second mean attained prior to the end of the test. The gas exchange threshold (GET) was determined using the V-slope method (Beaver et al., 1986). The GET was also expressed relative to peak $\dot{V} \mathrm{O}_{2}$ (GET\% $\dot{V} \mathrm{O}_{2}$ ). Analysis of covariance (ANCOVA) was used to determine the allometric relationship between peak $\dot{V} \mathrm{O}_{2}$ and body mass to account for body size using logtransformed data. Common allometric exponents were confirmed for the data and power function ratios $\left(\mathrm{Y} / \mathrm{X}^{\mathrm{b}}\right)$ were computed. Breath-by-breath data were then averaged into 10-second time bins and the Mean Response Time (MRT) and gain $\left(\Delta \dot{V} \mathrm{O}_{2} / \Delta \mathrm{WR}\right)$ calculated according to the methods reported by Barstow et al. (2000b). Specifically, the gain was determined by linear regression over three 
segments: $\mathrm{S}_{1}$, from 1-minute into the ramp to GET; $\mathrm{S}_{2}$, from GET to peak $\dot{V} \mathrm{O}_{2}$; and $\mathrm{S}_{\mathrm{T}}$, over the total range of $\mathrm{S}_{1}+\mathrm{S}_{2}$. Baseline $\dot{V} \mathrm{O}_{2}$ was taken as the mean of the first 45 seconds of the last minute prior to the increase in work rate. The MRT was calculated as the point of intersection between the baseline $\dot{V} \mathrm{O}_{2}$ and a backwards linear extrapolation of the $\dot{V} \mathrm{O}_{2}$ by time slope from the onset of the ramp protocol (Glantz, 1990). The MRT was also determined using two segments, $\mathrm{S}_{1}\left(\mathrm{MRT}_{1}\right)$ and $\mathrm{S}_{\mathrm{T}}$ (MRTT, Whipp et al., 1981).

\subsubsection{Statistical analysis}

Shapiro-Wilk tests were used to assess normality. Following identification of normal distribution, the influence of asthma and the intervention, and their interaction, was assessed using a mixed-model ANOVA (groups - asthma intervention, non-asthma intervention, asthma control, non-asthma control). Tukey's post-hoc analyses were conducted to ascertain where differences in time were found. If significant differences were found, mixed-design ANCOVA tests were run to adjust for baseline maturity. Asthma-specific measures were analysed using repeated measures ANOVAs. All analyses were conducted using an intention-to-treat approach, thereby including all participants with measures at any time-point; data were subsequently analysed using sensitivity analysis on participants who participated in the majority of the intervention sessions $(>70 \%)$. Eta-squared $\left(\eta_{\mathrm{p}}{ }^{2}\right)$ effect sizes were determined from baseline to follow-up. All statistical analyses were conducted using SPSS v22 (IBM Corp, Armonk, NY). All data are presented as mean \pm standard deviation (SD) with statistical significance accepted as $\mathrm{P}<0.05$.

\subsection{Results}

The participants with asthma in the intervention group consisted of $87 \%$ with mild persistent and $13 \%$ with moderate or severe asthma, whilst the participants with asthma in the control group consisted of $77 \%$ and $23 \%$, respectively. This prevalence was similar in both the intention-to-treat and sensitivity analyses. Where no differences between intention-to-treat and sensitivity analysis were found, results 
refer to the former. Furthermore, no differences were observed when co-varying for maturity offset or Tanner stages and are therefore not reported below.

\subsubsection{Lung function}

A lower $\mathrm{FEV}_{1} \%$ and $\mathrm{FEF}_{25-75}$ were found in participants with asthma indicating more airway obstruction and more marked small airways obstruction, respectively (Table 6.4). Those with asthma did not have an obstructed $\mathrm{FEV}_{1} / \mathrm{FVC}$ ratio. Mixed methods ANOVAs revealed no differences between intervention and control, asthma and non-asthma for lung function $(\mathrm{P}>0.05)$ according to group or time or a time by group interaction. There was, however, a trend for FeNO to reduce in the intervention asthma group.

\subsubsection{Asthma control and quality of life}

The intervention had no effect on asthma control or asthma-related quality of life. The Minimal Important Difference (MID) for both the ACQ and PAQLQ was a change in score of 0.5. Both intervention and control asthma participants demonstrated similar results, with 33 and $35 \%$ and 19 and $16 \%$ for ACQ and PAQLQ, respectively, scoring above the MID. Similarly, for the PedsQL, there were no differences either by time or group, or interactions between time and group (Table $6.5)$.

\subsubsection{Body Mass Index}

Body Mass Index was found to be significantly higher in participants with asthma at baseline in comparison to their peers. There was a significant effect of time on BMI $\left(F(2.23,782)=15.4, \mathrm{P}<0.05 \eta_{\mathrm{p}}{ }^{2}=0.04\right)$, and a significant difference between groups $\left(F(3,351)=5.29, \mathrm{P}<0.05 \eta_{\mathrm{p}}^{2}=0.04\right)$. However, there was no interaction between time and group $\left(F(6.68,782)=1.16, \mathrm{P}=0.33 \eta_{\mathrm{p}}{ }^{2}=0.01\right)$. Post-hoc analyses revealed both asthma and non-asthma control groups, experienced significant increases $(\mathrm{P}<0.05)$ in BMI between baseline and post-intervention $(21.4$ \pm 4.4 to $21.8 \pm 4.4 \mathrm{~kg} \cdot \mathrm{m}^{-2}$ and $19.8 \pm 3.3$ to $20.3 \pm 3.4 \mathrm{~kg} \cdot \mathrm{m}^{-2}$, asthma and nonasthma, respectively). No increases across the intervention were found in either of 


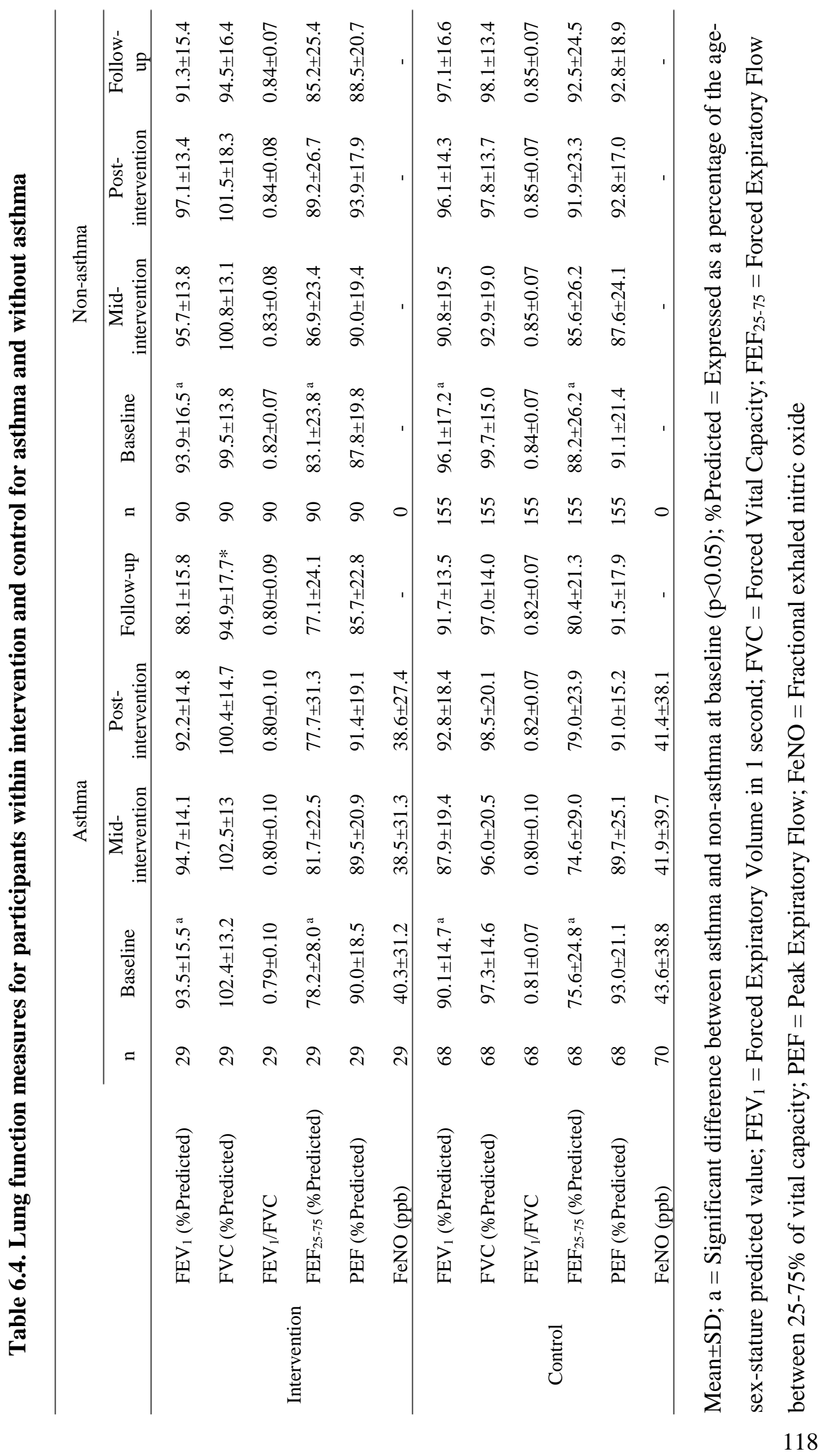




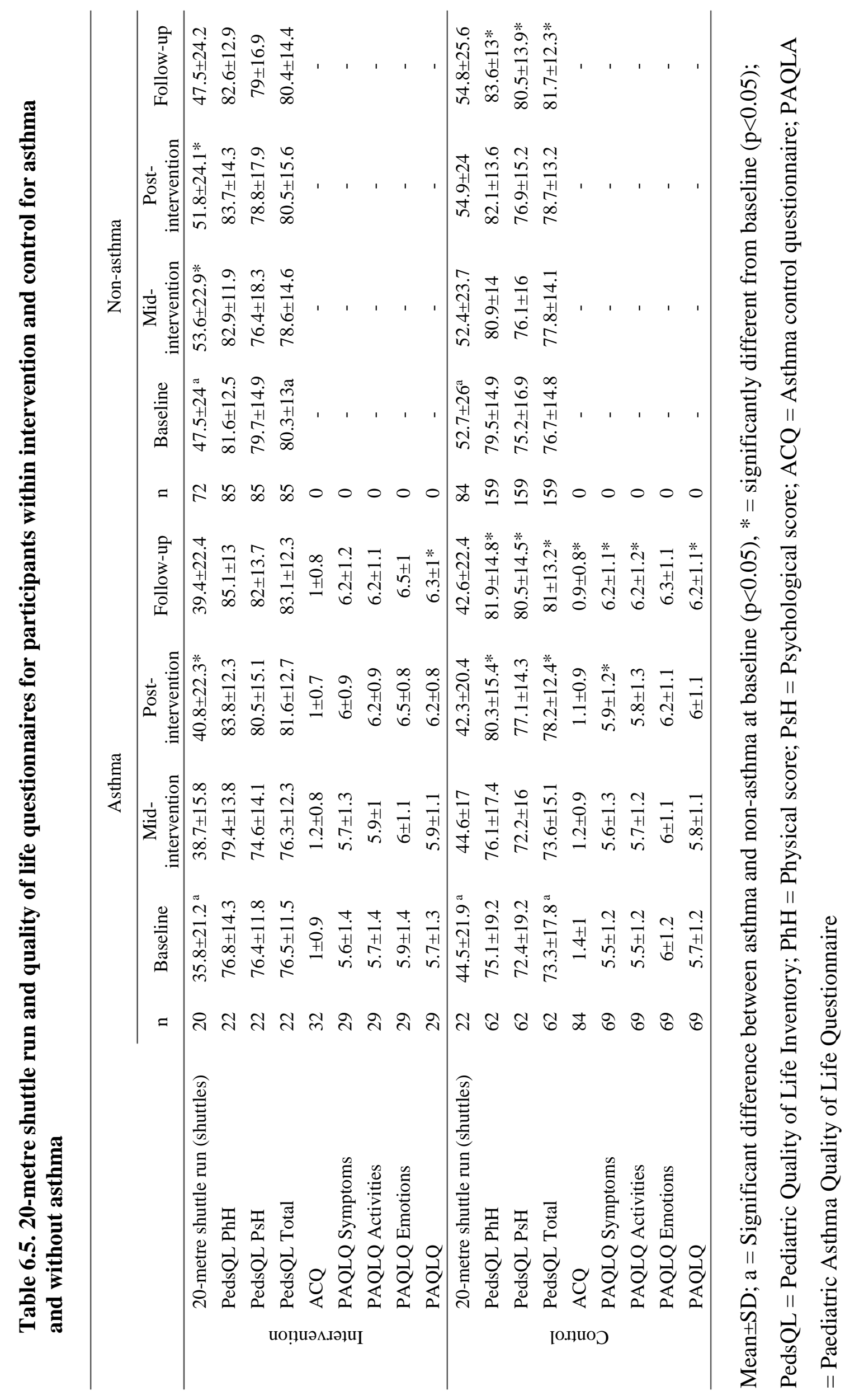


the asthma or non-asthma intervention groups. Body Mass Index was significantly higher than baseline in all groups at follow-up.

\subsubsection{0-metre shuttle run}

No significant effects were found for group, time or group by time interaction for the 20-metre shuttle run. However, when applying sensitivity analyses, there was a significant effect of time $\left(F(3,386)=5.44, \mathrm{P}<0.05 \eta_{\mathrm{p}}{ }^{2}=0.04\right)$ and a significant interaction of group by time $\left(F(9,386)=3.23, \mathrm{P}<0.05 \eta_{\mathrm{p}}{ }^{2}=0.06\right)$. Post-hoc analyses revealed a significant increase in the number of shuttles completed in both asthma and non-asthma intervention groups with time, which returned to baseline at follow-up.

\subsubsection{Incremental ramp test}

A significant effect of time and interaction between time and the group was observed, with no significant effect of group on peak $\dot{V} \mathrm{O}_{2}$. When scaled for body size, these differences were maintained with time $\left(F(3,138)=8.47, \mathrm{P}<0.05 \eta_{\mathrm{p}}{ }^{2}=\right.$ $0.16)$, group by time $\left(F(9,138)=2.70, \mathrm{P}<0.05 \eta_{\mathrm{p}}^{2}=0.15\right)$, and group $(F(3,46)=$ 1.55, $\left.\mathrm{P}=0.22 \eta_{\mathrm{p}}{ }^{2}=0.09\right)$. Post-hoc analyses revealed significant increases in peak $\dot{V} \mathrm{O}_{2}$ in both asthma and non-asthma intervention groups, with follow-up results showing a return to baseline levels. No differences were observed in either of the asthma or non-asthma control groups across the intervention for peak or scaled peak $\dot{V} \mathrm{O}_{2}$ (Table 6.5).

There were no differences in GET between groups, however, there was a significant increase over time in all groups $\left(F(2.23,138)=41.56, \mathrm{P}<0.05 \eta_{\mathrm{p}}{ }^{2}=0.48\right)$. There was no significant between group differences for GET as a percentage of peak $\dot{V} \mathrm{O}_{2}$. Post-hoc analyses showed significant increases at post-intervention for the nonasthma intervention and both asthma and non-asthma control groups, however, inclusive of the asthma intervention group, all groups significantly increased from baseline to follow-up. Sensitivity analysis also showed that there were no significant increases throughout the intervention in GET\% $\dot{V} \mathrm{O}_{2}$ for participants in the nonasthma intervention group. There were no significant differences to either section of 


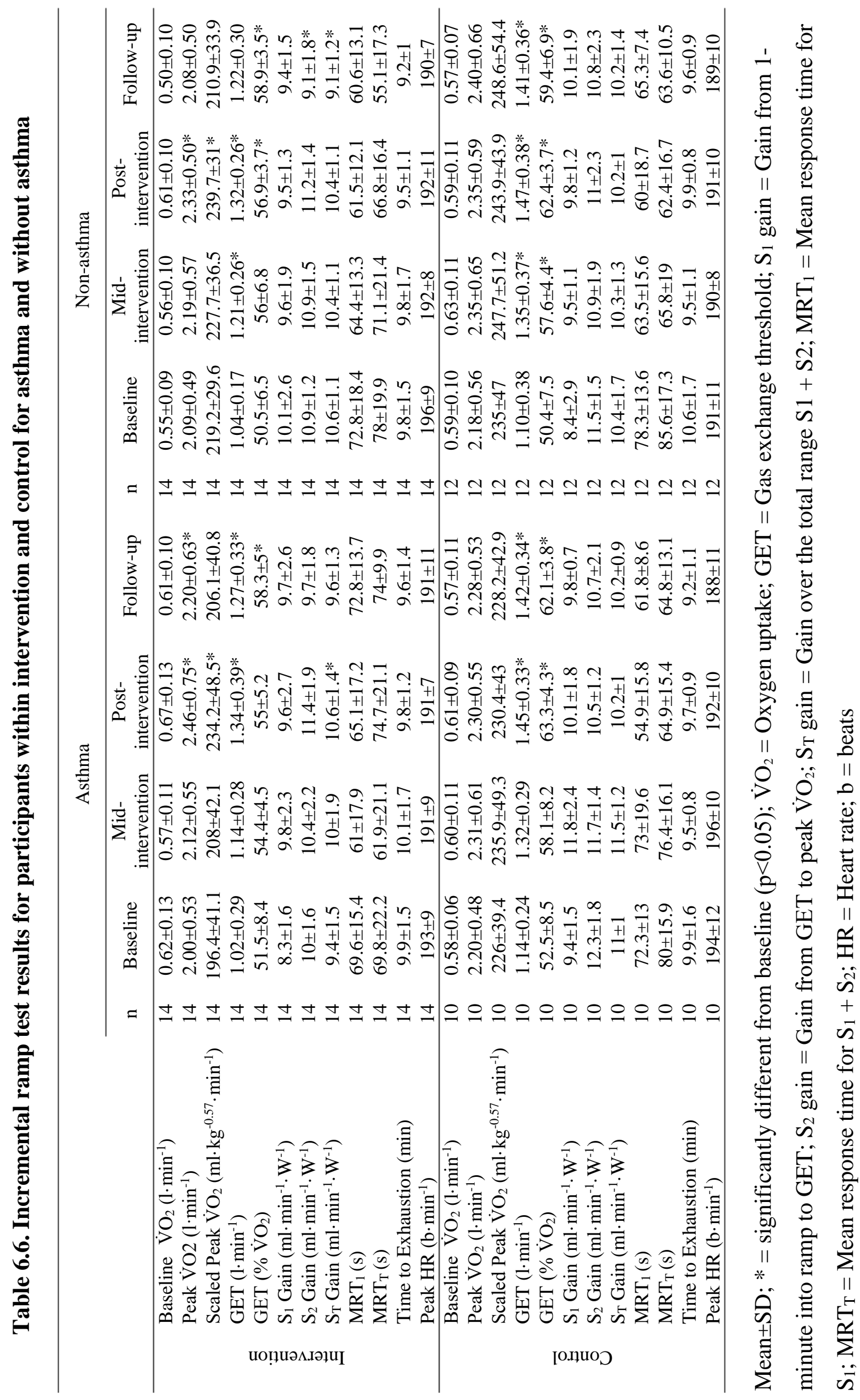


the MRT according to time, group or time by group interaction across all timepoints. The gain, however, was found to significantly increase in the intervention asthma group for both $S_{2}$ and $S_{T}$, with no significant differences observed in any of the other groups.

\subsubsection{Intervention intensity}

Throughout the intervention sessions, exclusive of warm-up and cool-down, participants' mean HR (155 \pm 18 beats per minute $\left.(\mathrm{bpm}), 78 \pm 9 \% \mathrm{HR}_{\max }\right)$ and mean $\mathrm{HR}_{\max }\left(188 \pm 18 \mathrm{bpm}, 95 \pm 6 \% \mathrm{HR}_{\max }\right)$ were calculated for each session. During the main body of the session, inclusive of both the exercise and rest intervals, HR exceeded the threshold of $>90 \% \mathrm{HR}_{\max } 24 \%$ of the total time.

\subsubsection{Correlations}

All measures were positively correlated with themselves between baseline and postintervention, with the exception of the MRT and gain. A weak negative correlation was observed between BMI and fitness $(r=-0.34, \mathrm{P}<0.05)$, quality of life $(r=-$ $0.11, \mathrm{P}<0.05)$ and lung function $(\mathrm{r}=-0.21, \mathrm{P}<0.05)$ at baseline, but only fitness $(\mathrm{r}$ $=-0.33, \mathrm{P}<0.05)$ at post-intervention. Fitness was also weakly correlated with quality of life $(\mathrm{r}=0.26, \mathrm{P}<0.05)$ and lung function $(\mathrm{r}=0.34, \mathrm{P}<0.05)$ at all time-

points. However, scaled peak $\dot{V} \mathrm{O}_{2}$ was not associated with quality of life or lung function $(\mathrm{P}>0.05)$.

\subsection{Discussion}

The main finding of this study was that HIIT elicited significant improvements in cardiorespiratory fitness and maintained BMI in adolescents, irrespective of condition. However, HIIT did not elicit significant improvements in lung function, asthma control or quality of life. Taken together, these findings suggest similar physiological responses to HIIT for asthma adolescents in comparison to their healthy peers. Such findings have significant implications and should be taken into consideration in the design of future interventions as previously adolescents with asthma have been treated differently through exclusion and the perception they are 
unable to participate and "keep-up" during similar activities to their peers (Trollvik et al., 2011, van den Bemt et al., 2010).

Congruent with previous research (Taylor et al., 2015), the present study found that HIIT was associated with increased cardiorespiratory fitness. Specifically, 20-metre shuttle run scores significantly improved, irrespective of condition, with no significant changes noted for the controls. Furthermore, sub-sample analyses for both absolute and body size scaled peak $\dot{V} \mathrm{O}_{2}$ further supported changes in cardiorespiratory fitness. Interestingly, the asthma intervention group increased their scaled $\dot{V} \mathrm{O}_{2}$ to a greater extent than their non-asthma peers (19 vs. 10\%), though such findings may have been due to the lower, albeit non-significant, baseline scores for those with asthma. Indeed, baseline fitness has been reported to influence the magnitude of change elicited by an intervention in youth (Eliakim et al., 1996, Mandigout et al., 2001, Tolfrey et al., 1998). Although similar improvements in peak $\dot{V} \mathrm{O}_{2}$ following moderate-intensity exercise have been noted in those with asthma (5$10 \mathrm{ml} \cdot \mathrm{min}^{-1} \cdot \mathrm{kg}^{-1}$ ) over a shorter time-frame (Ahmaidi et al., 1993, Counil et al., 2003, Varray et al., 1995, Varray et al., 1991), the suitability of continuous exercise in those with asthma is questionable. Indeed, research has suggested that prolonged monotonous exercise is not enjoyable (McNarry et al., 2015b) and has been shown to be a barrier to those with asthma (Chapter 4). Furthermore, it has been suggested that asthma symptoms may be triggered by continuous exercise (Sidiropoulou et al., 2007), highlighting the potential utility of HIIT.

Although peak aerobic fitness significantly increased, in line with Baquet et al. (2001), GET appeared to be unaffected by the HIIT intervention for both those with and without asthma, suggesting that training above the GET for short intermittent periods may not elicit improvements. The GET\% $\dot{V} \mathrm{O}_{2}$ were similar at baseline to previous studies (McNarry et al., 2015a), however, contrary to HIIT in normal weight children (McNarry et al., 2015b), GET significantly increased in both intervention and control groups within the present study. This suggest that increases in GET are possibly due to the maturational changes in both those in the intervention and control during a period of rapid growth that may have occurred during that time (Breese et al., 2010), although a larger study is required to confirm this suggestion. 
In addition to the GET, irrespective of condition, HIIT did not significantly improve the MRT. These findings are somewhat surprising considering the nature of HIIT training, involving repeated transitions to vigorous intensity exercise from rest. Intriguingly, the MRT in the present study was longer than previously reported in healthy children (Barstow et al., 2000a, McNarry et al., 2011a). Inter-study comparisons are limited, however, as the ramp rate of the incremental test has been found to profoundly affect the MRT (Wilcox et al., 2016). Interestingly, there were no differences in MRT between those with and without asthma, suggesting asthma does not impede the response to exercise.

The increase in gain observed over the intervention in participants with asthma is suggestive of a positive adaptation in the delivery and utilisation of oxygen by the muscles during exercise (Neder et al., 2001). Of note, although no differences in gain were observed at baseline, $S_{2}$ and $S_{T}$ gain increased post-intervention for participants with asthma to similar levels to those reported elsewhere in healthy adolescents (Fielding et al., 2015). This increase in gain may indicate that HIIT elicits different adaptations in those with and without asthma, although this may also be a function of the lower baseline levels in those with asthma allowing greater capacity for improvement. The lower levels of aerobic efficiency in participants with asthma may be related to a decreased lung function and may be a contributory mechanism to the onset of early fatigue and the perception that people with asthma are not as fit as their peers, although it is worth noting they were not correlated in the current study. Indeed, Fielding et al. (2015), found a reduced gain in Cystic Fibrosis patients and attributed this to, at least in part, explain the reduced exercise intolerance in Cystic Fibrosis when compared to their healthy peers. Importantly, the current study demonstrates that the gain of participants with asthma can be improved with a HIIT programme.

Cardiorespiratory fitness was found to have a weak but significant correlation with quality of life in those with asthma at baseline. Therefore, improving fitness could possibly aid in the self-management of asthma through a potential reduction of symptoms and increased ability to participate in exercise with peers over a prolonged period. However, contrary to previous exercise interventions (Basaran et al., 2006, Fanelli et al., 2007), quality of life did not change over time, irrespective of 
treatment group or asthma status, perhaps due to the overall weak association with cardiorespiratory fitness. Furthermore, there was no change over time for perceived asthma-related quality of life, symptoms or asthma control. It could be postulated that the lack of improvement in asthma-related quality of life may be due to the mild severity of asthma or participants having high baseline values (Fanelli et al., 2007, Moreira et al., 2008, Wanrooij et al., 2014), thereby decreasing the likelihood of an effect, or indeed need for an effect.

Whilst the present study is consistent with the majority of the literature which similarly found that exercise did not affect lung function (Wanrooij et al., 2014), it is pertinent to note that two studies reported a significant increase in $\mathrm{FEV}_{1} \%$ (8-20\%), both of which implemented intermittent training (Latorre-Roman et al., 2014, Sidiropoulou et al., 2007). This discrepancy may, in part, be related to the severity of asthma, with the majority of participants in this study having mild asthma, or to the intervention duration; although longer than many previous studies (Moreira et al., 2008, Wicher et al., 2010), 6-months may have been insufficient to elicit significant adaptations in lung function. The inflammatory biomarker (FeNO) was found to be unchanged over the intervention with similar findings to that of a previous exercise training intervention (Moreira et al., 2008). The variability of FeNO was very large $(\mathrm{CV}=103 \%)$ and therefore no real conclusions can be drawn. Furthermore, as $70 \%$ of the participants were on inhaled corticosteroids, this may have masked any potential exercise effects. Interestingly, although weight reduction has previously been shown to improve lung function in the obese/overweight (Latorre-Roman et al., 2014), within the present study, the increase in BMI in the control group did not manifest in any reduction in lung function. Consequently, future studies incorporating a longer duration intervention should endeavour to clarify effects on BMI and lung function. The current increase in control group BMI is in accord with previous literature showing a linear increase in BMI with age (de Souza et al., 2015), therefore, the intervention may be able to preclude the typical rise in BMI with age in both those with and without asthma. The maintenance rather than a reduction of BMI in the intervention group is perhaps a healthier finding considering the "normal" BMI of the participants. However, compensatory decreases in physical 
activity, often observed outside of interventions (Ridgers et al., 2018a), may have precluded a reduction in BMI.

Key strengths of the present study were the more sensitive measures of aerobic fitness (GET, MRT and gain) which have not previously been assessed across multiple time-points in adolescents with asthma. Nonetheless, several limitations should be acknowledged. As with any exercise intervention, there may have been a self-selection bias with voluntary participant recruitment. In addition, participants in the control schools may have increased their physical activity levels, or those in the intervention school may have reduced their time within physical activity, potentially altering the conclusions drawn from this study. Indeed, this highlights the importance of physical activity monitoring within exercise intervention studies, a measure that we tried to incorporate, however, unfortunately the adherence to this measure was poor and it is therefore not reported.

Furthermore, although the HIIT intervention was designed using formative research (Chapter 4) in order ensure that it was enjoyable and cater to everyone's needs, participants who signed up to the intervention either committed fully, attending a large proportion of the 6-months sessions, or had minimal attendance over the intervention. The attendance resulted in the use of an intention-to-treat strategy and sensitivity analysis, which may have resulted in a loss of power possibly reducing the significance of the findings. Such lack of attendance questions the utility of the intervention; however, it could be postulated that the timing of the exercise sessions reduced participation and that if more optimal timings were possible, a stronger adherence could have been achieved. Finally, as participants with asthma were not restricted from using their medication, this could account for the lack of difference in cardiorespiratory fitness between adolescents with and without asthma.

\subsection{Conclusion}

In conclusion, a HIIT intervention may be an effective tool to increase peak aerobic fitness and prevent increases in BMI in adolescents, irrespective of asthma. Of importance, adolescents with asthma elicited similar physiological adaptations in 
comparison to their healthy peers, thereby demonstrating that asthma does not influence aerobic fitness or trainability in adolescents. Furthermore, the lack of exercise-induced asthma attacks suggests that HIIT is safe for, and well-tolerated by, adolescents with predominately mild asthma when on prescribed medication. 


\section{Thesis study map}

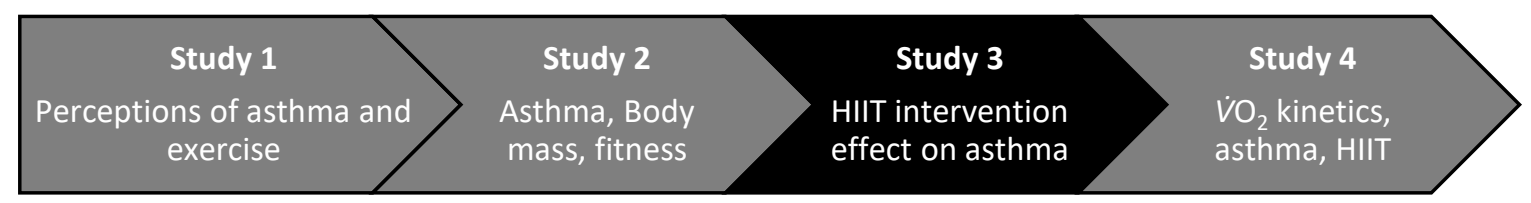

\section{Chapter 6}

Aims:

- To investigate the effectiveness of an inclusive field-based six-month HIIT intervention on aerobic fitness, BMI, lung function and quality of life in adolescents with asthma

Key findings for future chapters

- HIIT is safe for adolescents with predominantly mild asthma

- HIIT was unable to elicit significant changes in lung function or quality of life

- Irrespective of asthma, HIIT may aid in maintaining BMI avoiding increases associated with maturation

- Irrespective of asthma, HIIT may significantly improve peak cardiorespiratory fitness but might not have an impact on submaximal fitness 


\section{CHAPTER 7}

\section{EFFECT OF HIGH-INTENSITY INTERVAL TRAINING ON PULMONARY OXYGEN UPTAKE KINETICS IN ADOLESCENTS WITH ASTHMA}




\section{Chapter 7: Effect of high-intensity interval training on pulmonary oxygen}

uptake kinetics in adolescents with asthma

This Study has been disseminated as follows:

Oral presentation: Winn CON, Mackintosh KA, Eddolls WTB, Stratton G, Wilson AM, McNarry MA, Davies GA (2017). The influence of a six-month high-intensity interval training intervention on the pulmonary oxygen uptake kinetics in adolescents with and without asthma. European College of Sport Science annual congress; Essen, Germany

\subsection{Introduction}

Although peak $\dot{V} \mathrm{O}_{2}$ is widely considered the gold standard measure of aerobic fitness (Carey \& Richardson, 2003), its relevance and applicability to daily life is questionable (Jones \& Poole, 2005). As such, the interpretation of previous studies investigating the influence of asthma on cardiorespiratory fitness is limited by their reliance on peak oxygen uptake $\left(\dot{V} \mathrm{O}_{2}\right)$. Indeed, sub-maximal fitness accrued through sporadic daily activity is arguably better assessed by $\dot{V} \mathrm{O}_{2}$ kinetics which reflect the dynamic $\dot{V} \mathrm{O}_{2}$ response to an instantaneous change in the metabolic demand (Grassi et al., 1996, Krustrup et al., 2009). The $\dot{V} \mathrm{O}_{2}$ kinetic response has been reported to be highly sensitive to both exercise training and disease in adults, although considerably less is known regarding the influence of such stimuli in youth populations. In healthy adults, training is associated with a faster $\dot{V} \mathrm{O}_{2}$ phase II time constant (Murias et al., 2010, Raleigh et al., 2016), however, studies in children have shown contradictory results. Specifically, a faster $\dot{V} \mathrm{O}_{2}$ kinetic time constant was reported in trained compared to untrained children (Winlove et al., 2010) with no differences found in two other studies (Cleuziou et al., 2002, Obert et al., 2000). Adolescent studies have, however, found training to be associated with a faster $\dot{V} \mathrm{O}_{2}$ kinetic time constant (Marwood et al., 2010, McNarry et al., 2011c, Unnithan et al., 2015). A faster $\dot{V} \mathrm{O}_{2}$ kinetic time constant was also observed following a 6-week High-Intensity Interval Training (HIIT) intervention in obese children (McNarry et al., 2015b), though it is noteworthy that no influence of HIIT was found in the normal weight children. 
The determinants of the dynamic $\dot{V} \mathrm{O}_{2}$ response may be displaced by disease (Poole \& Jones, 2012). Therefore, the derangements in the airways associated with asthma may hinder the response to exercise with respect to $\mathrm{O}_{2}$ delivery and utilisation. Indeed, recent respiratory disease studies found impaired $\dot{V} \mathrm{O}_{2}$ kinetics in those with Emphysema and Idiopathic Pulmonary Fibrosis (McNarry et al., 2017), Cystic Fibrosis (Saynor et al., 2016) and Chronic Obstructive Pulmonary Disease (Nery et al., 1982, Puente-Maestu et al., 2000) compared with age-matched healthy counterparts. This may be attributable to an impaired $\mathrm{O}_{2}$ delivery consequent to mismatched ventilation and gas exchange in the lung, causing a low arterial $\mathrm{O}_{2}$ content (Jones \& Poole, 2005).

Despite widespread interest in HIIT, little is known about the effect of HIIT in children or adolescents, and particularly its interaction with asthma. Therefore, the aim of this study was to investigate the influence of asthma and HIIT, and their interaction, on the dynamic $\dot{V} \mathrm{O}_{2}$ response in adolescents. It was hypothesised that the participants with asthma would have slower $\dot{V} \mathrm{O}_{2}$ kinetics than their healthy peers and that the HIIT intervention would increase the peak $\dot{V} \mathrm{O}_{2}$ and speed $\dot{V} \mathrm{O}_{2}$ kinetics in both groups.

\subsection{Methods}

\subsubsection{Participants and study design}

Sixty-nine adolescents (39 boys, $13.6 \pm 0.9$ years; 36 with asthma, 21 boys) were selected using stratified randomisation from 618 participants (Table 7.1) across two schools (one intervention and one control). These groups were stratified for age, sex and condition (asthma/non-asthma) to provide a representative sample of the wider population. Participating schools were randomly selected from fifteen schools initially invited to take part in the randomised control trial (the $\mathrm{X} 4 \mathrm{~A}$ trial: eXercise for Asthma with Commando Joe's). Asthma severity was assessed using the Global Initiative for Asthma guidelines (Global Initiative for Asthma, 2017) and classified as mild, moderate or severe according to the medication step required to achieve asthma control. For the purpose of analysis, moderate and severe asthma were 
grouped to power the statistics. Participants were excluded if they did not have stable asthma $(\mathrm{n}=1)$. Ethical approval was granted by the institutional research ethics committee (ref: 140515 and PG/2014/29). Parent/guardian and head teacher consent as well as child assent were obtained prior to participation (see appendix A).

\subsubsection{Intervention}

The intervention design was devised on previous research for intensity and duration (Wanrooij et al. 2014) and co-developed based on formative work to enable us to determine the elements and timing for the intervention (Chapter 4). Participants within the intervention group were required to attend a HIIT intervention, three days per week, for six-months, in accord with recommendations from recent systematic reviews (Eddolls et al., 2017, Wanrooij et al., 2014). The 30-minute intervention sessions consisted of a mixture of circuits and games-based activities designed to elicit a heart rate of $>90 \%$ Heart Rate maximum $\left(\mathrm{HR}_{\max }\right)$, with a $1: 1$ exercise to rest ratio (Hood et al., 2011). Throughout each exercise session, participants' HR were continuously monitored (Activio AB, Stockholm, SWE). Maximal HR was predicted using the formula developed by Tanaka et al. (2001), which has been validated for use in children and adolescents (Mahon et al., 2010). The intervention was delivered by a trained professional from Commando Joe’s ${ }^{\circledR}$ (Manchester, UK). Participants in the control group engaged in their usual day-to-day activities.

\subsubsection{Procedures}

The intervention and control groups were assessed at four time-points: baseline, midintervention, post-intervention and at a 3-month follow-up. Participants were asked to attend the laboratory at the same time of day ( $\pm 2 \mathrm{hrs}$ ) four times at each of the time-points, separated by a minimum of 24 hours, in a rested and fully hydrated state, at least two hours postprandial. 


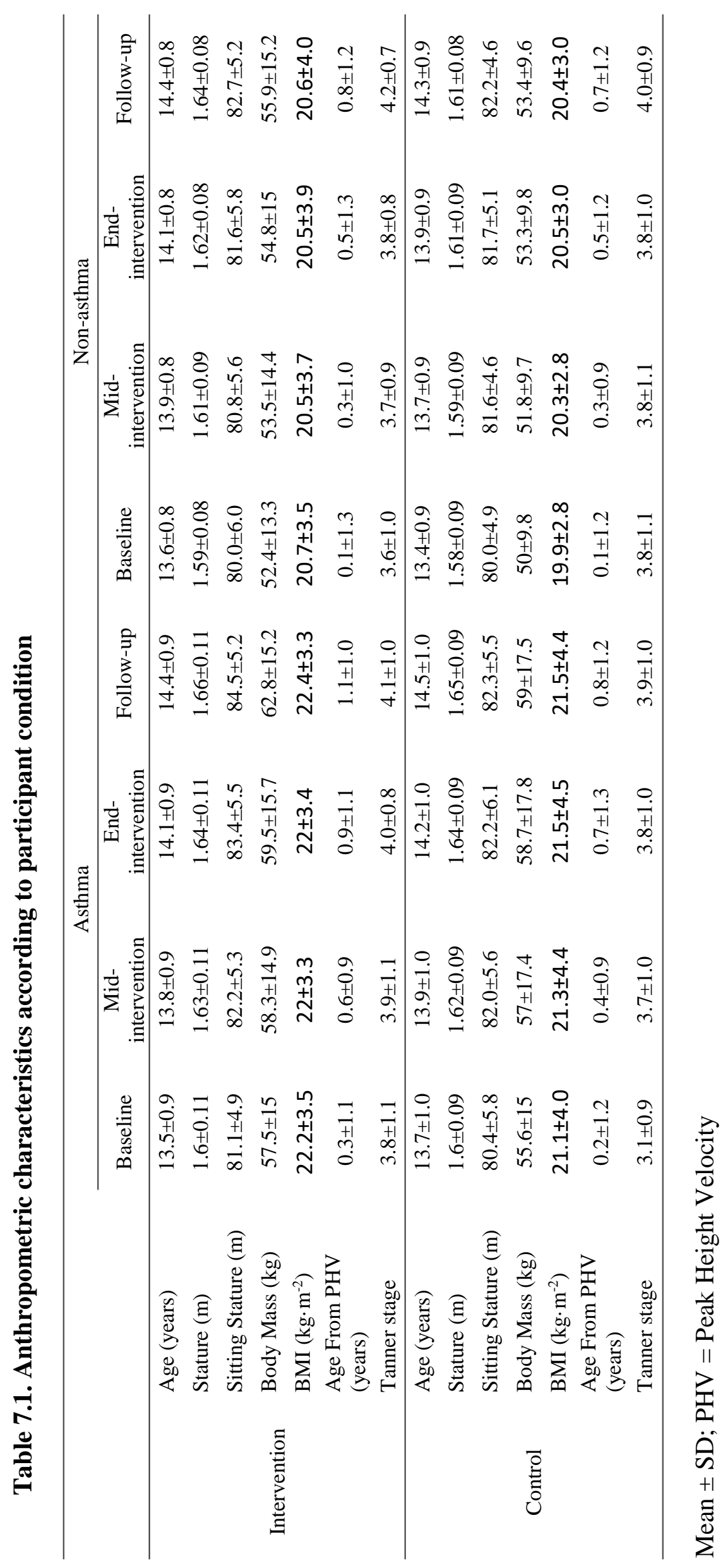




\subsubsection{Anthropometrics}

Body mass and stature were measured according to the techniques outlined by the International Society for the Advancement of Kinanthropometry (Stewart et al., 2011). Stature and sitting stature were measured to the nearest $0.1 \mathrm{~cm}$ (Seca213, Hamburg, Germany) and body mass to the nearest $0.1 \mathrm{~kg}$ (Seca876, Hamburg, Germany). Further, lower limb length was calculated as the difference between stature and sitting stature and then used to determine maturity offset using the equations of Mirwald et al. (2002).

\subsubsection{Incremental Test}

All exercise tests were performed on an electromagnetically braked cycle ergometer (Ergoselect 200, Ergoline GmbH, Lindenstrasse, Germany), with seat and handlebar height adjusted for each participant and kept consistent for all visits at a particular time-point. On the first visit, participants performed an incremental ramp test to volitional exhaustion to determine peak $\dot{V} \mathrm{O}_{2}$ and the Gas Exchange Threshold (GET). The ramp protocol consisted of 3 minutes of unloaded pedalling $(0 \mathrm{~W})$ followed immediately by an increase in work rate at $12-24 \mathrm{~W} \cdot \mathrm{min}^{-1}$. Throughout the test, participants were asked to maintain a cadence of $75 \pm 5$ revolutions per minute. The peak $\dot{V} \mathrm{O}_{2}$ was taken as the highest 10 -second average attained prior to exhaustion. The GET was determined using the V-slope method (Beaver et al., 1986). The work rate that would elicit $40 \%$ of the difference between GET and peak $\dot{V} \mathrm{O}_{2}(\Delta 40 \%)$ was subsequently determined (heavy-intensity), accounting for the mean response time for $\dot{V} \mathrm{O}_{2}$ during ramp exercise (i.e., two thirds of the ramp rate was deducted from the work rate at the GET and peak (Whipp et al., 1981)).

\subsubsection{Step Exercise Tests}

The subsequent three visits enabled the determination of $\dot{V} \mathrm{O}_{2}, \mathrm{HR}$ and deoxyhaemoglobin kinetics using heavy-intensity Constant Work Rate (CWR) tests, comprising of six minutes of pedalling with no external resistance followed by an abrupt transition to the target work rate, which was maintained for six minutes. The participants were asked to maintain a cadence of $75 \pm 5$ revolutions per minute throughout the tests. 


\subsubsection{Measurements}

Pulmonary ventilation (VE) and gas exchange $\left(\dot{V} \mathrm{O}_{2}\right.$ and $\left.\dot{V} C \mathrm{O}_{2}\right)$ were measured on a breath-by-breath basis (Jaeger Oxycon Mobile, Jaeger, Hoechberg, Germany) using a facemask with low dead-space connected via an impeller turbine assembly (Jaeger Triple V, Hoechberg, Germany). Gas analysers were calibrated prior to each test with gases of known concentrations and the turbine volume transducer was calibrated using a built-in function calibrated using a 31 syringe (Hans Rudolph, Kansas City, MO). The volume and concentration signals were time-aligned by accounting for the delay in capillary gas transit and analyser rise time relative to the volume signal. The inspired and expired gas volumes and concentration signals were continuously sampled at $100 \mathrm{~Hz}$. Heart rate was recorded continuously at a sampling frequency of 250Hz (Physio Flow PF-05 Lab1, Manatec Biomedical, France).

The oxygenation status of the right m.vastus lateralis was also monitored during each CWR test using near-infrared spectroscopy (Portamon, Artinis Medical Systems, Netherlands). The Portamon device consisted of three light sources emitting two wavelengths (760 and $850 \mathrm{~nm}$ ) and a photon detector. The reflected light was recorded continuously at $10 \mathrm{~Hz}$ and used to estimate the changes in concentration of oxygenated, deoxygenated $([\mathrm{HHb}])$ and total haemoglobin. The Portamon device was placed at the midpoint of the muscle using micropore tape (3M, Maplewood, $\mathrm{MN}$ ); to minimise movement and the interference of extraneous light, a bandage was wrapped around the Portamon device and leg. In order to account for adiposity, BMI was calculated, as a change in adiposity can affect the NIRS light source effectively reducing the distance the light can travel into the muscle.

\subsubsection{Data Analysis}

To account for body size, analysis of covariance (ANCOVA) was used to determine the allometric relationship between peak $\dot{V} \mathrm{O}_{2}$ and body mass using log transformed data. Common allometric exponents were confirmed for the data and power function ratios $\left(\mathrm{Y} / \mathrm{X}^{\mathrm{b}}\right)$ were computed. 


\subsubsection{Oxygen Uptake Kinetic Analysis}

Breath-by-breath $\dot{V} \mathrm{O}_{2}$ responses were first examined using a 5-second moving average to identify and remove any errant breaths caused by coughing, swallowing, sighing, for example, which were more than four standard deviations from the local mean. Each transition was then interpolated to 1-second intervals, time-aligned to the start of exercise and averaged to produce a single response profile for each timepoint. Each CWR profile was then corrected for baseline $\dot{V} \mathrm{O}_{2}$ and a monoexponential model applied (Equation 7.1):

Equation 7.1

$$
Y_{(t)}=A_{1}\left(1-e^{-\left(t-\delta_{1}\right) / \tau_{1}}\right)
$$

Equation 7.2

$$
Y_{(t)}=A_{1}\left(1-e^{-(t) / \tau_{1}}\right)
$$

where $\mathrm{Y}$ is the increase in the parameter $\left(\dot{V} \mathrm{O}_{2},[\mathrm{HHb}]\right.$ and $\left.\mathrm{HR}\right)$ at time $t$ above the baseline value (calculated as the mean of the first 45-seconds of the last minute of baseline pedalling). $\mathrm{A}_{1}, \delta_{1}$ and $\tau_{1}$ are the primary component amplitude, time delay (which was allowed to vary freely), and time constant, respectively. Variables derived from the mono-exponential $\left(\mathrm{A}_{1}, \delta_{1}\right.$ and $\left.\tau_{1}\right)$ and their $95 \%$ confidence intervals were determined by least squares non-linear regression analysis (Graphpad Prism, Graphpad Software, San Diego, CA). A mono-exponential model was selected as a bi-exponential model was found to produce an ambiguous fit to the data. Purpose-designed custom software was then used to iteratively fit a singleexponential function to the $\dot{V} \mathrm{O}_{2}$ data until the window encompassed the entire exercise response. The resulting phase II time constants were plotted against time to identify the point at which the phase II time constant consistently deviated from the previously "flat" profile providing the start time of the slow component (Fawkner \& Armstrong, 2004b). The amplitude of the $\dot{V} \mathrm{O}_{2}$ slow component was determined as the difference between the $\dot{V} \mathrm{O}_{2}$ at end of phase II and at end exercise $(\mathrm{t}=360)$ and 
presented in absolute terms and as a percentage of end exercise $\dot{V} \mathrm{O}_{2}$. Finally, the mean response time (MRT) was calculated by fitting Equation 7.2, from $\mathrm{t}=0$ to $\mathrm{t}=$ 360.

\subsubsection{2 [HHb] \& Heart Rate Kinetics Analysis}

The $[\mathrm{HHb}]$ and $\mathrm{HR}$ responses to the CWR tests were modelled using a monoexponential function. Each transition was interpolated to 1-second intervals, timealigned to the start of exercise and averaged to produce a single response profile for each measurement time-point. The $[\mathrm{HHb}]$ data were baseline averaged, expressed as a percentage of end exercise amplitude and then averaged into 5-second time bins. The $[\mathrm{HHb}$ ] was modelled using Equation 7.1 with the time delay identified as the time after exercise onset at which $[\mathrm{HHb}]$ began a systematic increase above the nadir value (Murias et al., 2016). The mono-exponential function was fitted between the identified time delay and time at which end of phase II was identified by the monoexponential model of the $\dot{V} \mathrm{O}_{2}$ kinetics. The HR response was modelled by both Equations 7.1 and 7.2, as per previous research (McNarry et al., 2017, Murias et al., 2010); however, Equation 7.1 was selected for analysis as it was deemed the superior fit for $91 \%$ of transitions (Graphpad Prism, Graphpad Software, San Diego, CA). The model was fitted between $\mathrm{t}=0$ and $\mathrm{t}=360$. The $[\mathrm{HHb}]$ time delay and time constant were subsequently summed to give the MRT. Additionally, to assess the fractional $\mathrm{O}_{2}$ extraction and $\mathrm{O}_{2}$ delivery, the $[\mathrm{HHb}] / \dot{V} \mathrm{O}_{2}$ ratio was calculated (Murias et al., 2011). Both [HHb] and $\dot{V} \mathrm{O}_{2}$ data were normalised for each participant, with the $\dot{V} \mathrm{O}_{2}$ left-shifted by $15 \mathrm{~s}$ to account for the cardiodynamic phase (Murias et al., 2010). Values found to be greater than 1.0 reflect a greater reliance on fractional $\mathrm{O}_{2}$ extraction and a poorer local $\mathrm{O}_{2}$ delivery relative to muscle $\mathrm{O}_{2}$ utilisation compared with the exercise steady-state (values $=1.0$ ). Each of the data sets were then averaged into $5 \mathrm{~s}$ time bins and the ratio of $[\mathrm{HHb}]: \dot{V} \mathrm{O}_{2}$ calculated for each time bin. Subsequently, the average of all the individual time bins was calculated to produce an overall "mean" ratio.

\subsubsection{Statistics}

The data were normally distributed, assessed by the Shapiro-Wilk test. To assess the influence of asthma, the intervention and their interaction, a mixed model ANOVA 
was used with subsequent Tukey's post-hoc comparisons when significant main effects were found. Pearson's correlation coefficients were used to investigate the degree of association between key variables.

All analyses were conducted using the intention-to-treat approach, including all participants with measures at all time-points. Furthermore, data were subsequently analysed using a sensitivity analysis on the participants who participated in the majority of the intervention sessions $(>70 \%)$. Eta-squared $\left(\eta_{p}^{2}\right)$ effect size was calculated from baseline to follow-up.

All statistical analyses were conducted using SPSS v22 (IBM Corp, Armonk, NY). All data are presented as means \pm standard deviation (SD). Statistical significance was accepted when $\mathrm{P}<0.05$.

\subsection{Results}

Participants' maturity increased throughout the study, with increases in stature and body mass observed in each group, a full breakdown of participants' descriptive statistics is shown in Table 7.1. The participants with asthma in the intervention group consisted of $87 \%$ with mild persistent and $13 \%$ with moderate or severe asthma, whilst the participants with asthma in the control group consisted of $77 \%$ and $23 \%$, in these groups respectively. This prevalence was similar in both the intention-to-treat and sensitivity analyses. Where no difference between intention-totreat and sensitivity analysis was found, results refer to the former.

\subsubsection{Incremental Ramp Exercise}

There was a $28 \%$ drop-out rate for the incremental ramp measures, however this includes $15 \%$ who missed one time-point due to external factors. There was no significant difference between the intervention and control groups or asthma and non-asthma participants at baseline (Table 7.2). The intervention was associated with a significant increase in peak $\dot{V} \mathrm{O}_{2}$ at post-intervention, irrespective of asthma status, which returned to baseline at follow-up. No significant changes in peak $\dot{V} \mathrm{O}_{2}$ were found in the control groups with time or between those with and without asthma at 
any time-point. These differences were maintained when scaled for body size, with a higher scaled peak $\dot{V} \mathrm{O}_{2}$ at post-intervention in the intervention groups but no change in control groups.

There was no difference in the GET between those with and without asthma. Irrespective of treatment group, the GET significantly increased with time. However, sensitivity analysis revealed the GET did not significantly increase over the intervention, regardless of asthma status. In agreement, when expressed relative to peak $\dot{V} \mathrm{O}_{2}\left(\mathrm{GET} \% \dot{V} \mathrm{O}_{2}\right)$, there was no difference in relative GET between groups.

\subsubsection{Constant Work Rate Exercise}

At baseline, participants with and without asthma did not significantly differ in any of the parameters of the dynamic $\dot{V} \mathrm{O}_{2}$ (Figure $7.1 \& 7.2$ ), HR or [HHb] response (Tables $7.2 \& 7.3$ ). The phase II amplitude significantly increased throughout the intervention $\left(F(3,138)=18.9, \mathrm{P}<0.05 \eta_{\mathrm{p}}^{2}=0.29\right)$. Post-hoc analyses revealed that all groups, except the control asthma group, significantly increased the phase II amplitude between baseline, post-intervention and follow-up. A slow component was evident in all participants, but no effect of asthma or the intervention was manifest. A significant increase in MRT across time-points $(F(2.1,97)=6.6, \mathrm{P}<$ $0.05 \eta_{\mathrm{p}}{ }^{2}=0.13$ ) was found, with post-hoc analyses attributing differences between baseline and follow-up in participants with asthma in the intervention group. However, the sensitivity analysis found no differences in MRT across time in any group.

The HR kinetics showed a significant main effect for time for HR amplitude ( $F$ (3, $\left.132)=10.4, \mathrm{P}<0.05 \eta_{\mathrm{p}}{ }^{2}=0.19\right)$ and for group in both $\operatorname{HR}$ amplitude $(F(3,44)=$ 3.2, $\left.\mathrm{P}<0.05 \eta_{\mathrm{p}}{ }^{2}=0.17\right)$ and HR time constant $\left(F(3,40)=3.7, \mathrm{P}<0.05 \eta_{\mathrm{p}}{ }^{2}=0.22\right)$. Post-hoc analyses showed HR amplitude significantly increased from baseline to post-intervention in both intervention and control non-asthma groups, with a further increase at follow-up in the control non-asthma group. Further, HR amplitude was found to be significantly higher in non-asthma compared to asthma intervention groups at post-intervention. 


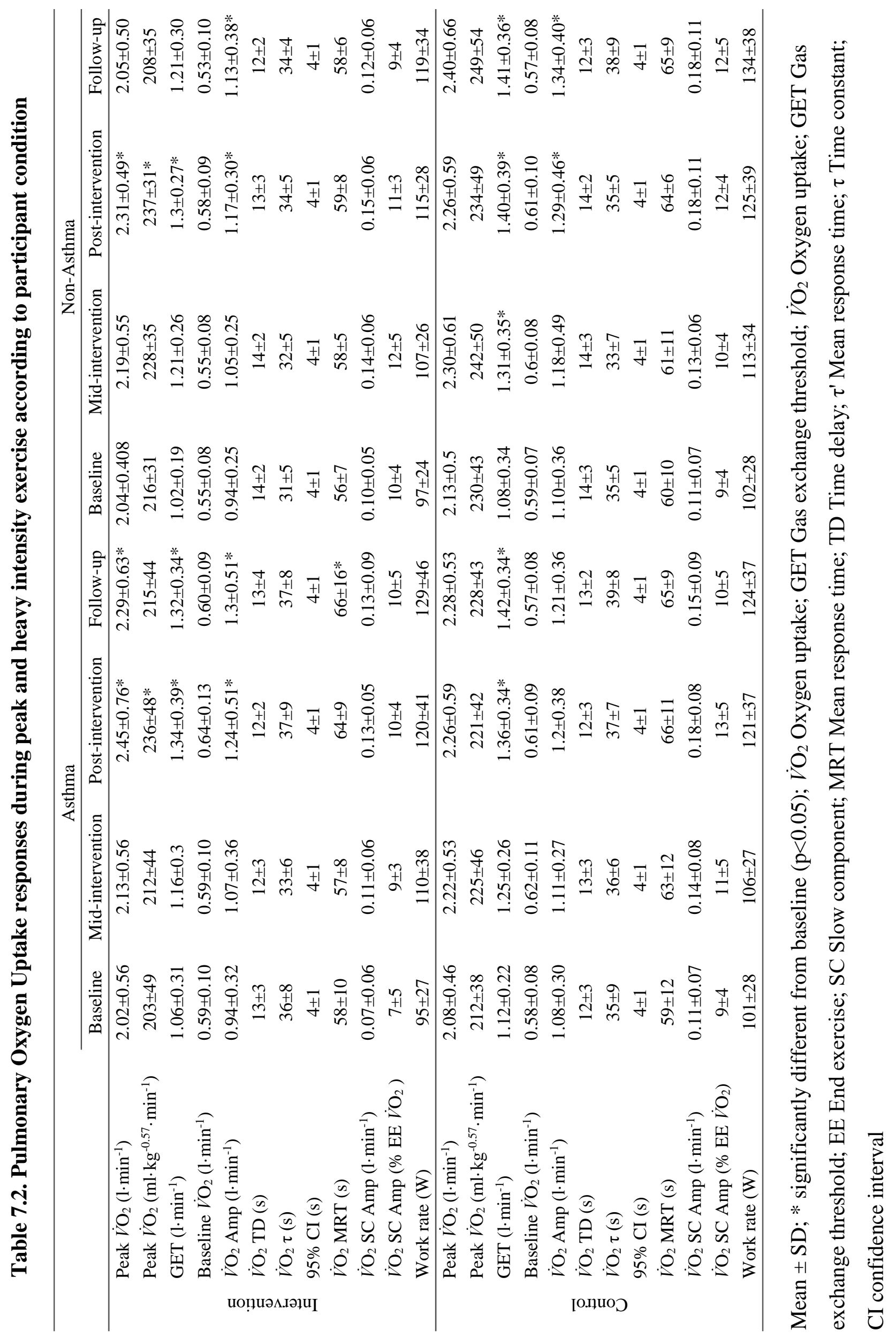




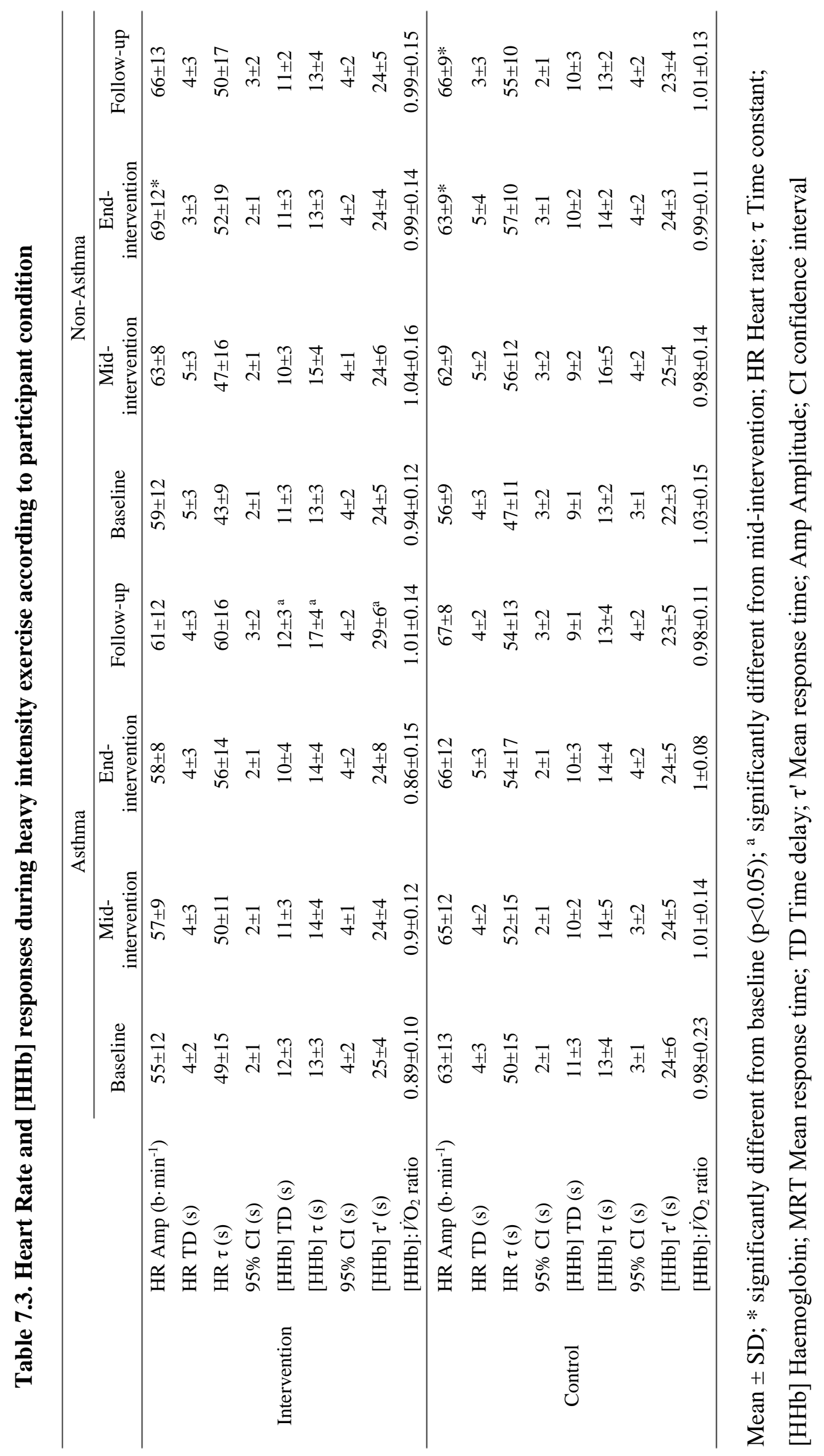




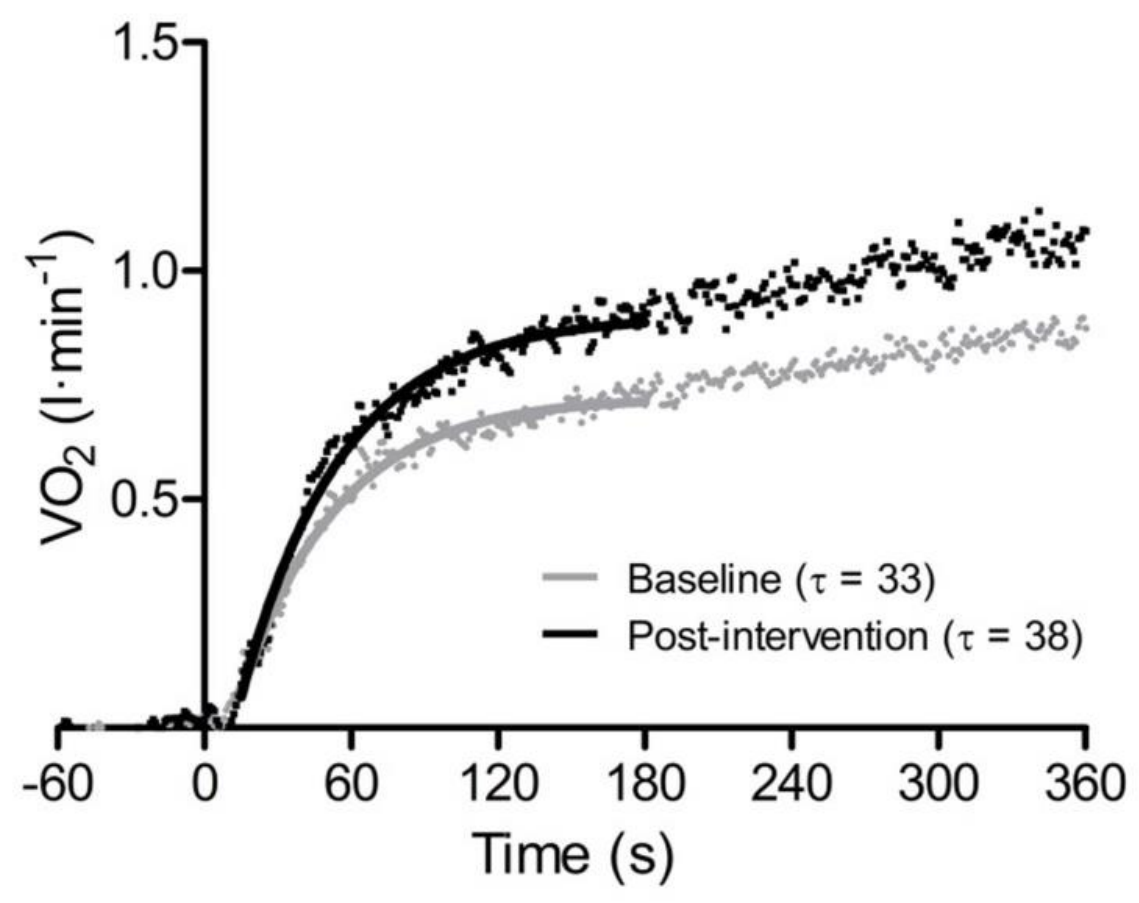

Figure 7.1. Pulmonary oxygen uptake response during heavy-intensity exercise in asthma

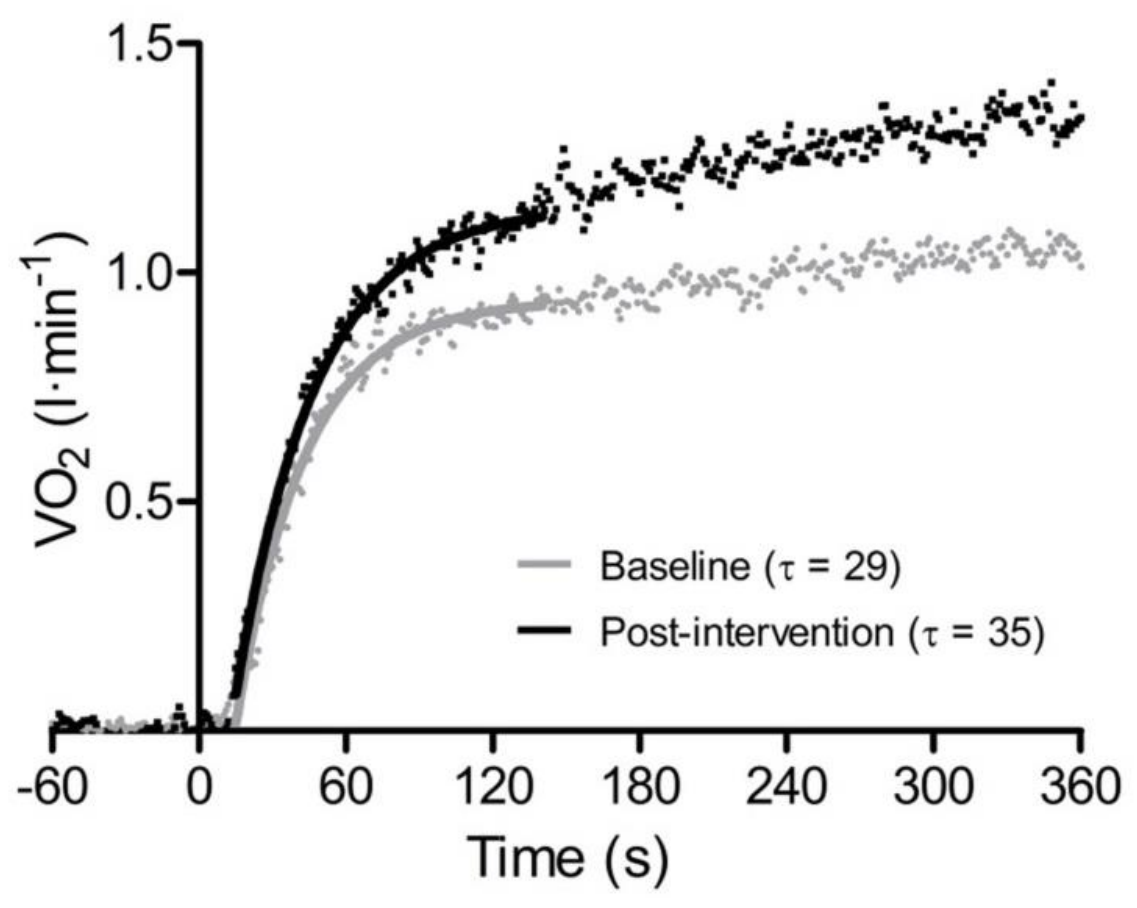

Figure 7.2. Pulmonary oxygen uptake response during heavy intensity exercise in non-asthma 
No changes in BMI occurred in the participants over the course of measures in either the intervention or control groups, therefore any changes observed in the [HHb] kinetics are not thought to be due to changes in adiposity. A significant main effect for time was found for the $[\mathrm{HHb}]$ time delay $\left(F(3,134)=3.1, \mathrm{P}<0.05 \eta_{\mathrm{p}}{ }^{2}=0.07\right)$, time constant $\left(F(3,132)=2.8, \mathrm{P}<0.05 \eta_{\mathrm{p}}{ }^{2}=0.06\right)$ and $\operatorname{MRT}(F(3,129)=1.9, \mathrm{P}<$ $\left.0.05 \eta_{\mathrm{p}}{ }^{2}=0.04\right)$, but not the overall $[\mathrm{HHb}]: \dot{V} \mathrm{O}_{2}$ ratio. Post-hoc analyses showed the $[\mathrm{HHb}] \tau$, time delay and $\tau^{\prime}$ to significantly increase between mid-intervention and follow-up in the intervention asthma group. Sensitivity analyses showed no significant difference between time-points or groups for the $[\mathrm{HHb}]$ time constant or the MRT. There were no differences between those with and without asthma at baseline for $[\mathrm{HHb}]: \dot{V} \mathrm{O}_{2}$. A transient overshoot in the $[\mathrm{HHb}]: \dot{V} \mathrm{O}_{2}$ response was observed following the step change in exercise demand, irrespective of group.

There were significant relationships at baseline between scaled peak $\dot{V} \mathrm{O}_{2}$ and $\dot{V} \mathrm{O}_{2} \tau$ ( $\mathrm{r}=-0.27 ; \mathrm{P}=0.03), \dot{V} \mathrm{O}_{2}$ amplitude $(\mathrm{r}=0.75 ; \mathrm{P}<0.05)$, slow component amplitude $(\mathrm{r}=0.46 ; \mathrm{P}<0.05), \mathrm{HR} \tau(\mathrm{r}=-0.39 ; \mathrm{P}<0.05)$ and $[\mathrm{HHb}] \tau(\mathrm{r}=-0.26 ; \mathrm{P}<0.05)$. At baseline $\dot{V} \mathrm{O}_{2}$, [HHb] and HR $\tau$ were not associated with any other parameters. Postintervention measures showed significant relationships between scaled $\dot{V} \mathrm{O}_{2}$ and $\dot{V} \mathrm{O}_{2}$ amplitude $(\mathrm{r}=0.87 ; \mathrm{P}<0.05)$, slow component amplitude $(\mathrm{r}=0.52 ; \mathrm{P}<0.05)$, and $[\mathrm{HHb}] \tau(\mathrm{r}=-0.39 ; \mathrm{P}<0.05)$. Post-intervention $\dot{V} \mathrm{O}_{2},[\mathrm{HHb}]$ and $\mathrm{HR} \tau$ were not associated with any other parameters.

\subsubsection{Intervention Intensity}

Throughout the intervention sessions, exclusive of warm-up and cool-down, participants' mean HR and mean HR peak were $155 \pm 16$ and $189 \pm 12$ beats. minute ${ }^{1}$, respectively $\left(78 \pm 8 \% \mathrm{HR}_{\max }\right.$ and $\left.95 \pm 6 \% \mathrm{HR}_{\max }\right)$. The HR exceeded the threshold of $>90 \% \mathrm{HR}_{\max }$ on average of $24 \%$ of the total time.

\subsection{Discussion}

This is the first study to investigate the influence of asthma, HIIT and their interaction on the dynamic pulmonary $\dot{V} \mathrm{O}_{2}$ response and its determinants, including HR and localised muscle fractional oxygen extraction. One of the main findings of 
the present study was that the $\dot{V} \mathrm{O}_{2}$ kinetics of adolescents with asthma were not significantly different from their healthy peers. The HIIT intervention, however, did not significantly improve the $\dot{V} \mathrm{O}_{2}$ kinetic response of the adolescents, irrespective of asthma, although it may be able to elicit significant improvements in peak $\dot{V} \mathrm{O}_{2}$. Taken together, these findings demonstrate that asthma does not limit the response to exercise or training. The findings also suggest that HIIT may not engender peripheral adaptations in muscles which are of a differing modality to that of the intervention, such as enhanced oxygen utilisation as reflected by the [HHb] response, in adolescents.

In contrast to the hypothesis and previous studies (Berntsen et al., 2009, Pianosi \& Davis, 2004), asthma did not affect peak $\dot{V} \mathrm{O}_{2}$ or $\dot{V} \mathrm{O}_{2}$ kinetics responses. Given that adolescents with other chronic airway conditions, such as Cystic Fibrosis, have been shown to have impaired $\dot{V} \mathrm{O}_{2}$ kinetics (Fielding et al., 2015, Saynor et al., 2016), it was anticipated that this would also be manifest in adolescents with asthma. This lack of effect could have been due to the current participants being predominantly characterised by mild asthma which may have been insufficient to cause derangements. Indeed, if participants administered their medication as prescribed, this could have potentially "normalised" these adolescents in comparison to their healthy peers; as taking their medication should control exercise induced symptoms of asthma (Minic \& Sovtic, 2017). It is perhaps pertinent to note, however, that even in those with mild asthma, a lower peak $\dot{V} \mathrm{O}_{2}$ has previously been reported compared to their healthy age- and sex-matched counterparts (Clark \& Cochrane, 1988, Garfinkel et al., 1992, Vahlkvist \& Pedersen, 2009). Furthermore, in agreement with other studies in children (Breese et al., 2010, Fawkner \& Armstrong, 2004a, McNarry et al., 2015b), there was no correlation between peak $\dot{V} \mathrm{O}_{2}$ and the $\dot{V} \mathrm{O}_{2}$ kinetic time constant, questioning the applicability of findings with regards to peak $\dot{V} \mathrm{O}_{2}$ to the dynamic $\dot{V} \mathrm{O}_{2}$ response. Additionally, the absence of influence of asthma may be related to the derangements associated with asthma taking longer to manifest. The current findings therefore suggest that there is no deconditioning effect of asthma on the dynamic $\dot{V} \mathrm{O}_{2}$ kinetics response in adolescents.

The HIIT intervention in the present study elicited a significant increase in peak $\dot{V} \mathrm{O}_{2}$, but no changes were observed in the $\dot{V} \mathrm{O}_{2}$ kinetics, in agreement with previous 
findings in healthy children (Lambrick et al., 2016), but contradicting findings in adults (McKay et al., 2009, Raleigh et al., 2016) who undertook moderate-intensity step tests. Whilst this failure to elicit significant adaptations in the dynamic $\dot{V} \mathrm{O}_{2}$ response could question the applicability of heavy-intensity $\dot{V} \mathrm{O}_{2}$ kinetics in youth to assess the influence of HIIT interventions, this may also be attributable to a lack of commonality between the training and testing modalities. Specifically, whole-body HIIT exercises in the current study and that of McNarry et al. (2015b) were assessed via cycle and treadmill ergometry, respectively, whereas a cycle ergometer was used for both training and testing in previous studies that have reported significant improvements (McKay et al., 2009, Raleigh et al., 2016). This apparent importance of exercise modality may be related to the relative contribution of central and peripheral adaptations to training-induced increases (Poole et al., 2008); peripheral adaptations suggested to be predominantly elicited by HIIT could have been manifest in muscles not substantially utilised during cycle ergometry, masking the effect of the training intervention. Indeed, the differential effect of HIIT on the central and peripheral determinants of aerobic fitness may, at least in part, explain the dissociation between peak $\dot{V} \mathrm{O}_{2}$ and $\dot{V} \mathrm{O}_{2}$ kinetics in the present study. This finding agrees with suggestions that, while a higher peak $\dot{V} \mathrm{O}_{2}$ is usually associated with faster $\dot{V} \mathrm{O}_{2}$ kinetics, the speed and amplitude of $\dot{V} \mathrm{O}_{2}$ kinetics are dissociated from peak $\dot{V} \mathrm{O}_{2}$ (Poole \& Jones, 2012). Finally, when interpreting the present results it is important to note that, converse to some (Christensen et al., 2016) but not all (McNarry et al., 2015b) previous studies reporting a significant effect of HIIT, the target work rate was reassessed at each time-point, potentially normalising any speeding in the $\dot{V} \mathrm{O}_{2}$ kinetics response. Indeed, a greater phase II amplitude following HIIT was observed in the present study, implying participants were achieving a greater absolute response in the same time.

The dissociation between peak $\dot{V} \mathrm{O}_{2}$ and $\dot{V} \mathrm{O}_{2}$ kinetics and the lack of effect of HIIT may partially be explained by increases in maturity from baseline to follow-up. Specifically, in a two year longitudinal study, the $\dot{V} \mathrm{O}_{2}$ kinetics of healthy children were found to be characterised by a slowed time constant and increased $\dot{V} \mathrm{O}_{2}$ slow component, which may be related to a reduced exercise tolerance (Breese et al., 2010). It may therefore be suggested that the HIIT intervention was able to 
counteract the slowing of $\dot{V} \mathrm{O}_{2}$ kinetics associated with increased maturity. Additionally, or perhaps alternatively, adolescents' baseline fitness may have been an influential factor in determining the response to training. However, whilst there is a strong relationship between baseline peak $\dot{V} \mathrm{O}_{2}$ and the degree of change (Eliakim et al., 1996, Mandigout et al., 2001), there is a paucity of research in relation to the $\dot{V} \mathrm{O}_{2}$ kinetics response. Moreover, the baseline $\dot{V} \mathrm{O}_{2}$ time constants observed in the current study, which are typical for their age group, are faster than usually reported in healthy, untrained adults (Armstong \& McNarry, 2016), thereby potentially limiting the capacity for improvement.

The lack of favourable adaptations elicited by the HIIT intervention in the $\dot{V} \mathrm{O}_{2}$ kinetic response is reflected in the lack of effect on the HR or [HHb] kinetics, which broadly reflect bulk oxygen delivery (MacPhee et al., 2005) and local fractional $\mathrm{O}_{2}$ extraction (DeLorey et al., 2007, Grassi et al., 2003), respectively. Interestingly, there was a slowing of the $[\mathrm{HHb}]$ response, which was significant in the asthma intervention group at follow-up. This may indicate that the age-related slowing in $\dot{V} \mathrm{O}_{2}$ kinetics congruent with previous research (Breese et al., 2010) may be attributable to an altered muscle $\mathrm{O}_{2}$ extraction. Indeed it could be postulated that such a decrease in oxidative capacity is a consequence of the increased glycolytic capacity typically observed with growth and maturation (Kriketos et al., 1997). The lack of effect of time or intervention on the HR time constant is consistent with previous research which purports that the $\dot{V} \mathrm{O}_{2}$ kinetic response is not limited by $\mathrm{O}_{2}$ delivery in healthy populations during upright exercise (Poole \& Jones, 2012).

Fundamental to the definition of HIIT is the use of a high exercise intensity throughout the intervals to engender a significant physiological response. In accord with recent reviews (Costigan et al., 2015, Eddolls et al., 2017), the target intensity in the current study was $>90 \% \mathrm{HR}_{\max }$ (Hood et al., 2011), but the continuous HR data collected demonstrated that the participants typically averaged $\sim 80 \% \mathrm{HR}_{\max }$. Whilst this is still above the threshold suggested to be associated with physiological gain (Baquet et al., 2003), the short bout duration and faster recovery of children's heart rates compared to adults (Falk \& Dotan, 2006) may have interacted to reduce the stimulus received. Specifically, children will have returned to nearer baseline values between each bout, thereby requiring a greater subsequent intensity or duration to 
increase their HR above the target $90 \% \mathrm{HR}_{\max }$ threshold. Indeed, a higher average HR was reported by Lambrick et al. (2016) who utilised longer bout durations. However, it is worth noting the significantly longer study duration of the current intervention relative to other studies (Baquet et al., 2001, Tjonna et al., 2009, Weston et al., 2016), which have typically used 2-3 month interventions. It is therefore likely that these short interventions will be associated with a higher average HR as the participants will only have to attend fewer sessions, perhaps giving more effort for each one. Further work is required to elucidate the optimal work to rest ratios and durations in children to enable the sustained attainment of the target HR.

The repeated transitions to determine the dynamic $\dot{V} \mathrm{O}_{2}, \mathrm{HR}$ and $[\mathrm{HHb}]$ responses at each time-point was a strength to the study which allowed confidence in the results, with $95 \%$ confidence intervals ( $<5$ seconds) well within those recommended by Fawkner and Armstrong (2003). Furthermore, whilst previous research has implemented HIIT interventions, no study has considered the sustainability of HIITinduced training adaptations through the inclusion of a 3-month follow-up. Nonetheless, the study is not without its limitations. Specifically, it is not possible to preclude the potential for a self-selection bias, whereby adolescents whom enjoyed exercise were more likely to volunteer to participate. However, the baseline peak $\dot{V} \mathrm{O}_{2}$ of the current participants does not suggest they were highly active or trained $\left(37-41 \mathrm{ml} \cdot \mathrm{min}^{-1} \cdot \mathrm{kg}^{-1}\right.$ vs. trained $=54 \mathrm{ml} \cdot \mathrm{min}^{-1} \cdot \mathrm{kg}^{-1}$, untrained $=43 \mathrm{ml} \cdot \mathrm{min}^{-1} \cdot \mathrm{kg}^{-1}$ Marwood et al., 2010). Although the age- and sex-matched controls should have accounted for differences in training effects, the sample size resulted in pooling boys and girls for analysis which may have reduced interpretation. Indeed, Lai et al. (2016) found that adolescents $\mathrm{HR}$ and $\dot{V} \mathrm{O}_{2}$ kinetics differ between sexes and therefore future studies should seek to recruit larger sample sizes to allow the independent analysis of boys and girls.

\subsection{Conclusion}

In conclusion, asthma does not deleteriously influence the cardiorespiratory fitness of adolescents, with a similar peak $\dot{V} \mathrm{O}_{2}$ and $\dot{V} \mathrm{O}_{2}$ kinetics at baseline, demonstrating the potential to integrate participants irrespective of condition into future exercise 
interventions. Moreover, the consistent significant increases in peak $\dot{V} \mathrm{O}_{2}$ irrespective of asthma status suggest that the magnitude of training-induced improvements in cardiorespiratory fitness are unaffected by asthma. Whilst the six-month HIIT intervention did not engender favourable adaptations in the dynamic $\dot{V} \mathrm{O}_{2}$ response, it may have attenuated the age-related decline typically reported, and therefore future research should evaluate the differences pre- and post-puberty. 


\section{Thesis study map}

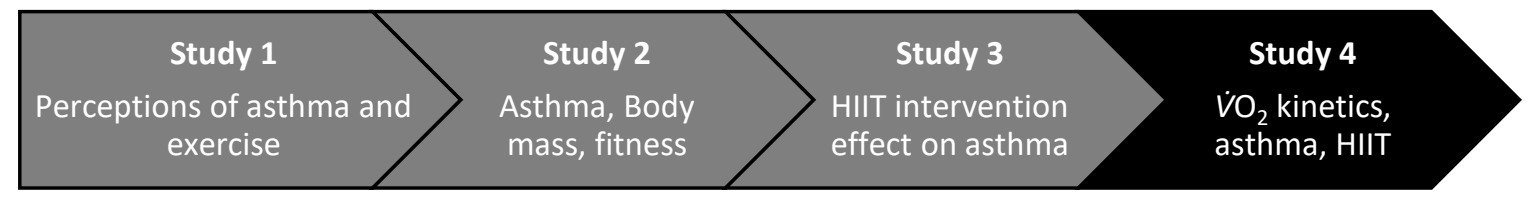

\section{Chapter 7}

Aims:

- To investigate the influence of asthma and HIIT, and their interaction, on the dynamic $\dot{V} \mathrm{O}_{2}$ response in adolescents

Key findings for future chapters

- Asthma does not deleteriously influence the $\dot{V} \mathrm{O}_{2}$ response to an instantaneous change in the metabolic demand

- HIIT did not elicit any significant improvements in the $\dot{V} \mathrm{O}_{2}$ kinetics irrespective of asthma but may be able to attenuate the age-related decline 
CHAPTER 8 SYNTHESIS 


\section{Chapter 8: Synthesis}

The importance of exercise and physical activity for adolescents with asthma is wellrecognised but little was previously known about the influence of a specific exercise intervention in those with asthma and its ability to reduce asthma symptoms. The purpose of the thesis was therefore to ascertain the perceptions of not only adolescents with asthma but their healthy peers of asthma and those affected by its ability to exercise in order to co-develop, implement and evaluate a HIIT intervention to improve asthma symptoms and quality of life. The current chapter will synthesise this research, considering the findings of each of the studies and their implications as a whole.

\subsection{General discussion}

\subsubsection{Perception vs. ability of adolescents with asthma}

Participants with asthma in study 1 (Chapter 4) perceived themselves to be limited during exercise by their condition and to be less fit than their peers. Some of the adolescents with asthma were unable to keep up with their friends and would regularly sit out of physical education lessons and other sporting activities. Restriction due to asthma in physical education lessons and during social time with peers led to participants feeling isolated as they were unable to keep up. As those with asthma would sit out or stop exercising, occasionally using it as an excuse, contradicting previous research (Protudjer et al., 2012), adolescents without asthma developed the perception that their peers with asthma were less able than themselves.

Participants with asthma were found to be less fit than their healthy peers when considering the number of 20-metre shuttle runs completed, however, this measurement is often subject to significant inaccuracies dependent on selfmotivation and peer influence (Cairney et al., 2008). Moreover, participants with asthma were found to have a higher BMI than their peers and therefore, in combination with use of inhalers for fear of asthma attack (Protudjer et al., 2009), they may have been embarrassed to perform maximally in front of peers. The 20metre shuttle run test may therefore reflect a reinforced learning effect whereby 
many were repeatedly told by parents and carers to limit themselves, unnecessarily, due to their condition (Callery et al., 2003, Dantas et al., 2014). It is, however, important to note that this would not have applied to all those with asthma as some in the present study were found to be competitively motivated to show that their condition does not limit them (Chapter 4). Indeed, the results from study 3 and 4 (Chapter 6 and 7) indicate that this fear of asthma attacks was unwarranted as the adolescents with asthma tolerated the exercise programme well with no asthma attacks during any of the HIIT intervention sessions or physiological testing. Furthermore, no differences between those with and without asthma have been found in any of the four parameters of aerobic fitness (Whipp et al., 1981). From these findings, adolescents with asthma - albeit mostly mild asthma - are as fit as their peers (Chapter 5) and are able to adhere and improve their fitness suggesting the condition does not affect trainability (Chapter 6 and 7).

When sitting out of physical education, or when unable to "keep up" with their peers, adolescents with asthma miss out on the benefits of the exercise. Whether the selfexclusion is an excuse to avoid exercising or a fear of asthma attack and having to use their inhaler in front of their peers, not engaging in exercise may increase their symptoms and decrease their control over a prolonged period, potentially reducing their fitness (Lang et al., 2004, Vahlkvist et al., 2010). If adolescents with asthma are having to sit out of exercise then their control is not optimal as they should be able to participate in exercise (Berntsen, 2011). One potential factor behind adolescents' withdrawal from exercise is the misinterpretation of the normal exercise response in healthy children, such as increased breathing rate, heart rate and sweating (Rietveld et al., 2010, Williams et al., 2010). Adolescents with asthma may perceive these physiological responses as asthma symptoms and therefore withdraw from exercise to avoid an asthma attack. Continued participation in exercise would be a potential cure for this as they would learn to distinguish between normal exercise exertion and asthma symptoms.

A healthcare utilisation questionnaire (Appendix C) was developed as part of the study to assess the burden of the participants' asthma including medication use. The questionnaire revealed that adherence to medication was indeed an issue, with some participants reporting "taken when needed" with regards to their preventer inhaler 
that should be taken daily. Therefore, these participants would not have experienced the full benefit of their treatment (Chapman et al., 2017), resulting in poorer control. The questionnaire was poorly answered in general (with both missing names and amounts of medication prescribed) and the results were somewhat subjective (with regard to categorising the participants as mild/moderate or severe), however, it is important to identify people at risk of non-adherence in future studies as it can be a predictor of poor control (Price et al., 2015) and asthma exacerbations (Murphy et al., 2012, Williams et al., 2011). If these adolescents with asthma were to increase their fitness, which is possible as evidenced by studies 3 and 4 (Chapter 6 and 7) showing no difference between them and their healthy peers' trainability, their normal exercise exertion characteristics would reduce (Gutin et al., 2002). If, however, exercise provokes their asthma symptoms, then their asthma management needs amendment, as well-controlled asthma should not affect a person's ability to participate in exercise (Berntsen, 2011). Furthermore, adolescents may not be adhering to their prescribed medication, as evidenced by the healthcare utilisation questionnaire, potentially assuming that their asthma has been cured due a lack of symptoms in recent months, which may put them at a higher risk of an attack (Chapman et al., 2017).

Withdrawing from exercise as it is perceived as a trigger for asthma symptoms may put those adolescents at an increased risk of becoming obese (Hallstrand et al., 2000). Not only do adolescents with asthma avoid exercise (Chapter 4), moderate-tovigorous physical activity (MVPA) is also lower in these participants as shown in study 2 (Chapter 5). Exercise avoidance in combination with lower levels of MVPA may contribute to the increased BMI of participants with asthma observed in study 2 (Chapter 5). The asthma obesity phenotype directionality is not currently known, however, the findings from the study suggest fear of asthma attack may promote the development of obesity, although this does not rule out the bidirectional theory. The results from this thesis suggest that, with well controlled asthma, there should be no difficulty in participating in physical activity or exercise which may subsequently aid in weight management and possibly reduce symptoms and improve quality of life, as evidenced by previous studies (Ram et al., 2000). 


\subsubsection{Intervention evaluation}

In the following section, the HIIT intervention will be broken down and different elements will be discussed. Specifically, elements shown to have worked and strengthened the study as well as those which did not function as planned will be discussed, as these may aid in guidance for further work.

Although the intervention was informed by both study 1 (Chapter 4) and systematic reviews by Eddolls et al. (2017) and Wanrooij et al. (2014), the adherence was poor. Indeed, of the 221 adolescents to sign up for the intervention, only 44 were deemed to have attended enough of the sessions ( $>70 \%$ of the sessions) to be included within the sensitivity analysis. It is postulated that the lack of attendance was due to the sessions taking place either before or after school. Participants articulated that they had other commitments, ranging from school sport to being young carers, or that they just didn't want to get out of bed earlier to participate. Previous school-based interventions have used physical education lessons to run HIIT sessions (Costigan et al., 2016, Weston et al., 2016). However, when the school involved was approached and running the intervention during lesson time was suggested, the school were unwilling due to curriculum requirements. An additional benefit of this approach would have been the inherent separating of age groups as having mixed ages was cited as a barrier to exercise for some participants. It is worth noting that those who participated throughout highlighted the good relationships they developed with their peers in mixed age groups (information sought through informal discussions with the adolescents after the sessions) which could aid in personal development. Other time slots were considered in the design of the intervention, such as form period, break and lunch, however, not only did the head teacher not want the pupils' free time taken up, the length of time would not have been sufficient. Nonetheless, given a recent meta-analysis purporting the efficacy of extremely low doses of HIIT (Weston et al., 2014b), future studies could seek to incorporate shorter sessions into a physical education lessons without too much disruption. 
The sessions were implemented by an external facilitator from Commando Joe's ${ }^{\circledR}$ for two reasons: i) the instructor had professional training working with and implementing exercise interventions with children and adolescents and ii) for pragmatic reasons as due to the time consuming nature of collecting measures from participants within both the intervention and control schools, the research team was not always available to attend the intervention sessions. Potential issues do arise when using an external facilitator such as their motivation and scientific rigor. The sessions, however, were delivered as intended, starting with shorter exercise to rest periods of 10 seconds and increasing to 30 seconds over the first few weeks, in line with previous HIIT intervention exercise to rest ratios (Costigan et al., 2016). The exercise sessions were varied each day, informed by suggestions from study 1 (Chapter 4), and the facilitator was able to adapt each exercise to suit the ability of all participants. The sessions were, however, disrupted by some participants (arguing with each other or shouting at/not listening to the instructor) and therefore in order to keep the sessions running smoothly, discipline was required which extended some of the rest periods. The intervention was also possibly hindered by the number who attended in the first few sessions as there was overcrowding and therefore encouragement based on the real time monitoring of heart rate was reduced. The overcrowding, due to the school moving the session from their larger sports hall to their small gymnasium due to a schedule clash the teachers did not make us aware of, at the start of the intervention also likely exacerbated dropout rates as the sessions were required to be more regimented to stick to the prescribed exercise intensities.

The intervention only elicited an average of $\sim 80 \%$ heart rate maximum over the exercise session, which, whilst still above the threshold for physiological gain (Baquet et al., 2003), fell short of the target of $>90 \%$ heart rate maximum. It is pertinent to note, however, that this average includes the rest periods; in addition, due to the vigorous nature of the intervention, the belts would shift resulting in a loss of signal (which registered results which began at zero and gradually increased to their actual heart rate through averaging) and therefore the actual heart rate achieved during the exercise bouts is likely to be significantly higher. Future studies should consider methods to more accurately determine the actual exercise intensity during 
each exercise session to allow the dose-response relationship to be elucidated between HIIT and physiological adaptation in adolescents with and without asthma.

The length of the intervention may also have been associated with decreased enjoyment with time, despite the attempts to ensure varied exercise from one session to the next. The variety of exercises made it a more inclusive intervention due to different exercises appealing to different individuals. Although the specific exercises were based around the formative research in study 1 (Chapter 4$)$, the time of year at which this was conducted limited the applicability of some suggestions, such as exercising outside due to the temperature being far below that experienced during the summer term. Moreover, both the before and after school sessions were affected by the lack of daylight hours as the majority of participants walked to school. A thorough process evaluation was beyond the scope of this thesis but should be incorporated into future interventions to ensure lessons are learnt and fed-forward for subsequent studies.

\subsubsection{Effect of HIIT on asthma, fitness, obesity and physical activity}

This thesis provides an insight into the effect of high-intensity interval training (HIIT) on adolescents with asthma. Study 1 (Chapter 4) highlighted that although adolescents with asthma fear asthma attacks caused by physical activity, exercise was still their favourite activity to participate in. There was also a general perception that those with asthma participated in as much MVPA as their peers and could be as fit. However, study 2 (Chapter 5) showed that adolescents with asthma participated in significantly less MVPA in comparison to their healthy peers, consistent with previous work (Pianosi \& Davis, 2004, Vahlkvist \& Pedersen, 2009). In addition, participants with asthma had a significantly higher BMI than their peers with more overweight and obese adolescents in comparison, potentially due to the lack of physical activity (Walders-Abramson et al., 2009). Cardiorespiratory fitness, however, was shown to not be significantly different between those with and without asthma and was not different to other studies with untrained adolescents, as evidenced by the results in study 2, 3 and 4 (Chapter 5-7). Whilst the participants with asthma suffered characteristic impairments in their large $\left(\mathrm{FEV}_{1}, \mathrm{FEV}_{1} / \mathrm{FVC}\right)$ and small $\left(\mathrm{FEF}_{25-75}\right)$ airways, these did not translate into derangements in the $\mathrm{O}_{2}$ 
delivery pathway (Wasserman et al. 1994). Indeed, contrary to previous findings in pulmonary diseases (McNarry et al., 2017, Nery et al., 1982, Puente-Maestu et al., 2000, Saynor et al., 2016), this study did not find that $\dot{V} \mathrm{O}_{2}$ kinetics were deleteriously deranged by asthma. The lack of impairment in the $\dot{V} \mathrm{O}_{2}$ kinetics suggests that partially reduced lung function does not influence the $\mathrm{O}_{2}$ transport system in response to an instantaneous change in the metabolic demand. Moreover, as hypothesised there was no impairment in the $\mathrm{O}_{2}$ utilisation as assessed by [HHb] kinetics in adolescents with mostly mild severity of the disease and findings may be different in more severe populations.

Adolescents with asthma perceived that HIIT would be harder than those without asthma, as shown in Figure 4.3; however, the HIIT intervention was well tolerated as demonstrated by the absence of asthma attacks. As asthma medication was not withheld prior to or during exercise this may have aided in alleviating asthma symptoms, but confirms that as long as asthma is correctly controlled, there should be no issue in exercise participation (Minic \& Sovtic, 2017). Asthma-related quality of life was found to be significantly associated with cardiorespiratory fitness in study 2 (Chapter 5) and previous research (Fanelli et al., 2007), however, although increased fitness was observed throughout the intervention, an improvement in quality of life was not observed in study 3 (Chapter 6) or by others (Moreira et al., 2008). Interestingly, BMI did not improve and therefore, could be a reason for the disparity between fitness and quality of life. However, control participants' BMI did increase over the period of the intervention, with no reduction in quality of life. Moreover, the intervention participants at follow-up also had an increased BMI but no changes in quality of life. Therefore, it could be postulated that quality of life is based on the severity of asthma, rather than fitness or BMI; it was not possible to assess this due to challenges with grading severity through self-reported medication. Additionally, or alternatively, quality of life could be influenced by total physical activity which could not be tracked due to poor adherence and/or meeting the wear time criteria. It could, however, be hypothesised that external factors, such as school work, could have abolished any improvement in quality of life elicited by the intervention (McDonald, 2001). Indeed, measures at the mid-point and follow-up 
would have coincided with January or just followed summer exams which may have clouded our interpretation as to the effect of the intervention.

\subsection{Study strengths and weaknesses}

A great strength of this study is not only providing a comparison to a control asthma group, but also to their healthy peers, both in the intervention and control schools. This inclusive approach would have hopefully avoided any stigma associated with asthma through not singling out adolescents with the condition but incorporating them within a study of their peers. The inclusiveness of the study aided in codeveloping the intervention, which helped target exercise that both those with and without asthma would enjoy. Moreover, whilst the intervention was run by Commando Joes®, it was not laboratory based demonstrating that it can be implemented in a real world setting without any additional equipment, adhering to the protocol and exercises highlighted in Tables 6.2 and 6.3. A further strength of the thesis was the addition of more sensitive aerobic fitness parameters (gas exchange threshold, mean response time, gain and $\dot{V} \mathrm{O}_{2}$ kinetics), which have not previously been assessed in adolescents with asthma.

Nonetheless there are inherent limitations in this thesis which should be acknowledged. As the thesis was based around an exercise intervention, the potential for self-selection bias must be noted, as with any voluntary intervention. Adolescents with more severe asthma or exercise induced bronchoconstriction (EIB) may have not participated as it was found in study 1 (Chapter 4) that they often withdrew from exercise. It may question whether the study was able to target the correct group of adolescents with asthma as the asthma may have been so mild that no effect was there to be measured. However, measurable objective markers of asthma were present, namely evidence of airways inflammation and reduced lung function. Future studies could try to recruit participants with more severe asthma, although it is important to note that for the current inclusive study, it was not considered ethically sound to recruit and those with asthma to participate without voluntary participation. The selection bias is extended to the sub-sample measures of fitness as participants may have opted out of the measures due to their perception of strenuousness. 
However, as noted in study 3 (Chapter 6 ), the baseline measures of peak $\dot{V} \mathrm{O}_{2}$ of the participants did not suggest they were highly active or trained. Moreover, the differences in 20-metre shuttle run between those with and without asthma could suggest the selection bias for the incremental exercise test may have been more prominent in participants with asthma owing to the lack of difference between the two groups' peak $\dot{V} \mathrm{O}_{2}$ results. Due to the self-selection bias, the results from the current study may not be applicable to the general asthma population.

The power analysis used to determine sample size was originally calculated to detect a change in asthma-related quality of life, as outlined in the general methods section, and therefore due to the lack of sustained attendance at the intervention, the loss of power may have reduced the significance of the findings. In order to counteract any possible skewing of the data by use of intention-to-treat analysis, sensitivity analysis was used to confirm the findings. Due to the reduction in sample size when using sensitivity analysis, no differences were found when covarying for sex or maturation, potentially as the sample was required to be larger to power the tests. Finally the pooling of boys and girls for analysis may have reduced the interpretation of the findings; however this may have been mitigated in part by the age- and sex-matched controls.

It is also pertinent to note the limitations behind grading asthma severity by medication step as it is based on the assumption that participants are prescribed, and use, the medication appropriately (Global Initiative for Asthma, 2017, Taylor et al., 2008). According to the healthcare utilisation questionnaire (Appendix C), it was clear that participants with asthma were unaware of the type of medication that they were taking and whilst they knew how often their inhalers were prescribed to be taken, few adhered to this. Failing to take their medication as prescribed may have resulted in less controlled asthma than reported (Chapman et al., 2017), as evidenced by the high levels of FeNO in studies $2 \& 3$ (Chapter $5 \& 6$ ). Additionally, participants who reported more severe asthma by medication step and adhered to their medication may have better controlled their asthma, essentially cancelling out effects which would have been expected. This would explain observations when trying to covary for asthma severity which showed the more "severe" sufferers to have results more similar to healthy peers than those with "mild" asthma. However, 
these findings should be interpreted with caution as due to the sample size, splitting for severity potentially reduced the power of the statistics. In addition, due to the issues of assessing severity by medication step, the results are difficult to interpret. Although it has been documented that exercise can reduce severity (Andrade et al., 2014), it was unable to be assessed using the healthcare utilisation questionnaire as it was poorly answered and was therefore only able to gauge an overall severity rather than how this changed with time.

Although there was found to be no difference in fitness between those with and without asthma in this thesis, it may be speculated that because the intervention took place out of school hours; the more active, fitter adolescents could have been participating in competitive sport opting out of the intervention. Due to the perception that participants with asthma were limited by their condition (Chapter 4), even those with well-controlled asthma may not participate in school sport. Therefore, more conditioned asthma participants may be compared with healthy peers who were not trained. The use of objectively measured physical activity is essential when assessing an intervention, firstly to assess the compensation effect often observed in exercise interventions (Ridgers et al., 2018a) but also to assess the activity levels of the control participants. However, many of the participants were non-adherent and therefore the monitors were not worn or lost, hindering the results of study 2 (Chapter 5) and meaning that study 3 (Chapter 6) was unable to include the measure. It also meant that the activity level of the control participants was unknown and therefore they could have been more active than their intervention peers, masking the potential effect of the HIIT intervention.

Finally, the study also utilised the number of 20-metre shuttle runs used as an estimate of fitness, however the sub-sample measures were conducted on a cycle ergometer which may have resulted in a lack of commonality in the muscles used. The cycle ergometer was used for pragmatic reasons, such as ensuring that the exercise intensities were easily adjusted and for monitoring of heart rate which often dropped out using a treadmill. However, as the intervention consisted of body weight bearing land-based exercises, the ecological validity must be questioned, especially when assessing the $\dot{V} \mathrm{O}_{2}$ kinetics. 


\subsection{Implications for future research}

Although fear of an asthma attack was found to be a major barrier to exercise in adolescents with asthma, it was established that participating in exercise was one of the favourite activities, demonstrating promise for future inclusive exercise interventions. There is, however, a need for further education on the health benefits exercise provides for adolescents with asthma (Haughney et al., 2004). Indeed, as outlined in study 1 (Chapter 4 ), only $23 \%$ with and $18 \%$ without asthma were aware of such benefits of exercise. Further education could also be given to their healthy peers as a way to reduce stigma. Furthermore, adolescents with well-controlled mild asthma are able to participate in HIIT and be as fit as their peers. However, further research is required for adolescents with less-controlled, more severe asthma with regard to HIIT in addition to research in asthma to understand the optimal type, duration and frequency of exercise intervention.

The results of the thesis suggest that because adolescents with asthma "learn" they cannot participate in as much exercise as their peers (Chapter 4), it may lead to a sedentary lifestyle which carries forward into adulthood. Indeed, research in adults has shown asthma does have a deconditioning effect (Clark \& Cochrane, 1988), and therefore there is a need to ascertain at what age people with asthma begin to become less fit than their peers. There is therefore a need for further research into exercise interventions which experiment with type, duration and frequency of exercise to maintain participation through to adulthood. This may be accomplished with a doseresponse longitudinal study starting in adolescence, to find the exercise intervention requiring the least input to improve asthma-related quality of life through an increase in fitness to adulthood. Previously, higher intensity exercise has been found to be associated with the greatest improvements in fitness, asthma control and quality of life in children with asthma (Wanrooij et al., 2014) and as HIIT has been found to be more enjoyable and less time consuming than constant-intensity exercise (Costigan et al., 2015), it should appeal to the young adult.

Whilst the current study found HIIT to elicit significant improvements in peak $\dot{V} \mathrm{O}_{2}$, it may be postulated that the study used an inappropriate testing modality to assess adaptations in the dynamic $\dot{V} \mathrm{O}_{2}$ response. Whilst there is an associated age-related 
decline in the $\dot{V} \mathrm{O}_{2}$ kinetic response (Breese et al., 2010), the intervention was expected to have administered adaptations. Further work is warranted to conclude if exercise modality is important for differing exercise interventions which do not focus on specifically upper or lower body exercises. The use of a cross-trainer could have more closely assessed any adaptations which may have manifest in the whole-body HIIT intervention as the use of a cycle ergometer may not have had good transferability of training consisting of no cycling. Additionally or alternatively to confirm if the findings of the current thesis hold true that HIIT was able to offset the deleterious effects of maturation on the $\dot{V} \mathrm{O}_{2}$ kinetic response, the same HIIT protocol could be delivered on a cycle ergometer.

Research is required to determine the influence of asthma on the recovery $\dot{V} \mathrm{O}_{2}$ kinetic response from exercise. Recovery kinetics more closely reflect muscle phosphocreatine (Barker et al., 2008), a marker of oxidative capacity. As with the $\dot{V} \mathrm{O}_{2}$ kinetic response to exercise, the recovery kinetics are sensitive to adaptations due to the hemodynamic and cardiopulmonary changes that occur during the recovery phase (Bar et al., 2007). Whilst no differences were found between adolescents with and without asthma in the $\dot{V} \mathrm{O}_{2}$ kinetic response to exercise, differences could be manifest in the recovery stage with potential $\mathrm{O}_{2}$ delivery issues relating to the narrowing of airways and the aggravation of increased ventilation rate on asthma symptoms (Milgrom \& Taussig, 1999, Wanrooij et al., 2014). Indeed, $\dot{V} \mathrm{O}_{2}$ recovery kinetics may be shown to improve in adolescents with asthma following an exercise intervention as increases in cardiorespiratory fitness may lead to an improved ventilator equivalent for a given exercise intensity below maximal exercise.

Future studies should implement HIIT to maintain the levels of BMI in the adolescent populations of both those with and without asthma and consider the longer-term benefits of increased fitness on quality of life and asthma control. Physical activity levels should also be monitored to find if the increase in time spent in vigorous physical activity in the HIIT sessions alters the patterns of adolescents' overall activity; specifically if they have a compensatory reduction in activity outside of sessions (Ridgers et al., 2018a). As the current thesis struggled with participant wear time, the use of another previously validated more appealing monitor should be 
used to aid in adherence (Ridgers et al., 2018b). Furthermore, future studies should aim to implement inclusive HIIT interventions with greater numbers to maximise the possibility of uncovering significant effects on asthma control and quality of life.

In addition to formative research, future studies should consider pilot interventions to increase adherence. Furthermore, interventions that can be based around the school curriculum may be the way forward, increasing participation, as study retention for adolescents before and after school has been shown to result in a large dropout due to other commitments. Additionally, an intervention which is curriculum based and within school hours would also be able to target those adolescents who originally did not sign up to the study. Working with physical education staff to ensure future interventions fulfilled curriculum requirements could enhance physical education lessons and may also reduce the burden of teaching somewhat possibly increasing school recruitment.

One potential reason for the lack of difference in fitness between adolescents with and without asthma, may be the use of medication to control their asthma, effectively "normalising" their condition. Future research could establish the fitness differences between newly diagnosed adolescents with asthma, or those who do not take their prescribed medication. Furthermore, future research should aim to establish a more robust method to evaluate severity of asthma, instead of relying on medication step. Advances in severity evaluation will aid in the assessment of asthma throughout an exercise intervention. Finally, further research is also required for the novel phenotypes of asthma that have been recognised, such as the exercise asthma and obesity asthma phenotypes; in addition to a potential phenotype relating to a lack of fitness or physical activity. Moreover, if said phenotype exists, is it able to be modified, and if so, can this be done by influencing one parameter, be it BMI, fitness or physical activity? Additionally or alternatively, would all factors require altering for any modification to be manifest? 


\subsection{Conclusion}

In conclusion, work within this thesis has shown that although there was a general perception that adolescents with asthma are limited with regard to exercise, this is not the case as asthma, albeit mostly mild in the study, does not inhibit fitness in adolescents. Additionally, although adolescents with asthma fear asthma attacks caused by exercise, participating in exercise is still one of their favourite activities, showing promise for future interventions aimed at improving outcomes for those with asthma. Adolescents without asthma out-performed asthma participants in the field-based estimate of fitness, although this may have been related predominately to peer-influence based on perceptions. Indeed, when evaluating laboratory-derived fitness there were no differences in the four parameters of cardiorespiratory fitness between adolescents with and without asthma. Irrespective of condition, cardiorespiratory fitness was weakly correlated with quality of life and therefore improving fitness may aid in the management of asthma through a nonpharmacological treatment. The HIIT intervention implemented was able to maintain BMI and elicit significant improvements in peak $\dot{V} \mathrm{O}_{2}$ irrespective of asthma, suggesting the condition does not influence trainability. However, $\dot{V} \mathrm{O}_{2}$ kinetic responses remained unchanged throughout the intervention, possibly reflecting an attenuation of the typical age-related decline or due to modality differences. As there were no asthma attacks during the intervention, HIIT may be considered safe in adolescents with mild, well-controlled asthma.

Although, the HIIT intervention elicited no improvements in asthma-related quality of life and control, it may be suggested that an improvement in fitness could result in improvements over a prolonged period of time. Finally, if the results and future recommendations from this thesis are taken forward, education can be implemented into the benefits of exercise for adolescents with asthma. This may encourage adolescents to engage in life-long activity resulting in adults with a lower BMI, higher fitness and potentially better controlled asthma and a subsequent improved quality of life. Indeed according to the present thesis, HIIT represents an affordable, accessible, non-invasive and enjoyable form of asthma management. 
REFERENCE LIST 


\section{$\underline{\text { Reference list }}$}

AHMAIDI, S., VARRAY, A., SAVY-PACAUX, A. \& PREFAUT, C. 1993. Cardiorespiratory fitness evaluation by the shuttle test in asthmatic subjects during aerobic training. Chest, 103, 1135-41.

AKINBAMI, L. J., MOORMAN, J. E., BAILEY, C., ZAHRAN, H. S., KING, M., JOHNSON, C. A. \& LIU, X. 2012. Trends in asthma prevalence, health care use, and mortality in the United States, 2001-2010. NCHS Data Brief, 1-8.

ALBRECHT, G. H., GELVIN, B. R. \& HARTMAN, S. E. 1993. Ratios as a size adjustment in morphometrics. Am J Phys Anthropol, 91, 441-68.

AMERICAN THORACIC SOCIETY 1995. Standardization of Spirometry, 1994 Update. Am J Respir Crit Care Med, 152, 1107-36.

ANDERSEN, J. R., NATVIG, G. K., AADLAND, E., MOE, V. F., KOLOTKIN, R. L., ANDERSSEN, S. A. \& RESALAND, G. K. 2017. Associations between health-related quality of life, cardiorespiratory fitness, muscle strength, physical activity and waist circumference in 10-year-old children: the ASK study. Qual Life Res.

ANDERSON, G. P. 2008. Endotyping asthma: new insights into key pathogenic mechanisms in a complex, heterogeneous disease. Lancet, 372, 1107-19.

ANDERSON, L., OLDRIDGE, N., THOMPSON, D. R., ZWISLER, A. D., REES, K., MARTIN, N. \& TAYLOR, R. S. 2016. Exercise-Based Cardiac Rehabilitation for Coronary Heart Disease: Cochrane Systematic Review and Meta-Analysis. J Am Coll Cardiol, 67, 1-12.

ANDRADE, L. B., BRITTO, M. C., LUCENA-SILVA, N., GOMES, R. G. \& FIGUEROA, J. N. 2014. The efficacy of aerobic training in improving the inflammatory component of asthmatic children. Randomized trial. Respir Med, 108, 1438-45.

ANTHRACOPOULOS, M. B., FOUZAS, S., PAPADOPOULOS, M., ANTONOGEORGOS, G., PAPADIMITRIOU, A., PANAGIOTAKOS, D. B., NICOLAIDOU, P. \& PRIFTIS, K. N. 2012. Physical activity and exercise-induced bronchoconstriction in Greek schoolchildren. Pediatr Pulmonol, 47, 1080-7.

ARMSTONG, N. \& MCNARRY, M. 2016. Aerobic Fitness and Trainability in Healthy Youth: Gaps in Our Knowledge. Pediatr Exerc Sci, 28, 171-7.

ARMSTRONG, N. 2007. Paediatric exercise physiology. In: SPURWAY, S. \& MAXCLAREN, D. (eds.) Advances in sport and exercise science series. London, UK: Churchill Livingstone Elsevier.

ARMSTRONG, N. \& BARKER, A. R. 2009. Oxygen uptake kinetics in children and adolescents: a review. Pediatr Exerc Sci, 21, 130-47.

ARMSTRONG, N. \& BARKER, A. R. 2011. Endurance training and elite young athletes. Med Sport Sci, 56, 59-83.

ARMSTRONG, N., WELSMAN, J. \& WINSLEY, R. 1996. Is peak VO2 a maximal index of children's aerobic fitness? Int J Sports Med, 17, 356-9.

ARMSTRONG, N. \& WELSMAN, J. R. 1994. Assessment and interpretation of aerobic fitness in children and adolescents. Exerc Sport Sci Rev, 22, 435-76.

ASTHMA UK 2017. Asthma facts and statistics | Asthma UK.

BACHARIER, L. B., BONER, A., CARLSEN, K. H., EIGENMANN, P. A., FRISCHER, T., GOTZ, M., HELMS, P. J., HUNT, J., LIU, A., 
PAPADOPOULOS, N., PLATTS-MILLS, T., POHUNEK, P., SIMONS, F. E., VALOVIRTA, E., WAHN, U. \& WILDHABER, J. 2008. Diagnosis and treatment of asthma in childhood: a PRACTALL consensus report. Allergy, 63, 5-34.

BAEK, H. S., KIM, Y. D., SHIN, J. H., KIM, J. H., OH, J. W. \& LEE, H. B. 2011. Serum leptin and adiponectin levels correlate with exercise-induced bronchoconstriction in children with asthma. Ann Allergy Asthma Immunol, $107,14-21$.

BAILEY, S. J., WILKERSON, D. P., DIMENNA, F. J. \& JONES, A. M. 2009. Influence of repeated sprint training on pulmonary $\mathrm{O} 2$ uptake and muscle deoxygenation kinetics in humans. J Appl Physiol (1985), 106, 1875-87.

BAKER, D. 2011. Recent trends in high-intensity aerobic training for field sports. Prof Strength Cond, 4-8.

BALL, K., CRAWFORD, D. \& OWEN, N. 2000. Too fat to exercise? Obesity as a barrier to physical activity. Aust N Z J Public Health, 24, 331-3.

BAQUET, G., BERTHOIN, S., GERBEAUX, M. \& VAN PRAAGH, E. 2001. Highintensity aerobic training during a 10 week one-hour physical education cycle: effects on physical fitness of adolescents aged 11 to 16. Int J Sports Med, 22, 295-300.

BAQUET, G., VAN PRAAGH, E. \& BERTHOIN, S. 2003. Endurance training and aerobic fitness in young people. Sports Med, 33, 1127-43.

BARKER, A. R., WILLIAMS, C. A., JONES, A. M. \& ARMSTRONG, N. 2011. Establishing maximal oxygen uptake in young people during a ramp cycle test to exhaustion. British Journal of Sports Medicine, 45, 498-503.

BARLOW, S. E. 2007. Expert committee recommendations regarding the prevention, assessment, and treatment of child and adolescent overweight and obesity: summary report. Pediatrics, 120 Suppl 4, S164-92.

BARRON, A., FRANCIS, D. P., MAYET, J., EWERT, R., OBST, A., MASON, M., ELKIN, S., HUGHES, A. D. \& WENSEL, R. 2016. Oxygen Uptake Efficiency Slope and Breathing Reserve, Not Anaerobic Threshold, Discriminate Between Patients With Cardiovascular Disease Over Chronic Obstructive Pulmonary Disease. JACC Heart Fail, 4, 252-61.

BARROS, L. L., SOUZA-MACHADO, A., CORREA, L. B., SANTOS, J. S., CRUZ, C., LEITE, M., CASTRO, L., COELHO, A. C., ALMEIDA, P. \& CRUZ, A. A. 2011. Obesity and poor asthma control in patients with severe asthma. J Asthma, 48, 171-6.

BARSTOW, T. J., JONES, A. M., NGUYEN, P. H. \& CASABURI, R. 2000a. Influence of muscle fibre type and fitness on the oxygen uptake/power output slope during incremental exercise in humans. Exp Physiol, 85, 109-16.

BARSTOW, T. J. \& MOLE, P. A. 1991. Linear and nonlinear characteristics of oxygen uptake kinetics during heavy exercise. J Appl Physiol (1985), 71, 2099-106.

BARSTOW, T. J., SCREMIN, A. M., MUTTON, D. L., KUNKEL, C. F., CAGLE, T. G. \& WHIPP, B. J. 2000b. Peak and kinetic cardiorespiratory responses during arm and leg exercise in patients with spinal cord injury. Spinal Cord, $38,340-5$.

BASARAN, S., GULER-UYSAL, F., ERGEN, N., SEYDAOGLU, G., BINGOLKARAKOÇ, G. \& ALTINTAS, D. U. 2006. Effects of physical exercise on 
quality of life, exercise capacity and pulmonary function in children with asthma. Journal of Rehabilitation Medicine, 38, 130-135.

BEAUCHAMP, M. K., NONOYAMA, M., GOLDSTEIN, R. S., HILL, K., DOLMAGE, T. E., MATHUR, S. \& BROOKS, D. 2010. Interval versus continuous training in individuals with chronic obstructive pulmonary disease--a systematic review. Thorax, 65, 157-64.

BEAVER, W. L., WASSERMAN, K. \& WHIPP, B. J. 1986. A new method for detecting anaerobic threshold by gas exchange. J Appl Physiol (1985), 60, 2020-7.

BECKETT, W. S., JACOBS, D. R., JR., YU, X., IRIBARREN, C. \& WILLIAMS, O. D. 2001. Asthma is associated with weight gain in females but not males, independent of physical activity. Am J Respir Crit Care Med, 164, 2045-50.

BELAMARICH, P. F., LUDER, E., KATTAN, M., MITCHELL, H., ISLAM, S., LYNN, H. \& CRAIN, E. F. 2000. Do obese inner-city children with asthma have more symptoms than nonobese children with asthma? Pediatrics, 106, 1436-41.

BELCHER, B. R., MOSER, R. P., DODD, K. W., ATIENZA, A. A., BALLARDBARBASH, R. \& BERRIGAN, D. 2015. Self-Reported Versus Accelerometer-Measured Physical Activity and Biomarkers Among NHANES Youth. J Phys Act Health, 12, 708-16.

BERNTSEN, S. 2011. Physical Activity in Childhood Asthma: Friend or Foe? American Journal of Lifestyle Medicine, 5, 33-39.

BERNTSEN, S., CARLSEN, K. C., ANDERSSEN, S. A., MOWINCKEL, P., HAGEBERG, R., BUESO, A. K. \& CARLSEN, K. H. 2009. Norwegian adolescents with asthma are physical active and fit. Allergy, 64, 421-6.

BERNTSEN, S., DEPARTMENT OF PAEDIATRICS, O. U. H., OSLO, NORWAY \& DEPARTMENT OF SPORTS MEDICINE, N. S. O. S. S., OSLO, NORWAY 2016. Physical activity and aerobic fitness in childhood: associated with asthma? The Clinical Respiratory Journal, 5, 59-59.

BEUTHER, D. A., WEISS, S. T. \& SUTHERLAND, E. R. 2006. Obesity and asthma. American journal of respiratory and critical care medicine, 174, 112--9.

BLACK, M. H., SMITH, N., PORTER, A. H., JACOBSEN, S. J. \& KOEBNICK, C. 2012. Higher prevalence of obesity among children with asthma. Obesity (Silver Spring), 20, 1041-7.

BLAIR, S. N., KOHL, H. W., 3RD, PAFFENBARGER, R. S., JR., CLARK, D. G., COOPER, K. H. \& GIBBONS, L. W. 1989. Physical fitness and all-cause mortality. A prospective study of healthy men and women. Jama, 262, 2395401.

BODDY, L. M., THOMAS, N. E., FAIRCLOUGH, S. J., TOLFREY, K., BROPHY, S., REES, A., KNOX, G., BAKER, J. S. \& STRATTON, G. 2012. ROC Generated Thresholds for Field-Assessed Aerobic Fitness Related to Body Size and Cardiometabolic Risk in Schoolchildren. PLOS ONE, 7, e45755.

BONGERS, B. C., WERKMAN, M. S., TAKKEN, T. \& HULZEBOS, E. H. 2014. Ventilatory response to exercise in adolescents with cystic fibrosis and mildto-moderate airway obstruction. Springerplus, 3, 696.

BOONE, J. \& BOURGOIS, J. 2012. The oxygen uptake response to incremental ramp exercise: methodogical and physiological issues. Sports Med, 42, 51126. 
BOONE, J., KOPPO, K. \& BOUCKAERT, J. 2008. The VO2 response to submaximal ramp cycle exercise: Influence of ramp slope and training status. Respir Physiol Neurobiol, 161, 291-7.

BOUCHARD, C., BLAIR, S. N. \& HASKELL, W. L. 2012. Why study physical activity and health? In: BOUCHARD, C., BLAIR, S. N. \& HASKELL, W. L. (eds.) Physical activity and health second edition. Human Kinetics.

BOULET, L. P. \& FRANSSEN, E. 2007. Influence of obesity on response to fluticasone with or without salmeterol in moderate asthma. Respir Med, 101, 2240-7.

BOUSHEL, R., LANGBERG, H., OLESEN, J., GONZALES-ALONZO, J., BULOW, J. \& KJAER, M. 2001. Monitoring tissue oxygen availability with near infrared spectroscopy (NIRS) in health and disease. Scand J Med Sci Sports, 11, 213-22.

BOUSQUET, J., ANTO, J., AUFFRAY, C., AKDIS, M., CAMBON-THOMSEN, A., KEIL, T., HAAHTELA, T., LAMBRECHT, B. N., POSTMA, D. S., SUNYER, J., VALENTA, R., AKDIS, C. A., ANNESI-MAESANO, I., ARNO, A., BACHERT, C., BALLESTER, F., BASAGANA, X., BAUMGARTNER, U., BINDSLEV-JENSEN, C., BRUNEKREEF, B., CARLSEN, K. H., CHATZI, L., CRAMERI, R., EVENO, E., FORASTIERE, F., GARCIA-AYMERICH, J., GUERRA, S., HAMMAD, H., HEINRICH, J., HIRSCH, D., JACQUEMIN, B., KAUFFMANN, F., KERKHOF, M., KOGEVINAS, M., KOPPELMAN, G. H., KOWALSKI, M. L., LAU, S., LODRUP-CARLSEN, K. C., LOPEZ-BOTET, M., LOTVALL, J., LUPINEK, C., MAIER, D., MAKELA, M. J., MARTINEZ, F. D., MESTRES, J., MOMAS, I., NAWIJN, M. C., NEUBAUER, A., ODDIE, S., PALKONEN, S., PIN, I., PISON, C., RANCE, F., REITAMO, S., RIALSEBBAG, E., SALAPATAS, M., SIROUX, V., SMAGGHE, D., TORRENT, M., TOSKALA, E., VAN CAUWENBERGE, P., VAN OOSTERHOUT, A. J., VARRASO, R., VON HERTZEN, L., WICKMAN, M., WIJMENGA, C., WORM, M., WRIGHT, J. \& ZUBERBIER, T. 2011. MeDALL (Mechanisms of the Development of ALLergy): an integrated approach from phenotypes to systems medicine. Allergy, 66, 596-604.

BOYD, A., YANG, C. T., ESTELL, K., MS, C. T., GERALD, L. B., DRANSFIELD, M., BAMMAN, M., BONNER, J., ATKINSON, T. P. \& SCHWIEBERT, L. M. 2012. Feasibility of exercising adults with asthma: a randomized pilot study. Allergy Asthma Clin Immunol, 8, 13.

BRASIER, A. R., VICTOR, S., JU, H., BUSSE, W. W., CURRAN-EVERETT, D., BLEECKER, E., CASTRO, M., CHUNG, K. F., GASTON, B., ISRAEL, E., WENZEL, S. E., ERZURUM, S. C., JARJOUR, N. N. \& CALHOUN, W. J. 2010. Predicting intermediate phenotypes in asthma using bronchoalveolar lavage-derived cytokines. Clin Transl Sci, 3, 147-57.

BREESE, B. C., WILLIAMS, C. A., BARKER, A. R., WELSMAN, J. R., FAWKNER, S. G. \& ARMSTRONG, N. 2010. Longitudinal changes in the oxygen uptake kinetic response to heavy-intensity exercise in 14- to 16-yearold boys. Pediatr Exerc Sci, 22, 69-80.

BRITISH GUIDELINE ON THE MANAGEMENT OF ASTHMA 2008. British Guideline on the Management of Asthma. Thorax, 63 Suppl 4, iv1-121.

BRITISH THORACIC SOCIETY 2016. British guideline on the management of asthma. Available from: www.brit-thoracic.org.uk. 
BUCHAN, D. S., OLLIS, S., THOMAS, N. E., BUCHANAN, N., COOPER, S. M., MALINA, R. M. \& BAKER, J. S. 2011. Physical activity interventions: effects of duration and intensity. Scandinavian Journal of Medicine \& Science in Sports, 21, 341-50.

BUCHHEIT, M. \& LAURSEN, P. B. 2013. High-intensity interval training, solutions to the programming puzzle: Part I: cardiopulmonary emphasis. Sports Med, 43, 313-38.

CAIRNEY, J., HAY, J. A., FAUGHT, B. E., LEGER, L. \& MATHERS, B. 2008. Generalized self-efficacy and performance on the 20-metre shuttle run in children. Am J Hum Biol, 20, 132-8.

CALLERY, P., MILNES, L., VERDUYN, C. \& COURIEL, J. 2003. Qualitative study of young people's and parents' beliefs about childhood asthma. $\mathrm{Br} J$ Gen Pract, 53, 185-90.

CAMARGO, C. A., JR., WEISS, S. T., ZHANG, S., WILLETT, W. C. \& SPEIZER, F. E. 1999. Prospective study of body mass index, weight change, and risk of adult-onset asthma in women. Arch Intern Med, 159, 2582-8.

CAREY, D. G. \& RICHARDSON, M. T. 2003. Can Aerobic and Anaerobic Power be Measured in a 60-Second Maximal Test? J Sports Sci Med, 2, 151-7.

CARLSEN, K. H., HEM, E. \& STENSRUD, T. 2011. Asthma in adolescent athletes. British Journal of Sports Medicine, 45, 1266-1271.

CARSON, K. V., CHANDRATILlEKE, M. G., PICOT, J., BRINN, M. P., ESTERMAN, A. J. \& SMITH, B. J. 2013. Physical training for asthma. Cochrane Database Syst Rev, Cd001116.

CASPERSEN, C. J., POWELL, K. E. \& CHRISTENSON, G. M. 1985. Physical activity, exercise, and physical fitness: definitions and distinctions for healthrelated research. Public Health Rep, 100, 126-31.

CASSIM, R., KOPLIN, J. J., DHARMAGE, S. C., SENARATNA, B. C., LODGE, C. J., LOWE, A. J. \& RUSSELL, M. A. 2016. The difference in amount of physical activity performed by children with and without asthma: A systematic review and meta-analysis. J Asthma, 53, 882-92.

CATELLIER, D. J., HANNAN, P. J., MURRAY, D. M., ADDY, C. L., CONWAY, T. L., YANG, S. \& RICE, J. C. 2005. Imputation of missing data when measuring physical activity by accelerometry. Med Sci Sports Exerc, 37, S555-62.

CHAKIR, J., HAMID, Q., BOSSE, M., BOULET, L. P. \& LAVIOLETTE, M. 2002. Bronchial inflammation in corticosteroid-sensitive and corticosteroidresistant asthma at baseline and on oral corticosteroid treatment. Clin Exp Allergy, 32, 578-82.

CHANDRATILLEKE, M. G., CARSON, K. V., PICOT, J., BRINN, M. P., ESTERMAN, A. J. \& SMITH, B. J. 2012. Physical training for asthma. Cochrane Database Syst Rev, Cd001116.

CHANG, Y., YANG, Y., CHEN, C. \& CHIANG, B. 2008. Tai Chi Chuan training improves the pulmonary function of asthmatic children. Journal of microbiology, immunology, and infection, 41, 88-95.

CHAPMAN, S., DALE, P., SVEDSATER, H., STYNES, G., VYAS, N., PRICE, D. \& HORNE, R. 2017. Modelling the effect of beliefs about asthma medication and treatment intrusiveness on adherence and preference for once-daily vs. twice-daily medication. NPJ Prim Care Respir Med, 27, 61. 
CHEN, Y., DALES, R. \& KREWSKI, D. 2001. Leisure-time energy expenditure in asthmatics and non-asthmatics. Respir Med, 95, 13-8.

CHEN, Y. C., DONG, G. H., LIN, K. C. \& LEE, Y. L. 2013. Gender difference of childhood overweight and obesity in predicting the risk of incident asthma: a systematic review and meta-analysis. Obes Rev, 14, 222-31.

CHIANG, L. C., HUANG, J. L. \& FU, L. S. 2006. Physical activity and physical self-concept: comparison between children with and without asthma. $J$ Adv Nurs, 54, 653-62.

CHRISTENSEN, P. M., JACOBS, R. A., BONNE, T., FLUCK, D., BANGSBO, J. \& LUNDBY, C. 2016. A short period of high-intensity interval training improves skeletal muscle mitochondrial function and pulmonary oxygen uptake kinetics. J Appl Physiol (1985), 120, 1319-27.

CLARK, C. J. \& COCHRANE, L. M. 1988. Assessment of work performance in asthma for determination of cardiorespiratory fitness and training capacity. Thorax, 43, 745-9.

CleuZIOU, C., LECOQ, A. M., CANDAU, R., COURTEIX, D., GUENON, P. \& OBERT, P. 2002. Kinetics of oxygen uptake at the onset of moderate and heavy exercise in trained and untrained prepubertal children. Science \& Sports, 17, 291-296.

COCHRANE, L. \& CLARK, C. 1990. Benefits and problems of a physical training programme for asthmatic patients. Thorax, 45, 345-51.

COHEN, R., FRANCO, K., MOTLOW, F., REZNIK, M. \& OZUAH, P. O. 2003. Perceptions and Attitudes of Adolescents with Asthma. J Asthma, 40, 207211.

COQUART, J. B., LEMAIRE, C., DUBART, A. E., LUTTEMBACHER, D. P., DOUILLARD, C. \& GARCIN, M. 2008. Intermittent versus continuous exercise: effects of perceptually lower exercise in obese women. Med Sci Sports Exerc, 40, 1546-53.

CORDER, K., CRAGGS, C., JONES, A. P., EKELUND, U., GRIFFIN, S. J. \& VAN SLUIJS, E. M. 2013. Predictors of change differ for moderate and vigorous intensity physical activity and for weekdays and weekends: a longitudinal analysis. Int J Behav Nutr Phys Act, 10, 69.

CORDER, K., SHARP, S. J., ATKIN, A. J., ANDERSEN, L. B., CARDON, G., PAGE, A., DAVEY, R., GRONTVED, A., HALLAL, P. C., JANZ, K. F., KORDAS, K., KRIEMLER, S., PUDER, J. J., SARDINHA, L. B., EKELUND, U. \& VAN SLUIJS, E. M. 2016. Age-related patterns of vigorous-intensity physical activity in youth: The International Children's Accelerometry Database. Prev Med Rep, 4, 17-22.

COSTIGAN, S. A., EATHER, N., PlOTNIKOFF, R. C., Hillman, C. H. \& LUBANS, D. R. 2016. High-Intensity Interval Training for Cognitive and Mental Health in Adolescents. Med Sci Sports Exerc, 48, 1985-93.

COSTIGAN, S. A., EATHER, N., PlOTNIKOFF, R. C., TAAFFE, D. R. \& LUBANS, D. R. 2015. High-intensity interval training for improving healthrelated fitness in adolescents: a systematic review and meta-analysis. $\mathrm{Br} J$ Sports Med, 49, 1253-61.

COUNIL, F., VARRAY, A., MATECKI, S., BEUREY, A., MARCHAL, P., VOISIN, M. \& PRÉFAUT, C. 2003. Training of aerobic and anaerobic fitness in children with asthma. Journal of Pediatrics, 142, 179-184. 
COUTO, M., STANG, J., HORTA, L., STENSRUD, T., SEVERO, M., MOWINCKEL, P., SILVA, D., DELGADO, L., MOREIRA, A. \& CARLSEN, K. H. 2015. Two distinct phenotypes of asthma in elite athletes identified by latent class analysis. J Asthma, 52, 897-904.

CRIMI, E., BARTALUCCI, C. \& BRUSASCO, V. 1996. Asthma, exercise and the immune system. Exerc Immunol Rev, 2, 45-64.

CRONBACH, L. J. 1951. Coefficient alpha and the internal structure of tests. Psychometrika, 16, 297-334.

CROSBIE, A. 2012. The Effect of Physical Training in Children With Asthma on Pulmonary Function, Aerobic Capacity and Health-Related Quality of Life: A Systematic Review of Randomized Control Trials. Pediatric Exercise Science, 24, 472-489.

CUNHA, G., LORENZI, T., SAPATA, K., LOPES, A. L., GAYA, A. C. \& OLIVEIRA, A. 2011. Effect of biological maturation on maximal oxygen uptake and ventilatory thresholds in soccer players: an allometric approach. $J$ Sports Sci, 29, 1029-39.

CUNHA, G. S., VAZ, M. A., GEREMIA, J. M., LEITES, G. T., BAPTISTA, R. R., LOPES, A. L. \& REISCHAK-OLIVEIRA, A. 2016. Maturity Status Does Not Exert Effects on Aerobic Fitness in Soccer Players After Appropriate Normalization for Body Size. Pediatr Exerc Sci, 28, 456-65.

DANTAS, F. M., CORREIA, M. A., JR., SILVA, A. R., PEIXOTO, D. M., SARINHO, E. S. \& RIZZO, J. A. 2014. Mothers impose physical activity restrictions on their asthmatic children and adolescents: an analytical crosssectional study. BMC Public Health, 14, 287.

DE SOUZA, M. C., EISENMANN, J. C., E SANTOS, D. V., DE CHAVES, R. N., DE MORAES FORJAZ, C. L. \& MAIA, J. A. 2015. Modeling the dynamics of BMI changes during adolescence. The Oporto Growth, Health and Performance Study. Int J Obes (Lond), 39, 1063-9.

DEL GIACCO, S. R., CARLSEN, K. H. \& DU TOIT, G. 2012. Allergy and sports in children. Pediatr Allergy Immunol, 23, 11-20.

DEL GIACCO, S. R., FIRINU, D., BJERMER, L. \& CARLSEN, K. H. 2015. Exercise and asthma: an overview. Eur Clin Respir J, 2, 27984.

DELGADO, J., BARRANCO, P. \& QUIRCE, S. 2008. Obesity and asthma. Journal of investigational allergology \& clinical immunology, 18, 420-5.

DELOREY, D. S., KOWALCHUK, J. M., HEENAN, A. P., DUMANOIR, G. R. \& PATERSON, D. H. 2007. Prior exercise speeds pulmonary O2 uptake kinetics by increases in both local muscle $\mathrm{O} 2$ availability and $\mathrm{O} 2$ utilization. $J$ Appl Physiol (1985), 103, 771-8.

DEPARTMENT OF HEALTH 2011. Start active, stay active: a report on physical activity from the four home countries. In: OFFICER, C. M. (ed.). London, UK.

DiXON, A. E., HOLGUIN, F., SOOD, A., SAlOME, C. M., PRATlEY, R. E., BEUTHER, D. A., CELEDON, J. C. \& SHORE, S. A. 2010. An official American Thoracic Society Workshop report: obesity and asthma. Proc Am Thorac Soc, 7, 325-35.

DIXON, J. B., CHAPMAN, L. \& O'BRIEN, P. 1999. Marked improvement in asthma after Lap-Band surgery for morbid obesity. Obes Surg, 9, 385-9.

DOGRA, S., KUK, J. L., BAKER, J. \& JAMNIK, V. 2011. Exercise is associated with improved asthma control in adults. Eur Respir J, 37, 318-23. 
DONAHUE, K. E., MIELENZ, T. J., SLOANE, P. D., CALlAHAN, L. F. \& DEVELLIS, R. F. 2006. Identifying supports and barriers to physical activity in patients at risk for diabetes. Prev Chronic Dis, 3, A119.

DONNELly, J. E., HILlMAN, C. H., CASTELli, D., ETNIER, J. L., LEE, S., TOMPOROWSKI, P., LAMBOURNE, K. \& SZABO-REED, A. N. 2016. Physical Activity, Fitness, Cognitive Function, and Academic Achievement in Children: A Systematic Review. Med Sci Sports Exerc, 48, 1197-222.

DOZ, M., CHOUAID, C., COM-RUELLE, L., CALVO, E., BROSA, M., ROBERT, J., DECUYPERE, L., PRIBIL, C., HUERTA, A. \& DETOURNAY, B. 2013. The association between asthma control, health care costs, and quality of life in France and Spain. BMC Pulm Med, 13, 15.

DUMITH, S. C., GIGANTE, D. P., DOMINGUES, M. R. \& KOHL, H. W., 3RD 2011. Physical activity change during adolescence: a systematic review and a pooled analysis. Int J Epidemiol, 40, 685-98.

DWEIK, R. A., BOGGS, P. B., ERZURUM, S. C., IRVIN, C. G., LEIGH, M. W., LUNDBERG, J. O., OLIN, A. C., PLUMMER, A. L. \& TAYLOR, D. R. 2011. An official ATS clinical practice guideline: interpretation of exhaled nitric oxide levels (FENO) for clinical applications. Am J Respir Crit Care Med, 184, 602-15.

DWYER, J. J., ALLISON, K. R., GOLDENBERG, E. R., FEIN, A. J., YOSHIDA, K. K. \& BOUTILIER, M. A. 2006. Adolescent girls' perceived barriers to participation in physical activity. Adolescence, 41, 75-89.

EDDOLLS, W. T. B., MCNARRY, M. A., STRATTON, G., WINN, C. O. N. \& MACKINTOSH, K. A. 2017. High-Intensity Interval Training Interventions in Children and Adolescents: A Systematic Review. Sports Med.

EICHENBERGER, P. A., DIENER, S. N., KOFMEHL, R. \& SPENGLER, C. M. 2013. Effects of exercise training on airway hyperreactivity in asthma: a systematic review and meta-analysis. Sports medicine (Auckland, N.Z.), 43, 1157--70.

EIJKEMANS, M., MOMMERS, M., DE VRIES, S. I., VAN BUUREN, S., STAFLEU, A., BAKKER, I. \& THIJS, C. 2008. Asthmatic symptoms, physical activity, and overweight in young children: a cohort study. Pediatrics, 121, e666-72.

EIJKEMANS, M., MOMMERS, M., DRAAISMA, J. M., THIJS, C. \& PRINS, M. H. 2012. Physical activity and asthma: a systematic review and meta-analysis. PLoS One. United States.

ELIAKIM, A., BARSTOW, T. J., BRASEL, J. A., AJIE, H., LEE, W. N., RENSLO, R., BERMAN, N. \& COOPER, D. M. 1996. Effect of exercise training on energy expenditure, muscle volume, and maximal oxygen uptake in female adolescents. J Pediatr, 129, 537-43.

EMTNER, M. 1996. High-Intensity Physical Training in Adults with Asthma. CHEST Journal, 109, 323.

EMTNER, M., FINNE, M. \& STALENHEIM, G. 1998. High-intensity physical training in adults with asthma. A comparison between training on land and in water. Scand J Rehabil Med, 30, 201-9.

EUROPEAN ASSOCIATION FOR THE STUDY OF OBESITY 2018. Obesity facts and figures. Available from: www.easo.org. 
EVENSON, K. R., CATELLIER, D. J., GILL, K., ONDRAK, K. S. \& MCMURRAY, R. G. 2008. Calibration of two objective measures of physical activity for children. $J$ Sports $S c i, 26,1557-65$.

FALK, B. \& DOTAN, R. 2006. Child-adult differences in the recovery from highintensity exercise. Exerc Sport Sci Rev, 34, 107-12.

FANELlI, A., CABRAL, A. L. B., NEDER, J. A., MARTINS, M. A. \& CARVALHO, C. R. F. 2007. Exercise training on disease control and quality of life in asthmatic children. Medicine \& Science in Sports \& Exercise, 39, 1474-1480.

FARAH, C. S. \& SALOME, C. M. 2012. Asthma and obesity: a known association but unknown mechanism. Respirology, 17, 412-21.

FARINA, N., RUSTED, J. \& TABET, N. 2014. The effect of exercise interventions on cognitive outcome in Alzheimer's disease: a systematic review. Int Psychogeriatr, 26, 9-18.

FAWKNER, S. \& ARMSTRONG, N. 2003. Oxygen uptake kinetic response to exercise in children. Sports Med, 33, 651-69.

FAWKNER, S. G. \& ARMSTRONG, N. 2004a. Longitudinal changes in the kinetic response to heavy-intensity exercise in children. J Appl Physiol (1985), 97, 460-6.

FAWKNER, S. G. \& ARMSTRONG, N. 2004b. Sex differences in the oxygen uptake kinetic response to heavy-intensity exercise in prepubertal children. Eur J Appl Physiol, 93, 210-6.

FEDELE, D. A., JANICKE, D. M., LIM, C. S. \& ABU-HASAN, M. 2014. An examination of comorbid asthma and obesity: assessing differences in physical activity, sleep duration, health-related quality of life and parental distress. $J$ Asthma, 51, 275-81.

FEREDAY, J., MACDOUGALL, C., SPIZZO, M., DARBYSHIRE, P. \& SCHILLER, W. 2009. "There's nothing I can't do - I just put my mind to anything and I can do it": a qualitative analysis of how children with chronic disease and their parents account for and manage physical activity. BMC Pediatrics, 9, 1.

FIELDING, J., BRANTLEY, L., SEIGLER, N., MCKIE, K. T., DAVISON, G. W. \& HARRIS, R. A. 2015. Oxygen uptake kinetics and exercise capacity in children with cystic fibrosis. Pediatr Pulmonol, 50, 647-54.

FITCH, K. D., BLITVICH, J. D. \& MORTON, A. R. 1986. The effect of running training on exercise-induced asthma. Ann Allergy, 57, 90-4.

FLAPPER, B., DUIVERMAN, E., GERRITSEN, J., POSTEMA, K. \& SCHANS, C. 2008. Happiness to be gained in paediatric asthma care. The European respiratory journal, 32, 1555-62.

FORD, E. S. 2002. Does exercise reduce inflammation? Physical activity and Creactive protein among U.S. adults. Epidemiology, 13, 561-8.

FORD, E. S. 2005. The epidemiology of obesity and asthma. J Allergy Clin Immunol, 115, 897-909; quiz 910.

FORNO, E., LESCHER, R., STRUNK, R., WEISS, S., FUHLBRIGGE, A. \& CELEDON, J. C. 2011. Decreased response to inhaled steroids in overweight and obese asthmatic children. J Allergy Clin Immunol, 127, 741-9.

FORTE, G. C., GRUTCKI, D. M., MENEGOTTO, S. M., PEREIRA, R. P. \& DALCIN PDE, T. 2013. Prevalence of obesity in asthma and its relations with asthma severity and control. Rev Assoc Med Bras (1992), 59, 594-9. 
FOXDAL, P., SJODIN, A. \& SJODIN, B. 1996. Comparison of blood lactate concentrations obtained during incremental and constant intensity exercise. Int J Sports Med, 17, 360-5.

FRANKS, P. W., HANSON, R. L., KNOWLER, W. C., SIEVERS, M. L., BENNETT, P. H. \& LOOKER, H. C. 2010. Childhood obesity, other cardiovascular risk factors, and premature death. $N$ Engl J Med, 362, 485-93.

FUHLBRIGGE, A. L., GUILBERT, T., SPAHN, J., PEDEN, D. \& DAVIS, K. 2006. The influence of variation in type and pattern of symptoms on assessment in pediatric asthma. Pediatrics. United States.

GARFINKEL, S. K., KESTEN, S., CHAPMAN, K. R. \& REBUCK, A. S. 1992. Physiologic and nonphysiologic determinants of aerobic fitness in mild to moderate asthma. Am Rev Respir Dis, 145, 741-5.

GHABASHI, A. E. \& IQBAL, M. 2006. Obesity and its correlation with spirometric variables in patients with asthma. MedGenMed, 8, 58.

GIBALA, M. J., LITTLE, J. P., MACDONALD, M. J. \& HAWLEY, J. A. 2012. Physiological adaptations to low-volume, high-intensity interval training in health and disease. J Physiol, 590, 1077-84.

GIBALA, M. J. \& MCGEE, S. L. 2008. Metabolic adaptations to short-term highintensity interval training: a little pain for a lot of gain? Exerc Sport Sci Rev, 36, 58-63.

GIBSON, F. 2007. Conducting focus groups with children and young people: strategies for success. Journal of Reseach in Nursing, 12, 473-483.

GIMENES, A. C., NEDER, J. A., DAL CORSO, S., NOGUEIRA, C. R., NAPOLIS, L., MELlO, M. T., BULLE, A. S. \& NERY, L. E. 2011. Relationship between work rate and oxygen uptake in mitochondrial myopathy during ramp-incremental exercise. Braz J Med Biol Res, 44, 354-60.

GLANTZ, S. A. 1990. A method to estimate the point at which two lines intersect from noisy data. Am J Physiol, 259, H1305-6.

GLAZEBROOK, C., MCPHERSON, A. C., MACDONALD, I. A., SWIFT, J. A., RAMSAY, C., NEWBOULD, R. \& SMYTH, A. 2006. Asthma as a barrier to children's physical activity: implications for body mass index and mental health. Pediatrics, 118, 2443-2449.

GLOBAL INITIATIVE FOR ASTHMA 2017. Global strategy for asthma management and prevention. Available from: www.ginasthma.org.

GONCALVES, R. C., NUNES, M. P. T., CUKIER, A., STELMACH, R., MARTINS, M. A. \& CARVALHO, C. R. F. 2008. Effects of an aerobic physical training program on psychosocial characteristics, quality-of-life, symptoms and exhaled nitric oxide in individuals with moderate or severe persistent asthma. Revista Brasileira de Fisioterapia, 12, 127-135.

GOV 2017. Childhood obesity: a plan for action. Available from: www.gov.uk.

GRAFF-LONNEVIG, V., BEVEGARD, S. \& ERIKSSON, B. 1980. Two years' follow-up of asthmatic boys participating in a physical activity programme. Acta paediatrica Scandinavica, 69, 347-52.

GRASSI, B., POGLIAGHI, S., RAMPICHINI, S., QUARESIMA, V., FERRARI, M., MARCONI, C. \& CERRETELLI, P. 2003. Muscle oxygenation and pulmonary gas exchange kinetics during cycling exercise on-transitions in humans. J Appl Physiol (1985), 95, 149-58. 
GRASSI, B., POOLE, D. C., RICHARDSON, R. S., KNIGHT, D. R., ERICKSON, B. K. \& WAGNER, P. D. 1996. Muscle O2 uptake kinetics in humans: implications for metabolic control. J Appl Physiol (1985), 80, 988-98.

GROEN, W. G., HULZEBOS, H. J., HELDERS, P. J. \& TAKKEN, T. 2010. Oxygen uptake to work rate slope in children with a heart, lung or muscle disease. Int J Sports Med, 31, 202-6.

GRZELEWSKI, T. \& STELMACH, I. 2009. Exercise-induced bronchoconstriction in asthmatic children: a comparative systematic review of the available treatment options. Drugs, 69, 1533-53.

GUERRA, S., SHERRILl, D. L., BOBADILlA, A., MARTINEZ, F. D. \& BARBEE, R. A. 2002. The relation of body mass index to asthma, chronic bronchitis, and emphysema. Chest, 122, 1256-63.

GUIRAUD, T., NIGAM, A., GREMEAUX, V., MEYER, P., JUNEAU, M. \& BOSQUET, L. 2012. High-intensity interval training in cardiac rehabilitation. Sports Med, 42, 587-605.

GULDBERG-MOLLER, J., HANCOX, B., MIKKELSEN, D., HANSEN, H. S. \& RASMUSSEN, F. 2015. Physical fitness and amount of asthma and asthmalike symptoms from childhood to adulthood. Clin Respir J, 9, 314-21.

GUSTAFSSON, P. M., WATSON, L., DAVIS, K. J. \& RABE, K. F. 2006. Poor asthma control in children: evidence from epidemiological surveys and implications for clinical practice. Int J Clin Pract, 60, 321-34.

HAAS, F., PASIERSKI, S., LEVINE, N., BISHOP, M., AXEN, K., PINEDA, H. \& HAAS, A. 1987. Effect of aerobic training on forced expiratory airflow in exercising asthmatic humans. J Appl Physiol (1985), 63, 1230-5.

HALDAR, P., PAVORD, I. D., SHAW, D. E., BERRY, M. A., THOMAS, M., BRIGHTLING, C. E., WARDLAW, A. J. \& GREEN, R. H. 2008. Cluster analysis and clinical asthma phenotypes. Am J Respir Crit Care Med, 178, 218-224.

HALLAL, P. C., ANDERSEN, L. B., BULL, F. C., GUTHOLD, R., HASKELL, W. \& EKELUND, U. 2012. Global physical activity levels: surveillance progress, pitfalls, and prospects. Lancet, 380, 247-57.

HALLSTRAND, T. S., BATES, P. W. \& SCHOENE, R. B. 2000. Aerobic conditioning in mild asthma decreases the hyperpnea of exercise and improves exercise and ventilatory capacity. Chest, 118, 1460-9.

HANSEN, J. E., SUE, D. Y., OREN, A. \& WASSERMAN, K. 1987. Relation of oxygen uptake to work rate in normal men and men with circulatory disorders. Am J Cardiol, 59, 669-74.

HASKELL, W. L., LEE, I. M., PATE, R. R., POWELL, K. E., BLAIR, S. N., FRANKLIN, B. A., MACERA, C. A., HEATH, G. W., THOMPSON, P. D. \& BAUMAN, A. 2007. Physical activity and public health: updated recommendation for adults from the American College of Sports Medicine and the American Heart Association. Med Sci Sports Exerc, 39, 1423-34.

HAUGHNEY, J., BARNES, G., PARTRIDGE, M. \& CLELAND, J. 2004. The Living \& Breathing Study: a study of patients' views of asthma and its treatment. Prim Care Respir J, 13, 28-35.

HENNESSY, E. \& HEARY, C. 2005. Exploring children's views through focus groups. In: GREENE, S. \& HOGAN, D. M. (eds.) Researching children's experiences: Approaches and methods. London: Sage. 
HINGORJO, M. R., ZEHRA, S., HASAN, Z. \& QURESHI, M. A. 2017. Cardiorespiratory fitness and its association with adiposity indices in young adults. Pak J Med Sci, 33, 659-664.

HOLGATE, S. T. 2011. Pathophysiology of asthma: what has our current understanding taught us about new therapeutic approaches? J Allergy Clin Immunol, 128, 495-505.

HOLGUIN, F., BLEECKER, E. R., BUSSE, W. W., CALHOUN, W. J., CASTRO, M., ERZURUM, S. C., FITZPATRICK, A. M., GASTON, B., ISRAEL, E., JARJOUR, N. N., MOORE, W. C., PETERS, S. P., YONAS, M., TEAGUE, W. G. \& WENZEL, S. E. 2011. Obesity and asthma: an association modified by age of asthma onset. J Allergy Clin Immunol, 127, 1486-93.e2.

HOLZER, F. J., SCHNALL, R. \& LANDAU, L. I. 1984. The effect of a home exercise programme in children with cystic fibrosis and asthma. Aust Paediatr J, 20, 297-301.

HOOD, M. S., LITTLE, J. P., TARNOPOLSKY, M. A., MYSLIK, F. \& GIBALA, M. J. 2011. Low-volume interval training improves muscle oxidative capacity in sedentary adults. Med Sci Sports Exerc, 43, 1849-56.

HORNER, S. D. 2000. Using focus group methods with middle school children. Res Nurs Health, 23, 510-7.

HUANG, S. W., VEIGA, R., SILA, U., REED, E. \& HINES, S. 1989. The effect of swimming in asthmatic children--participants in a swimming program in the city of Baltimore. J Asthma, 26, 117-21.

HWANG, C. L., WU, Y. T. \& CHOU, C. H. 2011. Effect of aerobic interval training on exercise capacity and metabolic risk factors in people with cardiometabolic disorders: a meta-analysis. J Cardiopulm Rehabil Prev, 31, 378-85.

JANSSEN, I. \& LEBLANC, A. G. 2010. Systematic review of the health benefits of physical activity and fitness in school-aged children and youth. Int J Behav Nutr Phys Act, 7, 40.

JENSEN, M. E., COLliNS, C. E., GIBSON, P. G. \& WOOD, L. G. 2011. The obesity phenotype in children with asthma. Paediatr Respir Rev, 12, 152-9.

JENSEN, M. E., GIBSON, P. G., COLLINS, C. E., HILTON, J. M. \& WOOD, L. G. 2013. Diet-induced weight loss in obese children with asthma: a randomized controlled trial. Clin Exp Allergy, 43, 775-84.

JONES, A. M. 2006. The Physiology of the World Record Holder for the Women's Marathon. Int J Sports Sci Coa, 1, 101-116.

JONES, A. M. \& POOLE, D. C. 2005. Oxygen uptake dynamics: from muscle to mouth-an introduction to the symposium. Med Sci Sports Exerc, 37, 1542-50.

JONES, S., ELLIOTT, P. M., SHARMA, S., MCKENNA, W. J. \& WHIPP, B. J. 1998. Cardiopulmonary responses to exercise in patients with hypertrophic cardiomyopathy. Heart, 80, 60-7.

JONSSON, M., EGMAR, A. C., HALLNER, E. \& KULL, I. 2014. Experiences of living with asthma - a focus group study with adolescents and parents of children with asthma. J Asthma, 51, 185-92.

JUNIPER, E. F., GRUFFYDD-JONES, K., WARD, S. \& SVENSSON, K. 2010. Asthma Control Questionnaire in children: validation, measurement properties, interpretation. Eur Respir J, 36, 1410-6. 
JUNIPER, E. F., GUYATT, G. H., FEENY, D. H., FERRIE, P. J., GRIFFITH, L. E. \& TOWNSEND, M. 1996. Measuring quality of life in children with asthma. Qual Life Res, 5, 35-46.

KAPLAN, T. A. \& MONTANA, E. 1993. Exercise-induced bronchospasm in nonasthmatic obese children. Clin Pediatr (Phila), 32, 220-5.

KESSLER, H. S., SISSON, S. B. \& SHORT, K. R. 2012. The potential for highintensity interval training to reduce cardiometabolic disease risk. Sports Med, 42, 489-509.

KING, M. J., NOAKES, T. D. \& WEINBERG, E. G. 1989. Physiological Effects of a Physical Training Program in Children with Exercise-Induced Asthma. Pediatr Exerc Sci, 1, 137-144.

KITSANTAS, A. \& ZIMMERMAN, B. J. 2000. Self-efficacy, activity participation, and physical fitness of asthmatic and nonasthmatic adolescent girls. $J$ Asthma, 37, 163-74.

KODAMA, S., SAITO, K., TANAKA, S., MAKI, M., YACHI, Y., ASUMI, M., SUGAWARA, A., TOTSUKA, K., SHIMANO, H., OHASHI, Y., YAMADA, N. \& SONE, H. 2009. Cardiorespiratory fitness as a quantitative predictor of all-cause mortality and cardiovascular events in healthy men and women: a meta-analysis. Jama, 301, 2024-35.

KRIEMLER, S., MEYER, U., MARTIN, E., VAN SLUIJS, E., ANDERSEN, L. \& MARTIN, B. 2011. Effect of school-based interventions on physical activity and fitness in children and adolescents: A review of reviews and systematic update. Br J Sports Med, 45.

KRIKETOS, A. D., BAUR, L. A., O'CONNOR, J., CAREY, D., KING, S., CATERSON, I. D. \& STORLIEN, L. H. 1997. Muscle fibre type composition in infant and adult populations and relationships with obesity. Int J Obes Relat Metab Disord, 21, 796-801.

KRUSTRUP, P., HELLSTEN, Y. \& BANGSBO, J. 2004. Intense interval training enhances human skeletal muscle oxygen uptake in the initial phase of dynamic exercise at high but not at low intensities. $J$ Physiol, 559, 335-45.

KRUSTRUP, P., JONES, A. M., WILKERSON, D. P., CALBET, J. A. \& BANGSBO, J. 2009. Muscular and pulmonary O2 uptake kinetics during moderate- and high-intensity sub-maximal knee-extensor exercise in humans. J Physiol, 587, 1843-56.

LAI, N., MARTIS, A., BELFIORI, A., TOLENTINO-SILVA, F., NASCA, M. M., STRAINIC, J. \& CABRERA, M. E. 2016. Gender differences in V O2 and HR kinetics at the onset of moderate and heavy exercise intensity in adolescents. Physiol Rep, 4.

LAMBRICK, D., WESTRUPP, N., KAUFMANN, S., STONER, L. \& FAULKNER, J. 2016. The effectiveness of a high-intensity games intervention on improving indices of health in young children. J Sports Sci, 34, 190-8.

LANG, D. M., BUTZ, A. M., DUGGAN, A. K. \& SERWINT, J. R. 2004. Physical activity in urban school-aged children with asthma. Pediatrics, 113, 341-346.

LANG, J. E. 2014. Obesity and asthma in children: current and future therapeutic options. Paediatr Drugs, 16, 179-88.

LANG, J. E., HOSSAIN, J., DIXON, A. E., SHADE, D., WISE, R. A., PETERS, S. P. \& LIMA, J. J. 2011. Does age impact the obese asthma phenotype? Longitudinal asthma control, airway function, and airflow perception among mild persistent asthmatics. Chest, 140, 1524-1533. 
LATORRE-ROMAN, P. A., NAVARRO-MARTINEZ, A. V. \& GARCIAPINILLOS, F. 2014. The effectiveness of an indoor intermittent training program for improving lung function, physical capacity, body composition and quality of life in children with asthma. $J$ Asthma, 51, 544-51.

LAU, P. W., WONG DEL, P., NGO, J. K., LIANG, Y., KIM, C. G. \& KIM, H. S. 2015. Effects of high-intensity intermittent running exercise in overweight children. Eur J Sport Sci, 15, 182-90.

LAURSEN, P. B. \& JENKINS, D. G. 2002. The scientific basis for high-intensity interval training: optimising training programmes and maximising performance in highly trained endurance athletes. Sports Med, 32, 53-73.

LAVENEZIANA, P., LOTTI, P., COLI, C., BINAZZI, B., CHITI, L., STENDARDI, L., DURANTI, R. \& SCANO, G. 2006. Mechanisms of dyspnoea and its language in patients with asthma. Eur Respir J, 27, 742-747.

LAVOIE, K. L., BACON, S. L., LABRECQUE, M., CARTIER, A. \& DITTO, B. 2006. Higher BMI is associated with worse asthma control and quality of life but not asthma severity. Respir Med, 100, 648-57.

LAWSON, J. A., RENNIE, D. C., DOSMAN, J. A., CAMMER, A. L. \& SENTHILSELVAN, A. 2013. Obesity, diet, and activity in relation to asthma and wheeze among rural dwelling children and adolescents. J Obes, 2013, 315096.

LEINAAR, E., ALAMIAN, A. \& WANG, L. 2016. A systematic review of the relationship between asthma, overweight, and the effects of physical activity in youth. Annals of Epidemiology, 26, 504-510.e6.

LESSARD, A., TURCOTTE, H., CORMIER, Y. \& BOULET, L. P. 2008. Obesity and asthma: a specific phenotype? Chest, 134, 317-323.

LIMA, E. V., LIMA, W. L., NOBRE, A., DOS SANTOS, A. M., BRITO, L. M. \& COSTA MDO, R. 2008. Inspiratory muscle training and respiratory exercises in children with asthma. J Bras Pneumol, 34, 552-8.

LITTLE, J. P., GILLEN, J. B., PERCIVAL, M. E., SAFDAR, A., TARNOPOLSKY, M. A., PUNTHAKEE, Z., JUNG, M. E. \& GIBALA, M. J. 2011. Lowvolume high-intensity interval training reduces hyperglycemia and increases muscle mitochondrial capacity in patients with type 2 diabetes. $J$ Appl Physiol (1985), 111, 1554-60.

LOCHTE, L., ANGERMANN, M. \& LARSSON, B. 2009. Cardiorespiratory fitness of asthmatic children and validation of predicted aerobic capacity. Clin Respir J. England.

LOCHTE, L., NIELSEN, K. G., PETERSEN, P. E. \& PLATTS-MILLS, T. A. 2016. Childhood asthma and physical activity: a systematic review with metaanalysis and Graphic Appraisal Tool for Epidemiology assessment. BMC Pediatr, 16, 50.

LOGAN, G. R., HARRIS, N., DUNCAN, S. \& SCHOFIELD, G. 2014. A review of adolescent high-intensity interval training. Sports Med, 44, 1071-85.

LOPES, W. A., RADOMINSKI, R. B., ROSARIO FILHO, N. A. \& LEITE, N. 2009. Exercise-induced bronchospasm in obese adolescents. Allergol Immunopathol (Madr), 37, 175-9.

LOTVALL, J., AKDIS, C. A., BACHARIER, L. B., BJERMER, L., CASALE, T. B., CUSTOVIC, A., LEMANSKE, R. F., JR., WARDLAW, A. J., WENZEL, S. E. \& GREENBERGER, P. A. 2011. Asthma endotypes: a new approach to 
classification of disease entities within the asthma syndrome. J Allergy Clin Immunol, 127, 355-60.

LOVELL, G. P., EL ANSARI, W. \& PARKER, J. K. 2010. Perceived exercise benefits and barriers of non-exercising female university students in the United Kingdom. Int J Environ Res Public Health, 7, 784-98.

LU, K. D., MANOUKIAN, K., RADOM-AIZIK, S., COOPER, D. M. \& GALANT, S. P. 2016. Obesity, Asthma, and Exercise in Child and Adolescent Health. Pediatr Exerc Sci.

LUCAS, S. R. \& PLATTS-MILLS, T. A. 2005. Physical activity and exercise in asthma: relevance to etiology and treatment. J Allergy Clin Immunol, 115, 928-34.

LUCAS, S. R. \& PLATTS-MILLS, T. A. 2006. Paediatric asthma and obesity. Paediatr Respir Rev. England.

LUCIA, A., HOYOS, J., PEREZ, M., SANTALLA, A. \& CHICHARRO, J. L. 2002. Inverse relationship between VO2max and economy/efficiency in world-class cyclists. Med Sci Sports Exerc, 34, 2079-84.

LUPPINO, F. S., DE WIT, L. M., BOUVY, P. F., STIJNEN, T., CUIJPERS, P., PENNINX, B. W. \& ZITMAN, F. G. 2010. Overweight, obesity, and depression: a systematic review and meta-analysis of longitudinal studies. Arch Gen Psychiatry, 67, 220-9.

MACDONALD, M. J. \& CURRIE, K. D. 2009. Interval exercise is a path to good health, but how much, how often and for whom? Clin Sci (Lond), 116, 315-6.

MACKINTOSH, K. A., KNOWLES, Z. R., RIDGERS, N. D. \& FAIRCLOUGH, S. J. 2011. Using formative research to develop CHANGE! : a curriculum-based physical activity promoting intervention. BMC Public Health, 11, 831.

MACPHEE, S. L., SHOEMAKER, J. K., PATERSON, D. H. \& KOWALCHUK, J. M. 2005. Kinetics of $\mathrm{O} 2$ uptake, leg blood flow, and muscle deoxygenation are slowed in the upper compared with lower region of the moderate-intensity exercise domain. J Appl Physiol (1985), 99, 1822-34.

MAHON, A. D., MARJERRISON, A. D., LEE, J. D., WOODRUFF, M. E. \& HANNA, L. E. 2010. Evaluating the prediction of maximal heart rate in children and adolescents. Res Q Exerc Sport, 81, 466-71.

MALIK, A. A., WILLIAMS, C. A., BOND, B., WESTON, K. L. \& BARKER, A. R. 2017. Acute cardiorespiratory, perceptual and enjoyment responses to highintensity interval exercise in adolescents. European Journal of Sport Science, $1-8$.

MALINA, R. M. 2001. Physical activity and fitness: pathways from childhood to adulthood. Am J Hum Biol, 13, 162-72.

MANCUSO, C. A., CHOI, T. N., WESTERMANN, H., WENDEROTH, S., WELLS, M. T. \& CHARLSON, M. E. 2013. Improvement in asthma quality of life in patients enrolled in a prospective study to increase lifestyle physical activity. J Asthma, 50, 103-7.

MANDIGOUT, S., LECOQ, A. M., COURTEIX, D., GUENON, P. \& OBERT, P. 2001. Effect of gender in response to an aerobic training programme in prepubertal children. Acta Paediatr, 90, 9-15.

MANNINO, D. M., HOMA, D. M., AKINBAMI, L. J., MOORMAN, J. E., GWYNN, C. \& REDD, S. C. 2002. Surveillance for asthma--United States, 1980-1999. MMWR Surveill Summ, 51, 1-13. 
MARWOOD, S., ROCHE, D., ROWLAND, T., GARRARD, M. \& UNNITHAN, V. B. 2010. Faster pulmonary oxygen uptake kinetics in trained versus untrained male adolescents. Med Sci Sports Exerc, 42, 127-34.

MATOS, N. \& WINSLEY, R. J. 2007. Trainability of Young Athletes and Overtraining. J Sports Sci Med.

MATSUMOTO, I., ARAKI, H., TSUDA, K., ODAJIMA, H., NISHIMA, S., HIGAKI, Y., TANAKA, H., TANAKA, M. \& SHINDO, M. 1999. Effects of swimming training on aerobic capacity and exercise induced bronchoconstriction in children with bronchial asthma. Thorax, 54, 196-201.

MAYORGA-VEGA, D., AGUILAR-SOTO, P. \& VICIANA, J. 2015. CriterionRelated Validity of the 20-M Shuttle Run Test for Estimating Cardiorespiratory Fitness: A Meta-Analysis. J Sports Sci Med, 14, 536-47.

MCDONALD, A. S. 2001. The Prevalence and Effects of Test Anxiety in School Children. Educational Psychology, 21, 89-101.

MCKAY, B. R., PATERSON, D. H. \& KOWALCHUK, J. M. 2009. Effect of shortterm high-intensity interval training vs. continuous training on $\mathrm{O} 2$ uptake kinetics, muscle deoxygenation, and exercise performance. J Appl Physiol (1985), 107, 128-38.

MCMANUS, A. M., ARMSTRONG, N. \& WILLIAMS, C. A. 1997. Effect of training on the aerobic power and anaerobic performance of prepubertal girls. Acta Paediatr, 86, 456-9.

MCNARRY, M. \& ARMSTONG, N. 2017. Aerobic trainability. In: ARMSTRONG, N. \& VAN MECHELEN, W. (eds.) Oxford textbook of children's sport and exercise medicine Oxford, UK: Oxford University Pres.

MCNARRY, M. A., BODDY, L. M. \& STRATTON, G. S. 2014a. The relationship between body mass index, aerobic performance and asthma in a pre-pubertal, population-level cohort. Eur J Appl Physiol, 114, 243-9.

MCNARRY, M. A., FARR, C., MIDDLEBROOKE, A., WELFORD, D., BREESE, B., ARMSTRONG, N. \& BARKER, A. R. 2015a. Aerobic Function and Muscle Deoxygenation Dynamics during Ramp Exercise in Children. Med Sci Sports Exerc, 47, 1877-84.

MCNARRY, M. A., HARRISON, N. K., WITHERS, T., CHINNAPPA, N. \& LEWIS, M. J. 2017. Pulmonary oxygen uptake and muscle deoxygenation kinetics during heavy intensity cycling exercise in patients with emphysema and idiopathic pulmonary fibrosis. BMC Pulm Med, 17, 26.

MCNARRY, M. A., LAMBRICK, D., WESTRUPP, N. \& FAULKNER, J. 2015b. The influence of a six-week, high-intensity games intervention on the pulmonary oxygen uptake kinetics in prepubertal obese and normal-weight children. Appl Physiol Nutr Metab, 40, 1012-8.

MCNARRY, M. A., MACKINTOSH, K. A. \& STOEDEFALKE, K. 2014b. Longitudinal investigation of training status and cardiopulmonary responses in pre- and early-pubertal children. Eur J Appl Physiol, 114, 1573-80.

MCNARRY, M. A., WELSMAN, J. R. \& JONES, A. M. 2011a. The influence of training and maturity status on girls' responses to short-term, high-intensity upper- and lower-body exercise. Appl Physiol Nutr Metab, 36, 344-52.

MCNARRY, M. A., WELSMAN, J. R. \& JONES, A. M. 2011b. Influence of training and maturity status on the cardiopulmonary responses to ramp incremental cycle and upper body exercise in girls. J Appl Physiol (1985), $110,375-81$. 
MCNARRY, M. A., WELSMAN, J. R. \& JONES, A. M. 2011c. Influence of training status and exercise modality on pulmonary $\mathrm{O} 2$ uptake kinetics in pubertal girls. Eur J Appl Physiol, 111, 621-31.

MENDES, F. A., GONCALVES, R. C., NUNES, M. P., SARAIVAROMANHOLO, B. M., CUKIER, A., STELMACH, R., JACOB-FILHO, W., MARTINS, M. A. \& CARVALHO, C. R. 2010. Effects of aerobic training on psychosocial morbidity and symptoms in patients with asthma: a randomized clinical trial. Chest, 138, 331-7.

MERIKALLIO, V. J., MUSTALAHTI, K., REMES, S. T., VALOVIRTA, E. J. \& KAILA, M. 2005. Comparison of quality of life between asthmatic and healthy school children. Pediatr Allergy Immunol, 16, 332-40.

METCALF, B., HENLEY, W. \& WILKIN, T. 2012. Effectiveness of intervention on physical activity of children: systematic review and meta-analysis of controlled trials with objectively measured outcomes (EarlyBird 54). Bmj, 345, e5888.

MEYER, K., SCHWAIBOLD, M., HAJRIC, R., WESTBROOK, S., EBFELD, D., LEYK, D. \& ROSKAMM, H. 1998. Delayed VO2 kinetics during ramp exercise: a criterion for cardiopulmonary exercise capacity in chronic heart failure. Med Sci Sports Exerc, 30, 643-8.

MILANOVIC, Z., SPORIS, G. \& WESTON, M. 2015. Effectiveness of HighIntensity Interval Training (HIT) and Continuous Endurance Training for VO2max Improvements: A Systematic Review and Meta-Analysis of Controlled Trials. Sports Med, 45, 1469-81.

MILGROM, H. \& TAUSSIG, L. M. 1999. Keeping children with exercise-induced asthma active. Pediatrics, 104, e38.

MILLER, M. R., HANKINSON, J., BRUSASCO, V., BURGOS, F., CASABURI, R., COATES, A., CRAPO, R., ENRIGHT, P., VAN DER GRINTEN, C. P. M., GUSTAFSSON, P., JENSEN, R., JOHNSON, D. C., MACINTYRE, N., MCKAY, R., NAVAJAS, D., PEDERSEN, O. F., PELLEGRINO, R., VIEGI, G. \& WANGER, J. 2005. Standardisation of spirometry. European Respiratory Journal, 26, 319-338.

MINIC, P. B. \& SOVTIC, A. D. 2017. Exercise intolerance and exercise-induced bronchoconstriction in children. Front Biosci (Elite Ed), 9, 21-32.

MIOXHAM, J. \& JOLLEY, C. 2009. Breathlessness, fatigue and the respiratory muscles. Clin Med (Lond), 9, 448-52.

MIRWALD, R. L., BAXTER-JONES, A. D., BAILEY, D. A. \& BEUNEN, G. P. 2002. An assessment of maturity from anthropometric measurements. Medicine and Science in Sports and Exercise, 34, 689-694.

MIYAMOTO, Y., HIURA, T., TAMURA, T., NAKAMURA, T., HIGUCHI, J. \& MIKAMI, T. 1982. Dynamics of cardiac, respiratory, and metabolic function in men in response to step work load. J Appl Physiol Respir Environ Exerc Physiol, 52, 1198-208.

MOLZON, E. S., BONNER, M. S., HULLMANN, S. E., RAMSEY, R. R., SUORSA, K. I., CHANEY, J. M. \& MULLINS, L. L. 2013. Differences in sleep quality and health-related quality of life in young adults with allergies and asthma and their healthy peers. J Am Coll Health, 61, 484-9.

MONTOYE, H. J. 2000. Introduction: evaluation of some measurements of physical activity and energy expenditure. Med Sci Sports Exerc, 32, S439-41. 
MOREIRA, A., DELGADO, L., HAAHTELA, T., FONSECA, J., MOREIRA, P., LOPES, C., MOTA, J., SANTOS, P., RYTILA, P. \& CASTEL-BRANCO, M. G. 2008. Physical training does not increase allergic inflammation in asthmatic children. Eur Respir J, 32, 1570-5.

MUKHERJEE, M., STODDART, A., GUPTA, R. P., NWARU, B. I., FARR, A., HEAVEN, M., FITZSIMMONS, D., BANDYOPADHYAY, A., AFTAB, C., SIMPSON, C. R., LYONS, R. A., FISCHBACHER, C., DIBBEN, C., SHIELDS, M. D., PHILliPS, C. J., STRACHAN, D. P., DAVIES, G. A., MCKINSTRY, B. \& SHEIKH, A. 2016. The epidemiology, healthcare and societal burden and costs of asthma in the UK and its member nations: analyses of standalone and linked national databases. BMC Med, 14, 113.

MURIAS, J. M., EDWARDS, J. A. \& PATERSON, D. H. 2016. Effects of shortterm training and detraining on VO2 kinetics: Faster VO2 kinetics response after one training session. Scand J Med Sci Sports, 26, 620-9.

MURIAS, J. M., KOWALCHUK, J. M. \& PATERSON, D. H. 2010. Speeding of VO2 kinetics with endurance training in old and young men is associated with improved matching of local O2 delivery to muscle $\mathrm{O} 2$ utilization. $J$ Appl Physiol (1985), 108, 913-22.

MURIAS, J. M., SPENCER, M. D., KOWALCHUK, J. M. \& PATERSON, D. H. 2011. Muscle deoxygenation to VO2 relationship differs in young subjects with varying $\tau \mathrm{VO} 2$. European Journal of Applied Physiology, 111, 31073118.

MURPHY, A. C., PROESCHAl, A., BRIGHTling, C. E., WARDLAW, A. J., PAVORD, I., BRADDING, P. \& GREEN, R. H. 2012. The relationship between clinical outcomes and medication adherence in difficult-to-control asthma. Thorax, 67, 751-3.

NEDER, J., NERY, L., SILVA, A., CABRAL, A. \& FERNANDES, A. 1999. Shortterm effects of aerobic training in the clinical management of moderate to severe asthma in children. Thorax, 54, 202-6.

NEDER, J. A., NERY, L. E., PERES, C. \& WHIPP, B. J. 2001. Reference values for dynamic responses to incremental cycle ergometry in males and females aged 20 to 80. Am J Respir Crit Care Med, 164, 1481-6.

NERY, L. E., WASSERMAN, K., ANDREWS, J. D., HUNTSMAN, D. J., HANSEN, J. E. \& WHIPP, B. J. 1982. Ventilatory and gas exchange kinetics during exercise in chronic airways obstruction. J Appl Physiol Respir Environ Exerc Physiol, 53, 1594-602.

NEVILL, A. M., BATE, S. \& HOLDER, R. L. 2005. Modeling physiological and anthropometric variables known to vary with body size and other confounding variables. Am J Phys Anthropol, Suppl 41, 141-53.

NG, M., FlEMING, T., ROBINSON, M., THOMSON, B., GRAETZ, N., MARGONO, C., MULlANY, E. C., BIRYUKOV, S., ABBAFATI, C., ABERA, S. F., ABRAHAM, J. P., ABU-RMEILEH, N. M. E., ACHOKI, T., ALBUHAIRAN, F. S., ALEMU, Z. A., ALFONSO, R., ALI, M. K., ALI, R., GUZMAN, N. A., AMMAR, W., ANWARI, P., BANERJEE, A., BARQUERA, S., BASU, S., BENNETT, D. A., BHUTTA, Z., BLORE, J., CABRAL, N., NONATO, I. C., CHANG, J. C., CHOWDHURY, R., COURVILLE, K. J., CRIQUI, M. H., CUNDIFF, D. K., DABHADKAR, K. C., DANDONA, L., DAVIS, A., DAYAMA, A., DHARMARATNE, S. D., DING, E. L., DURRANI, A. M., ESTEGHAMATI, A., FARZADFAR, F., 
FAY, D. F. J., FEIGIN, V. L., FLAXMAN, A., FOROUZANFAR, M. H., GOTO, A., GREEN, M. A., GUPTA, R., HAFEZI-NEJAD, N., HANKEY, G. J., HAREWOOD, H. C., HAVMOELLER, R., HAY, S., HERNANDEZ, L., HUSSEINI, A., IDRISOV, B. T., IKEDA, N., ISLAMI, F., JAHANGIR, E., JASSAL, S. K., JEE, S. H., JEFFREYS, M., JONAS, J. B., KABAGAMBE, E. K., KHALIFA, S. E. A. H., KENGNE, A. P., KHADER, Y. S., KHANG, Y. H., KIM, D., KIMOKOTI, R. W., KINGE, J. M., KOKUBO, Y., KOSEN, S., KWAN, G., LAI, T., LEINSALU, M., LI, Y., LIANG, X., LIU, S., LOGROSCINO, G., LOTUFO, P. A., LU, Y., MA, J., MAINOO, N. K., MENSAH, G. A., MERRIMAN, T. R., MOKDAD, A. H., MOSCHANDREAS, J., NAGHAVI, M., NAHEED, A., NAND, D., NARAYAN, K. M. V., NELSON, E. L., NEUHOUSER, M. L., NISAR, M. I., OHKUBO, T., OTI, S. O., PEDROZA, A., et al. 2014. Global, regional, and national prevalence of overweight and obesity in children and adults during 1980-2013: A systematic analysis for the Global Burden of Disease Study 2013. The Lancet, 384, 766--781.

NICE 2018. Asthma. Available from: www.nice.org.uk.

NOCON, A. 1991. Social and emotional impact of childhood asthma. Arch Dis Child, 66, 458-60.

NOVOSAD, S., KHAN, S., WOLFE, B. \& KHAN, A. 2013. Role of obesity in asthma control, the obesity-asthma phenotype. J Allergy (Cairo), 2013, 538642.

NYSTAD, W. 1997. The physical activity level in children with asthma based on a survey among 7-16-year-old school children. Scandinavian Journal of Medicine \& Science in Sports, 7, 331-335.

NYSTAD, W., STIGUM, H. \& CARLSEN, K. H. 2001. Increased level of bronchial responsiveness in inactive children with asthma. Respir Med, 95, 806-10.

O'DEA, J. A. 2003. Why do kids eat healthful food? Perceived benefits of and barriers to healthful eating and physical activity among children and adolescents. J Am Diet Assoc, 103, 497-501.

O'NEILL, C. \& DOGRA, S. 2017. Subjective Responses to Interval and Continuous Exercise in Adults With Exercise-Induced Bronchoconstriction. J Phys Act Health, 14, 486-491.

OBERT, P., CleuZiOU, C., CANDAU, R., COURTEIX, D., LECOQ, A. M. \& GUENON, P. 2000. The slow component of O2 uptake kinetics during highintensity exercise in trained and untrained prepubertal children. Int J Sports Med, 21, 31-6.

ONUR, E., KABARO?LU, C., GÜNAY, O., VAR, A., YILMAZ, O., DÜNDAR, P., TIKIZ, C., GÜVENÇ, Y. \& YÜKSEL, H. 2011. The beneficial effects of physical exercise on antioxidant status in asthmatic children. Allergologia et immunopathologia, 39, 90-5.

ORTEGA, F. B., RUIZ, J. R., CASTILLO, M. J. \& SJOSTROM, M. 2008. Physical fitness in childhood and adolescence: a powerful marker of health. Int J Obes (Lond), 32, 1-11.

OWNBY, D. R., PETERSON, E. L., NELSON, D., JOSEPH, C. C., WILLIAMS, L. K. \& JOHNSON, C. C. 2007. The relationship of physical activity and percentage of body fat to the risk of asthma in 8 - to 10 -year-old children. $J$ Asthma, 44, 885-9. 
PANDITI, S. \& SILVERMAN, M. 2003. Perception of exercise induced asthma by children and their parents. Archives of Disease in Childhood, 88, 807-811.

PAPOUTSAKIS, C., PRIFTIS, K. N., DRAKOULI, M., PRIFTI, S., KONSTANTAKI, E., CHONDRONIKOLA, M., ANTONOGEORGOS, G. \& MATZIOU, V. 2013. Childhood overweight/obesity and asthma: is there a link? A systematic review of recent epidemiologic evidence. J Acad Nutr Diet, 113, 77-105.

PARSONS, J. P., HALLSTRAND, T. S., MASTRONARDE, J. G., KAMINSKY, D. A., RUNDELL, K. W., HULL, J. H., STORMS, W. W., WEILER, J. M., CHEEK, F. M., WILSON, K. C. \& ANDERSON, S. D. 2013. An official American Thoracic Society clinical practice guideline: exercise-induced bronchoconstriction. Am J Respir Crit Care Med, 187, 1016-27.

PATERSON, D. H. \& WHIPP, B. J. 1991. Asymmetries of oxygen uptake transients at the on- and offset of heavy exercise in humans. $J$ Physiol, 443, 575-86.

PAYNE, V. G., MORROW, J. R., JR., JOHNSON, L. \& DALTON, S. N. 1997. Resistance training in children and youth: a meta-analysis. Res $Q$ Exerc Sport, 68, 80-8.

PETERS-GOLDEN, M., SWERN, A., BIRD, S. S., HUSTAD, C. M., GRANT, E. \& EDELMAN, J. M. 2006. Influence of body mass index on the response to asthma controller agents. Eur Respir J, 27, 495-503.

PETERSEN, A. M. \& PEDERSEN, B. K. 2005. The anti-inflammatory effect of exercise. J Appl Physiol (1985), 98, 1154-62.

PHILPOTT, J., HOUGHTON, K. \& LUKE, A. 2010. Physical activity recommendations for children with specific chronic health conditions: Juvenile idiopathic arthritis, hemophilia, asthma and cystic fibrosis. Paediatr Child Health, 15, 213-25.

PIANOSI, P. T. \& DAVIS, H. S. 2004. Determinants of physical fitness in children with asthma. Pediatrics, 113, e225-9.

PIJNENBURG, M. W., BARALDI, E., BRAND, P. L., CARLSEN, K. H., EBER, E., FRISCHER, T., HEDLIN, G., KULKARNI, N., LEX, C., MAKELA, M. J., MANTZOURANIS, E., MOELLER, A., PAVORD, I., PIACENTINI, G., PRICE, D., ROTTIER, B. L., SAGLANI, S., SLY, P. D., SZEFLER, S. J., TONIA, T., TURNER, S., WOOLER, E. \& LODRUP CARLSEN, K. C. 2015. Monitoring asthma in children. Eur Respir J, 45, 906-25.

POOLE, D. C., BARSTOW, T. J., MCDONOUGH, P. \& JONES, A. M. 2008. Control of oxygen uptake during exercise. Med Sci Sports Exerc, 40, 462-74.

POOLE, D. C. \& JONES, A. M. 2012. Oxygen uptake kinetics. Compr Physiol, 2, 933-96.

PRICE, D., HARROW, B., SMALL, M., PIKE, J. \& HIGGINS, V. 2015. Establishing the relationship of inhaler satisfaction, treatment adherence, and patient outcomes: a prospective, real-world, cross-sectional survey of US adult asthma patients and physicians. World Allergy Organ J, 8, 26.

PROTUDJER, J. L., KOZYRSKYJ, A. L., BECKER, A. B. \& MARCHESSAULT, G. 2009. Normalization strategies of children with asthma. Qual Health Res, 19, 94-104.

PROTUDJER, J. L., MCGAVOCK, J. M., RAMSEY, C. D., SEVENHUYSEN, G. P., KOZYRSKYJ, A. L. \& BECKER, A. B. 2012. "Asthma isn't an excuse, it's just a condition": youths' perceptions of physical activity and screen time. J Asthma, 49, 496-501. 
PUENTE-MAESTU, L., SANZ, M. L., SANZ, P., RUIZ DE ONA, J. M., RODRIGUEZ-HERMOSA, J. L. \& WHIPP, B. J. 2000. Effects of two types of training on pulmonary and cardiac responses to moderate exercise in patients with COPD. Eur Respir J, 15, 1026-32.

RABE, K. F., VERMEIRE, P. A., SORIANO, J. B. \& MAIER, W. C. 2000. Clinical management of asthma in 1999: the Asthma Insights and Reality in Europe (AIRE) study. Eur Respir J, 16, 802-7.

RACIL, G., COQUART, J., ELMONTASSAR, W., HADDAD, M., GOEBEL, R., CHAOUACHI, A., AMRI, M. \& CHAMARI, K. 2016. Greater effects of high- compared with moderate-intensity interval training on cardio-metabolic variables, blood leptin concentration and ratings of perceived exertion in obese adolescent females. Biol Sport.

RALEIGH, J. P., GILES, M. D., SCRIBBANS, T. D., EDGETT, B. A., SAWULA, L. J., BONAFIGLIA, J. T., GRAHAM, R. B. \& GURD, B. J. 2016. The impact of work-matched interval training on VO2peak and VO2 kinetics: diminishing returns with increasing intensity. Appl Physiol Nutr Metab, 41, 706-13.

RAM, F., ROBINSON, S. \& BLACK, P. 2000. Effects of physical training in asthma: a systematic review. British Journal of Sports Medicine, 34, 162-167.

RAM, F. S., ROBINSON, S. M., BLACK, P. N. \& PICOT, J. 2005. Physical training for asthma. Cochrane Database Syst Rev, Cd001116.

RASMUSSEN, F., LAMBRECHTSEN, J., SIERSTED, H. C., HANSEN, H. S. \& HANSEN, N. C. 2000. Low physical fitness in childhood is associated with the development of asthma in young adulthood: the Odense schoolchild study. Eur Respir J, 16, 866-70.

RATEL, S. \& WILLIAMS, C. A. 2017. Neuromuscular fatigue. In: ARMSTRONG, N. \& VAN MECHELEN, W. (eds.) Oxford textbook of children's sport and exercise medicine Oxford, UK: Oxford University Press.

RAUNER, A., MESS, F. \& WOLL, A. 2013. The relationship between physical activity, physical fitness and overweight in adolescents: a systematic review of studies published in or after 2000. BMC Pediatr, 13, 19.

REDDEL, H. K., TAYLOR, D. R., BATEMAN, E. D., BOULET, L. P., BOUSHEY, H. A., BUSSE, W. W., CASALE, T. B., CHANEZ, P., ENRIGHT, P. L., GIBSON, P. G., DE JONGSTE, J. C., KERSTJENS, H. A., LAZARUS, S. C., LEVY, M. L., O'BYRNE, P. M., PARTRIDGE, M. R., PAVORD, I. D., SEARS, M. R., STERK, P. J., STOLOFF, S. W., SULLIVAN, S. D., SZEFLER, S. J., THOMAS, M. D. \& WENZEL, S. E. 2009. An official American Thoracic Society/European Respiratory Society statement: asthma control and exacerbations: standardizing endpoints for clinical asthma trials and clinical practice. Am J Respir Crit Care Med, 180, 59-99.

REICHERT, F. F., BARROS, A. J., DOMINGUES, M. R. \& HALLAL, P. C. 2007. The role of perceived personal barriers to engagement in leisure-time physical activity. Am J Public Health, 97, 515-9.

RHEE, H., WENZEL, J. \& STEEVES, R. H. 2007. Adolescents' psychosocial experiences living with asthma: a focus group study. J Pediatr Health Care, 21, 99-107.

RICH, C., GERACI, M., GRIFFITHS, L., SERA, F., DEZATEUX, C. \& CORTINA-BORJA, M. 2013. Quality control methods in accelerometer data processing: defining minimum wear time. PLoS One, 8, e67206. 
RIDGERS, N. D., BARNETT, L. M., LUBANS, D. R., TIMPERIO, A., CERIN, E. \& SALMON, J. 2018a. Potential moderators of day-to-day variability in children's physical activity patterns. $J$ Sports $S c i, 36,637-644$.

RIDGERS, N. D., KNOWLES, Z. R. \& SAYERS, J. 2012. Encouraging play in the natural environment: a child-focused case study of Forest School. Children's Geographies, 10, 49-65.

RIDGERS, N. D., TIMPERIO, A., BROWN, H., BALL, K., MACFARLANE, S., LAI, S. K., RICHARDS, K., MACKINTOSH, K. A., MCNARRY, M. A., FOSTER, M. \& SALMON, J. 2018b. Wearable Activity Tracker Use Among Australian Adolescents: Usability and Acceptability Study. JMIR Mhealth Uhealth, 6, e86.

RIETVELD, S., PRINS, P. J. M. \& COLLAND, V. T. 2010. Accuracy of Symptom Perception in Asthma and Illness Severity. Children's Health Care, 30, 2741.

RINER, W. F. \& SELLHORST, S. H. 2013. Physical activity and exercise in children with chronic health conditions. Journal of Sport and Health Science, 2, 12-20.

RODRIGO, G. J. \& PLAZA, V. 2007. Body mass index and response to emergency department treatment in adults with severe asthma exacerbations: a prospective cohort study. Chest, 132, 1513-9.

RODRIGUES, A. N., PEREZ, A. J., CARLETTI, L., BISSOLI, N. S. \& ABREU, G. R. 2006. Maximum oxygen uptake in adolescents as measured by cardiopulmonary exercise testing: a classification proposal. J Pediatr (Rio J), 82, 426-30.

ROSENTHAL, M., BAIN, S. H., CRAMER, D., HELMS, P., DENISON, D., BUSH, A. \& WARNER, J. O. 1993. Lung function in white children aged 4 to 19 years: I--Spirometry. Thorax, 48, 794-802.

ROSS, L. M., PORTER, R. R. \& DURSTINE, J. L. 2016. High-intensity interval training (HIIT) for patients with chronic diseases. Journal of Sport and Health Science, 5, 139-144.

ROSS, R. M. 2003. ATS/ACCP statement on cardiopulmonary exercise testing. Am J Respir Crit Care Med. United States.

ROWLAND, T. W., UNNITHAN, V. B., MACFARLANE, N. G., GIBSON, N. G. \& PATON, J. Y. 1994. Clinical manifestations of the 'athlete's heart' in prepubertal male runners. Int J Sports Med, 15, 515-9.

RUDELL, K., HAREENDRAN, A., BONNER, N., ARBUCKLE, R., BURBRIDGE, C. \& ABETZ, L. 2012. Patients' experience of asthma control and clinical guidelines: perspectives from a qualitative study. Respir Med, 106, 909-11.

SACHS-OLSEN, C., BERNTSEN, S., LODRUP CARLSEN, K. C., ANDERSSEN, S. A., MOWINCKEL, P. \& CARLSEN, K. H. 2013. Time spent in vigorous physical activity is associated with increased exhaled nitric oxide in nonasthmatic adolescents. Clin Respir J, 7, 64-73.

SAGLANI, S. \& BUSH, A. 2008. Asthma, Atopy, and Airway Inflammation. American Journal of Respiratory and Critical Care Medicine, 178, 437-438.

SAINT-PIERRE, P., BOURDIN, A., CHANEZ, P., DAURES, J. P. \& GODARD, P. 2006. Are overweight asthmatics more difficult to control? Allergy, 61, 7984. 
SANTUZ, P., BARALDI, E., FILIPPONE, M. \& ZACCHELLO, F. 1997. Exercise performance in children with asthma: is it different from that of healthy controls? Eur Respir J, 10, 1254-60.

SAVAS, M., WESTER, V. L., STAUFENBIEL, S. M., KOPER, J. W., VAN DEN AKKER, E. L. T., VISSER, J. A., VAN DER LELY, A. J., PENNINX, B. \& VAN ROSSUM, E. F. C. 2017. Systematic Evaluation of Corticosteroid Use in Obese and Non-obese Individuals: A Multi-cohort Study. Int J Med Sci, $14,615-621$.

SAWYER, S. M. \& FARDY, H. J. 2003. Bridging the gap between doctors' and patients' expectations of asthma management. J Asthma, 40, 131-8.

SAYNOR, Z. L., BARKER, A. R., OADES, P. J. \& WILLIAMS, C. A. 2016. Impaired Pulmonary V O2 Kinetics in Cystic Fibrosis Depend on Exercise Intensity. Med Sci Sports Exerc, 48, 2090-2099.

SHERRIFF, A., MAITRA, A., NESS, A. R., MATTOCKS, C., RIDDOCH, C., REILLY, J. J., PATON, J. Y. \& HENDERSON, A. J. 2009. Association of duration of television viewing in early childhood with the subsequent development of asthma. Thorax, 64, 321-5.

SIDIROPOULOU, M., FOTIADOU, E., TSIMARAS, V., ZAKAS, A. \& ANGELOPOULOU, N. 2007. The effect of interval training in children with exercise-induced asthma competing in soccer. Journal of strength and conditioning research, 21, 446-50.

SIRICHANA, W., TON, Y. N. \& COOPER, C. B. 2014. Characteristics of the ventilatory response to incremental exercise in asthma. European respiratory journal, 44.

SLY, R., HARPER, R. \& ROSSELOT, I. 1972. The effect of physical conditioning upon asthmatic children. Annals of allergy, 30, 86-94.

SOUSA, A. W., CABRAL, A. L., MARTINS, M. A. \& CARVALHO, C. R. 2014. Daily physical activity in asthmatic children with distinct severities. $J$ Asthma, 51, 493-7.

SPATHOPOULOS, D., PARASKAKIS, E., TRYPSIANIS, G., TSALKIDIS, A., ARVANITIDOU, V., EMPORIADOU, M., BOUROS, D. \& CHATZIMICHAEL, A. 2009. The effect of obesity on pulmonary lung function of school aged children in Greece. Pediatr Pulmonol, 44, 273-80.

SPYCHER, B. D., SILVERMAN, M. \& KUEHNI, C. E. 2010. Phenotypes of childhood asthma: are they real? Clin Exp Allergy, 40, 1130-41.

STEWART, A., MARFELL-JONES, M., OLDS, T. \& DE RIDDER, H. 2011. International standards for anthropometric assessment, Lower Hutt, NZ, ISAK.

SUMI, Y. \& HAMID, Q. 2007. Airway remodeling in asthma. Allergol Int, 56, 3418.

SUNDBOM, F., MALINOVSCHI, A., LINDBERG, E., ALVING, K. \& JANSON, C. 2016. Effects of poor asthma control, insomnia, anxiety and depression on quality of life in young asthmatics. J Asthma, 53, 398-403.

SUTHERLAND, T. J., COWAN, J. O., YOUNG, S., GOULDING, A., GRANT, A. M., WILLIAMSON, A., BRASSETT, K., HERBISON, G. P. \& TAYLOR, D. R. 2008. The association between obesity and asthma: interactions between systemic and airway inflammation. Am J Respir Crit Care Med, 178, 469-75. 
SWALLOW, E. B., GOSKER, H. R., WARD, K. A., MOORE, A. J., DAYER, M. J., HOPKINSON, N. S., SCHOLS, A. M., MOXHAM, J. \& POLKEY, M. I. 2007. A novel technique for nonvolitional assessment of quadriceps muscle endurance in humans. J Appl Physiol (1985), 103, 739-46.

SWANN, I. \& HANSON, C. 1983. Double-blind prospective study of the effect of physical training on childhood asthma. The Asthmatic Child - In Play and Sport, 318-25.

SZEFLER, S. J., MARTIN, R. J., KING, T. S., BOUSHEY, H. A., CHERNIACK, R. M., CHINCHILLI, V. M., CRAIG, T. J., DOLOVICH, M., DRAZEN, J. M., FAGAN, J. K., FAHY, J. V., FISH, J. E., FORD, J. G., ISRAEL, E., KILEY, J., KRAFT, M., LAZARUS, S. C., LEMANSKE, R. F., JR., MAUGER, E., PETERS, S. P. \& SORKNESS, C. A. 2002. Significant variability in response to inhaled corticosteroids for persistent asthma. $J$ Allergy Clin Immunol, 109, 410-8.

SZEFLER, S. J., PHILlIPS, B. R., MARTINEZ, F. D., CHINCHILlI, V. M., LEMANSKE, R. F., STRUNK, R. C., ZEIGER, R. S., LARSEN, G., SPAHN, J. D., BACHARIER, L. B., BLOOMBERG, G. R., GUILBERT, T. W., HELDT, G., MORGAN, W. J., MOSS, M. H., SORKNESS, C. A. \& TAUSSIG, L. M. 2005. Characterization of within-subject responses to fluticasone and montelukast in childhood asthma. J Allergy Clin Immunol, $115,233-42$.

TANAKA, H., MONAHAN, K. D. \& SEALS, D. R. 2001. Age-predicted maximal heart rate revisited. J Am Coll Cardiol, 37, 153-6.

TAPPE, M. K., DUDA, J. L. \& EHRNWALD, P. M. 1989. Perceived Barriers To Exercise Among Adolescents. Journal of School Health, 59, 153-155.

TAYLOR, D. R., BATEMAN, E. D., BOULET, L. P., BOUSHEY, H. A., BUSSE, W. W., CASAlE, T. B., CHANEZ, P., ENRIGHT, P. L., GIBSON, P. G., DE JONGSTE, J. C., KERSTJENS, H. A., LAZARUS, S. C., LEVY, M. L., O'BYRNE, P. M., PARTRIDGE, M. R., PAVORD, I. D., SEARS, M. R., STERK, P. J., STOLOFF, S. W., SZEFLER, S. J., SULLIVAN, S. D., THOMAS, M. D., WENZEL, S. E. \& REDDEL, H. K. 2008. A new perspective on concepts of asthma severity and control. Eur Respir J, 32, 545-54.

TAYLOR, K. L., WESTON, M. \& BATTERHAM, A. M. 2015. Evaluating intervention fidelity: an example from a high-intensity interval training study. PLoS One, 10, e0125166.

TAYLOR, W. R. \& NEWACHECK, P. W. 1992. Impact of childhood asthma on health. Pediatrics, 90, 657-62.

THORNTON, J. 1997. Overcoming 'protected child syndrome': kids, exercise, and chronic illness. Phys Sportsmed, 25, 97-100.

TJONNA, A. E., STOLEN, T. O., BYE, A., VOLDEN, M., SLORDAHL, S. A., ODEGARD, R., SKOGVOLL, E. \& WISLOFF, U. 2009. Aerobic interval training reduces cardiovascular risk factors more than a multitreatment approach in overweight adolescents. Clin Sci (Lond), 116, 317-26.

TOENNESEN, L. L., SOERENSEN, E. D., HOSTRUP, M., PORSBJERG, C., BANGSBO, J. \& BACKER, V. 2018. Feasibility of high-intensity training in asthma. Eur Clin Respir J, 5, 1468714. 
TOLFREY, K., CAMPBELL, I. G. \& BATTERHAM, A. M. 1998. Aerobic Trainability of Prepubertal Boys and Girls. Pediatric Exercise Science, 10, 248-263.

TOWNSEND, N., BHATNAGAR, P., WICKRAMASINGHE, K., WILLIAMS, J., VUJCICH, D. \& RAYNER, M. 2013. Children and young people statistics 2013. Department of public health.

TROLlVIK, A., NORDBACH, R., SILEN, C. \& RINGSBERG, K. C. 2011. Children's experiences of living with asthma: fear of exacerbations and being ostracized. J Pediatr Nurs, 26, 295-303.

TROST, S. G., KERR, L. M., WARD, D. S. \& PATE, R. R. 2001. Physical activity and determinants of physical activity in obese and non-obese children. Int $J$ Obes Relat Metab Disord, 25, 822-9.

TROST, S. G., LOPRINZI, P. D., MOORE, R. \& PFEIFFER, K. A. 2011. Comparison of accelerometer cut points for predicting activity intensity in youth. Med Sci Sports Exerc, 43, 1360-8.

TSAI, S. Y., WARD, T., LENTZ, M. J. \& KIECKHEFER, G. M. 2012. Daytime physical activity levels in school-age children with and without asthma. Nurs Res, 61, 252-9.

TSAKIRIS, A., IORDANIDOU, M., PARASKAKIS, E., TSALKIDIS, A., RIGAS, A., ZIMERAS, S., KATSARDIS, C. \& CHATZIMICHAEL, A. 2013. The presence of asthma, the use of inhaled steroids, and parental education level affect school performance in children. Biomed Res Int, 2013, 762805.

TURNER-WARWICK, M. 1989. Nocturnal asthma: a study in general practice. $J R$ Coll Gen Pract, 39, 239-43.

TURNER, S., EASTWOOD, P., COOK, A. \& JENKINS, S. 2010. Improvements in symptoms and quality of life following exercise training in older adults with moderate/severe persistent asthma. Respiration, 22, 1-9.

ULGER, Z., DEMIR, E., TANAC, R., GOKSEN, D., GULEN, F., DARCAN, S., CAN, D. \& COKER, M. 2006. The effect of childhood obesity on respiratory function tests and airway hyperresponsiveness. Turk J Pediatr, 48, 43-50.

UNNITHAN, V. B., ROCHE, D. M., GARRARD, M., HOLLOWAY, K. \& MARWOOD, S. 2015. Oxygen uptake kinetics in trained adolescent females. Eur J Appl Physiol, 115, 213-20.

URQUHART, D. S. \& VENDRUSCULO, F. M. 2017. Clinical interpretation of cardiopulmonary exercise testing in cystic fibrosis and implications for exercise counselling. Paediatr Respir Rev, 24, 72-78.

VAHLKVIST, S., INMAN, M. D. \& PEDERSEN, S. 2010. Effect of asthma treatment on fitness, daily activity and body composition in children with asthma. Allergy, 65, 1464-71.

VAHLKVIST, S. \& PEDERSEN, S. 2009. Fitness, daily activity and body composition in children with newly diagnosed, untreated asthma. Allergy, 64, 1649-55.

VAN DEN BEMT, L., KOOIJMAN, S., LINSSEN, V., LUCASSEN, P., MURIS, J., SLABBERS, G. \& SCHERMER, T. 2010. How does asthma influence the daily life of children? Results of focus group interviews. Health Qual Life Outcomes, 8.

VAN GENT, R., VAN DER ENT, C. K., VAN ESSEN-ZANDVLIET, L. E., ROVERS, M. M., KIMPEN, J. L., DE MEER, G. \& KLIJN, P. H. 2007. No 
differences in physical activity in (un)diagnosed asthma and healthy controls. Pediatr Pulmonol, 42, 1018-23.

VAN HUISSTEDE, A., CASTRO CABEZAS, M., VAN DE GEIJN, G. J., MANNAERTS, G. H., NJO, T. L., TAUBE, C., HIEMSTRA, P. S. \& BRAUNSTAHL, G. J. 2013. Underdiagnosis and overdiagnosis of asthma in the morbidly obese. Respir Med, 107, 1356-64.

VAN LEEUWEN, J. C., DRIESSEN, J. M., DE JONGH, F. H., VAN AALDEREN, W. M. \& THIO, B. J. 2011. Monitoring pulmonary function during exercise in children with asthma. Arch Dis Child, 96, 664-8.

VAN LEEUWEN, J. C., HOOGSTRATE, M., DUIVERMAN, E. J. \& THIO, B. J. 2014. Effects of dietary induced weight loss on exercise-induced bronchoconstriction in overweight and obese children. Pediatr Pulmonol, 49, 1155-61.

VAN SLUIJS, E. M., MCMINN, A. M. \& GRIFFIN, S. J. 2007. Effectiveness of interventions to promote physical activity in children and adolescents: systematic review of controlled trials. Bmj, 335, 703.

VAN VELDHOVEN, N. H., VERMEER, A., BOGAARD, J. M., HESSELS, M. G., WIJNROKS, L., COLLAND, V. T. \& VAN ESSEN-ZANDVLIET, E. E. 2001. Children with asthma and physical exercise: effects of an exercise programme. Clin Rehabil, 15, 360-70.

VARNI, J. W., BURWINKLE, T. M. \& SEID, M. 2006. The PedsQL 4.0 as a school population health measure: feasibility, reliability, and validity. Qual Life Res, 15, 203-15.

VARNI, J. W., BURWINKLE, T. M., SEID, M. \& SKARR, D. 2003. The PedsQL 4.0 as a pediatric population health measure: feasibility, reliability, and validity. Ambul Pediatr, 3, 329-41.

VARNI, J. W., SEID, M. \& KURTIN, P. S. 2001. PedsQL 4.0: reliability and validity of the Pediatric Quality of Life Inventory version 4.0 generic core scales in healthy and patient populations. Med Care, 39, 800-12.

VARNI, J. W., SEID, M. \& RODE, C. A. 1999. The PedsQL: measurement model for the pediatric quality of life inventory. Med Care, 37, 126-39.

VARRAY, A., MERCIER, J. \& PREFAUT, C. 1995. Individualized training reduces excessive exercise hyperventilation in asthmatics. International journal of rehabilitation research., 18, 297-312.

VARRAY, A. L., MERCIER, J. G., TERRAL, C. M. \& PREFAUT, C. G. 1991. Individualized aerobic and high intensity training for asthmatic children in an exercise readaptation program. Is training always helpful for better adaptation to exercise? CHEST, 99, 579-586.

VILLA, F., CASTRO, A. P., PASTORINO, A. C., SANTAREM, J. M., MARTINS, M. A., JACOB, C. M. \& CARVALHO, C. R. 2011. Aerobic capacity and skeletal muscle function in children with asthma. Arch Dis Child. England.

VRIJENS, B., DE GEEST, S., HUGHES, D. A., PRZEMYSLAW, K., DEMONCEAU, J., RUPPAR, T., DOBBELS, F., FARGHER, E., MORRISON, V., LEWEK, P., MATYJASZCZYK, M., MSHELIA, C., CLYNE, W., ARONSON, J. K. \& URQUHART, J. 2012. A new taxonomy for describing and defining adherence to medications. Br J Clin Pharmacol, 73, 691-705.

WALDERS-ABRAMSON, N., WAMBOLDT, F. S., CURRAN-EVERETT, D. \& ZHANG, L. 2009. Encouraging physical activity in pediatric asthma: a case- 
control study of the wonders of walking (WOW) program. Pediatr Pulmonol, 44, 909-16.

WALKER, T. J. \& REZNIK, M. 2014. In-school asthma management and physical activity: children's perspectives. J Asthma, 51, 808-13.

WALSH, T. R., IRWIN, D. E., MEIER, A., VARNI, J. W. \& DEWALT, D. A. 2008. The use of focus groups in the development of the PROMIS pediatrics item bank. Qual Life Res, 17, 725-35.

WANG, J. \& HUNG, W. 2009. The effects of a swimming intervention for children with asthma. Respirology (Carlton, Vic.) [Online], 14. Available: http://onlinelibrary.wiley.com/o/cochrane/clcentral/articles/532/CN00733532/frame.html.

WANG, Y. 2004. Epidemiology of childhood obesity--methodological aspects and guidelines: what is new? Int J Obes Relat Metab Disord, 28 Suppl 3, S21-8.

WANROOIJ, V. H., WILLEBOORDSE, M., DOMPELING, E. \& VAN DE KANT, K. D. 2014. Exercise training in children with asthma: a systematic review. Br J Sports Med, 48, 1024-31.

WARBURTON, D. E., MCKENZIE, D. C., HAYKOWSKY, M. J., TAYLOR, A., SHOEMAKER, P., IGNASZEWSKI, A. P. \& CHAN, S. Y. 2005. Effectiveness of high-intensity interval training for the rehabilitation of patients with coronary artery disease. Am J Cardiol, 95, 1080-4.

WEILER, J. M., BONINI, S., COIFMAN, R., CRAIG, T., DELGADO, L., CAPAOFILIPE, M., PASSALI, D., RANDOLPH, C., STORMS, W., AD HOC COMMITTEE OF SPORTS MEDICINE COMMITTEE OF AMERICAN ACADEMY OF ALLERGY, A. \& IMMUNOLOGY 2007. American Academy of Allergy, Asthma \& Immunology Work Group report: exerciseinduced asthma. J Allergy Clin Immunol, 119, 1349-58.

WELCH, N., MCNAUGHTON, S. A., HUNTER, W., HUME, C. \& CRAWFORD, D. 2009. Is the perception of time pressure a barrier to healthy eating and physical activity among women? Public Health Nutr, 12, 888-95.

WELSH, L., KEMP, J. G. \& ROBERTS, R. G. D. 2005. Effects of physical conditioning on children and adolescents with asthma. Sports Medicine, 35, 127-141.

WELSH, L., ROBERTS, R. G. \& KEMP, J. G. 2004. Fitness and physical activity in children with asthma. Sports Med, 34, 861-70.

WENZEL, S. E. 2012. Asthma phenotypes: the evolution from clinical to molecular approaches. Nat Med, 18, 716-25.

WESTERGREN, T., FEGRAN, L., NILSEN, T., HARALDSTAD, K., KITTANG, O. B. \& BERNTSEN, S. 2016. Active play exercise intervention in children with asthma: a PILOT STUDY. BMJ Open, 6, e009721.

WESTON, A. R., MACFARLANE, D. J. \& HOPKINS, W. G. 1989. Physical activity of asthmatic and nonasthmatic children. J Asthma, 26, 279-86.

WESTON, K. L., AZEVEDO, L. B., BOCK, S., WESTON, M., GEORGE, K. P. \& BATTERHAM, A. M. 2016. Effect of Novel, School-Based High-Intensity Interval Training (HIT) on Cardiometabolic Health in Adolescents: Project FFAB (Fun Fast Activity Blasts) - An Exploratory Controlled Before-AndAfter Trial. PLoS One, 11, e0159116.

WESTON, K. S., WISLOFF, U. \& COOMBES, J. S. 2014a. High-intensity interval training in patients with lifestyle-induced cardiometabolic disease: a systematic review and meta-analysis. Br J Sports Med, 48, 1227-34. 
WESTON, M., TAYLOR, K. L., BATTERHAM, A. M. \& HOPKINS, W. G. 2014b. Effects of low-volume high-intensity interval training (HIT) on fitness in adults: A meta-analysis of controlled and non-controlled trials. Sports Medicine, 44, 1005--1017.

WHIPP, B. J., DAVIS, J. A., TORRES, F. \& WASSERMAN, K. 1981. A test to determine parameters of aerobic function during exercise. $J$ Appl Physiol Respir Environ Exerc Physiol, 50, 217-21.

WHIPP, B. J. \& WARD, S. A. 1990. Physiological determinants of pulmonary gas exchange kinetics during exercise. Med Sci Sports Exerc, 22, 62-71.

WHIPP, B. J. \& WASSERMAN, K. 1972. Oxygen uptake kinetics for various intensities of constant-load work. J Appl Physiol, 33, 351-6.

WHO 2018a. Obesity. Available from: www.who.int.

WHO 2018b. Physical activity. Available from: www.who.int.

WICHER, I., RIBEIRO, M., MARMO, D., SANTOS, C., TORO, A., MENDES, R., CIELO, F. \& RIBEIRO, J. 2010. Effects of swimming on spirometric parameters and bronchial hyperresponsiveness in children and adolescents with moderate persistent atopic asthma. Jornal de pediatria, 86, 384-90.

WILCOX, S. L., BROXTERMAN, R. M. \& BARSTOW, T. J. 2016. Constructing quasi-linear VO2 responses from nonlinear parameters. J Appl Physiol (1985), 120, 121-9.

WILLEBOORDSE, M., VAN DE KANT, K. D. G., VAN DER VELDEN, C. A., VAN SCHAYCK, C. P. \& DOMPELING, E. 2016. Associations between asthma, overweight and physical activity in children: a cross-sectional study. BMC Public Health, 16, 919.

WILLIAMS, B., HOSKINS, G., POW, J., NEVILLE, R., MUKHOPADHYAY, S. \& COYLE, J. 2010. Low exercise among children with asthma: a culture of over protection? A qualitative study of experiences and beliefs. $\mathrm{Br} J$ Gen Pract, 60, e319-26.

WILLIAMS, B., POWELL, A., HOSKINS, G. \& NEVILLE, R. 2008a. Exploring and explaining low participation in physical activity among children and young people with asthma: a review. BMC Family Practice, 9, 11p.

WILliAMS, B., POWELL, A., HOSKINS, G. \& NEVILLE, R. 2008b. Exploring and explaining low participation in physical activity among children and young people with asthma: a review. BMC Fam Pract. England.

WILLIAMS, L. K., PETERSON, E. L., WELLS, K., AHMEDANI, B. K., KUMAR, R., BURCHARD, E. G., CHOWDHRY, V. K., FAVRO, D., LANFEAR, D. E. \& PLADEVALL, M. 2011. Quantifying the proportion of severe asthma exacerbations attributable to inhaled corticosteroid nonadherence. J Allergy Clin Immunol, 128, 1185-1191.e2.

WILSON, D. K., WILliAMS, J., EVANS, A., MIXON, G. \& RHEAUME, C. 2005. Brief report: a qualitative study of gender preferences and motivational factors for physical activity in underserved adolescents. J Pediatr Psychol, 30, 293-7.

WINLOVE, M. A., JONES, A. M. \& WELSMAN, J. R. 2010. Influence of training status and exercise modality on pulmonary $\mathrm{O}(2)$ uptake kinetics in prepubertal girls. Eur J Appl Physiol, 108, 1169-79.

WRIGHT, A., LAVOIE, K. L., JACOB, A., RIZK, A. \& BACON, S. L. 2010. Effect of body mass index on self-reported exercise-triggered asthma. Phys Sportsmed, 38, 61-6. 
YIALLOUROS, P. K., ECONOMOU, M., KOLOKOTRONI, O., SAVVA, S. C., GAVATHA, M., IOANNOU, P., KARPATHIOS, T. \& MIDDLETON, N. 2015. Gender differences in objectively assessed physical activity in asthmatic and non-asthmatic children. Pediatr Pulmonol, 50, 317-26.

YOSHIDA, T. \& WHIPP, B. J. 1994. Dynamic asymmetries of cardiac output transients in response to muscular exercise in man. J Physiol, 480 ( Pt 2), $355-9$. 
APPENDICES 


\section{APPENDIX A:}

\section{INFORMATION SHEETS AND CONSENT FORMS}




\title{
Appendices
}

\section{Appendix A: Information sheets and consent forms}

\section{Head teacher information sheets}

\author{
Applied Sports Technology Exercise and Medicine Research Centre (A-STEM) \\ Sport and Health Portfolio, College of Engineering
}

HEADTEACHER INFORMATION SHEET (Intervention) (Version 1.4, Date: 25/06/2015)

Project Title:

The $\mathrm{x} 4 \mathrm{a}$ trial: eXercise for Asthma with Commando Joes.

Contact Details:

William Eddolls

Charles Winn

Dr Kelly Mackintosh

Dr Melitta McNarry

Phone:

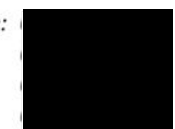

1. Invitation Paragraph

Thank you for taking the time to read this information sheet, it will provide you with the details of our study and hopefully provide you with the information you require to help you decide if you want your school to participate. It is important to say at this point that the decision to take part is entirely up to you and that your school will not be at a disadvantage for future studies should you decide not to participate.

2. What is the purpose of the study?

A strong relationship has been shown between physical inactivity and obesity. Worryingly only a small portion of children meet the government guidelines of 60 minutes of exercise. This, in a lot of cases, is because time constraints do not allow children to get the exercise they need. Short bouts of high intensity exercise have previously been shown to be an effective and useful way of meeting exercise requirements. Therefore, the purpose of this study is to assess whether high intensity training can be used as an effective method of improving numerous physical and mental health factors including asthma symptoms and quality of life.

3. Why has your school been chosen?

Your school has been selected via random assignment after being selected as a potentially suitable school. The results from the initial questionnaire will help us identify pupils with asthma and we would therefore like to involve them within the study.

However this does not mean your schools has to take part in the study. This is voluntary and you have the right to withdraw your school from the study at any time. If you wish to withdraw from the study you will not be hassled, get in trouble, and you will not have to give a reason for your withdrawal. All participants will be able to withdraw themselves from the study at any point without fear of penalty on withdrawal. 


\section{What will happen to your pupils if they take part?}

Once consent/assent is returned students will be invited to attend our exercise programme. This is a

45 minute exercise session that includes 30 minutes of high intensity exercise, conducted 3 times a week before or after school. These sessions will alter weekly and last for 6 months. Over the course of the intervention this totals approximately 54 hours. An example session would be planned as follows:

Warm-up - This will last approximately 5 minutes. In the warm-up the students will take part in light jogging and stretches to ensure their body is ready to exercise.

High intensity exercise - This will consist of numerous bouts of 30 second exercises followed by 30 seconds rest. Exercises will include numerous military style games. This section of the programme will last 30 minutes.

Warm-down - This will be conducted to ensure that the children experience minimal muscle soreness following exercise. This will last 10 minutes to allow for possible asthma related issues.

All pupils will also be invited to an assessment day where a number of tests will be conducted. These assessment days will occur at 5 time points across approximately 12 months but should only require the students to miss half a day of lessons per time point. This will total approximately 25 hours. These tests will include:

Questionnaires - These will include quality of life, mental well-being, asthma health utilization, asthma control and an anxiety questionnaire, we will also ask each asthmatic pupil to keep a short diary including any GP or hospital visits for asthma.

Lung function test and fractional exhaled nitric oxide - Participants will be asked to blow hard into one device and gently into a tube connected to a monitor, these will be taken 5 times throughout the year.

Maturation assessments - The children will be asked to complete a questionnaire. It consists of showing line drawings of different pubertal stages. A box next to each picture is then ticked by the child to identify which pubertal stage resembles them. This will be done in complete privacy and only looked at by researchers on completion of the study. Please see attached sheet for line drawings.

Multi-stage fitness test - The children will be asked to complete a bleep test to assess their fitness at different points throughout the year. It will require the children to run $20 \mathrm{~m}$ shuttle runs to the sound of beeps which increase in speed until the child no longer reaches the line before the beep sounds.

Focus groups - In this the children will be interview in groups of 4-6 and asked questions related to exercise and asthma. These sessions will be either videoed or voice recorded. Those that are randomly selected to attend will be asked to provide us with 1 hour of their time. 
Physical activity measurement - Children will be asked to wear a physical activity monitor for 7 consecutive days. They will also be asked to keep an activity log to record when they remove the accelerometer. Handing out and collecting the accelerometers will take approximately 1 hour at 5 time points over 12 months, totaling 5 hours.

A smaller group of children will also be selected for the laboratory testing (roughly 16 per school [8 asthmatic and 8 non-asthmatic]). They will be asked to attend a laboratory session over 4 days (this will be a maximum of 40 mins each session) at 5 time points. These also can be run in your school. During this laboratory testing students will be asked to complete tests that will include:

Functional fitness test - In this test they will practice using the static bicycle until they are happy with it. It will start off easy and get harder until your child says they can't keep going. During this, they will breath into a mask to allow us to measure the air that they breathe in and out. This mask does not make breathing any harder and you can talk through it and remove it at any time they fee uncomfortable about wearing it. A device will also be placed on the upper leg. The exercise will last approximately 15 minutes.

Blood analysis - A small pin prick will be administered to the children's finger where an extremely small drop of blood will be taken, if the children do not feel comfortable doing this test then they will be able to decline without any question.

Blood pressure and heart rate - Participants will be asked to place an inflatable cuff around their arm. This will inflate to momentarily slow the blood flow to the arm to measure blood pressure. Once the measurement is collected the cuff will be removed. To measure heart rate, a monitor will be worn around the student's chest. This is an elastic belt that fits all. An area will be designated to allow the student's to attach this in private.

Pulse Wave Velocity - in order to measure the health of the children's arteries we will measure the speed at which blood pressure waves flow. In order to measure this we will need to apply a cuff around the neck and upper leg of the children. This measurement should only last 5 minutes.

5. What are the possible disadvantages of taking part?

There aren't any significant risks or discomforts within the study. If your pupils follow our instructions which will ensure that you are appropriately warm for the activity then the risks will be minimised. There is a small risk of injury from the activity (as in any Physical Education class); however there will be trained first aiders on hand to deal with any injuries which may occur. Asthmatics taking part in the study may succumb to exercise induced asthma after the exercise is complete; this is the main reason for the warm down as if this does occur it will be roughly 7 minutes after the main part of the programme. If this does occur we have personnel trained in asthma care in this situation that would be able to support the child appropriately with inhaler use.

6. What are the possible benefits of taking part?

Your student's physical fitness and mental wellbeing including your student's quality of life could improve through completing this program. It will also help provide crucial answers to research questions in the health and exercise area of study namely asthma, childhood obesity and other 
effects of physical inactivity.

7. Will my pupils taking part in the study be kept confidential?

Although your pupils' details will be taken prior to conducting the study, they will remain anonymous and this would be the same should the study be published. Your pupil's personal information will be stored on a protected computer with a password and personal information will be destroyed at the study end (with consent forms stored in a locked cabinet in a secure area, to be destroyed within 5 years of study end). Members of the university will be able to access your information under supervision.

8. What if I have any questions?

If you have any problems or need any further information about the study or the testing then please contact any of the research team using the contact details provided. 
Applied Sports Technology Exercise and Medicine Research Centre (A-STEM)

Sport and Health Portfolio, College of Engineering

Swansea University
Prifysgol Abertawe

\section{HEADTEACHER INFORMATION SHEET (Control)}

(Version 1.4, Date: 25/06/2015)

Project Title:

The x4a trial: eXercise for Asthma with Commando Joes.

Contact Details:

William Eddolls

Charles Winn

Dr Kelly Mackintosh

Dr Melitta McNarry

Phone:

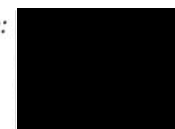

Email:

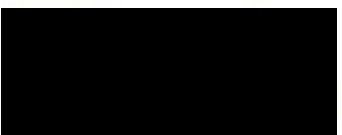

1. Invitation Paragraph

Thank you for taking the time to read this information sheet, it will provide you with the details of our study and hopefully provide you with the information you require to help you decide if you want your school to participate. It is important to say at this point that the decision to take part is entirely up to you and that your school will not be at a disadvantage for future studies should you decide for them not to participate.

\section{What is the purpose of the study?}

A strong relationship has been shown between physical inactivity and obesity. Worryingly only a small portion of children meet the government guidelines of 60 minutes of exercise. This, in a lot of cases, is because time constraints do not allow children to get the exercise they need. Short bouts of high intensity exercise have previously been shown to be an effective and useful way of meeting exercise requirements. Therefore, the purpose of this study is to assess whether high intensity training can be used as an effective method of improving numerous physical and mental health factors including asthma symptoms and quality of life.

3. Why has your school been chosen?

Your school has been selected via random assignment to be a control school. This will mean that similar measures are made on the children as in the intervention schools where the exercise programme is being delivered so that we can tell if the programme has made a difference. However this does not mean your school has to take part in the study. This is voluntary and you have the right to withdraw your school from the study at any time. If you wish to withdraw from the study you will not be contacted further, and you will not have to give a reason for your schools withdrawal. All participants will be able to withdraw themselves from the study at any point without fear of penalty on withdrawal. 
4. What will happen to your pupils if they take part?

Once consent/assent is returned your students all pupils will also be invited to an assessment day where a number of tests will be conducted. These assessment days will occur at 5 time points across approximately 12 months but should only require the students to miss half a day of lessons per time point (these days will be run at your school). These tests will include:

Questionnaires - These will include quality of life, mental well-being, asthma health utilization, asthma control and an anxiety questionnaire, we will also ask each asthmatic pupil to keep a short diary including any GP or hospital visits for asthma.

Lung function test and fractional exhaled nitric oxide - Participants will be asked to blow hard into one device and gently into a tube connected to a monitor.

Maturation assessments - The children will be asked to complete a questionnaire. It consists of showing line drawings of different pubertal stages. A box next to each picture is then ticked by the child to identify which pubertal stage resembles them. This will be done in complete privacy and only looked at by researchers on completion of the study.

Multi-stage fitness test - The children will be asked to complete a bleep test to assess their fitness at different points throughout the year. It will require the children to run $20 \mathrm{~m}$ shuttle runs to the sound of beeps which increase in speed until the child no longer reaches the line before the beep sounds.

Physical activity measurement - Children will be asked to wear a physical activity monitor for 7 consecutive days. They will also be asked to keep an activity log to record when they remove the accelerometer. Handing out and collecting the accelerometers will take approximately 1 hour at 5 time points over 12 months, totaling 5 hours.

A smaller group of children will also be selected for the laboratory testing (roughly 16 per school [8 asthmatic and 8 non-asthmatic]). They will be asked to attend a laboratory session over 4 days (this will be a maximum of 40 mins each session) at 5 time points. These also can be run in your school. During this laboratory testing students will be asked to complete tests that will include:

Functional fitness test - In this test they will practice using the static bicycle until they are happy with it. It will start off easy and get harder until your child says they can't keep going. During this, they will breath into a mask to allow us to measure the air that they breathe in and out. This mask does not make breathing any harder and you can talk through it and remove it at any time they feel uncomfortable about wearing it. A device will also be placed on the upper leg. The exercise will last approximately 15 minutes.

Blood analysis - A small pin prick will be administered to the children's finger where an extremely 
small drop of blood will be taken, if the children do not feel comfortable doing this test then they will be able to decline without any question.

Blood pressure and heart rate - Participants will be asked to place an inflatable cuff around their arm. This will inflate to momentarily slow the blood flow to the arm to measure blood pressure. Once the measurement is collected the cuff will be removed. To measure heart rate, a monitor wil be worn around the student's chest. This is an elastic belt that fits all. An area will be designated to allow the student's to attach this in private.

5. What are the possible disadvantages of taking part?

There aren't any significant risks or discomforts within the study. If your pupils follow our instructions which will ensure that you are appropriately warm for the activity then the risks will be minimised. There is a small risk of injury from the activity (as in any Physical Education class); however there will be trained first aiders on hand to deal with any injuries which may occur. Asthmatics taking part in the study may succumb to exercise induced asthma after the exercise is complete; this is the main reason for the warm down as if this does occur it will be roughly 7 minutes after the main part of the programme. If this does occur we have personnel trained in asthma care in this situation that would be able to support the child appropriately with inhaler use.

6. What are the possible benefits of taking part?

Your student's physical fitness and mental wellbeing including your student's quality of life could improve through completing this program. It will also help provide crucial answers to research questions in the health and exercise area of study namely asthma, childhood obesity and other effects of physical inactivity.

7. Will my pupils taking part in the study be kept confidential?

Although your pupils' details will be taken prior to conducting the study, they will remain anonymous and this would be the same should the study be published. Your pupil's personal information will be stored on a protected computer with a password and personal information will be destroyed at the study end (with consent forms stored in a locked cabinet in a secure area, to be destroyed within 5 years of study end). Members of the university will be able to access your information under supervision.

8. What if I have any questions?

If you have any problems or need any further information about the study or the testing then please contact any of the research team using the contact details provided. 


\section{Head teacher consent form}

Applied Sports Technology Exercise and Medicine Research Centre (A-STEM) Sport and Health Portfolio, College of Engineering

HEADTEACHER CONSENT FORM

(Version 1.4, Date: 25/06/2015)

Project Title:

The x4a trial: eXercise for Asthma with Commando Joes.

Contact Details:

William Eddolls

Charles Winn

Dr Kelly Mackintosh

Dr Melitta McNarry
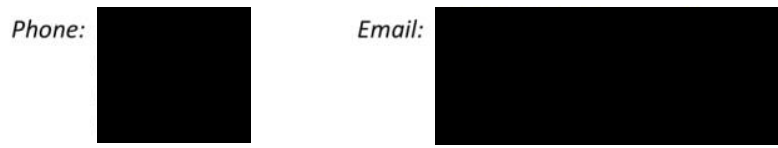

Please initial box

1. I confirm that I have read and understood the information sheet dated 25/06/2015 (version number 1.4) for the above study and have had the opportunity to ask questions.

2. I understand that my school's participation is voluntary and that I am free to withdraw at any time, without giving any reason, without my legal rights being affected.

3. I understand that sections of any of data obtained may be looked at by responsible individuals from the Swansea University or from research regulatory authorities where it is relevant to my taking part in research. I give permission for these individuals to have access to these records. Analysis will be done on anonymous data.

4. I give consent for video and voice recording and photography. (providing parent/guardian have provided consent)

5. I agree for my school to take part in the above study.

Name of School

Name of Headteacher

Researcher
Date

Date
Signature

Signature 


\title{
Parental/guardian information sheets
}

\author{
Applied Sports Technology Exercise and Medicine Research Centre (A-STEM) \\ Sport and Health Portfolio, College of Engineering
}

PARENTAL/GUARDIAN INFORMATION SHEET (Intervention) (Version 1.4, Date: 25/06/2015)

Project Title:

The x4a trial: eXercise for Asthma with Commando Joes.

Contact Details:

William Eddolls

Charles Winn

Dr Kelly Mackintosh

Dr Melitta McNarry

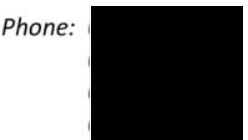

Email:

1. Invitation Paragraph

Thank you for taking the time to read this information sheet, it will provide you with the details of our study and hopefully provide you with the information you require to help you decide if you want your child to participate. It is important to say at this point that the decision to take part is entirely up to you and that your child will not be at a disadvantage for future studies should you decide for them not to participate.

2. What is the purpose of the study?

A link has been shown between exercise and obesity. Worryingly only a small number of children meet the government guidelines of 60 minutes of exercise. This, a lot of the time, is because time does not allow children to get the exercise they need. High intensity exercise has been shown to be a good way of getting enough exercise. So, we want to see whether high intensity training can be used to improve mental and physical health including asthma and quality of life.

\section{Why has your child been chosen?}

Your child's school has been chosen randomly. However this does not mean your child has to take part in the study. This is voluntary and you have the right to withdraw them from the study at any time. If you wish to withdraw your child from the study you will not be hassled, and you will not have to give a reason for their withdrawal. Your child will also be able to withdraw themselves from the study at any point without fear of penalty on withdrawal.

4. What will happen to your child if they take part?

Your child will be invited to attend our exercise program. This is a 45 minute exercise session that includes 30 minutes of high intensity exercise conducted 3 times a week before or after school over 6 months. Over the course of the intervention this totals approximately 54 hours. These sessions will alter weekly. An example session would be planned as follows: 
Warm-up - This will last approximately 5 minutes. In the warm-up the children will take part in light jogging and stretches to ensure their body is ready to exercise.

High intensity exercise - This will consist of numerous bouts of 30 second exercises followed by 30 seconds rest. Exercises will include numerous military style games. This section of the programme will last 30 minutes.

Warm-down - This will be conducted to ensure that the children experience minimal muscle soreness following exercise. This will last 10 minutes to allow for possible asthma related issues.

Your child will also be invited to an assessment day where a number of tests will be conducted. These assessment days will occur at 5 time points across approximately 12 months but should only require your child to miss half a day of lessons per time point. These tests will include:

Questionnaires - These will include quality of life, mental well-being, asthma health utilization, asthma control and an anxiety questionnaire, we will also ask each asthmatic pupil to keep a short diary including any GP or hospital visits for asthma.

Lung function test and fractional exhaled nitric oxide - Participants will be asked to blow hard into one device and gently into a tube connected to a monitor, these will be taken 5 times throughout the year.

Maturation assessments - The children will be asked to complete a questionnaire. It consists of showing line drawings of different pubertal stages. A box next to each picture is then ticked by the child to identify which pubertal stage resembles them. This will be done in complete privacy and only looked at by researchers on completion of the study. Please see attached sheet for line drawings.

Multi-stage fitness test - Your child will be asked to complete a bleep test to assess their fitness at different points throughout the year. It will require them to run $20 \mathrm{~m}$ shuttle runs to the sound of beeps which increase in speed until they no longer reach the line before the beep sounds.

Focus groups - In this the children will be interview in groups of 4-6 and asked questions related to exercise and asthma. These sessions will be either videoed or voice recorded. Those that are randomly selected to attend will be asked to provide us with 1 hour of their time.

Physical activity measurement - Children will be asked to wear a physical activity monitor for 7 consecutive days. They will also be asked to keep an activity log to record when they remove the accelerometer. Handing out and collecting the accelerometers will take approximately 1 hour at 5 time points over 12 months, totaling 5 hours.

Your child may also be selected for the laboratory testing (roughly 16 per school [ 8 asthmatic and 8 non-asthmatic]). They will be asked to attend a laboratory session over 4 days (this will be a maximum of 40 mins each session) at 5 time points. These also can be run in your school. During this laboratory testing the children will be asked to complete a number of tests that will include: 
Functional fitness test - In this test they will practice using the static bicycle until they are happy with it. It will start off easy and get harder until your child says they can't keep going. During this, they will breath into a mask to allow us to measure the air that they breathe in and out. This mask does not make breathing any harder and you can talk through it and remove it at any time they feel uncomfortable about wearing it. A device will also be placed on the upper leg. The exercise will last approximately 15 minutes.

Blood analysis - A small pin prick will be administered to the children's finger where an extremely small drop of blood will be taken, if your child does not feel comfortable doing this test then they will be able to decline without any question.

Blood pressure and heart rate - The children will be asked to place an inflatable cuff around their arm. This will inflate to momentarily slow the blood flow to the arm to measure blood pressure. Once the measurement is collected the cuff will be removed. To measure heart rate, a monitor wil be worn around your child's chest. This is an elastic belt that fits all. An area will be designated to allow your child to attach this in private.

Pulse Wave Velocity - in order to measure the health of your child's arteries we will measure the speed at which blood pressure waves flow. In order to measure this we will need to apply a cuff around the neck and upper leg of your child. This measurement should only last 5 minutes.

5. What are the possible disadvantages of taking part?

There aren't any significant risks or discomforts within the study. If your child follows our instructions which will ensure that they are appropriately warm for the activity then the risks will be minimised. There is a small risk of injury from the activity (as in any Physical Education class) however there will be trained first aiders on hand to deal with any injuries which may occur. Asthmatics taking part in the study may succumb to exercise induced asthma after the exercise is complete; this is the main reason for the warm down as if this does occur it will be roughly 7 minutes after the main part of the programme. If this does occur we have personnel trained in asthma care in this situation that would be able to support the child appropriately with inhaler use.

6. What are the possible benefits of taking part?

Your child may find it interesting to know what some of their measurements are e.g. their lung functions, fitness, physical fitness and mental wellbeing including your child's quality of life could improve through completing this program. The study is also likely to help provide crucial answers to research questions in the health and exercise area of study namely asthma, childhood obesity and other effects of inactivity.

7. Will my child taking part in the study be kept confidential?

Although your child's details will be taken prior to conducting the study, they will remain anonymous and this would be the same should the study be published. Your child's personal information will be stored on a protected computer with a password and personal information will be destroyed at the study end (with consent forms stored in a locked cabinet in a secure area, to be destroyed within 5 years of study end). Members of the university will be able to access your information under 
supervision

8. What if I have any questions?

If you have any problems or need any further information about the study or the testing then please contact any of the research team using the contact details provided. If you have concerns or questions regarding the programme but do not want to contact the research team directly, please contact your child's school tutor. 


\section{PARENTAL/GUARDIAN INFORMATION SHEET (Control)}

(Version 1.4, Date: 25/06/2015)

Project Title:

The $\mathrm{x} 4 \mathrm{a}$ trial: eXercise for Asthma with Commando Joes.

\section{Contact Details:}

William Eddolls

Charles Winn

Dr Kelly Mackintosh

Dr Melitta McNarry

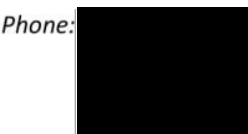

Email:

1. Invitation Paragraph

Thank you for taking the time to read this information sheet, it will provide you with the details of our study and hopefully provide you with the information you require to help you decide if you want your child to participate. It is important to say at this point that the decision to take part is entirely up to you and that your child will not be at a disadvantage for future studies should you decide for them not to participate.

2. What is the purpose of the study?

A link has been shown between exercise and obesity. Worryingly only a small number of children meet the government guidelines of 60 minutes of exercise. This, a lot of the time, is because time does not allow children to get the exercise they need. High intensity exercise has been shown to be a good way of getting enough exercise. So, we want to see whether high intensity training can be used to improve mental and physical health including asthma and quality of life.

3. Why has your child been chosen?

Your child's school has been chosen randomly to be a control school. This will mean that similar measures are made on the children as in the intervention schools where the exercise programme is being delivered so that we can tell if the programme has made a difference. However this does not mean your child has to take part in the study. This is voluntary and you have the right to withdraw them from the study at any time. If you wish to withdraw your child from the study you will not be hassled, and you will not have to give a reason for their withdrawal. Your child will also be able to withdraw themselves from the study at any point without fear of penalty on withdrawal.

4. What will happen to your child if they take part?

Your child will be invited to an assessment day where a number of tests will be conducted. These 
assessment days will occur at 5 time points across approximately 12 months but should only require your child to miss half a day of lessons per time point. These tests will include:

Questionnaires - These will include quality of life, mental well-being, asthma health utilization and a physical self-perception questionnaire, we will also ask each asthmatic pupil to keep a short diary including any GP or hospital visits for asthma.

Lung function test and fractional exhaled nitric oxide - Participants will be asked to blow hard into device and gently into a tube connected to a monitor, these will be taken 5 times throughout the year.

Maturation assessments - The children will be asked to complete a questionnaire. It consists of showing line drawings of different pubertal stages. A box next to each picture is then ticked by the child to identify which pubertal stage resembles them. This will be done in complete privacy and only looked at by researchers on completion of the study. Please see attached sheet for line drawings.

Multi-stage fitness test - Your child will be asked to complete a bleep test to assess their fitness at different points throughout the year. It will require them to run $20 \mathrm{~m}$ shuttle runs to the sound of beeps which increase in speed until they no longer reach the line before the beep sounds.

Physical activity measurement - Children will be asked to wear a physical activity monitor for 7 consecutive days. They will also be asked to keep an activity log to record when they remove the accelerometer. Handing out and collecting the accelerometers will take approximately 1 hour at 5 time points over 12 months, totaling 5 hours.

Your child may also be selected for the laboratory testing (roughly 16 per school [8 asthmatic and 8 non-asthmatic]). They will be asked to attend a laboratory session over 4 days (this will be a maximum of 40 mins each session) at 5 time points. These also can be run in your school. During this laboratory testing the children will be asked to complete a number of tests that will include:

Functional fitness test - In this test they will practice using the static bicycle until they are happy with it. It will start off easy and get harder until your child says they can't keep going. During this, they will breath into a mask to allow us to measure the air that they breathe in and out. This mask does not make breathing any harder and you can talk through it and remove it at any time they feel uncomfortable about wearing it. A device will also be placed on the upper leg. The exercise will last approximately 15 minutes.

Blood analysis - A small pin prick will be administered to the children's finger where an extremely small drop of blood will be taken, if your child does not feel comfortable doing this test then they will be able to decline without any question.

Blood pressure and heart rate - The children will be asked to place an inflatable cuff around their arm. This will inflate to momentarily slow the blood flow to the arm to measure blood pressure. 
Once the measurement is collected the cuff will be removed. To measure heart rate, a monitor wil be worn around your child's chest. This is an elastic belt that fits all. An area will be designated to allow your child to attach this in private.

\section{What are the possible disadvantages of taking part?}

There aren't any significant risks or discomforts within the study. If your child follows our instructions which will ensure that they are appropriately warm for the activity then the risks will be minimised. There is a small risk of injury from the activity (as in any Physical Education class); however there will be trained first aiders on hand to deal with any injuries which may occur. Asthmatics taking part in the study may succumb to exercise induced asthma after the exercise is complete; this is the main reason for the warm down as if this does occur it will be roughly 7 minutes after the main part of the programme. If this does occur we have personnel trained in asthma care in this situation that would be able to support the child appropriately with inhaler use.

6. What are the possible benefits of taking part?

Your child may find it interesting to know what some of their measurements are e.g. their lung functions, fitness, physical fitness and mental wellbeing including your child's quality of life. The study is also likely to help provide crucial answers to research questions in the health and exercise area of study namely asthma, childhood obesity and other effects of inactivity.

7. Will my child taking part in the study be kept confidential?

Although your child's details will be taken prior to conducting the study, they will remain anonymous and this would be the same should the study be published. Your child's personal information will be stored on a protected computer with a password and personal information will be destroyed at the study end (with consent forms stored in a locked cabinet in a secure area, to be destroyed within 5 years of study end). Members of the university will be able to access your information under supervision.

8. What if I have any questions?

If you have any problems or need any further information about the study or the testing then please contact any of the research team using the contact details provided. If you have concerns or questions regarding the programme but do not want to contact the research team directly, please contact your child's school tutor. 


\section{Parental/guardian consent form}

Applied Sports Technology Exercise and Medicine Research Centre (A-STEM)

Sport and Health Portfolio, College of Engineering

Swansea University
Prifysgol Abertawe

PARENTAL/GUARDIAN CONSENT FORM

(Version 1.4, Date: 25/06/2015)

Project Title:

The x4a trial: eXercise for Asthma with Commando Joes.

Contact Details:

William Eddolls

Charles Winn

Dr Kelly Mackintosh

Dr Melitta McNarry
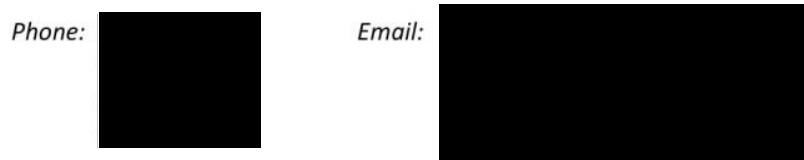

Please initial box

1. I confirm that I have read and understood the information sheet dated 25/06/2015 (version number 1.4) for the above study and have had the opportunity to ask questions.

2. I understand that my participation is voluntary and that I am free to withdraw at any time, without giving any reason, without my legal rights being affected.

3. I understand that sections of any of data obtained may be looked at by responsible individuals from the Swansea University or from regulatory authorities where it is relevant to my taking part in research. I give permission for these individuals to have access to these records. Analysis will be done on anonymous data.

4. I give consent for video and voice recording (discussion groups) and Photography.

5. Does your child have asthma, as confirmed by a doctor? Yes/No

6. I agree for my child to take part in the above study.

Name of Child

Name of Parent/Guardian

Date

Signature

Researcher

Date

Signature 


\section{Participant information sheets}

Applied Sports Technology Exercise and Medicine Research Centre (A-STEM)

Sport and Health Portfolio, College of Engineering

Swansea University
Prifysgol Abertawe

\section{PARTICIPANT'S INFORMATION SHEET (Intervention)}

(Version 1.4, Date: 25/06/2015)

Project Title:

The $\mathrm{x} 4 \mathrm{a}$ trial: eXercise for Asthma with Commando Joes.

Contact Details:

William Eddolls

Charles Winn

Phone: 07786910861

Dr Kelly Mackintosh

07812608957

01792295075

Email: 636442@swansea.ac.uk

552067@swansea.ac.uk

k.mackintosh@swansea.ac.uk

Dr Melitta McNarry

01792513069

m.mcnarry@swansea.ac.uk

1. Invitation Paragraph

You are being invited to take part in a research study. Before you decide it is important for you to understand why the research is being done and what it will involve. Please take time to read the following information carefully and discuss it with others if you wish. Ask us if there is anything that is not clear or if you would like more information. If you want to stop taking part in this testing at anytime just tell one of the researchers.

2. What is the purpose of the study?

A link has been shown between obesity and lack of exercise. Worryingly only a few children meet guidelines that suggest that children and teenagers get 60 minutes of exercise per day. This study is looking into exercise and how it can affect health both mentally and physically in secondary school children in the South Wales. This is so that we can try and improve mental and physical health of children your age across Wales and the U.K.

\section{Why have I been chosen?}

You been chosen because you fit the example this study wishes to look at. You are between the ages of $11-14$ years.

However this does not mean you have to take part in the study. Meaning you can withdraw from the study at any time without fear of penalty on withdrawal. If you want to withdraw you will not have to give a reason. Just let one of us know.

4. What will happen to if you take part?

Your will be invited to attend our exercise program. This is a 45 minute exercise session that includes 30 minutes of high intensity exercise conducted 3 times a week before or after school over 6 months. Over the course of the intervention this totals approximately 54 hours. These sessions will 
alter weekly. An example session would be planned as follows:

Warm-up - This will last approximately 5 minutes. In the warm-up you will take part in light jogging and stretches to ensure their body is ready to exercise.

High intensity exercise - This will consist of numerous bouts of 30 second exercises followed by 30 seconds rest. Exercises will include numerous military style games. This section of the programme will last 30 minutes.

Warm-down - This will be conducted to ensure that you experience minimal muscle soreness following exercise. This will last 10 minutes to allow for possible asthma related issues.

You will be invited to an assessment day where a number of tests will be conducted. These assessment days will occur at 5 time points across approximately 12 months but should only require you to miss half a day of lessons per time point. These tests will include:

Questionnaires - These will include quality of life, mental well-being, asthma health utilization, asthma control and an anxiety questionnaire, we will also ask each asthmatic pupil to keep a short diary including any GP or hospital visits for asthma.

Lung function test and fractional exhaled nitric oxide - You will be asked to blow hard into one device and gently into a tube connected to a monitor, these will be taken 5 times throughout the year.

Maturation assessments - You will be asked to complete a questionnaire. It consists of showing line drawings of different pubertal stages. A box next to each picture is then ticked by yourself to identify which pubertal stage resembles you. This will be done in complete privacy and only looked at by researchers on completion of the study. Please see attached sheet for line drawings.

Multi-stage fitness test - You will be asked to complete a bleep test to assess their fitness at different points throughout the year. It will require you to run $20 \mathrm{~m}$ shuttle runs to the sound of beeps which increase in speed until you can no longer reach the line before the beep sounds.

Breathing Mask

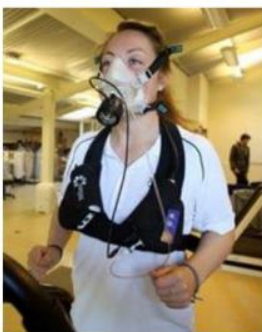

Focus groups - You will be interview in groups of 4-6 and asked questions related to exercise and asthma. These sessions will be either videoed or voice recorded. Those that are randomly selected to attend will be asked to provide us with 1 hour of their time.

Physical activity measurement - You will be asked to wear a physical activity monitor for 7 days. You will also be asked to keep an activity log to record when they remove the monitor. Handing out and collecting the accelerometers will take approximately 1 hour at 5 time points over 12 months, totaling 5 hours.

You may also be selected for the laboratory testing (roughly 16 per schoo 
[ 8 asthmatic and 8 non-asthmatic]). You will be asked to attend a laboratory session over 4 days (this will be a maximum of 40 mins each session) at 5 time points. These also can be run in your school. In these laboratory tests you will be asked to complete a number of tests that will include:

Functional fitness test - In this test you will practice using the static bicycle until you are happy with it. It will start off easy and get harder until your say you can't keep going. During this, you will breath into a mask to allow us to measure the air that you breathe in and out. This mask does not make breathing any harder and you can talk through it and remove it at any time you feel uncomfortable about wearing it. A device will also be placed on the upper leg. The exercise will last approximately 15 minutes.

Blood analysis - A small pin prick will be administered to your finger where an extremely small drop of blood will be taken, if you do not feel comfortable doing this test then you will be able to decline without any question.

Blood pressure and heart rate - You will be asked to place an inflatable cuff around your arm. This will inflate to momentarily slow the blood flow to the arm to measure blood pressure. Once the measurement is collected the cuff will be removed. To measure heart rate, a monitor will be worn around your chest. This is an elastic belt that fits all. An area will be designated to allow you to attach this in private.

Pulse Wave Velocity - in order to measure the health of your arteries we will measure the speed at which blood pressure waves flow. In order to measure this we will need to apply a cuff around your neck and upper leg. This measurement should only last 5 minutes.

5. What are the possible disadvantages of taking part?

There aren't any real risks or discomforts within the study. If you follow our instructions which will ensure that you are warmed up for the activity then the risks will be reduced. There is a small risk of injury from the activity (as in any Physical Education class); however there will be trained first aiders on hand to deal with any injuries which may occur. Asthmatics taking part in the study may succumb to exercise induced asthma after the exercise is complete. However this is unlikely. This is the main reason for the warm down as if this does occur it will be roughly 7 minutes after the main part of the programme. If this does occur we have trained personnel who will be able to care for you.

6. What are the possible benefits of taking part?

You may find it interesting to know what some of your measurements are e.g. your lung functions, fitness. Physical fitness and mental wellbeing including your quality of life could improve through completing this program. The study is also likely to help provide crucial answers to research questions in the health and exercise area of study namely asthma, childhood obesity and other effects of inactivity.

7. Will my taking part in the study be kept confidential?

Yes. Although your details will be collected before the start of the study, you will remain nameless and this will be the same if they study is published. Your personal information will be stored on a 
protected computer with a password. Only members of the university will be able to access your information under strict supervision.

8. What if I have any questions?

If you have any problems or need any further information about the study or the testing then please contact Charlie or Tom on the two first addresses at the start of the pack. If you have concerns or questions regarding the programme but do not want to contact the research team directly, please contact your school tutor. 
Applied Sports Technology Exercise and Medicine Research Centre (A-STEM) Sport and Health Portfolio, College of Engineering

PARTICIPANT'S INFORMATION SHEET (Control)

(Version 1.4, Date: 25/06/2015)

Project Title:

The $x 4 a$ trial: eXercise for Asthma with Commando Joes.

Contact Details:

William Eddolls

Charles Winn

Dr Kelly Mackintosh

Dr Melitta McNarry

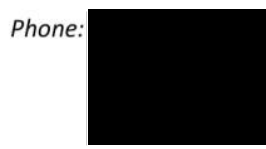

Email:

1. Invitation Paragraph

You are being invited to take part in a research study. Before you decide it is important for you to understand why the research is being done and what it will involve. Please take time to read the following information carefully and discuss it with others if you wish. Ask us if there is anything that is not clear or if you would like more information. If you want to stop taking part in this testing at anytime just tell one of the researchers.

2. What is the purpose of the study?

A link has been shown between obesity and lack of exercise. Worryingly only a few children meet guidelines that suggest that children and teenagers get 60 minutes of exercise per day. This study is looking into exercise and how it can affect health both mentally and physically in secondary schoo children in the South Wales. This is so that we can try and improve mental and physical health of children your age across Wales and the U.K.

3. Why have I been chosen?

You been chosen because you fit the example this study wishes to look at. You are between the ages of $11-14$ years.

However this does not mean you have to take part in the study. Meaning you can withdraw from the study at any time without fear of penalty on withdrawal. If you want to withdraw you will not have to give a reason. Just let one of us know.

4. What will happen to if you take part?

You will be invited to an assessment day where a number of tests will be conducted. These assessment days will occur at 5 time points across approximately 12 months but should only require you to miss half a day of lessons per time point. These tests will include: 
Questionnaires - These will include quality of life, mental well-being, asthma health utilization, asthma control and an anxiety questionnaire, we will also ask each asthmatic pupil to keep a short diary including any GP or hospital visits for asthma.

Lung function test and fractional exhaled nitric oxide - You will be asked to blow hard into one device and gently into a tube connected to a monitor, these will be taken 5 times throughout the year.

Multi Stage Fitness Test - You will be asked to complete a bleep test to assess their fitness at different points throughout the year. It will require you to run $20 \mathrm{~m}$ shuttle runs to the sound of beeps which increase in speed until you can no longer reach the line before the beep sounds.

Maturation assessments - You will be asked to complete a questionnaire. It consists of Static Cycle showing line drawings of different pubertal stages. A box next to each picture is then ticked by yourself to identify which pubertal stage resembles you. This will be done in complete privacy and only looked at by researchers on completion of the study. Please see attached sheet for line drawings.

Physical activity measurement - You will be asked to wear a physical activity monitor for 7 days. You will also be asked to keep an activity log to record when they remove the monitor. Handing out and collecting the accelerometers will take approximately 1 hour at 5 time points over 12 months, totaling 5 hours.

You may also be selected for the laboratory testing (roughly 16 per school $[8$ asthmatic and 8 nonasthmatic]). You will be asked to attend a laboratory session over 4 days (this will be a maximum of 40 mins each session) at 5 time points. These also can be run in your school. In these laboratory tests you will be asked to complete a number of tests that will include:

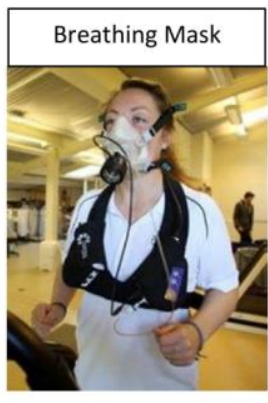

Functional fitness test - In this test you will practice using the static bicycle until you are happy with it. It will start off easy and get harder until your say you can't keep going. During this, you will breath into a mask to allow us to measure the air that you breathe in and out. This mask does not make breathing any harder and you can talk through it and remove it at any time you feel uncomfortable about wearing it. A device will also be placed on the upper leg. The exercise will last approximately 15 minutes.

Blood analysis - A small pin prick will be administered to your finger where an extremely small drop of blood will be taken, if you do not feel comfortable doing this test then you will be able to decline without any question.

Blood pressure and heart rate - You will be asked to place an inflatable cuff around your arm. This 
will inflate to momentarily slow the blood flow to the arm to measure blood pressure. Once the measurement is collected the cuff will be removed. To measure heart rate, a monitor will be worn around your chest. This is an elastic belt that fits all. An area will be designated to allow you to attach this in private.

\section{What are the possible disadvantages of taking part?}

There aren't any real risks or discomforts within the study. If you follow our instructions which will ensure that you are warmed up for the activity then the risks will be reduced. There is a small risk of injury from the activity (as in any Physical Education class); however there will be trained first aiders on hand to deal with any injuries which may occur. Asthmatics taking part in the study may succumb to exercise induced asthma after the exercise is complete. However this is unlikely. This is the main reason for the warm down as if this does occur it will be roughly 7 minutes after the main part of the programme. If this does occur we have trained personnel who will be able to care for you.

6. What are the possible benefits of taking part?

You may find it interesting to know what some of your measurements are e.g. your lung functions, fitness. The study is also likely to help provide crucial answers to research questions in the health and exercise area of study namely asthma, childhood obesity and other effects of inactivity.

7. Will my taking part in the study be kept confidential?

Yes. Although your details will be collected before the start of the study, you will remain nameless and this will be the same if they study is published. Your personal information will be stored on a protected computer with a password. Only members of the university will be able to access your information under strict supervision.

8. What if I have any questions?

If you have any problems or need any further information about the study or the testing then please contact Charlie or Tom on the two first addresses at the start of the pack. If you have concerns or questions regarding the programme but do not want to contact the research team directly, please contact your school tutor. 


\section{Participant assent form}

Applied Sports Technology Exercise and Medicine Research Centre (A-STEM)

Sport and Health Portfolio, College of Engineering

Swansea University
Prifysgol Abertawe

\section{PARTICIPANT'S ASSENT FORM}

(Version 1.4, Date: 25/06/2015)

Project Title:

The x4a trial: eXercise for Asthma with Commando Joes.

Contact Details:

William Eddolls

Charles Winn

Dr Kelly Mackintosh

Dr Melitta McNarry
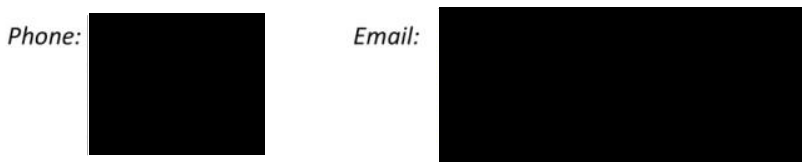

Please initial box

1. I confirm that I have read and understood the information sheet dated 25/06/2015 (version number 1.4) for the above study and have had the opportunity to ask questions.

2. I understand that my participation is voluntary and that I am free to withdraw at any time, without giving any reason, without my medical care or legal rights being affected.

3. I understand that sections of any of data obtained may be looked at by responsible individuals from the Swansea University or from regulatory authorities where it is relevant to my taking part in research. I give permission for these individuals to have access to these records. Analysis will be done on anonymous data.

4. I give consent for video and voice recording and photography.

5. Do you have asthma, as confirmed by a doctor?

Yes/No

6. I agree to take part in the above study.

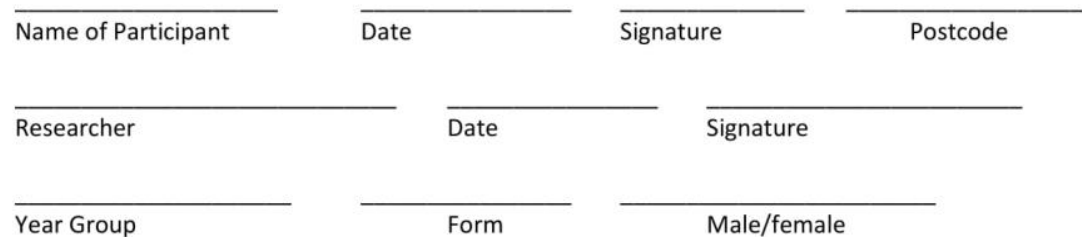




\section{APPENDIX B:}

TANNER STAGES 


\section{Appendix B: Self-report Tanner stage scale}

$\square$ Tick the box that most closely resembles yourself.
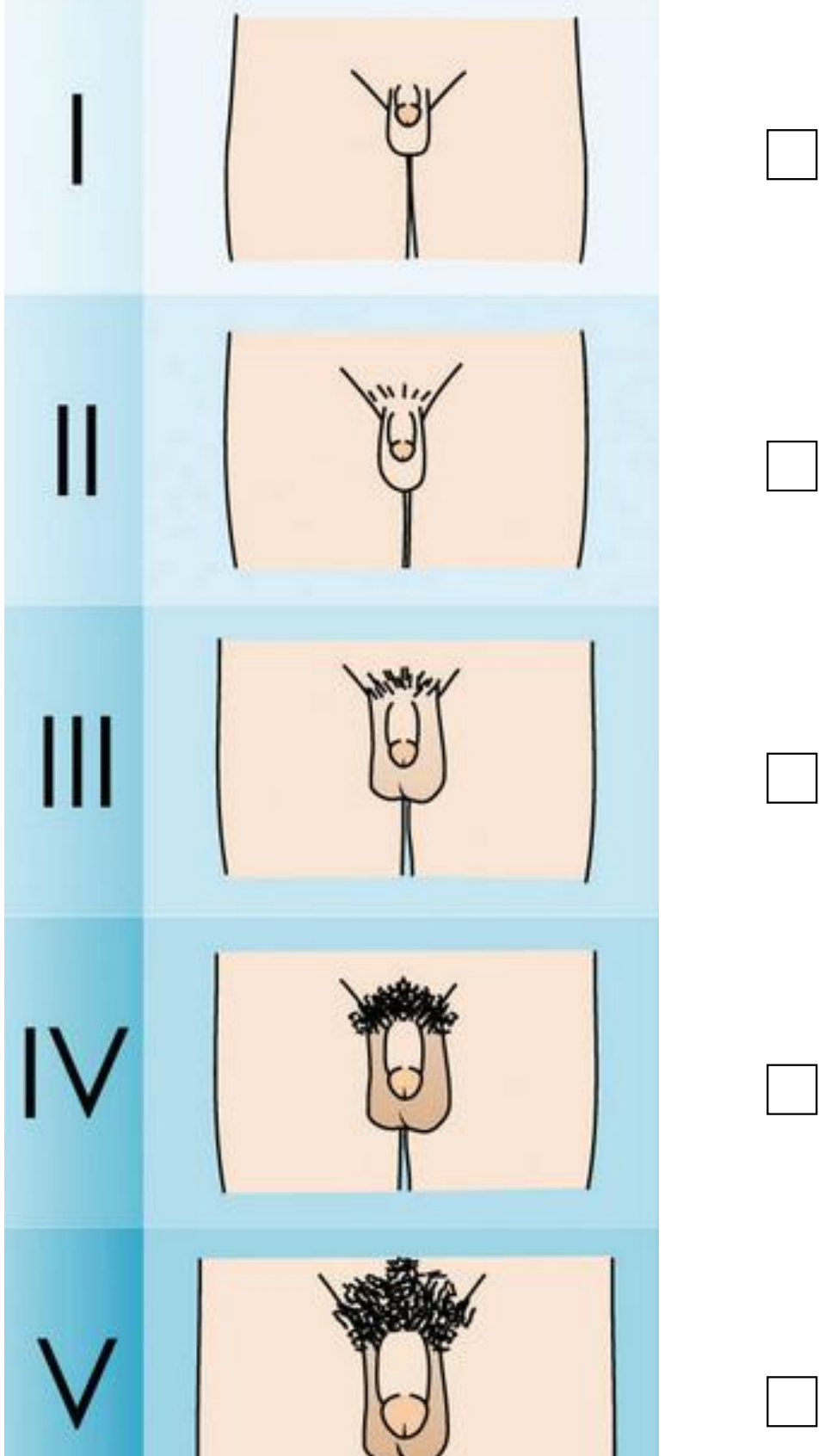
$\square$ Tick the box that most closely resembles yourself.

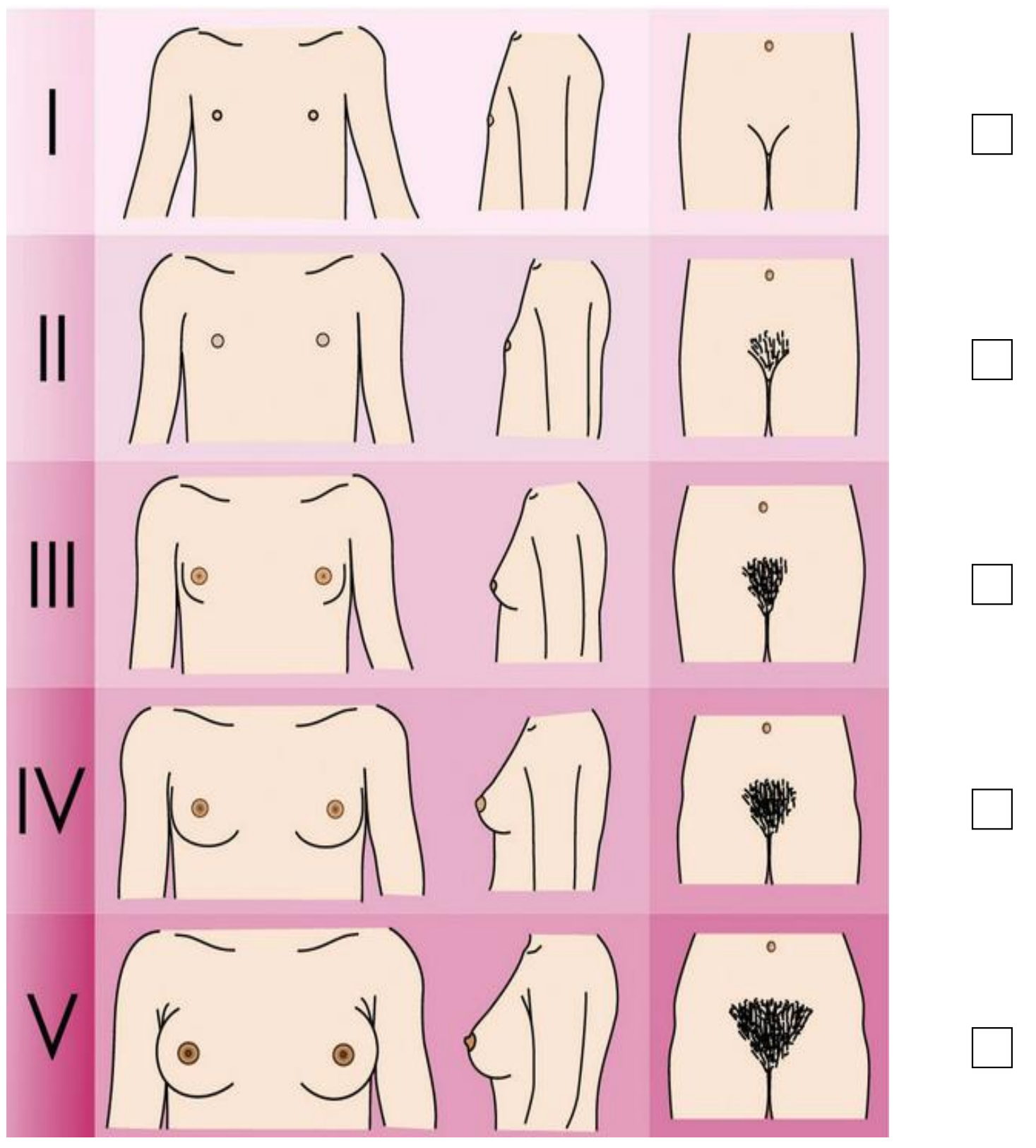


APPENDIX C:

\section{QUESTIONNAIRES}




\section{Appendix C: Questionnaires}

\section{Pediatric Quality of Life Inventory}

In the PAST MONTH, how much of a problem has this been for you ..

\begin{tabular}{|l|c|c|c|c|c|}
\hline About My Health AND ACtivitiEs (problems with...) & Never & $\begin{array}{c}\text { Ammost } \\
\text { Never }\end{array}$ & $\begin{array}{c}\text { Some- } \\
\text { times }\end{array}$ & Often & $\begin{array}{c}\text { Almost } \\
\text { Always }\end{array}$ \\
\hline $\begin{array}{l}\text { 1. It is hard for me to walk more than a couple of streets } \\
\text { (about 100 metres) }\end{array}$ & 0 & 1 & 2 & 3 & 4 \\
\hline 2. It is hard for me to run & 0 & 1 & 2 & 3 & 4 \\
\hline 3. It is hard for me to do sports activities or exercise & 0 & 1 & 2 & 3 & 4 \\
\hline 4. It is hard for me to lift heavy things & 0 & 1 & 2 & 3 & 4 \\
\hline 5. It is hard for me to have a bath or shower by myself & 0 & 1 & 2 & 3 & 4 \\
\hline 6. It is hard for me to do chores around the house & 0 & 1 & 2 & 3 & 4 \\
\hline 7. I have aches and pains & 0 & 1 & 2 & 3 & 4 \\
\hline 8. I feel tired & 0 & 1 & 2 & 3 & 4 \\
\hline
\end{tabular}

\begin{tabular}{|l|c|c|c|c|c|}
\hline ABout MY FEELINGs (problems with...) & Never & $\begin{array}{c}\text { Almost } \\
\text { Never }\end{array}$ & $\begin{array}{c}\text { Some } \\
\text { times }\end{array}$ & Often & $\begin{array}{c}\text { Almoet } \\
\text { Always }\end{array}$ \\
\hline 1. I feel afraid or scared & 0 & 1 & 2 & 3 & 4 \\
\hline 2. I feel sad & 0 & 1 & 2 & 3 & 4 \\
\hline 3. I feel angry & 0 & 1 & 2 & 3 & 4 \\
\hline 4. I have trouble sleeping & 0 & 1 & 2 & 3 & 4 \\
\hline 5. I worry about what will happen to me & 0 & 1 & 2 & 3 & 4 \\
\hline
\end{tabular}

\begin{tabular}{|l|c|c|c|c|c|}
\hline How I Get ON with OTHERs (problems with...) & Never & $\begin{array}{c}\text { Almost } \\
\text { Never }\end{array}$ & $\begin{array}{c}\text { Some- } \\
\text { times }\end{array}$ & Often & $\begin{array}{c}\text { Almost } \\
\text { Always }\end{array}$ \\
\hline 1. I have trouble getting on with other teenagers & 0 & 1 & 2 & 3 & 4 \\
\hline 2. Other teenagers do not want to be my friend & 0 & 1 & 2 & 3 & 4 \\
\hline 3. Other teenagers tease me & 0 & 1 & 2 & 3 & 4 \\
\hline 4. I cannot do things that other teenagers my age can do & 0 & 1 & 2 & 3 & 4 \\
\hline 5. It is hard to keep up with other teenagers my age & 0 & 1 & 2 & 3 & 4 \\
\hline
\end{tabular}

\begin{tabular}{|c|c|c|c|c|c|}
\hline ABOUt SCHOOL / COLLEGE (problems with...) & Never & $\begin{array}{l}\text { Almost } \\
\text { Never }\end{array}$ & $\begin{array}{l}\text { Some- } \\
\text { times }\end{array}$ & Often & $\begin{array}{l}\text { Almost } \\
\text { Always }\end{array}$ \\
\hline 1. It is hard to pay attention in class & 0 & 1 & 2 & 3 & 4 \\
\hline 2. I forget things & 0 & 1 & 2 & 3 & 4 \\
\hline $\begin{array}{l}\text { 3. I have trouble keeping up with my school / college } \\
\text { work }\end{array}$ & 0 & 1 & 2 & 3 & 4 \\
\hline 4. I miss school / college because of not feeling well & 0 & 1 & 2 & 3 & 4 \\
\hline 5. I miss school / college to go to the doctor or hospital & 0 & 1 & 2 & 3 & 4 \\
\hline
\end{tabular}


ASTHMA CONTROL QUESTIONNAIRE@ (ENGLISH VERSION FOR THE UK)
PATIENT ID:

DATE:

Page 1 of 2

Please answer questions $1-6$

Circle the number of the response that best describes how you have been during the past week.

1. On average, during the past week, how often were you woken by your asthma during the night?

$\begin{array}{ll}0 & \text { Never } \\ 1 & \text { Hardly ever } \\ 2 & \text { A few times } \\ 3 & \text { Several times } \\ 4 & \text { Many times } \\ 5 & \text { A great many times } \\ 6 & \text { Unable to sleep because of asthma }\end{array}$

3. In general, during the past week, how limited were you in your activities because of your asthma?

On average, during the past week, how bad were your asthma symptoms when you woke up in the morning?

No symptoms

1 Very mild symptoms

2 Mild symptoms

3 Moderate symptoms

4 Quite severe symptoms

5 Severe symptoms

6 Very severe symptoms

0 Not limited at all

1 Very slightly limited

2 Slightly limited

3 Moderately limited

4 Very limited

5 Extremely limited

6 Totally limited

4. In general, during the past week, how much shortness of breath did you experience because of your asthma?

0 None

1 A very little

2 A little

3 A moderate amount

4 Quite a lot

5 A great deal

6 A very great deal 
ASTHMA CONTROL QUESTIONNAIREC (ENGLISH VERSION FOR THE UK)
PATIENT ID:

DATE:

Page 2 of 2

5. In general, during the past week, how much of the time did you wheeze?
Never

Hardly any of the time

A little of the time

A moderate amount of the time

A lot of the time

Most of the time

All the time

6. On average, during the past week,

None

how many puffs/inhalations of short-acting 11 -2 puffs/inhalations most days

bronchodilator (eg. Ventolin/Bricanyl) have 23 -4 puffs/inhalations most days

you used each day?

5 - 8 puffs/inhalations most days

(If you are not sure how to answer this

9 - 12 puffs/inhalations most days

question, please ask for help)

13 - 16 puffs/inhalations most days

More than 16 puffs/inhalations most days

\section{To be completed by a member of the clinic staff}

7. FEV 1 pre-bronchodilator:

FEV 1 predicted:

FEV ${ }_{1} \%$ predicted:

(Record actual values on the dotted

lines and score the $\mathrm{FEV}_{1} \%$ predicted in the next column)
$0>95 \%$ predicted

$195-90 \%$

$289-80 \%$

$379-70 \%$

$4 \quad 69-60 \%$

$5 \quad 59-50 \%$

$6<50 \%$ predicted

Modified on 08 September 2010

ACQ - United Kingdom/English - Version of 08 Sep 10 - Mapi Research Institute 
PAEDIATRIC ASTHMA QUALITY OF LIFE QUESTIONNAIRE(S)

PATIENT ID

(ENGLISH VERSION FOR UNITED KINGDOM)

SELF-ADMINISTERED

DATE:

Page 1 of 4

Please complete all the questions by circling the number that best describes how you have been during the last week as a result of your asthma.

HOW BOTHERED HAVE YOU BEEN DURING THE LAST WEEK DOING:

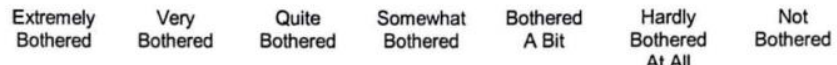

1. PHYSICAL ACTIVITIES

(such as running, swimming,

Bothered Bothered Bothered

At All

sports, walking uphill/upstairs

2

5

7

and bicycling)?

2. BEING WITH ANIMALS

(such as playing with pets

and looking after animals)?

2

3

4

5

6

7

3. ACTIVITIES WITH FRIENDS AND FAMILY (such as playing during school break and doing things with your friends and family)?

4. COUGHING

$\begin{array}{lllllll}1 & 2 & 3 & 4 & 5 & 6 & 7 \\ 1 & 2 & 3 & 4 & 5 & 6 & 7\end{array}$

IN GENERAL, HOW OFTEN DURING THE LAST WEEK DID YOU:

5. Feel FRUSTRATED because of your asthma? All of the Most of the Quite
Time Time Some of Once in a Hardly Any of the Tine of Feel TIRED because of

1
1

2

3

4

6 your asthma?

1

12

23

7. Feel WORRIED,

CONCERNED OR

TROUBLED because of your asthma? 
PAEDIATRIC ASTHMA QUALITY OF LIFE

QUESTIONNAIRE(S)

PATIENT ID

(ENGLISH VERSION FOR UNITED KINGDOM)

SELF-ADMINISTERED

DATE:

Page 2 of 4

HOW BOTHERED HAVE YOU BEEN DURING THE LAST WEEK BY?

\begin{tabular}{|c|c|c|c|c|c|c|}
\hline $\begin{array}{l}\text { Extremely } \\
\text { Bothered }\end{array}$ & $\begin{array}{c}\text { Very } \\
\text { Bothered }\end{array}$ & $\begin{array}{c}\text { Quite } \\
\text { Bothered }\end{array}$ & $\begin{array}{l}\text { Somewhat } \\
\text { Bothered }\end{array}$ & $\begin{array}{c}\text { Bothered } \\
\text { A Bit }\end{array}$ & $\begin{array}{l}\text { Hardly } \\
\text { Bothered } \\
\text { At All }\end{array}$ & $\begin{array}{c}\text { Not } \\
\text { Bothered }\end{array}$ \\
\hline
\end{tabular}

$\begin{array}{llllllll}\text { 8. ASTHMA ATTACKS } & 1 & 2 & 3 & 4 & 5 & 6 & 7\end{array}$

IN GENERAL, HOW OFTEN DURING THE LAST WEEK DID YOU:

$\begin{array}{llcccccc}\text { All of the } & \begin{array}{c}\text { Most of the } \\ \text { Time }\end{array} & \begin{array}{c}\text { Quite } \\ \text { Often }\end{array} & \begin{array}{c}\text { Some of } \\ \text { the Time }\end{array} & \begin{array}{c}\text { Once in a } \\ \text { While }\end{array} & \begin{array}{c}\text { Hardly Any } \\ \text { of the Time }\end{array} & \begin{array}{c}\text { None of the } \\ \text { Time }\end{array} \\ \text { 9. Feel ANGRY because of } & 1 & 2 & 3 & 4 & 5 & 6 & 7 \\ \text { your asthma? } & 1 & 2 & 3 & 4 & 5\end{array}$

HOW BOTHERED HAVE YOU BEEN DURING THE LAST WEEK BY?

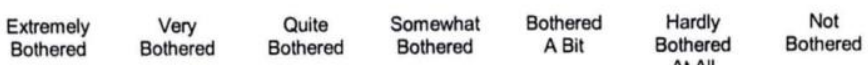

10. WHEEZING

23

4

5

6

7

IN GENERAL, HOW OFTEN DURING THE LAST WEEK DID YOU:

$\begin{array}{lccccccc} & \begin{array}{c}\text { All of the } \\ \text { Time }\end{array} & \begin{array}{c}\text { Most of the } \\ \text { Time }\end{array} & \text { Quite Often } & \begin{array}{c}\text { Some of } \\ \text { the Time }\end{array} & \begin{array}{c}\text { Once in a } \\ \text { While }\end{array} & \begin{array}{c}\text { Hardly Any } \\ \text { of the Time }\end{array} & \begin{array}{c}\text { None of the } \\ \text { Time }\end{array} \\ \begin{array}{l}\text { 1. Feel IRRITABLE } \\ \text { (grumpy) because of } \\ \text { your asthma? }\end{array} & 1 & 2 & 3 & 4 & 5 & 6 & 7 \\ \end{array}$

HOW BOTHERED HAVE YOU BEEN DURING THE LAST WEEK BY?

\begin{tabular}{|c|c|c|c|c|c|}
\hline $\begin{array}{l}\text { Extremely } \\
\text { Bothered }\end{array}$ & $\begin{array}{c}\text { Very } \\
\text { Bothered }\end{array}$ & $\begin{array}{c}\text { Quite } \\
\text { Bothered }\end{array}$ & $\begin{array}{c}\text { Somewhat } \\
\text { Bothered }\end{array}$ & $\begin{array}{c}\text { Bothered } \\
\text { A Bit }\end{array}$ & $\begin{array}{l}\text { Hardly } \\
\text { Bothered } \\
\text { At All }\end{array}$ \\
\hline
\end{tabular}

12. TIGHTNESS IN YOUR CHEST

3

4

5

6

7 
PAEDIATRIC ASTHMA QUALITY OF LIFE QUESTIONNAIRE(S)

(ENGLISH VERSION FOR UNITED KINGDOM)

SELF-ADMINISTERED

PATIENT ID:

DATE:

Page 3 of 4

IN GENERAL, HOW OFTEN DURING THE LAST WEEK DID YOU:

13. Feel DIFFERENT OR LEFT OUT because of your asthma?

All of the Most of the Quite Often Time $\quad$ Time

\section{DATE:}

HOW BOTHERED HAVE YOU BEEN DURING THE LAST WEEK BY?

\begin{tabular}{|c|c|c|c|c|c|c|c|}
\hline & $\begin{array}{l}\text { Extremely } \\
\text { Bothered }\end{array}$ & $\begin{array}{l}\text { Very } \\
\text { Bothered }\end{array}$ & $\begin{array}{l}\text { Quite } \\
\text { Bothered }\end{array}$ & $\begin{array}{l}\text { Somewhat } \\
\text { Bothered }\end{array}$ & $\begin{array}{l}\text { Bothered } \\
\text { A Bit }\end{array}$ & $\begin{array}{l}\text { Hardly } \\
\text { Bothered } \\
\text { At All }\end{array}$ & $\begin{array}{c}\text { Not } \\
\text { Bothered }\end{array}$ \\
\hline $\begin{array}{l}\text { 14. SHORTNESS OF } \\
\text { BREATH }\end{array}$ & 1 & 2 & 3 & 4 & 5 & 6 & 7 \\
\hline
\end{tabular}

IN GENERAL, HOW OFTEN DURING THE LAST WEEK DID YOU:

\begin{tabular}{|c|c|c|c|c|c|c|c|c|}
\hline 15. & $\begin{array}{l}\text { Feel FRUSTRATED } \\
\text { BECAUSE YOU } \\
\text { COULDN'T KEEP UP } \\
\text { WITH OTHERS? }\end{array}$ & 1 & 2 & 3 & 4 & 5 & 6 & 7 \\
\hline 16. & $\begin{array}{l}\text { WAKE UP DURING THE } \\
\text { NIGHT because of your } \\
\text { asthma? }\end{array}$ & 1 & 2 & 3 & 4 & 5 & 6 & 7 \\
\hline 17. & $\begin{array}{l}\text { Feel UNCOMFORTABLE } \\
\text { because of your asthma? }\end{array}$ & 1 & 2 & 3 & 4 & 5 & 6 & 7 \\
\hline 18. & $\begin{array}{l}\text { Feel OUT OF BREATH } \\
\text { because of your asthma? }\end{array}$ & 1 & 2 & 3 & 4 & 5 & 6 & 7 \\
\hline 19. & $\begin{array}{l}\text { Feel YOU COULDN'T } \\
\text { KEEP UP WITH } \\
\text { OTHERS because of } \\
\text { your asthma? }\end{array}$ & 1 & 2 & 3 & 4 & 5 & 6 & 7 \\
\hline 20. & $\begin{array}{l}\text { Have trouble SLEEPING } \\
\text { AT NIGHT because of } \\
\text { your asthma? }\end{array}$ & 1 & 2 & 3 & 4 & 5 & 6 & 7 \\
\hline 21. & $\begin{array}{l}\text { Feel FRIGHTENED BY } \\
\text { AN ASTHMA ATTACK? }\end{array}$ & 1 & 2 & 3 & 4 & 5 & 6 & 7 \\
\hline
\end{tabular}


PAEDIATRIC ASTHMA QUALITY OF LIFE

QUESTIONNAIRE(S)

PATIENT ID:

(ENGLISH VERSION FOR UNITED KINGDOM)

SELF-ADMINISTERED

DATE:

Page 4 of 4

THINK ABOUT ALL THE ACTIVITIES THAT YOU DID IN THE PAST WEEK:

\begin{tabular}{|c|c|c|c|c|c|}
\hline $\begin{array}{l}\text { Extremely. } \\
\text { Bothered }\end{array}$ & $\begin{array}{c}\text { Very } \\
\text { Bothered }\end{array}$ & $\begin{array}{c}\text { Quite } \\
\text { Bothered }\end{array}$ & $\begin{array}{l}\text { Somewhat } \\
\text { Bothered }\end{array}$ & $\begin{array}{c}\text { Bothered } \\
\text { A Bit }\end{array}$ & $\begin{array}{l}\text { Hardly } \\
\text { Bothered } \\
\text { At All }\end{array}$ \\
\hline
\end{tabular}

22. How much were you bothered by your asthm doing these activities?

$\begin{array}{lllllll}1 & 2 & 3 & 4 & 5 & 6 & 7\end{array}$

IN GENERAL, HOW OFTEN DURING THE LAST WEEK DID YOU:

\begin{tabular}{|c|c|c|c|c|}
\hline $\begin{array}{c}\text { All of } \\
\text { the Time }\end{array}$ & $\begin{array}{l}\text { Most of the } \\
\text { Time }\end{array}$ & $\begin{array}{l}\text { Some of } \\
\text { the Time }\end{array}$ & $\begin{array}{l}\text { Once in a } \\
\text { While }\end{array}$ & $\begin{array}{l}\text { Hardly Any } \\
\text { of the Time }\end{array}$ \\
\hline
\end{tabular}

23. Have difficulty taking a DEEP BREATH?

1

2

3

4

$5 \quad 6$

7

\section{DOMAIN CODE:}

Symptoms: 4, 6, 8, 10, 12, 14, 16, 18, 20, 23

Activity Limitation: 1, 2, 3, 19, 22

Emotional Function: 5, 7, 9, 11, 13, 15, 17, 21

Updated on 08 December 2010

PAOLQ(S)-SA - United Kingdom/English - Version of 08 Dec 10 - Mapi Research Institute.

ID5967/ PAQLQ(S)-SA_AU1.0 eng-GB.dOC 


\section{Healthcare Resource Questionnaire}

Name:

\section{HEALTH RESOURCE QUESTIONNAIRE}

1. In the last 3 months, how many times have you been to the nurse in your GP surgery for your asthma?

$\begin{array}{llllllllll}0 & 1 & 2 & 3 & 4 & 5 & 6 & 7 & 8 & 9 \text { or more }\end{array}$

2. How many of these were unplanned visits because your asthma was worse (not a routine check)?

$\begin{array}{llllllllll}0 & 1 & 2 & 3 & 4 & 5 & 6 & 7 & 8 & 9 \text { or more }\end{array}$

3. In the last 3 months, how many times have you been to the doctor in your GP surgery for your asthma?

$\begin{array}{llllllllll}0 & 1 & 2 & 3 & 4 & 5 & 6 & 7 & 8 & 9 \text { or more }\end{array}$

4. How many of these were unplanned visits because your asthma was worse (not a routine check)?

$\begin{array}{llllllllll}0 & 1 & 2 & 3 & 4 & 5 & 6 & 7 & 8 & 9 \text { or more }\end{array}$

5. In the last 3 months, how many times have you been to A\&E for your asthma?

$\begin{array}{llllllllll}0 & 1 & 2 & 3 & 4 & 5 & 6 & 7 & 8 & 9 \text { or more }\end{array}$

6. In the last 3 months, how many nights have you stayed in hospital for your asthma?

$\begin{array}{llllllllll}0 & 1 & 2 & 3 & 4 & 5 & 6 & 7 & 8 & 9 \text { or more }\end{array}$

7. In the last 3 months, how many times have you been to the hospital clinic for your asthma?

$\begin{array}{llllllllll}0 & 1 & 2 & 3 & 4 & 5 & 6 & 7 & 8 & 9 \text { or more }\end{array}$ 
Name:

8. In the last 3 months, how many days of school have you missed because of your asthma?

9. What is the name of your reliever inhaler? (e.g. Ventolin). If you don't know the name, just write the colour (e.g. blue). (if not on a reliever inhaler please write 'Nil')

10. What is the dose of your reliever inhaler? $(100,500$, Don't know, Other [If other state what does; if not on a reliever inahaler please write 'Nil'])

If other - state what dose

11. Write how many puffs of your reliever do you take every day/week/month? (e.g. 2 puffs a day, 4 puffs a week, 4 puffs a month; if not on a reliever inhaler please write 'Nil')

12. What is the name of your preventer inhaler? (e.g. Becotide, Flixotide). If you don't know the name, just write the colour (e.g. brown, orange, purple); If not on a preventer, write 'Nil'.

13. What is the dose of your preventer inhaler? $(50,100,200,250$, Don't know, Other [if other - state what does] if not on a preventer, write 'Nil')

14. How many puffs of your preventer do you take every day? (e.g. 2 puffs twice a day; if not on a preventer, write 'Nil')

15. If you are on any other treatment for asthma then write it here. (e.g Singulair/Montelukast 10mg (or write 'one tablet') once a day; if not on any other treatment please write 'Nil') 
Name:

16. In the last 3 months, how many courses of steroid tablets have you taken for asthma?

$\begin{array}{llllllllll}0 & 1 & 2 & 3 & 4 & 5 & 6 & 7 & 8 & 9 \text { or more }\end{array}$

17. In the last 3 months, how many courses of antibiotic tablets have you taken for asthma?

$\begin{array}{llllllllll}0 & 1 & 2 & 3 & 4 & 5 & 6 & 7 & 8 & 9 \text { or more }\end{array}$

18. What was the name(s) of the antibiotic(s), if none please write Nil? 


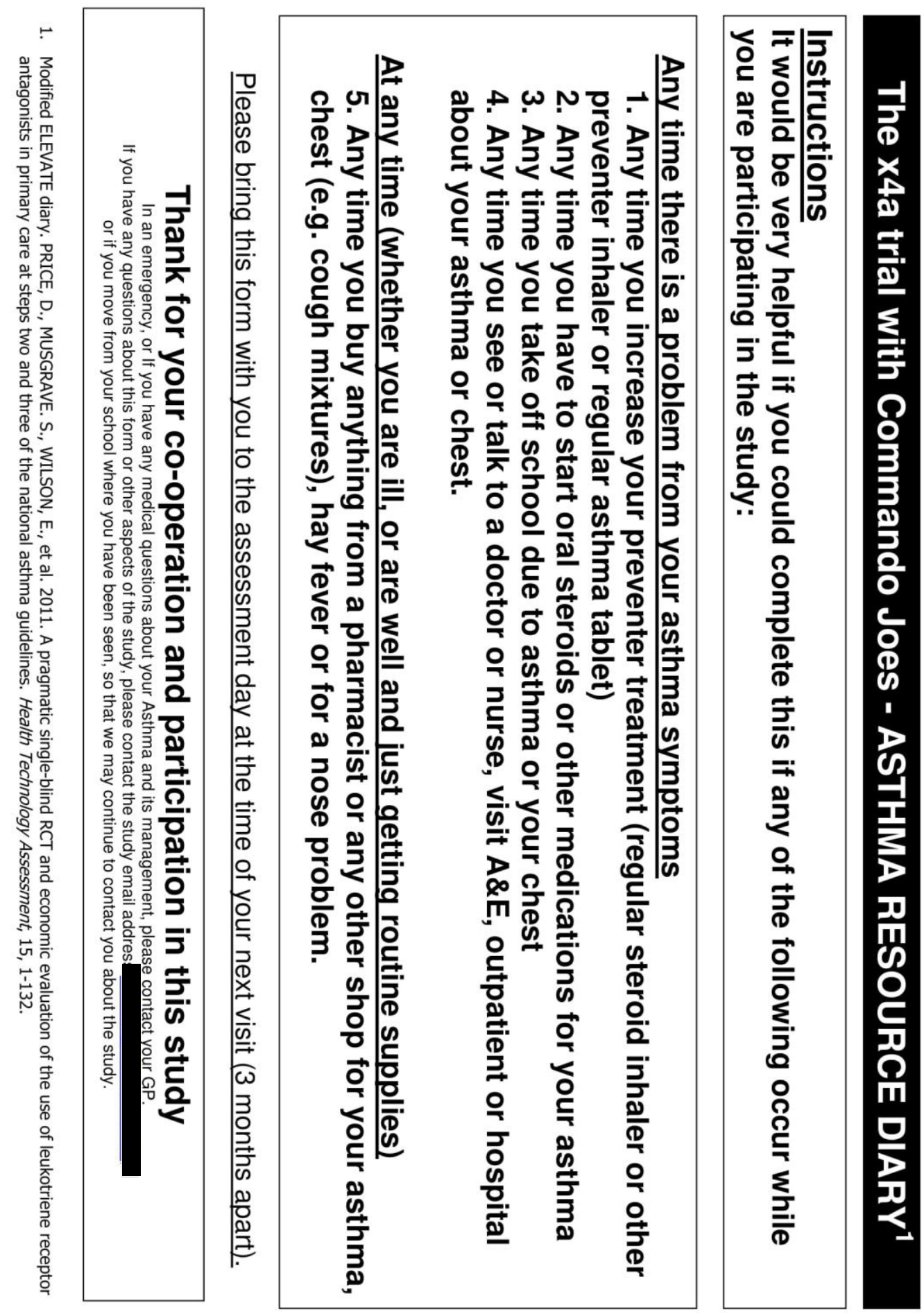




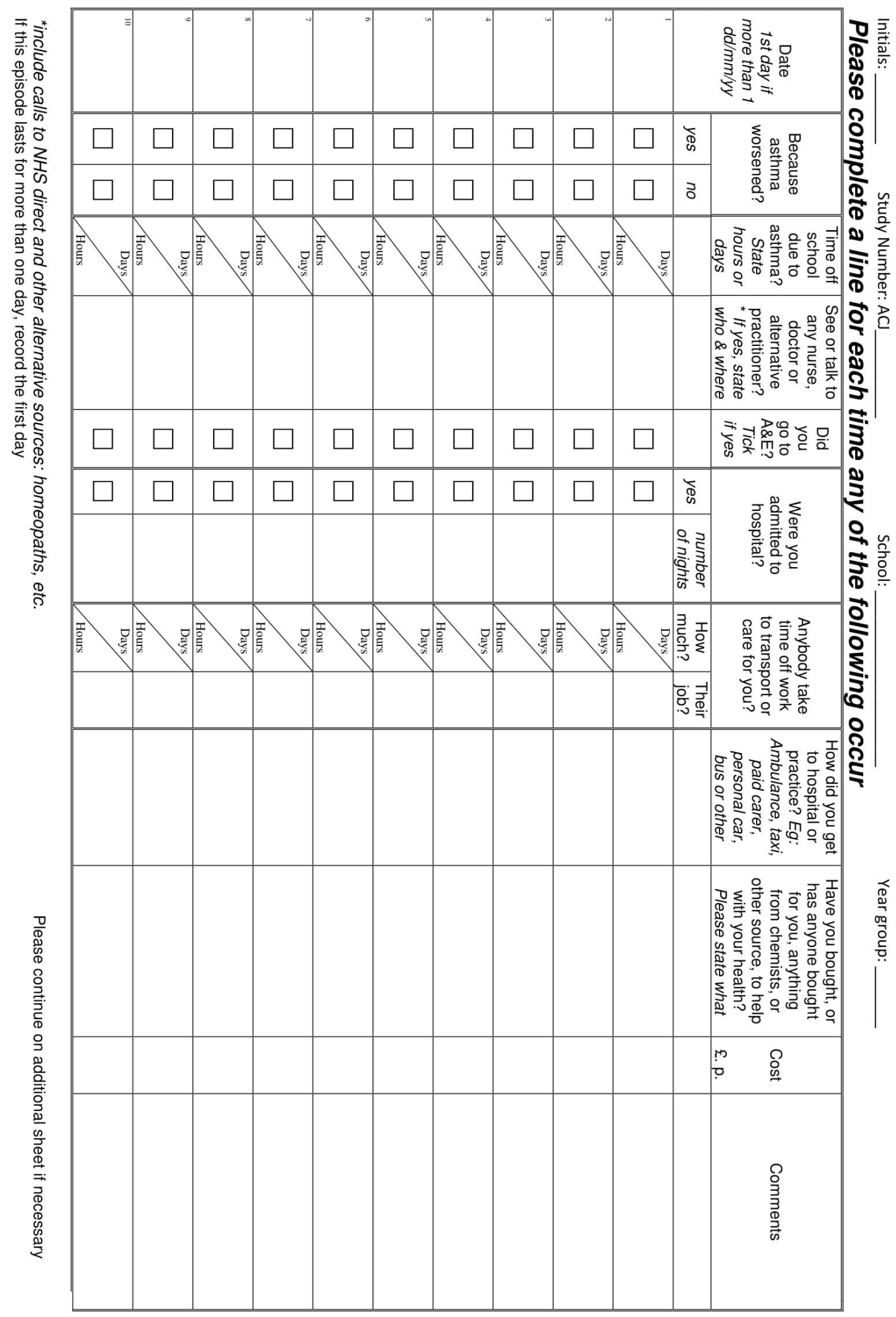


